\title{
X-Ray Beam Studies of Charge Sharing in Small Pixel, Spectroscopic, CdZnTe Detectors
}

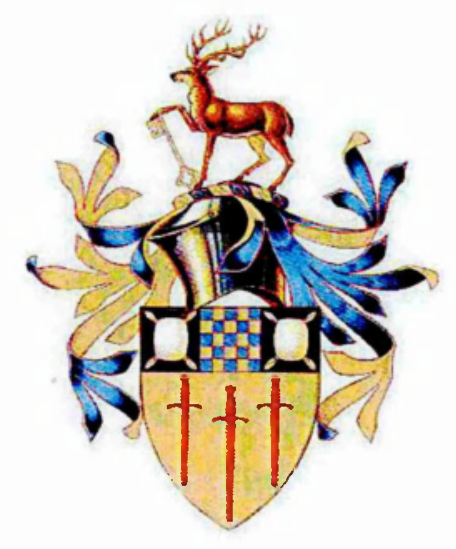

Christopher Allwork

Submitted for the degree of Doctor of Philosophy

Department of Physics,

University of Surrey,

Guildford,

GU2 7XH

February 7, 2013 
ProQuest Number: 27558334

All rights reserved

INFORMATION TO ALL USERS

The quality of this reproduction is dependent upon the quality of the copy submitted.

In the unlikely event that the author did not send a complete manuscript and there are missing pages, these will be noted. Also, if material had to be removed, a note will indicate the deletion.

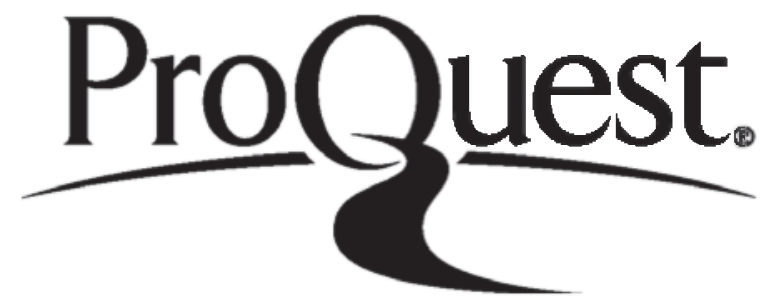

ProQuest 27558334

Published by ProQuest LLC (2019). Copyright of the Dissertation is held by the Author.

All rights reserved.

This work is protected against unauthorized copying under Title 17, United States Code Microform Edition (C) ProQuest LLC.

ProQuest LLC.

789 East Eisenhower Parkway

P.O. Box 1346

Ann Arbor, Ml $48106-1346$ 
Dedicated to Mandy the best mother you could ask for. Would never of got here without you... 


\section{Abstract}

CdZnTe has been the focus of intense research as an attractive alternative material as a semiconductor radiation detector. Due to recent advances in the growth of the CdZnTe crystals it has been possible to develop small pixel, spectroscopic radiation detectors which overcome the poor hole transport properties of CdZnTe. This project utilized the PIXIE ASIC to investigate effects relating to pixelated detectors. This work focused a wide range of effects relating to pixelated CdZnTe radiation detectors. Investigations into single pixel effects resulted in investigation how different components of electronic noise effect detector performance. Furthermore the charge collection efficiency of each pixel was investigated to quantify its effect on the spectroscopic performance. Multiple pixel effects, for example charge sharing between adjacent pixels when pixel size is significantly smaller that detector thickness was investigated. X-ray spectroscopy studies were undertaken to investigate how the level of charge sharing is effected by the incident X-ray energy and the pixel size of the pixel array. Charge sharing and charge loss in a $250 \mu \mathrm{m}$ pitch $\mathrm{CdZnTe}$ pixel detector was also investigated using a mono-chromatic X-ray beam at the Diamond Light Source, U.K. Using a $20 \mu \mathrm{m}$ beam diameter the detector response has been mapped for X-ray energies both above (40 keV) and below (26 $\mathrm{keV}$ ) the material $K$-shell absorption energies to study charge sharing and the role of fluorescence X-rays in these events. Furthermore how the bias applied to detector effects the level of charge sharing and charge loss will be investigated. 


\section{Acknowledgements}

Firstly I would like to thank my supervisor Paul Sellin. Who has always been considerate to the difficult circumstances over the last three years and has been source of help and advise throughout.

Many thanks should also go to the $\mathrm{PhD}$ students, postdocs and academics in the radiation detection group. Annika Lohstroh, Dimitirs Kitou, Veeramani Permual, Paola Avella and Sandeep Chaudhuri whose help and support throughout the three years was invaluable. I would also like to thank Cristobel Soares-Smith for always being available for a chat and for her unwavering help in setting up meetings with Paul. Another special thanks should also go to all my fellow wreckers. The last seven years at Surrey have been most fun and rewarding experiences I could have ever wished for and that's because of you guys and girls.

I would also like to thank Nicola Tartoni at the Diamond Light Source for being so generous in providing the 2 days of beam time which were so crucial for this project. A big thanks should also go to Matt Veale at the Rutherford Appleton Laboratories (RAL) whose help and advise over the last three years has been invaluable. Furthermore thanks should go to John Lipp at RAL for his help in the final year of this project.

On a personal note I would like to thank all the staff at the Royal Surrey County Hospital for all there help with my back problems during these last three years. I would like to thank all my family who have been so supportive through the most difficult times of all our lives.

Finally the most important acknowledgement should go to my mother Mandy who is no longer with us. Her support and constant source of love through my whole live resulted in all my achievements to date. She did such a great job of raising myself and my sister and all our future successes will be rooted in her greatness as a mother and a friend. 


\section{Contents}

Abstract i

$\begin{array}{ll}\text { Acknowledgements } & \text { ii }\end{array}$

1 Introduction $\quad 1$

1.1 Semiconductor Radiation detectors . . . . . . . . . . . . . 2

1.2 Cadmium Zinc Telluride $(\mathrm{CdZnTe}) \ldots \ldots \ldots$

1.2.1 Pixelated CdZnTe Radiation Detectors . . . . . . . . . . 5

1.3 Thesis Outline . . . . . . . . . . . . . . 7

2 Detector Physics Theory 9

2.1 Interaction of Electromagnetic Radiation With Matter . . . . . . 9

2.1.1 Photoelectric Absorption . . . . . . . . . . . . . 10

2.1.2 Compton Scattering . . . . . . . . . . . . 11

2.1 .3 Pair Production . . . . . . . . . . . . . . . . . . . 12

2.1.4 X-ray and $\gamma$-ray Attenuation Coefficient . . . . . . . . 13

2.2 X-ray Fluorescence . . . . . . . . . . . . . . . . . . . 14

2.3 Semiconductor Band Structure . . . . . . . . . . . . . . 17

2.4 Generation of Charge Carriers . . . . . . . . . . . . . . 21

2.5 The Shockley-Ramo Theorem . . . . . . . . . . . . . . . 22

2.6 Single Charge Carrier Sensitive Detectors . . . . . . . . . . . . 25

2.6.1 Pixelated CdZnTe Radiation Detectors . . . . . . . . . . 27

2.6.2 Charge Sharing Between Adjacent Pixels . . . . . . . . . . . 29 
3 Experimental Setup and Apparatus 33

3.1 Fabrication of $\mathrm{CdZnTe}$ Detectors . . . . . . . . . . . 33

3.2 Surface Characterisation Methods . . . . . . . . . . . . . 36

3.2 .1 Zygo Newview $200 \ldots \ldots$. . . . . . . . . . . 36

3.2 .2 Scanning Electron Microscope (SEM) . . . . . . . . . . 37

3.3 PIXIE ASIC and Readout System . . . . . . . . . . . . . 37

3.4 XIA DGF-Pixie-4: Digital Data Acquisition System . . . . . . . . . 41

3.5 LabVIEW Digital Pulse Processing Analysis Software . . . . . . . 42

3.5.1 CR- $(\mathrm{RC})^{n}$ Digital Pulse Shaping . . . . . . . . . . 43

3.5.2 Pixel Spectra Generation . . . . . . . . . . . . . 47

3.5.3 Event Classification and Sum Spectrum Generation . . . . . 47

3.6 Focused X-ray Beamline Studies . . . . . . . . . . . . 50

4 CdZnTe Detector Metrology and Spectroscopy $\mathbf{5 3}$

4.1 Metrology Studies of a CdZnTe Medipix Detector . . . . . . . . . 54

4.1 .1 Curvature of CdZnTe material . . . . . . . . . . . . 54

4.1.2 Surface Roughness of the Bare CdZnTe Material . . . . . . . 57

4.1.3 Gold Pixel Characterisation . . . . . . . . . . . . . . 58

4.2 Spectroscopy Studies of CdZnTe Redlen PIXIE Detector . . . . . . 61

4.2 .1 Single Pixel Spectra . . . . . . . . . . . . 6 61

4.2.1.1 Optimisation of LabVIEW Software Parameters . . 63

4.2.1.2 Detector Bias Effect on Detector Performance . . . . 68

4.2 .2 Sum Spectra and Multiple Pixel Effects . . . . . . . . . . 72

4.2.2.1 Gain Matching of the Detector . . . . . . . 72

4.2.2.2 Event Classification and the Optimum Threshold Value 78

4.2.2.3 Charge Sharing and Sum Spectrum Performance . . 80

4.3 Summary . . . . . . . . . . . . . . . . . . 84

5 Focused X-ray Beam Studies $\quad 87$

5.1 Array and Pixel Locating . . . . . . . . . . . . . . . . . . 88

5.2 Single Pixel Spectra . . . . . . . . . . . . . . . . . 92 
5.3 Variation in Charge Collected by a Single Pixel . . . . . . . . . . . 95

5.4 Charge Loss in the Interpixel Region . . . . . . . . . . . . . 96

5.5 2D Parametric Plot: Representation of Charge Sharing Events . . . 101

5.5 .1 2D plots Recorded with a $26 \mathrm{keV}$ Beam . . . . . . . . . 102

5.5.2 2D plots Recorded with a $40 \mathrm{keV}$ Beam . . . . . . . . . 105

5.6 Charge Sharing Between Adjacent Pixels at Different X-ray Energies 110

5.7 Detector Bias effect on Detector Characteristics . . . . . . . . . . 112

5.7 .1 Charge Sharing at Different Detector Biases ... . . . . 113

5.7.2 Variation in Total Collected Charge at Different Detector Biases114

5.8 Summary . . . . . . . . . . . . . . . . . . . 117

6 Conclusions and Future Work 120

6.1 Conclusions . . . . . . . . . . . . . . . . 120

6.2 . Future Work . . . . . . . . . . . . . . . . . 123

$\begin{array}{lr}\text { A Published Papers } & \mathbf{1 2 5}\end{array}$

$\begin{array}{lr}\text { List of Figures } & 150\end{array}$

List of Tables 


\section{Chapter 1}

\section{Introduction}

Since the discovery of radioactive materials, scientists have endeavoured to find efficient systems to detect and measure these various forms of radiation. Multiple systems have been developed to measure radiation and can be grouped into three different groups: gas filled detectors, scintillation detectors and semiconductor detectors.

The most common and widely used example of a gas filled radiation detector is a Geiger-Müller (G.M) Tube. Introduced by Hans Geiger and Walther Müller in 1928 a G.M tube can be used to detect ionising radiation such as alpha and beta particles. The detector consists of a tube of inert gas such as neon or argon between two electric plates. The gas is held in a high electric field so when radiation enters the tube the gas becomes ionized and avalanche occurs resulting in a high measurable current. The number of ions created is independent of the radiation energy therefore no information on the indecent energy of the radiation can be gained, however G.M. tubes are still widely used in the radiation monitoring sector.

In the early 1950's scintillation detector systems had been developed which made it possible to detect and measure the energy of radiation entering the detector. These detector systems consist of scintillation crystal coupled to a photomultiplier tube. When radiation enters the scintillator the atoms are placed in an excited state which then de-excite via the emission of photons with wavelengths in the visible to near-visible electromagnetic spectrum region. These photons then enter 
a photomultiplier tube which consists of a photocathode and a series of dynodes. The photon enters the photocathode and via the photoelectric effect a number of photoelectrons are emitted which are then multiplied in number by a series of dynode plates. This process results in a detectable signal which is proportional to the energy of the incoming radiation. By using different scintillation crystals it is possible to measure different types of radiation. For example NaI(Tl) scintillation detectors are optimized for measuring gamma radiation compared to CsI which is optimized for the detection of protons and alpha particles. The use of scintillation detectors have many desirable properties, however there are a significant number of steps to create a usable signal of which some can be inefficient resulting in degradation in the energy resolution. This meant much research was undertaken on detector systems which are more efficient for example the use of semiconductor materials.

\subsection{Semiconductor Radiation detectors}

Semiconductors have certain properties which make them a desirable alternative radiation detector compared to gas filled detectors and scintillation detectors. A detector has to be able to work under a high bias whilst still not creating a high leakage current which is a property of an insulator, however when charge carriers are created by the interaction of radiation with the detector material they have to be able to migrate through the detector hence act as a conductor. These two required properties meant much research was undertaken on semiconductor materials as possible candidates for radiation detectors.

When radiation enters the semiconductor electron-hole pairs are created, where the number of them is proportional to the energy of the incident radiation. The migration of these charge carriers can be converted to a readable signal which can then be used to measure the energy of the radiation. One desirable property of semiconductors compared to scintillators is the energy required to create an electronhole pair ( $W$-value). For example the $W$-value of $\mathrm{CdZnTe}$ is $\sim 5 \mathrm{eV}$ compared to $25 \mathrm{eV}$ for $\mathrm{NaI}(\mathrm{Tl})$ scintillator detector [1]. This decrease in $W$-value for CdZnTe 
means more charge carriers are created for the interaction of radiation with the same energy. As a result there is a larger signal-to-noise ratio and a better energy resolution.

The most widely used semiconductors for the detection of radiation are Silicon ( $\mathrm{Si}$ ) and Germanium (Ge). Both these materials give extremely high resolution and through years of research are easier to produce. However they do have certain disadvantages.

\begin{tabular}{|c|c|c|c|c|}
\hline Material & $\mathrm{Cd}_{0.9} \mathrm{Zn}_{0.1} \mathrm{Te}$ & $\mathrm{CdTe}$ & $\mathrm{Ge}$ & $\mathrm{Si}$ \\
\hline Atomic Number & 49.1 & 50 & 32 & 14 \\
\hline Density $\left(\mathrm{gcm}^{-3}\right)$ & 5.78 & 5.85 & 5.33 & 2.33 \\
\hline Band Gap $(\mathrm{eV})$ & 1.57 & 1.51 & 0.67 & 1.12 \\
\hline$W$-Value $(\mathrm{eV})$ & 4.6 & 4.4 & 3.0 & 3.6 \\
\hline Resistivity $\left(\Omega \mathrm{cm}^{2}\right.$ & $3 \times 10^{10}$ & $10^{9}$ & 50 & $<10^{4}$ \\
\hline Electron Mobility $\mu_{e}\left(\mathrm{~cm}^{2} \mathrm{~V}^{-1} \mathrm{~s}^{-1}\right)$ & 1000 & 1100 & 3900 & 1400 \\
\hline Electron Lifetime $\tau_{e}(\mathrm{~s})$ & $3 \times 10^{-6}$ & $3 \times 10^{-6}$ & $10^{-3}$ & $10^{-3}$ \\
\hline Hole Mobility $\mu_{h}\left(\mathrm{~cm}^{2} \mathrm{~V}^{-1} \mathrm{~s}^{-1}\right)$ & $10-80$ & 100 & 1900 & 480 \\
\hline Hole Lifetime $\tau_{h}(\mathrm{~s})$ & $1 \times 10^{-6}$ & $2 \times 10^{-6}$ & $1 \times 10^{-3}$ & $2 \times 10^{-3}$ \\
\hline
\end{tabular}

Table 1.1: Detector material properties of different semiconductor radiation detectors [2].

As shown in table 1.1 the band gap of $\mathrm{Si}$ and $\mathrm{Ge}(1.12 \mathrm{eV}$ and $0.67 \mathrm{eV}$ respectively) are lower compared to CdTe and $\mathrm{Cd}_{0.9} \mathrm{Zn}_{0.1} \mathrm{Te}$. The smaller band gap energy means the $W$-value is lower resulting in more charge carriers being created for the same incident radiation energy compared to $\mathrm{CdTe}$ and $\mathrm{Cd}_{0.9} \mathrm{Zn}_{0.1} \mathrm{Te}$. However the lower band gap does have one disadvantage. Equation 1.1 is the probability for carriers to be thermally generated $(p(T))$ where $T$ is the absolute temperature, $C$ is a material dependent constant, $k_{B}$ is Boltzmann's constant and $E_{g}$ is the band gap energy [3].

$$
p(T)=C T^{3 / 2} \exp \left(\frac{-E_{g}}{2 k_{B} T}\right)
$$

The lower band gap energy of $\mathrm{Si}$ and Ge will result in more thermally generated carriers thus increasing the measured leakage current of the detector. This increased leakage current will result in a degradation of the detector performance and for $\mathrm{Ge}$ detectors the detector needs to be cooled to cryogenic temperatures with systems 
which can be bulky and impractical for industrial applications.

Another disadvantage of Silicon and Germanium is the low atomic numbers $(Z)$ of the elements. Photoelectric absorption is the favoured gamma-ray interaction for a radiation detector where the probability for the interaction to occur is highly dependent on the $Z$ number of the material. As shown in figure 1.1 at higher energies the probability for photoelectric absorption as a function of energy is larger for CdZnTe compared to Si and Ge.

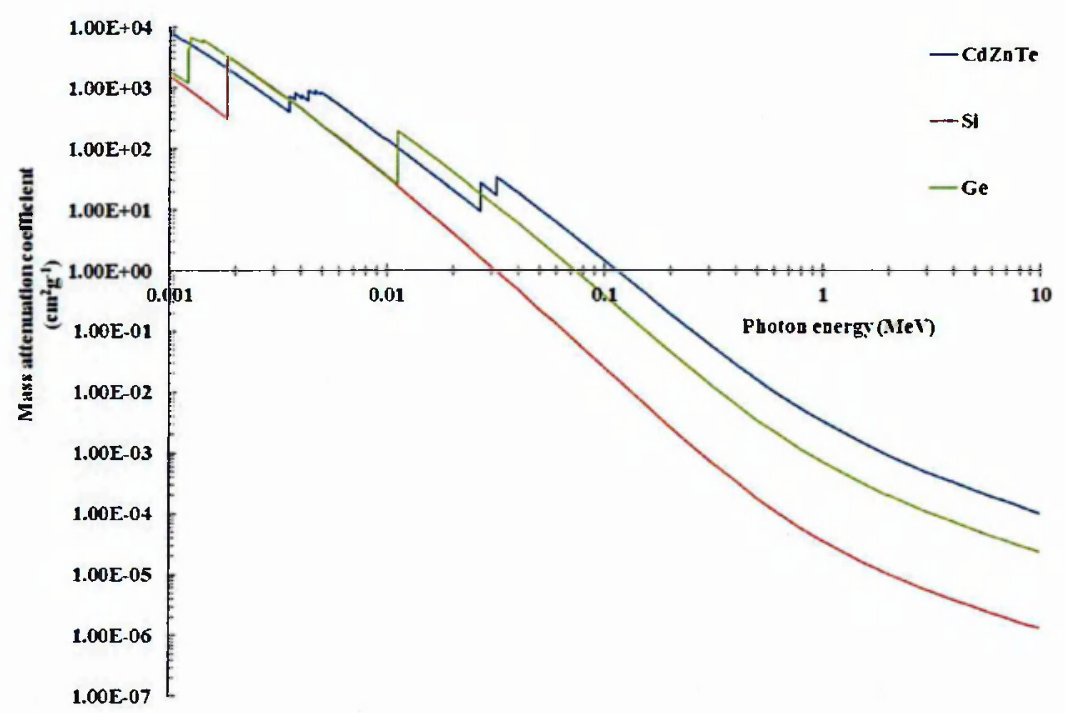

Figure 1.1: Probaility for photoelectric absorption to occur as function of energy for $\mathrm{Cd}_{0.9} \mathrm{Zn}_{0.1} \mathrm{Te}$, Ge and Si calculated using XCOM [4].

As a result of these disadvantages much research has been undertaken to find alternative semiconductors as potential candidates for radiation detectors. Compound semiconductors such as Gallium Arsenide (GaAs), Aluminium Gallium Arsenide (AlGaAs), Cadmium Telluride (CdTe), Cadmium Zinc Telluride (CdZnTe) and Mercuric Iodide $\left(\mathrm{HgI}_{2}\right)$ have been investigated as alternatives. These materials can be tuned to cater for industrial applications by changing the elements within the compound resulting in different band gaps and photoelectric coefficients. Much scientific research has been carricd out on CdZnTe as an alternative semiconductor radiation detector material for a wide variety of applications and has been investigated in this project. 


\subsection{Cadmium Zinc Telluride (CdZnTe)}

Cadmium Telluride (CdTe) is a II/VI binary compound semiconductor. Its higher band gap makes it possible to work reliably at room temperature which is desirable for applications in the medical, security and industrial sectors [5-9]. Furthermore the higher density and average atomic number compared to $\mathrm{Si}$ and Ge makes it a desirable alternative semiconductor. It is also possible to improve the properties of CdTe by replacing a percentage $(x)$ of the Cd atoms in the crystal lattice with $\mathrm{Zn}$ resulting in $\mathrm{Cd}_{1-x} \mathrm{Zn}_{x} \mathrm{Te}$ [10]. When $10 \%$ of the $\mathrm{Cd}$ atoms are replaced with $\mathrm{Zn}$ the band gap of the material increases from $1.51 \mathrm{eV}$ to $1.57 \mathrm{eV}$. This larger band gap and reasonable electron charge carrier transport properties meant much research was undertaken on $\mathrm{Cd}_{1-x} \mathrm{Zn}_{x} \mathrm{Te}$.

$\mathrm{Cd}_{1-x} \mathrm{Zn}_{x}$ Te has one significant limiting factor on its performance as a radiation detector. The charge transport properties of holes in CdZnTe is significantly lower compared to electrons $\left((\mu \tau)_{e}=3 \times 10^{-3} \mathrm{~cm}^{2} \mathrm{~s}^{-1},(\mu \tau)_{h}=5 \times 10^{-5} \mathrm{~cm}^{2} \mathrm{~s}^{-1}\right)$ [2]. As a consequence of this the generated holes are trapped in the crystal and it is thought to be due to defects and impurities entering the crystal in the growing procedure. The trapping of holes results in low energy tailing on a photopeak and a drop in detector performance. To account for this much research has been undertaken on developing detectors which are only sensitive to the movement of electrons thus negating the poor hole transport properties. One such example is the use of a pixelated anode and planar cathode.

\subsubsection{Pixelated CdZnTe Radiation Detectors}

The use of a detector with a pixelated anode and planar cathode results in the 'small pixel effect'. In these detectors the resulting signal generated by the movement of charge is dominated by the electron motion $[11,12]$. The 'small pixel effect' where the pixel size is significantly smaller than detector thickness can be utilized in pixel detectors. As the pixel size decreases compared to the detector thickness the detector sensitivity to hole motion is minimized resulting in an improved spectroscopic 
performance of the CdZnTe pixel detector [13]. A great deal on scientific research has been undertaken on pixelated $\mathrm{Cd}_{1-x} \mathrm{Zn}_{x}$ Te radiation detectors for a wide variety of applications in the medical, security, astrophysical and industrial sectors [5-9]. The choice of pixel size has a large effect on the recorded performance of pixelated radiation detectors [14]. For example in medical imaging applications there is need for a high level of positional resolution. This as a result means for medical imaging applications the use of small pixel sizes and pitches is a desirable property. However as the pixel size reduces compared to the detector thickness it is possible for charge to be shared between adjacent pixels and be lost in the interpixel region. In extreme cases where there is a high level of charge sharing the events may have to be reconstructed $[15,16]$. Charge sharing and charge loss in pixelated detectors can therefore have a large effect on the detector characteristics and performance.

The PIXIE ASIC has been developed by the Rutherford Appleton Laboratories to investigate a wide variety of effects relating to pixelated radiation detectors $[17,18]$. A PIXIE detector consists of four $3 \times 3$ pixel arrays of different pixel sizes, pitches and geometries. This project involved an extensive study of a Redlen CdZnTe PIXIE detector. Investigations into the spectroscopic performance of the detector and charge sharing between pixels was undertaken. Furthermore the use of a synchrotron X-ray beam at the Diamond Light Source made it possible to undertake studies into how the level of charge sharing and charge loss changes with incident radiation energies below (26 keV) and above $(40 \mathrm{keV})$ the $K$-shell absorption energy of $\mathrm{Cd}$ $(26.7 \mathrm{keV})$ and $\mathrm{Te}(31.8 \mathrm{keV})$.

Another example of a pixelated radiation detector is a Medipix detector. A Medipix detector [9] can be used in a wide variety of imaging applications due to its small pixel pitch size. A metrology study of a Medipix CdZnTe detector was undertaken to investigate it's suitability to be Indium (In) bump bonded to a Medipix III ASIC. 


\subsection{Thesis Outline}

The use of pixelated anode electrode as a way to improve the recorded performance of a CdZnTe radiation detector has been well known in recent years. This project involved significant studies into phenomena involved with pixelated radiation detectors. Charge sharing, charge loss and metrology studies of 2 different pixelated detectors were undertaken and will be explained in this thesis. What follows is a brief outline of each of the chapters in this thesis following this introduction chapter.

\section{- Chapter 2}

Chapter 2 involves discussions into the relevant detector theory for the operation of semiconductor radiation detectors such as CdZnTe. The chapter will explain how electromagnetic radiation interacts with material. Following on from this discussions into semiconductor theory and how charge carriers are generated inside the crystal. Furthermore explanations of the ShockleyRamo theorem regarding charge induction and single charge carrier sensitive detectors will be undertaken.

- Chapter 3

Chapter 3 focuses on the different experimental apparatus and setups used in this project. Initially a brief explanation of how gold pixel contacts are fabricated onto $\mathrm{CdZnTe}$ will be undertaken. Following on from this the section explains three different topics. Firstly the Zygo Newview 200 non-contact optical profiler and the function of a scanning electron microscope will be explained in relation to the use of the equipment in the metrology study of a Medipix CdZnTe detector. Secondly the PIXIE ASIC, the digital data acquisition system and the LabVIEW software written to analyse the data generated by the PIXIE ASIC in conjunction with the acquisition system will be explained. Finally the experimental setup used on the I15 beamline at Diamond Light Source to investigate charge sharing between pixels will be discussed. 
- Chapter 4

Chapter 4 is the first results chapter. Firstly the findings of a metrology study on a Medipix CdZnTe detector and its suitability to be In bump bonded to a Medipix III will be discussed. Following on from this an extensive study of a $2 \mathrm{~mm}$ thick Redlen CdZnTe PIXIE will be explained. Studies into single and multiple pixel effects in pixelated detectors and the dependence on incident X-ray energy and detector bias will be reviewed.

\section{- Chapter 5}

Chapter 5 focuses on an experimental campaign carried on the $\mathrm{I} 15$ beamline at the Diamond Light Source. Initially the procedure used to find the pixels of interest on the Redlen PIXIE detector characterized in chapter 4 will be explained. Following on from this the results gained when line scans between adjacent pixels were performed with a $20 \mu \mathrm{m}$ focused X-ray beam will analysed. Studies into how charge sharing and charge loss varies with incident X-ray energies below $(26 \mathrm{keV})$ and above $(40 \mathrm{keV})$ the mean $K$-shell absorption energies of $\mathrm{Cd}$ and $\mathrm{Te}$ will be discussed. Finally how the level of charge sharing and charge loss is effected by the detector bias will be discussed.

- Chapter 6

Chapter 6 will conclude the thesis which will summarise the major findings and discuss any suggestions for future work. 


\section{Chapter 2}

\section{Detector Physics Theory}

To understand the properties of the CdZnTe radiation detectors discussed in this thesis a good understanding of how a detectable signal is generated is essential. In this chapter topics regarding the interaction of ionising radiation with the detector material, the generation of charge carriers and the subsequent induced charge on an electrode will be discussed. Furthermore the concept of single charge carrier sensitive devices and the subsequent improved performance of a CdZnTe detector will be discussed.

\subsection{Interaction of Electromagnetic Radiation With Matter}

When radiation enters an absorbing material it can deposit a proportion, if not all of its initial energy via the interaction of the radiation with the atoms that make up the material. For the case of electromagnetic radiation like $\mathrm{X}$ rays and $\gamma$ rays there are three dominant interaction methods which are: photoelectric absorption, Compton scattering and pair production. As shown in figure 2.1 the probability for interaction to occur is dependant on the atomic number $(Z)$ of the material and the initial energy of the incoming radiation. 


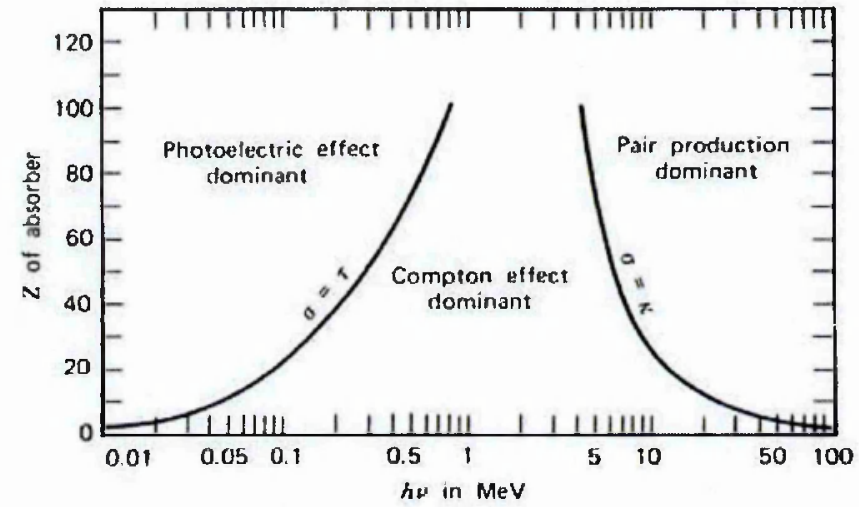

Figure 2.1: Graph to show how the relative importance of the three major X-ray and $\gamma$-ray interactions vary with $Z$ of absorber and the energy of the interacting radiation $[3,19]$.

\subsubsection{Photoelectric Absorption}

For a spectroscopic radiation detector such as $\mathrm{CdZnTe}$, photoelectric absorption is the most important interaction mechanism. This is due to the fact that when electromagnetic radiation interacts with the detector via photoelectric absorption it can deposit up to all of its energy.

As shown in figure 2.1 at energies less than $\sim 100 \mathrm{keV}$ photoelectric absorption is the dominant interaction mechanism. In photoelectric absorption the photon energy is transferred to a bound electron typically in the tightly bound $K$-shell of the material. As a result of this energy transfer it is possible for the electron to be ejected with an energy given by equation 2.1 :

$$
E_{e-}=h \nu-E_{B . E}
$$

where $h$ is Planks constant, $\nu$ is the frequency of the incident radiation and $E_{B . E}$ is the binding energy of the atomic electron. The ejected electron then propagates through the material with a typical range of tens of microns for electron energies up to $100 \mathrm{keV}$ [20]. Furthermore the ejection of the electron results in a vacancy in the electron shell and leaves the atom in an excited state. The vacancy is quickly filled by electrons from the less bound outer shells de-exciting to the vacancy. The de-excitation will result in emission of characteristic fluorescence X ray or Auger 
electrons.

There is no single analytical expression for the probability for photoelectric absorption $\left(\tau_{P E}\right)$ to occur. However a rough approximation is shown in equation 2.2 where $Z$ is the atomic number of the material, the exponent $n$ varies between 3 and 4, $C$ is a constant dependent on detector material and $E_{\gamma}$ is energy of the interacting photon. Photoelectric absorption is a favoured interaction for X-ray detectors, therefore the large dependence of equation 2.2 on the $Z$ value of the material shows that materials with a high $Z$ number such as $\mathrm{CdZnTe}$ are desirable as X-ray and $\gamma$-ray detectors.

$$
\tau_{P E} \sim C \frac{Z^{n}}{E_{\gamma}^{3.5}}
$$

\subsubsection{Compton Scattering}

The second interaction involving $\mathrm{X}$ rays and $\gamma$ rays with matter is Compton scattering. In this interaction the incoming electromagnetic radiation scatters off a 'nearly-free' electron resulting in energy being transferred to the 'nearly-free' electron. If the electron is assumed to initially be at rest the photon scatters through an angle $\theta$ and the recoil electron through an angle $\Phi$ as shown in figure 2.2.

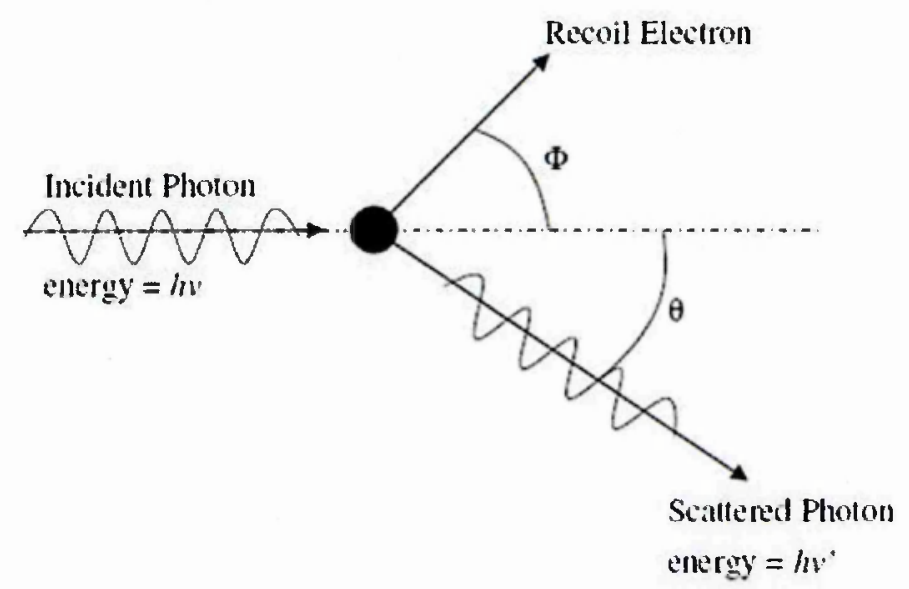

Figure 2.2: Illustration of Compton scattering. A photon of energy $E_{\gamma}$ interacts with a 'nearly-free' electron. The photon scatters through an angle $\theta$ and the electron recoils through an angle $\Phi$. 
Utilising the conservation of total - mass energy and linear momentum, equation 2.3 is formulated to calculate the energy of the scattered photon $E_{\gamma}^{\prime}$. Where $E_{\gamma}$ is the initial photon energy and $\theta$ is the scattering angle of the photon. Energy detected by the detector is the energy of the recoil electron $\left(E_{e}-\right)$ which is the difference between the initial photon energy $\left(E_{\gamma}\right)$ and the scattered photon energy $\left(E_{\gamma}^{\prime}\right)$.

$$
\begin{gathered}
E_{\gamma}^{\prime}=\frac{E_{\gamma}}{1+\frac{E_{\gamma}(1-\cos \theta)}{m_{e} c^{2}}} \\
E_{e}-=E_{\gamma}-E_{\gamma}^{\prime}
\end{gathered}
$$

As this interaction mechanism is dependent on the number of 'nearly-free' electrons the probability for the interaction to occur $\left(\tau_{c s}\right)$ will increase with atomic number of detector material. The variation in the probability for the interaction to occur as a function of energy can be seen in figure 2.3.

\subsubsection{Pair Production}

The third interaction mechanism is relevant only for high energy $\gamma$ rays. In the presence of a Coulomb field from an atom to conserve momentum it is possible for the incident electromagnetic radiation to be absorbed and to be converted into an electron-positron pair. In order for energy to be conserved equation 2.5 must hold, where $E_{\gamma}$ is the initial photon energy, $T_{+}$and $T_{-}$are the kinetic energies of the positron and electron respectively. From equation 2.5 there is an obvious energy threshold below which the interaction cannot occur which is $2 m_{e} c^{2}, 1.22 \mathrm{MeV}$.

$$
E_{\gamma}=T_{+}+m_{e^{+}} c^{2}+T_{-}+m_{e^{-}} c^{2}
$$

There is no simple expression for the probability for pair production to occur $\left(\tau_{\text {pair }}\right)$, however its magnitude is found to vary square of the absorber atomic number $\left(Z^{2}\right)$ $[3,19]$. Furthermore it is the dominant mechanism for high energy gamma rays as shown in figure 2.1 . 


\subsubsection{X-ray and $\gamma$-ray Attenuation Coefficient}

When an $\mathrm{X}$ ray or $\gamma$ ray enters a detector the photons are absorbed or scattered via the interaction process previously explained in sections 2.1.1-2.1.3. It is possible to define the probability per unit length that a specific interaction process will occur, furthermore the sum of the probabilities is known as the linear attenuation coefficient $(\mu)$ and has the following equation:

$$
\mu=\tau_{\text {photoclectric }}+\tau_{\text {compton }}+\tau_{\text {pair }}
$$

where $\tau_{\text {photoelcctric }}, \sigma_{\text {compton }}$ and $\kappa_{\text {pair }}$ are respectively the probability per unit length for photoelectric absorption, Compton scattering and pair production to occur.

At a given X-ray energy it is possible to calculate the number of photons which will be attenuated by a detector by using equation 2.7. Where $I_{0}, I$ are initial and resulting intensity of the radiation respectively and $t$ is the detector thickness.

$$
I=I_{0} e^{-\mu t}
$$

One limitation of the linear attenuation coefficient is that the value varies with the density $(\rho)$ of the material. Therefore the value of the coefficient varies with the physical state of an absorber so for example it will be different for water in its liquid and vapour form. To account for this the mass attenuation coefficient $(\mu / \rho)$ is defined as the linear attenuation coefficient divided by the density of the material. Figure 2.3 shows the mass attenuation coefficient of $\mathrm{Cd}_{0.9} \mathrm{Zn}_{0.1} \mathrm{Te}$ and the probability for an interaction mechanism to occur as a function of energy. As shown the dominant interaction mechanism is highly dependent on the incident radiation energy.

There are discontinuities at energies of $26.7 \mathrm{keV}$ and $31.8 \mathrm{keV}$ in figure 2.3. These energies correspond to the $K$-shell binding energies of $\mathrm{Cd}$ and Te respectively. At photon energies greater than these binding energies it is possible for fluorescence $\mathrm{X}$ ray to be emitted from $\mathrm{Cd}$ and $\mathrm{Te}$. The process resulting in the emission fluorescence $\mathrm{X}$-rays and the effects these X-rays have on the properties of a pixelated X-ray detector will be explained in the next section 


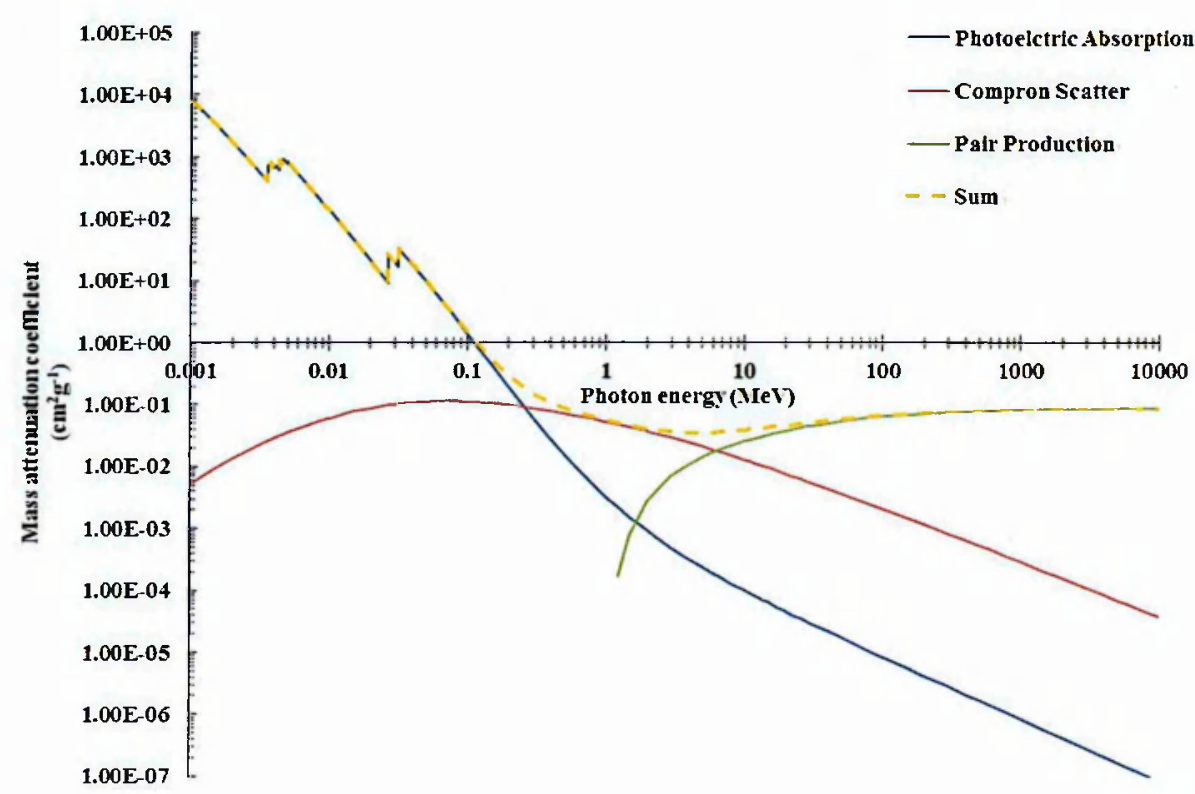

Figure 2.3: Mass attenuation coefficients for the three major electromagnetic radiation interactions with $\mathrm{Cd}_{0.9} \mathrm{Zn}_{0.1} \mathrm{Te}$, calculated using XCOM [4].

\section{$2.2 \quad$ X-ray Fluorescence}

As explained in section 2.1.1 it is possible via photoelectric absorption for an electron to be ejected from its atomic orbit shell leaving the atom in an excited state. The subsequent de-excitation can result in characteristic fluorescence X-rays or auger electrons being emitted. The percentage of the events resulting in fluorcscence Xrays is defined by the fluorescence yield which is $84 \%, 47 \%$ and $88 \%$ for $\mathrm{Cd}, \mathrm{Zn}$ and Te respectively [21].

The process for the emission of fluorescence X-rays is shown in figure 2.4. Typically an electron is emitted from the $K$-shell of an atom leaving the atom in an excited state. Electrons from higher electron orbits subsequently de-excite into the $K$-shell and a characteristic $\mathrm{X}$ ray is emitted with an energy equal to the difference of the binding energies of the electron shells. Figure 2.4 also shows the name for each of the fluorescence X-rays where for example the de-excitation of an electron from the $L$-shell to the $K$-shell is known as a $K_{\alpha} \mathrm{X}$ ray.

Fluorescence X-rays have an effect on the mass attenuation coefficient of a material. As shown in figure 2.5 there are a series of discontinuities in the graph which are due 


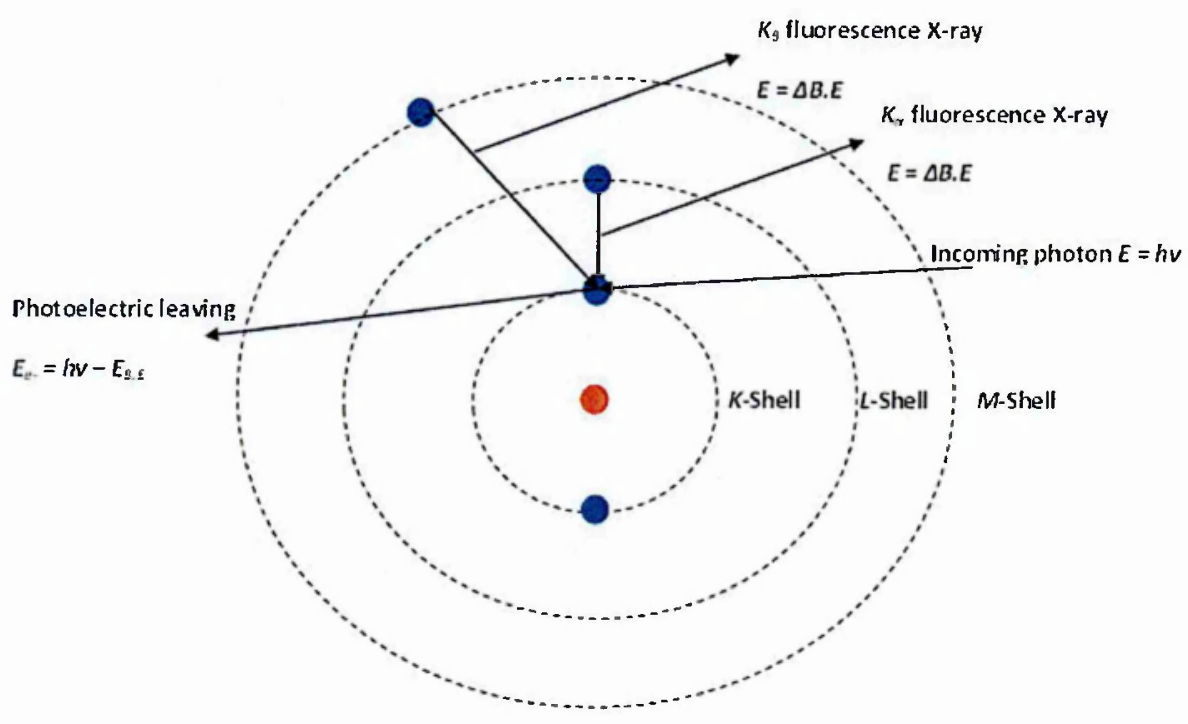

Figure 2.4: Schematic of electron orbitals of an atom and the resulting fluorescence $\mathrm{X}$-rays emitted after the ejection of an electron via photoelectric absorption.

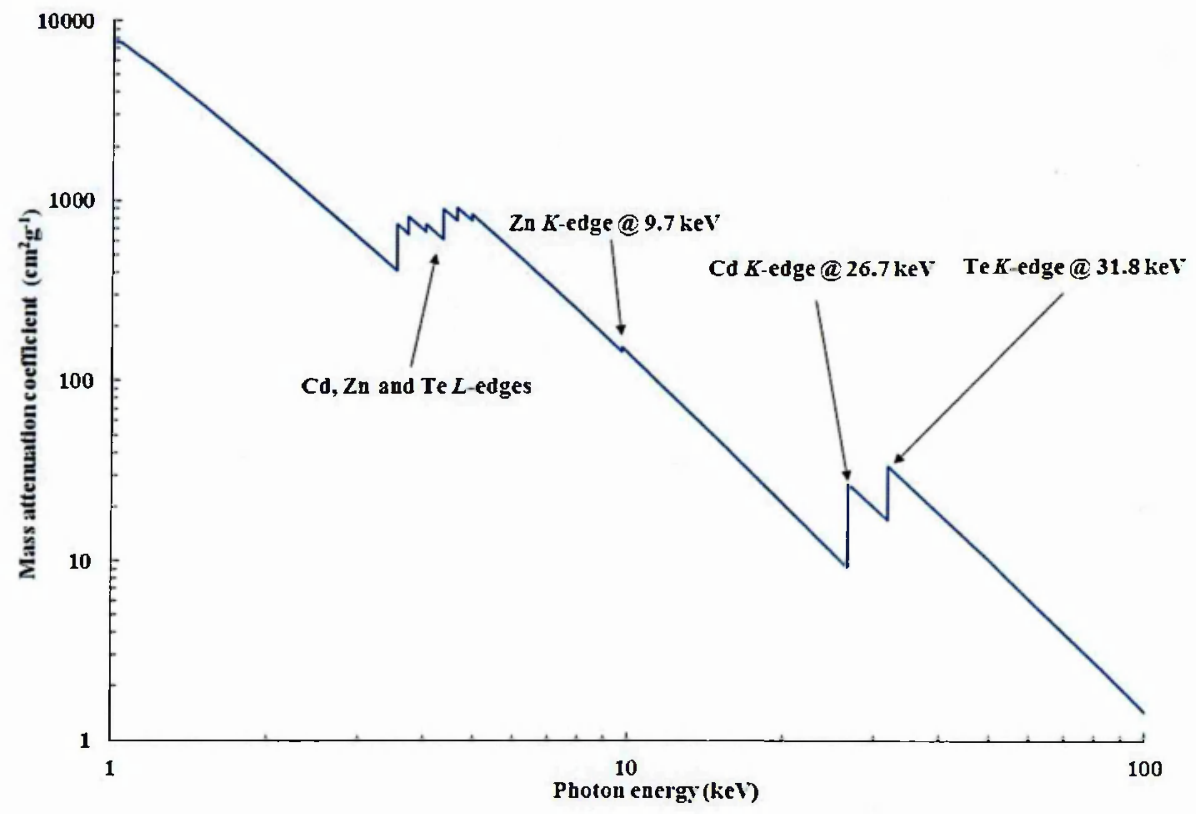

Figure 2.5: Mass attenuation coefficient of $\mathrm{Cd}_{0.9} \mathrm{Zn}_{0.1}$ Te showing the discontinuities due to the binding engines of specific electron orbitals in $\mathrm{Cd}$, $\mathrm{Zn}$ and Te, calculated using XCOM [4]. 
to the binding energies of specific electron orbits in $\mathrm{Cd}, \mathrm{Zn}$ and Te. For example the discontinuities at energies of $26.7 \mathrm{keV}$ and $31.8 \mathrm{keV}$ relate the the binding energies of the $K$-shells of $\mathrm{Cd}$ and Te. At incident photon energies greater than these energies it is possible for electrons to be enitted from these shells resulting in characteristic $K_{\alpha}$ and $K_{\beta}$ fluorescence X-rays being emitted in the subsequent de-excitation process.

\begin{tabular}{|c|c|c|c|c|}
\hline Element & X ray & Energy $(\mathrm{keV})$ & $\lambda_{\mathrm{Si}}(\mu \mathrm{m})$ & $\lambda_{\mathrm{CdZnTe}}(\mu \mathrm{m})$ \\
\hline \multirow{2}{*}{$\mathrm{Si}$} & $K_{\alpha}$ & 1.74 & 1.2 & N/A \\
\cline { 2 - 5 } & $K_{\beta}$ & 1.87 & 0.1 & N/A \\
\hline \multirow{2}{*}{$\mathrm{Cd}$} & $K_{\alpha}$ & 23.18 & N/A & 116.7 \\
\cline { 2 - 5 } & $K_{\beta}$ & 26.10 & N/A & 160.6 \\
\hline \multirow{2}{*}{$\mathrm{Zn}$} & $K_{\alpha}$ & 8.63 & N/A & 8.7 \\
\cline { 2 - 5 } & $K_{\beta}$ & 9.57 & N/A & 11.4 \\
\hline \multirow{2}{*}{$\mathrm{Te}$} & $K_{\alpha}$ & 27.47 & N/A & 67.0 \\
\cline { 2 - 5 } & $K_{\beta}$ & 31.00 & N/A & 91.6 \\
\hline
\end{tabular}

Table 2.1: Attenuation lengths of $K$-shell fluorescence X-rays in $\mathrm{Si}$ and $\mathrm{CdZnTe}$, (values calculated from $[4]$ ).

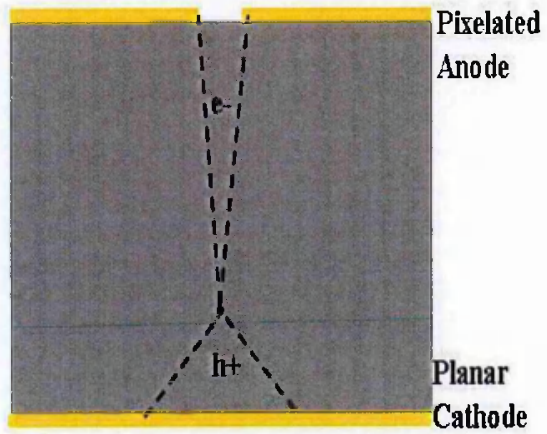

(a) $E<26.7 \mathrm{keV}$

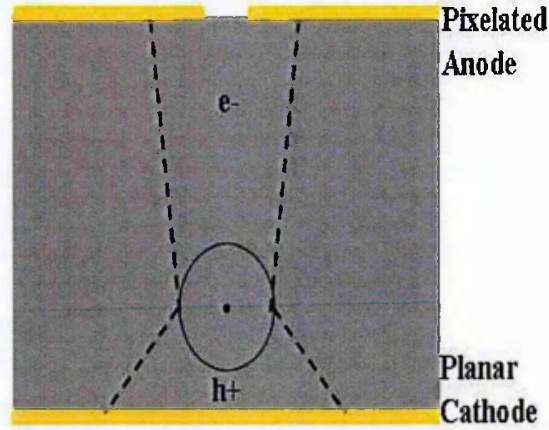

(b) $E \geqslant 26.7 \mathrm{keV}$

Figure 2.6: Schematic representation of the charge cloud evolution in a CdZnTe $\mathrm{X}$-ray detector and the increase in the initial charge cloud size due to the creation of $K$-shell fluorescence X-rays.

Fluorescence X-rays have an effect on the recorded performance and properties of a pixelated radiation detector. When a fluorescence X-ray is emitted it propagates an average distance cqual to the attenuation length $(\lambda)$ of the X-ray in the detector material. As shown in table 2.1 the attenuation length of $\mathrm{Cd}$ and $\mathrm{Te} K_{\alpha}$ and $K_{\beta} \mathrm{X}$ rays is large in CdZnTe compared to the attenuation length of $\mathrm{Si} K_{\alpha}$ and $K_{\beta}$ X-rays in silicon. This larger attenuation length will increase the initial charge cloud size 
and result in a larger charge cloud size (see figure 2.6 for schematic of this process). This increased charge cloud size will result in more charge sharing between adjacent pixels which has a detrimental effect on the detector performance.

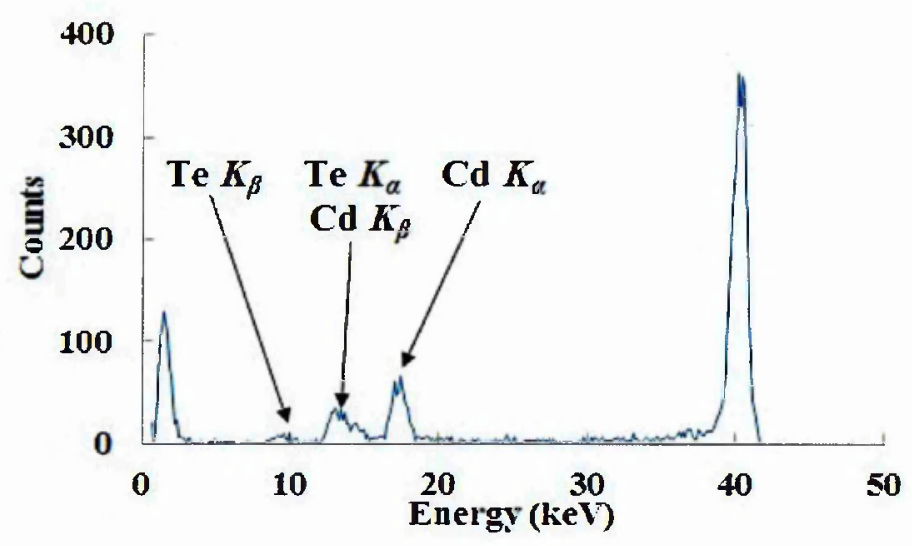

Figure 2.7: Spectrum collected by a CdZnTe detector show the escape peaks at energies less than the incident photon energy of $40 \mathrm{keV}$.

The attenuation length of the fluorescence X-rays can also result in additional peaks being produced by a radiation detector. If the incident X-ray interacts at a position near the edge of the detector it is possible for the emitted fluorescence X-ray to escape the detector medium and subsequently not be collected. The escape of the photon results in peaks at an energy equal to the difference in the incident photon energy and the fluorescence X-ray energy. For example figure 2.7 shows a spectrum collected by a Redlen CdZnTe detector when a $40 \mathrm{keV}$ X-ray beam was indecent on the detector. The photopeak is clearly seen at $40 \mathrm{keV}$, however there are peaks in the region of $9-18 \mathrm{keV}$ which are due to the creation and subsequent escape of $\mathrm{Cd}$ and $\mathrm{Te} K_{\alpha}$ and $K_{\beta}$ X-rays.

\subsection{Semiconductor Band Structure}

The crystalline structure of a material has a large effect on the properties of the material. At a temperature of $0 K$ all the electrons inside a material will be bonded to the atoms on a crystal lattice site. In this situation the electrons are defined to be in the valence band of the material. If energy is transferred to the electrons it 
is possible for them to break frce from the lattice site and thus create an electronhole pair which subsequently propagate through the material. The electrons in this situation are in the conduction band of the material and the holes remain in the valence band.

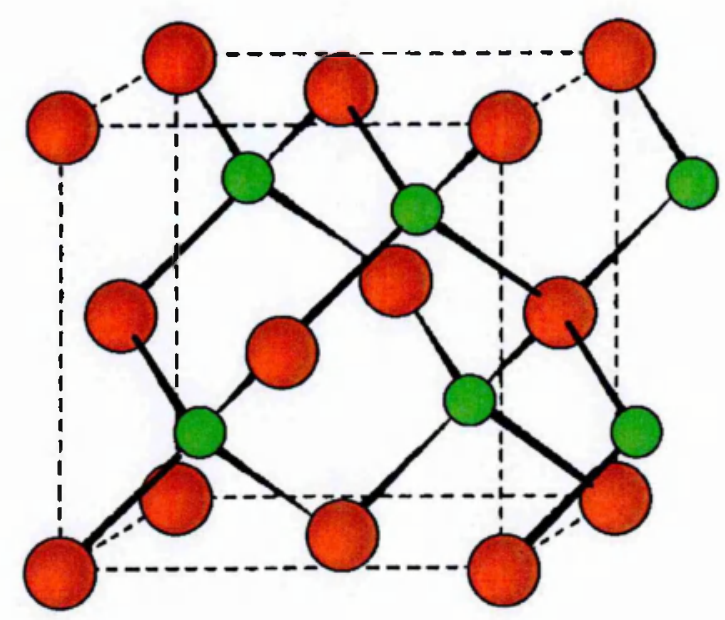

Figure 2.8: The Zinc Blende structure of the compound semiconductor ZnS [22].

The band gap of a material is the energy separation between the top of the valence band and the bottom of the conduction band of a material, which is determined by the crystalline structure of a material. For example CdTe has a crystalline structure of two inter penetrating face centred cubic (fcc) latices one populated with $\mathrm{Cd}$ the other with Te. This crystalline structure is known as a 'Zinc Blende Structure' (see figure 2.8) and results in periodic arrangement of atoms which subsequently creates the band gap of the material.

The band gap of a material can be classified in relation to the band structure of a material. Figure 2.9 shows the band diagram which is a plot of crystal momentum against energy of two different semiconductors. Figure 2.9(a) is a material which is known as a direct band gap material. In this case the top of the valence band and the bottom of the conduction band have the same momentum value. Therefore to create an electron-hole pair an electron in the valence band only needs to be given a very small amount of momentum.

However as shown in figure 2.9(b) for some materials the maximum energy of the 


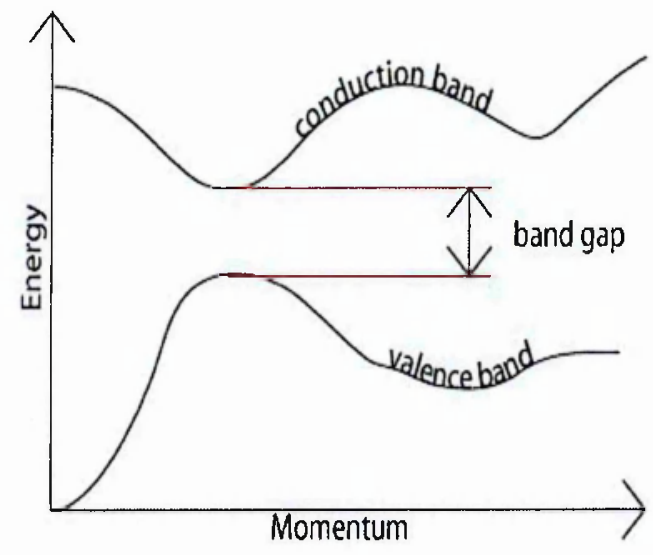

(a) Direct band gap

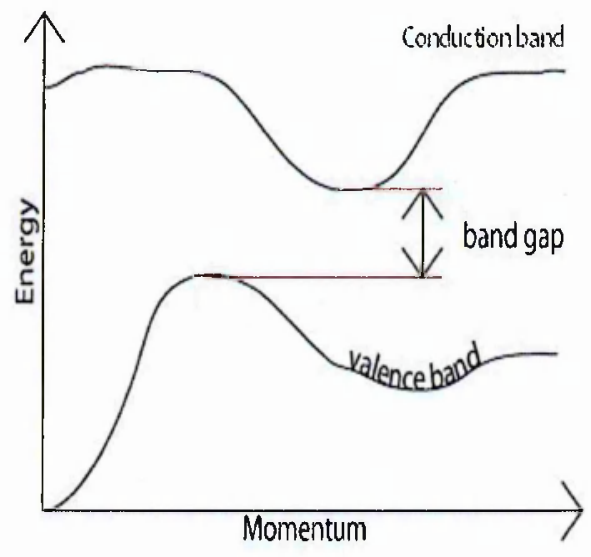

(b) Indirect band gap

Figure 2.9: Band structure of direct and indirect band gap materials [23].

valence band has a different value of momentum compared to the minimum energy of the conduction band. In this case for an electron-hole pair to be created the electron needs to interact with a photon to gain energy and also with a lattice vibration known as a phonon in order to gain or lose momentum. These interaction processes involved with the creation of electron-holes pairs takes longer for an indirect band gap material compared to a direct band gap material. This means direct band gap materials such as CdTe and CdZnTe are preferred as X-ray detector materials.

One way the band gap energy effects the properties of a detector is its effect on the thermal generation of the electron-hole pairs. The probability for an electron-hole pair to be created as a function of temperature $(p(T))$ is given by equation $2.8,[3]$ :

$$
p(T)=C T^{3 / 2} \exp \left(\frac{-E_{g}}{2 k_{B} T}\right)
$$

where $T$ is the absolute temperature, $C$ is a material dependant constant, $k_{B}$ is Boltzmann's constant and $E_{g}$ is the band gap energy.

The number of thermally generated carriers is important for detectors as it has a significant effect on the bulk leakage current. As the band gap decreases the number of thermally generated carriers increases this effect in conjunction with a lower resistivity for smaller band gap materials results in a higher leakage current. This is an undesirable effect of small band gap materials which resulted in more 
research being undertaken on large band gap materials and there use as a radiation detector.

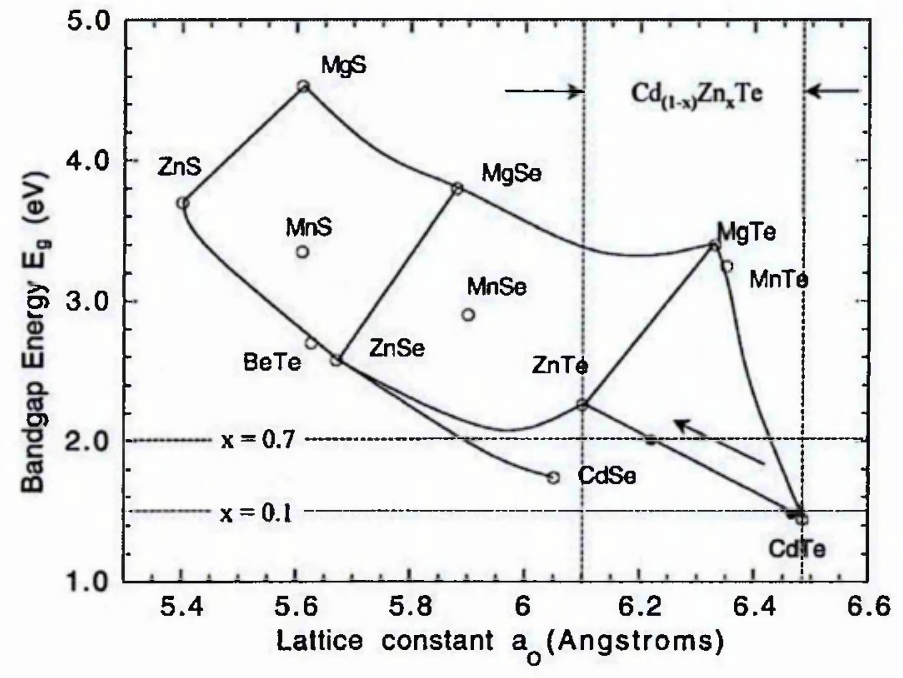

Figure 2.10: The band gap of II/VI semiconductors as function of lattice constant [24].

One major advantage of compound semiconductors like CdZnTe compared to elemental semiconductors like silicon is that the material properties such as the band gap can be changed to fit industrial needs. At room temperature CdTe has a band gap of $1.51 \mathrm{eV}$ which is determined by average inter-atomic spacing between the $\mathrm{Cd}$ and $\mathrm{Te}$ atoms. If a fraction $(x)$ of the $\mathrm{Cd}$ atoms are replaced with $\mathrm{Zn}$ atoms the inter-atomic distance will dccrease and therefore the band gap will increase. Figure 2.10 shows a variety of different II/VI semiconductors and the effect the lattice constant has on the room temperature band gap. For the specific case of $\mathrm{Cd}_{1-x} \mathrm{Zn}_{x} \mathrm{Te}$ equation 2.9 can be used to calculate the band gap as a function of the blending fraction $(x)$ of $\mathrm{Zn}[25]$.

$$
E_{g}(x)=(1.510 \pm 0.005)+(0.606 \pm 0.010) x+(0.139 \pm 0.010) x^{2}
$$




\subsection{Generation of Charge Carriers}

When radiation enters a detector it results in the detector material becoming ionized. In the case of a direct band gap semiconductor material electrons are raised to the conduction band of a material and holes are left in the valence band, in the case of indirect semiconductors phonons are needed to change the momentum of the electrons making it possible for the electrons to be excited to the conduction band. The number of electron-hole pairs produced by the incoming radiation is dependent on a specific property of the material, the electron-hole pair creation energy otherwise known as the $W$-value. The $W$-value is the energy in $e V$ required to create one electron-hole pair which is largely independent of the energy of the ionising radiation. Figure 2.11 shows a variety of materials and the relationship between the $W$-value and the band gap of the material. It is shown that a large number of the materials lie on a line with the equation shown in the equation 2.10 where the offset of the linear fit is due to the role of phonon's in the creation of charge carriers. This means it is possible to calculate the $W$-value of a material if its band gap is known.

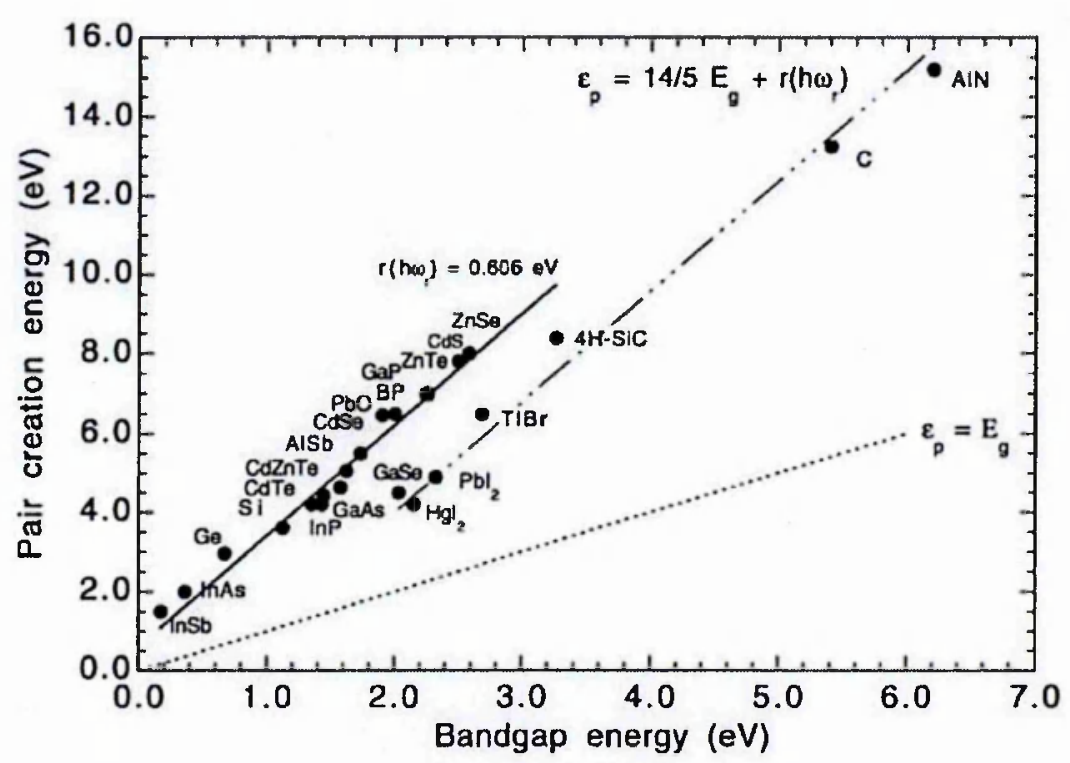

Figure 2.11: Electron-hole pair creation energy ( $W$-value) as a function of band gap for variety of different materials [24]. 


$$
W(e V)=\frac{14}{5} E_{g}(e V)+0.606(e V)
$$

If the electron-hole pairs are created in the presence of an electric field they will drift towards their respective attracting electrodes. The drifting of the charge results in a current being induced on the electrode which can be integrated into a charge pulse. The resulting amplitude of the integrated charge will be therefore directly dependent on the $W$-value of a material.

The number of electron-hole pairs $N$ created can be simply calculated using equation 2.11. If it is assumed that the creation of each electron-hole pair is an independent event the process can be modelled with a Poisson distribution. This means the assumed variance $\left(\sigma^{2}\right)$ of the number of electron-hole pairs created is defined in equation 2.12 .

$$
\begin{aligned}
N & =\frac{E}{W} \\
\sigma^{2} & =\frac{E}{W}
\end{aligned}
$$

However it is found that the creation of an electron-hole pair is not an independent event which results in a smaller variance [3]. Therefore a factor called the Fano factor $(F)$ is introduced to relate the observed variance when all other sources of noise are removed and the assumed Poisson variance as shown in equation 2.13.

$$
\sigma^{2}=\frac{F E}{W}
$$

\subsection{The Shockley-Ramo Theorem}

The common principle of any semiconductor radiation detector is that when ionizing radiation interacts with the detector material a number of electron-hole pairs are created. In the presence of an electric field these charge carriers drift towards their respective attracting electrodes. This drifting induces a current pulse which can be 
integrated in an external circuit to form a charge pulse which is proportional to the energy of the incident radiation.

In the 1930's a theorem was developed independently by W.Shockley [26] and S.Ramo [27] to model the induction of charge in detectors with complex electrode geometries. The theory was initially formulated for vacuum tube geometries which was subsequently extended to semiconductor radiation detectors [28-30]. (A comprehensive summary of Shockley-Ramo theory is by Z.He [12]).

The Shockley-Ramo theorem states that the current $(i)$ and the subsequent integrated charge $(Q)$ induced on an electrode by moving charge is given by:

$$
\begin{gathered}
i=q \vec{v} \cdot \vec{E}_{0}(x) \\
Q=-q \Delta \psi_{0}(x)
\end{gathered}
$$

where $\vec{v}$ is the instantaneous velocity of charge $q . \psi_{0}(x)$ and $\vec{E}_{0}(x)$ are the so called 'weighting potential' and 'weighting field' at the position $x$ of the moving charge where the weighting field is defined as:

$$
\vec{E}_{0}(x)=\frac{\partial \psi_{0}(x)}{\partial x}
$$

The weighting potential should not be confused with the electronic potential which describes the velocity and trajectory of the moving charge. The weighting potential represents the electrostatic coupling between moving charges and the induced charge on an electrode and is defined as "the potential at a position $x$ when the selected electrode is at unit bias, $1 \mathrm{~V}$, and all others are at $0 \mathrm{~V}$ " [12] and is dependent on the carrier motion and the electrode design.

The simplest example of a detector is to consider an intrinsic material (i.e. contains no space charge) between two infinitely large planar electrodes. As shown in figure 2.12(b) the weighting potential is linear in nature from 0 at the cathode to 1 at the anode. As shown in equation 2.17 it is possible to calculate the charge induced on the anode when $N$ charge carriers have been created from the interaction of ionizing 


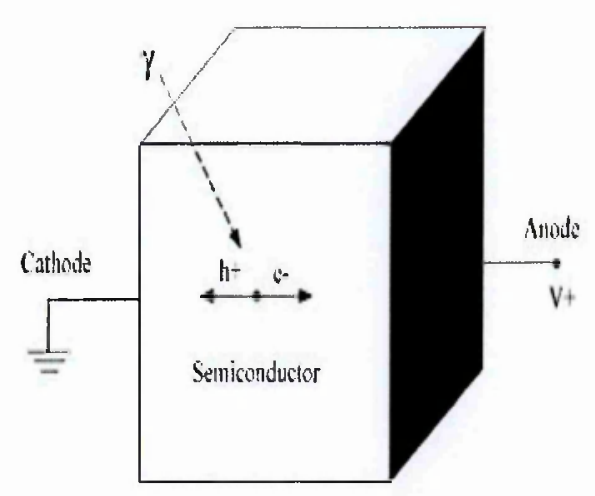

(a) Direct band gap

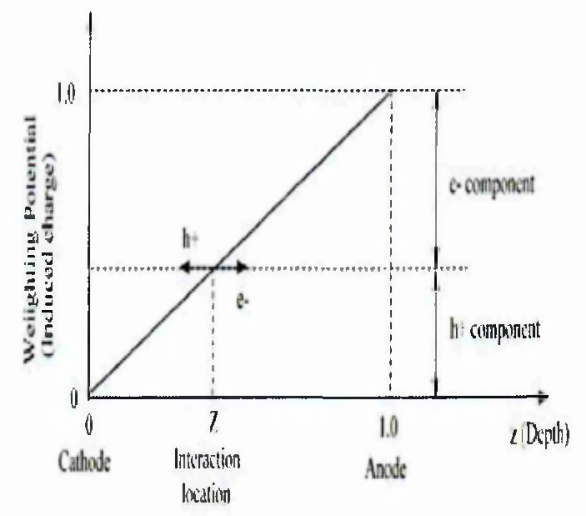

(b) Indirect band gap

Figure 2.12: (a) Schematic of a simple radiation detector with infinite planar cathodes and (b) its resulting weighting potential for the anode [12].

radiation at a position $Z$ in figure $2.12(\mathrm{~b})$.

$$
\begin{aligned}
Q & =-q \Delta \psi_{0}(z) \\
& =-N e\left[\psi_{0}(0)-\psi_{0}(Z)\right]_{\text {hole }}-N(-e)\left[\psi_{0}(1)-\psi_{0}(Z)\right]_{\text {electron }} \\
& =N e
\end{aligned}
$$

Equation 2.17 shows that if the mean free path $(\lambda)$ of the charge carriers are long compared to the detector thickness the charge induced on the anode will be proportional to the energy of the incident radiation. As shown in equation 2.11 this is due to the number of electron hole pairs created $N$ is proportional to the energy $E$.

For CdZnTe the mean free path of holes is much smaller compared to electrons which can result in the incomplete collection of the holcs. As a result of this the charge on the planar anode will have a range of values as shown in equation 2.18. This will result in the induced charge not being proportional to the energy of the incident radiation and this will degrade the recorded detector performance.

$$
Q=N e\left[1-\psi_{0}(x)\right]
$$




\subsection{Single Charge Carrier Sensitive Detectors}

As shown in figure 2.13(a) [31] the poor hole transport properties of CdZnTe and the resulting degradation in detector performance by "hole-tailing" is a low energy broadening of the photopeak. Many techniques have been utilized to account for the poor hole transport properties of CdZnTe. Pulse discrimination has been used to remove the slow pulses (trapped holes) from the resulting spectrum and therefore only the fast pulses (electron movement) are used [31-34]. As shown in figure 2.13(b) the recorded spectroscopic performance the detector improves compared to the spectrum without the rejection of slow pulses [31]. However the major disadvantage of this technique is that it is not fundamentally solving the issue of poor hole transport and results in a drop in efficiency which is undesirable for many applications.

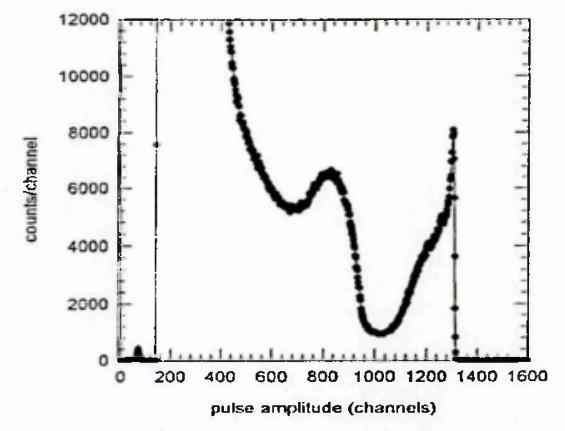

(a) Without pulse rejection

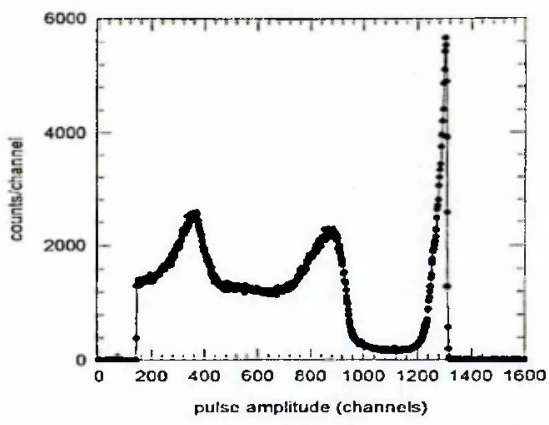

(b) With pulse rejection

Figure 2.13: The effect the rejection of slow rise time pulses has on the recorded spectroscopic performance of a ${ }^{137} \mathrm{Cs}$ spectrum taken by a $1.8 \times 1.8 \times 0.5 \mathrm{~cm} \mathrm{Cd}_{0.9} \mathrm{Zn}_{0.1} \mathrm{Te}$ detector [31].

As a result of the poor hole transport much research has been undertaken on single charge carrier sensitive detectors. Through complex electrode designs the weighting potential can be modified so the detector is only sensitive to one charge carrier. One such example is a detector with a planar cathode and anode consisting of two co-planar grids $[35,36]$.

Figure 2.14(a) shows a schematic of a co-planar detector, if a small bias is applied across the two electrodes when charge is created the electrons will drift towards the grids and be collected by electrode 2 . As the electron charge cloud drifts it will 


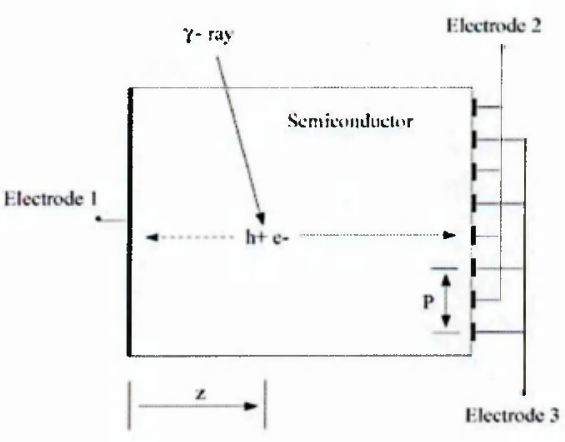

(a)

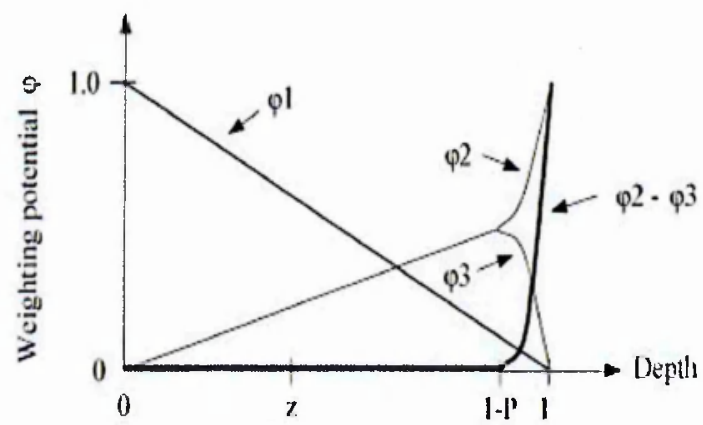

(b)

Figure 2.14: (a) A schematic of a co-planar grid single charge carrier sensitive detector, (b) the weighting field for the three electrodes. The subtracted signal from electrodes 2 and 3 is predominantly due to electron drift close to the coplanar anodes [37].

experience the weighting potentials of $\varphi_{2}$ and $\varphi_{3}$. However if the difference in output of charge sensitive preamplifiers is calculated the resulting weighting potential will be $\varphi_{2}-\varphi_{3}$ as shown in figure 2.14(b). In an ideal case where no electron trapping is present the resulting weighting potential will be zero until a region where the depth $(z)$ is equal 1-P where $P$ is the period of the grids. As a result the final signal will be dominated by movement of charge in a region close to the anode i.e. the electron component.

However there are certain limitations of co-planar grid detectors. In some cases it is possible to result in a non-zero weighting potential across the device thus introducing the hole component into the final output. If the output of the two anode outputs are not accurately gain matched the subtraction of the outputs will result in a non-zero valuc. Furthermore in most cases electron trapping takes place which will also result in a non-zero weighting potential across the device [37]. It can also be challenging to fabricate large scale co-planar grid detectors, finally if the period $(P)$ is small the capacitance and the resulting leakage current between the grids can be large resulting in a decrease in the signal-to-noise ratio [12].

Another example of single charge carrier sensitive detector is a drift strip/ring detector $[38,39]$. Figure 2.15 shows a schematic of a drift strip detector. In between dashed lines $\mathrm{A}$ and $\mathrm{B}$ is a single drift ring cell which consists of 9 separate strips. 


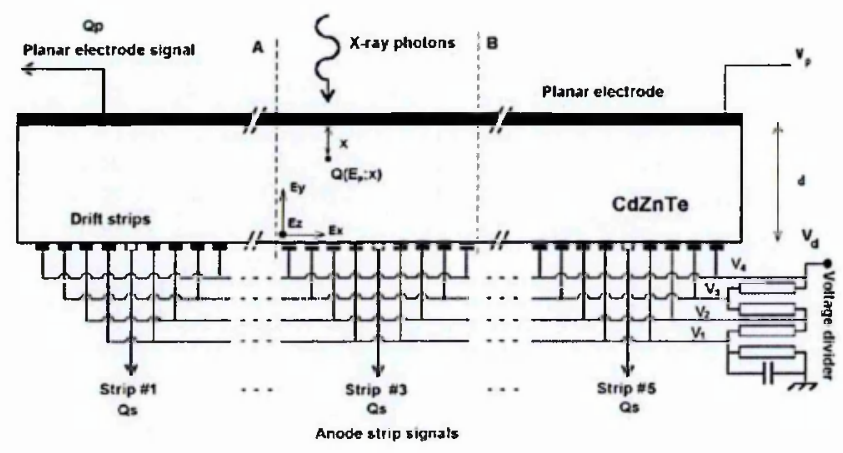

Figure 2.15: Schematic of drift strip detector. One detector cell is inbetween the dashed lines $\mathrm{A}$ and $\mathrm{B}$. The strips are biased so as to focus electrons onto the collecting anode [38].

In the case of the detector used by Kuvvetli et.al. [38] the planar cathode was held at a bias of $V_{P}=-300 \mathrm{~V}$ and the central strip is the anode which is held at a bias of $V_{A}=0 \mathrm{~V}$. The remaining eight strips are the drift strips and are biased by a voltage divider where the bias applied to each strip is equal to $V_{i}=V_{p} \cdot\left({ }^{\imath} / 4\right)$ where $i=1,2,3,4$. The resulting potential focuses the electrons onto the central anode and makes the detector only sensitive to the motion of electrons. A more comprehensive review of co-planar grids, drift strip detectors and other single charge carrier sensitive devices can be found in [12].

Another example of a single carrier sensitive device is one with a planar cathode and an anode with an array of pixels [11]. These small pixel detectors are of interest for this thesis and will be explained in the next section.

\subsubsection{Pixelated CdZnTe Radiation Detectors}

Figure 2.16(a) is a schematic of a detector of a thickness $T$ with a planar cathode electrode and anode of pixels with a width $\varepsilon$. If the movement of generated charge carriers is read out from pixel 1 the weighting potential as seen by the electrons created at point $\mathrm{A}$ is shown in figure 2.16(b).

The weighting potential shown in figure 2.16(b) has the property of being almost 0 until in the vicinity of the pixel where it increases dramatically up to a value of 1 . For example if ionising radiation enters the detector so that charge carriers 


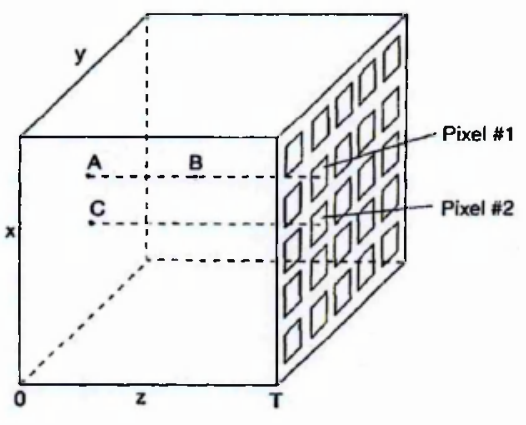

(a)

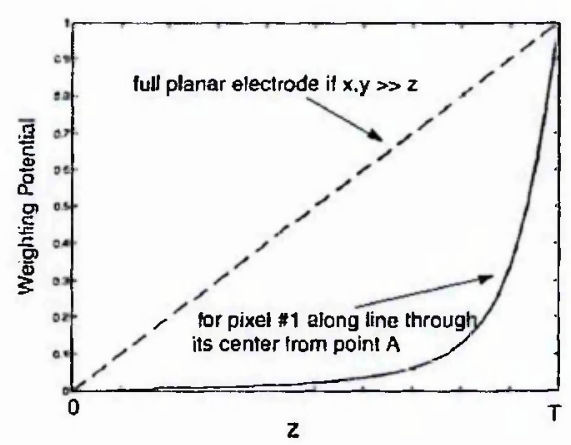

(b)

Figure 2.16: (a) Schematic of a pixelated radiation detector and (b) its resulting weighting potential for signal read from pixel $1[3]$.

are created at point $\mathrm{A}$ in figure 2.16(a) the electrons and holes will drift and be collected by pixel 1 and the planar cathode respectively. As previously explained in section 2.5 the charge induced on an electrode is proportional to the change in the weighting potential. Therefore when the charge is created at point $A$ the change in weighting potential between point $\mathrm{A}$ and the collecting electrode will be negligible for the holes and therefore the subsequent signal generated on pixel 1 is dominated by the motion of the electrons.

The increased gradient in the weighting potential shown in figure 2.16 (b) can be heightened by making the pixel width $\varepsilon$ smaller compared to the detector thickness $T$. As shown in figure 2.17 as the value of the pixel width divided by the detector thickness $(\varepsilon / T)$ decreases the gradient of the weighting potential in the vicinity of the pixel increases. This is known as the 'small pixel effect'.

The detector array used in the project has a pixel size of $200 \mu \mathrm{m}$ and a detector thickness of $2 \mathrm{~mm}$. As a result the ${ }^{\varepsilon} / T$ value of the array is 0.1 . This means the weighting potential of the pixel array will be similar to the one shown in figure 2.17 . This means the 'small pixel effect' will have a significant effect on the performance of the CdZnTe pixelated detector. 


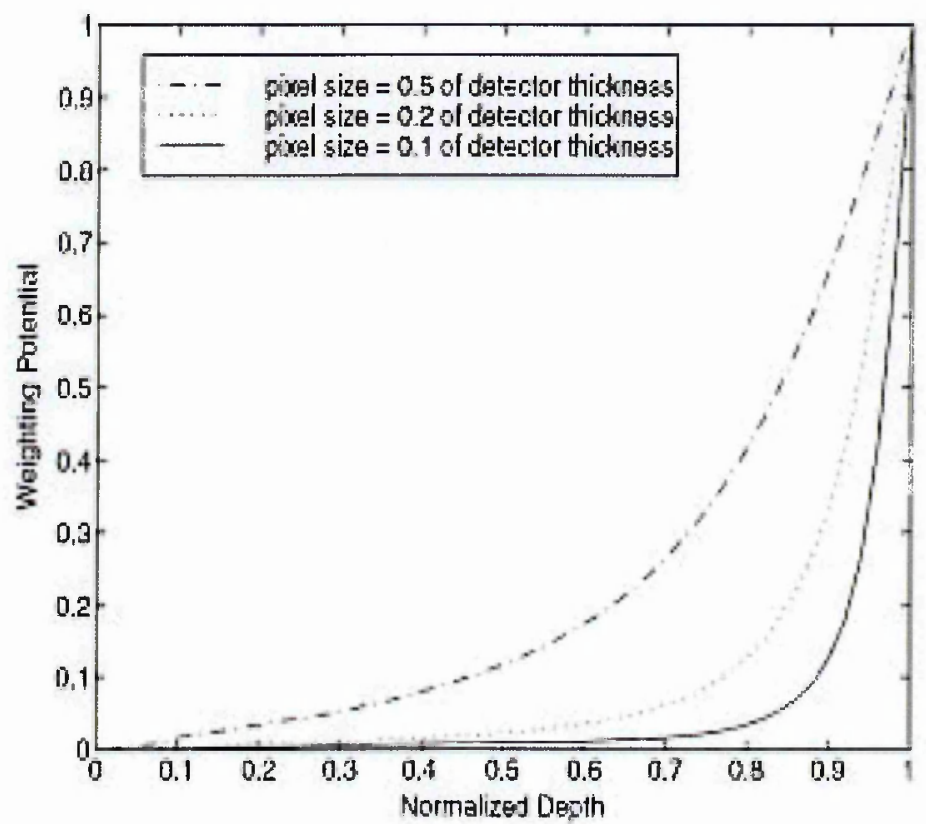

Figure 2.17: Weighting potential as read from a pixel for varying values of $\varepsilon / T$ ' showing the result of the 'small pixel effect' [3].

\subsubsection{Charge Sharing Between Adjacent Pixels}

A major limiting factor of pixelated radiation detectors is that it is possible that charge can be induced on more than one pixel. To understand how this phenomena takes place a more detailed explanation of how charge clouds propagate and evolve as they move across the detector must be undertaken.

As previously explained in section 2.4 when an X-ray interacts with the detector material, electron and hole charge clouds are created with an initial width equal to the propagation length of the electrons and holes, $(10 \mu \mathrm{m}$ at $40 \mathrm{keV}[20,40])$. The initial charge cloud size can also be increased by the creation and propagation of Auger electrons and fluorescence X-rays at incident energies greater than the $K$ shell absorption energy of the detector materials. In the presence of an electric field the charge clouds will propagate to the respective attracting electrodes. During this propagation the charge clouds will diffuse parallel and perpendicular to the propagation direction which have different effects on the resulting signal generated on the collecting electrode. 
The parallel diffusion primarily has an effect on the rise time of the signal generated on the collecting electrode [13]. The perpendicular diffusion has a large effect on the probability that charge will be induced on more than one pixel. It is possible to model the diffusion perpendicular to the propagation direction with a Gaussian distribution. This means the charge cloud radius as a function of detector bias can be calculated as follows:

$$
r=1.15 \sigma=1.15 \sqrt{\frac{2 k_{B} T d D}{e V}}
$$

where $r$ is the charge cloud radius, $d$ is the drift distance of the charge cloud, $D$ is the detector width, $k_{B}$ is Boltzmann's constant, $T$ is the absolute temperature, $e$ is the electronic charge and $V$ is the detector bias [41]. For the detector array used in this project the resulting charge cloud radius is $r=29.5 \mu \mathrm{m}$ at a detector biases of $-300 \mathrm{~V}$ and $r=51 \mu \mathrm{m}$ at a detector bias of $-100 \mathrm{~V}[4]$.

When the charge cloud diameter is comparable to the pixel size the charge cloud can induce a signal on more than one pixel. As a result the signal amplitude corresponding to a full energy event will be shared between adjacent pixels and thus decreasing the resolution of the detector. This can be corrected by summing the resulting signals from adjacent pixels $[15,16]$.
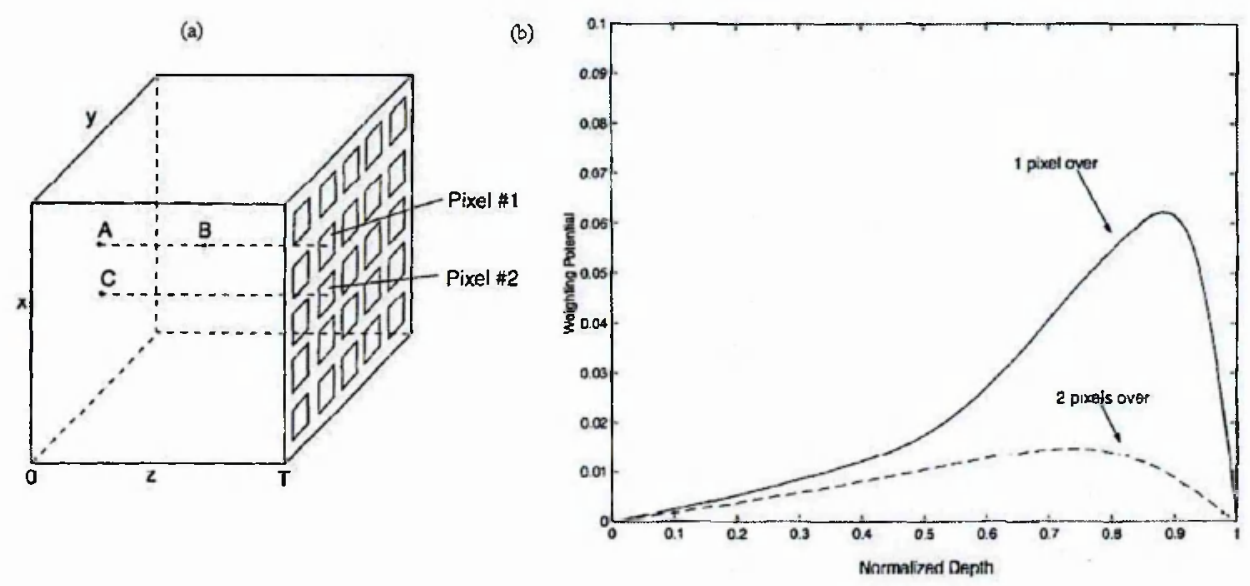

Figure 2.18: (a) Schematic of a pixelated radiation detector, (b) weighting potential of pixels which are adjacent to the main collecting pixel [3].

There is a second and subtlety different way to induce a signal on more than one 
pixel from an interaction of radiation with a detector. Imagine the scenario that $N$ electron-hole pairs are created at point $\mathrm{C}$ which is very near the cathode and in line with pixel 2 on figure 2.18(a). The electrons created by the interaction of the radiation with the detector will follow the actual electric field lines and be collected by pixel 2 .

However as the electron charge cloud propagates across the device it is possible to induce a transient current pulse on an adjacent pixel. Figure 2.18(b) is weighting potential of a pixel which is adjacent to the collecting pixel. As the charge cloud propagates across the device a current pulse is induced on both pixels (see figure 2.19) where the pulse induced on the neighbouring pixel is bipolar in nature. When the current pulses are integrated the induced charge is measured, thus when the current pulse induced on the neighbouring pixel is integrated the total collected charge is zero but a pulse is still induced on the pixel.

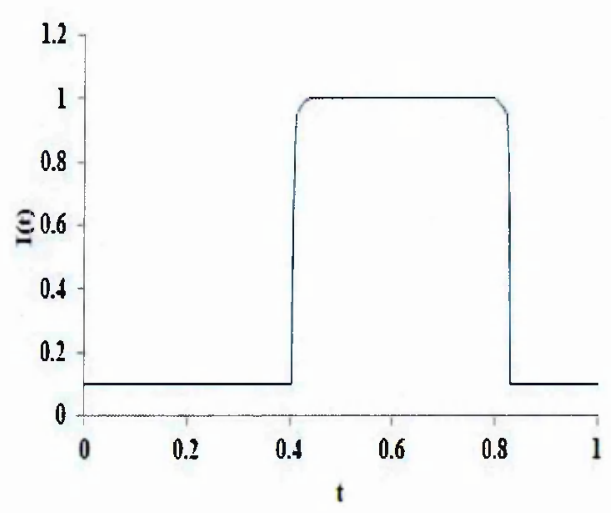

(a) Main Pixel Current Pulse

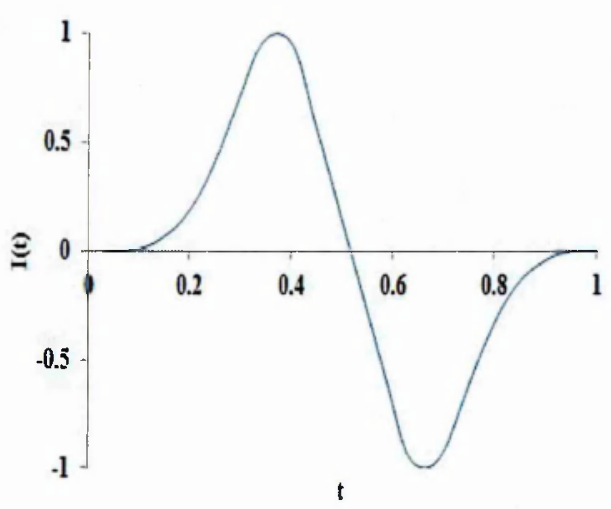

(b) Neighbouring Pixel Current Pulse

Figure 2.19: Schematics of the current pulses induced on the main collecting pixel and an adjacent pixel which results in zero net charge and a transient current pulse is induced on the collecting pixel.

An example of a transient pulse can be seen in figure 2.20 which shows the interaction of a $78 \mathrm{keV}$ X-ray (generated by the I15 beam line at the diamond light source) with one pixel and the subsequent transient pulse induced on a neighbouring pixel. The peaking time when the maximum charge is measured for each pulse is shown in table 2.2. It is found that the transient charge pulse reduces to zero over the time scale 
when the main pixel increases. Thus the measured total collected charge will not be effected by the creation of the transient signal on the adjacent pixel.

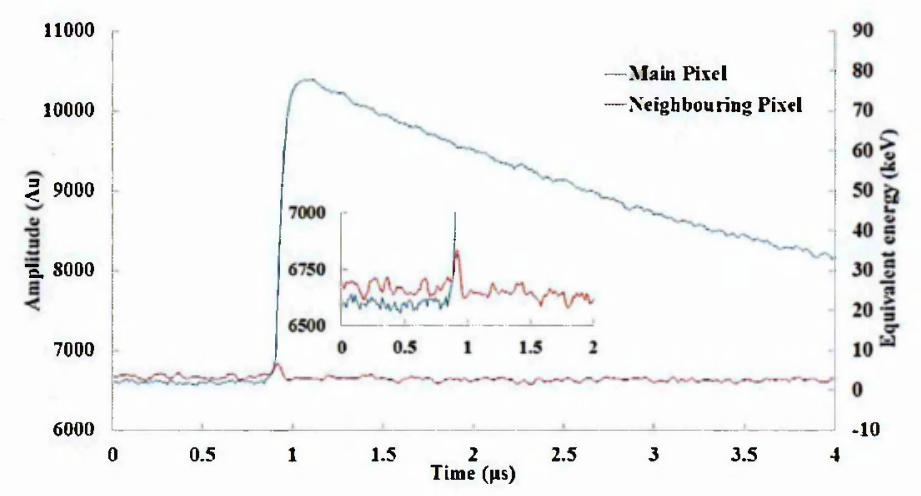

Figure 2.20: Example of transient pulse being generated on a neighbouring pixel. A $78 \mathrm{keV} \mathrm{X}$-ray is collected by a pixel (blue pulse) and a transient pulse is subsequently induced on a neighbouring pixel (red pulse).

\begin{tabular}{|c|c|}
\hline Pixel & Peak Time $(\mu \mathrm{s})$ \\
\hline Main Pixel & 1.07 \\
\hline Neighbouring Pixel & 0.92 \\
\hline
\end{tabular}

Table 2.2: Peaking time of the pulses shown in figure 2.20 showing that the transient pulse evolves and drops to zero over the time when the main pixel pulse is generated. 


\section{Chapter 3}

\section{Experimental Setup and}

\section{Apparatus}

This chapter will discuss the experimental apparatus and procedures used in this project. Initially a brief overview of how gold contacts are fabricated on the CdZnTe material will be given. Furthermore the equipment used to characterise the CdZnTe material before and after the fabrication of the gold pixels will be described. Discussions regarding the PIXIE ASIC and digital pulse processing procedure used in this project will be undertaken. Finally the experimental setup used on the I15 beamline at the Diamond Light Source to investigate charge sharing in pixelated detectors will be explained.

\subsection{Fabrication of CdZnTe Detectors}

A photolithography process is used to fabricate gold pixel contacts onto the $\mathrm{CdZnTe}$ sample [42]. Initially the sample has undergone a mechanical polishing and chemical etching procedure. The sample is mechanically polished with a $3 \mu \mathrm{m}$ Silicon-Carbide pre-prepared slurry then $0.3 \mu \mathrm{m}$ and $0.05 \mu \mathrm{m}$ Aluminium Oxide powders are mixed separately with de-ionized water to create a slurry. This results in the sample having a near mirror finish. Further chemical etching is undertaken in a Bromine $(0.05 \%)$ Methanol $(99.95 \%)$ solution for $60 \mathrm{~s}$ and is then finally placed in Isopropranol and 
Methanol washes [43]. Once this procedure has been completed the gold contacts can be fabricated onto the prepared CdZnTe sample.

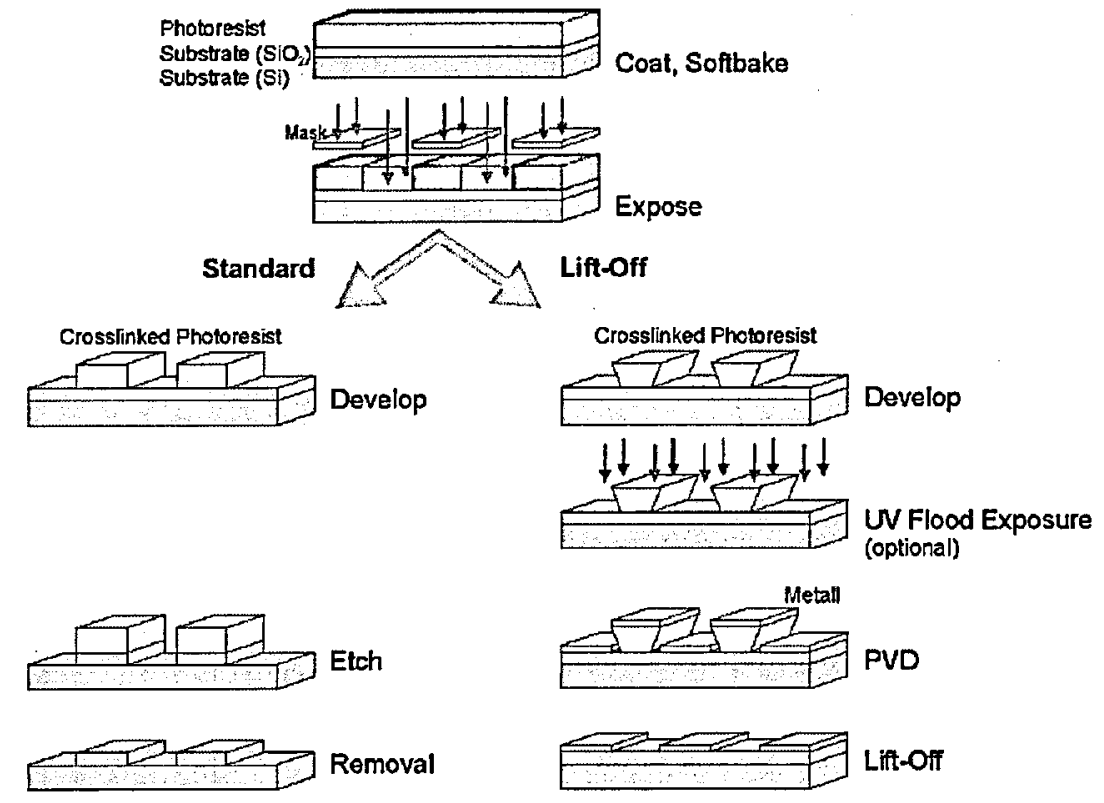

Figure 3.1: Process flow for the creation of gold contact on a CdZnTe sample via the use of ma-N-410 negative photoresist [44].

The photolithography process is shown in figure 3.1. Initially ma-N-410 negative Photoresist [44] is pipetted on top of the sample. The sample is then placed in the centre of a spinner and is attached firmly to the spindle by the use of an external vacuum pump. The sample is then spun for $30 \mathrm{~s}$ at $500 \mathrm{rpm}$ and $30 \mathrm{~s}$ at $3500 \mathrm{rpm}$ resulting in $a \sim 1 \mu \mathrm{m}$ film on top of the sample. The sample is then prebaked for $120 \mathrm{~s}$ on a hotplate set at temperature of $95^{\circ} \mathrm{C}$.

To create the desired pattern the sample is placed in a mask aligner. The sample is placed accurately under the desired pattern by the use of a microscope and moving sample in the all directions via use of stage that can be moved in distances on a micron scale. Once the sample is accurately under the pattern the sample is exposed to UV light for $120 \mathrm{~s}$. The sample is then placed in developer for $60 \mathrm{~s}$ and de-ionized water for $120 \mathrm{~s}$ to stop the reaction.

As shown in figure 3.1 the areas which are not covered by the mask the photore- 
sist reacts with UV light and become insoluble to the developcr. This results in photoresist only being present on the sample where the contacts are not to be fabricated. Figure 3.2 shows an example of a daisy chain test structure after developing. As shown in the figure the photolithography process makes it possible to fabricate structures on a micron scale.

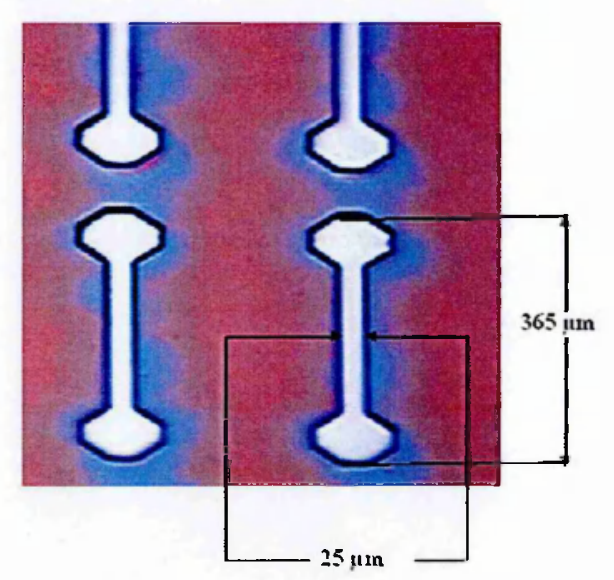

Figure 3.2: High resolution picture of Si sample with daisy chain test structure developed onto the sample before sputtering of gold contacts.

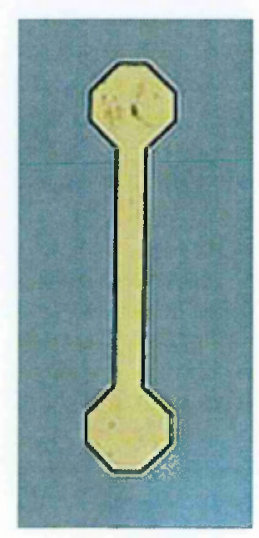

Figure 3.3: Gold test structure after sputtering of gold contacts on Si sample.

To create the gold contacts a physical vapour deposition process is undertaken via the use of a sputterer. A gold target has a current of $65 \mathrm{~mA}$ passed through it resulting in the target becoming a plasma which then makes it possible for gold ions to be ejected from the target onto the sample. If the $\mathrm{CdZnTc}$ is placed directly 
under the target under vacuum the gold ions can be deposited on top of the sample. An onboard Film Thickness Monitor (FTM) is then used to measure the thickness of gold deposited on the sample. The sample is then placed in photoresist remover to carry out a lift-off process of the remaining photoresist. This results in gold only being present where the gold pixels should be as shown in figure 3.3.

\subsection{Surface Characterisation Methods}

Before the fabrication of gold contacts it is desirable to perform metrology studies on the CdZnTe sample. To carry these investigations out a Zygo Newview 200 noncontact optical profiler was used. Furthermore the Zygo and a scanning electron microscope was used to characterize the gold pixels after fabrication.

\subsubsection{Zygo Newview 200}

The Zygo Newview 200 is a non-contact optical profiler based at the Innovations Technology Access Centre (ITAC) at the Rutherford Appleton Laboratory. Figure 3.4(a) is a photo of the Zygo system, it consists of the microscope and external PC installed with the controlling software. The Zygo has a motorized stage where the sample is placed which can be moved in the $X, Y, Z$ and $\theta$ directions.

Figure 3.4(b) is a schematic of how the Zygo works. A white light source is divided into two, directing one onto the sample and one onto an internal reference. Once the light reflects off the sample surface the light will be out of phase with the light collected at the internal reference due to surface irregularities of the sample. This means the subsequent interference fringes can be used to characterize the detector sample.

The Zygo was operated in a stitching mode and the macroscopic curvature of the device was measured. This curvature is important when mounting the final fabricated detector to an ASIC. The Zygo also has the ability to work in higher magnification mode where the surface roughness and the characterization of the fabricated gold pixels can be carried out. 


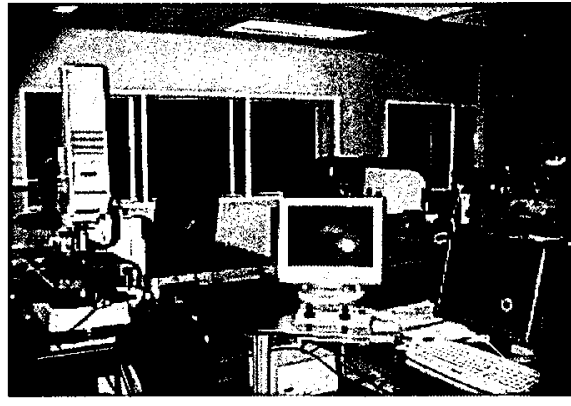

(a)

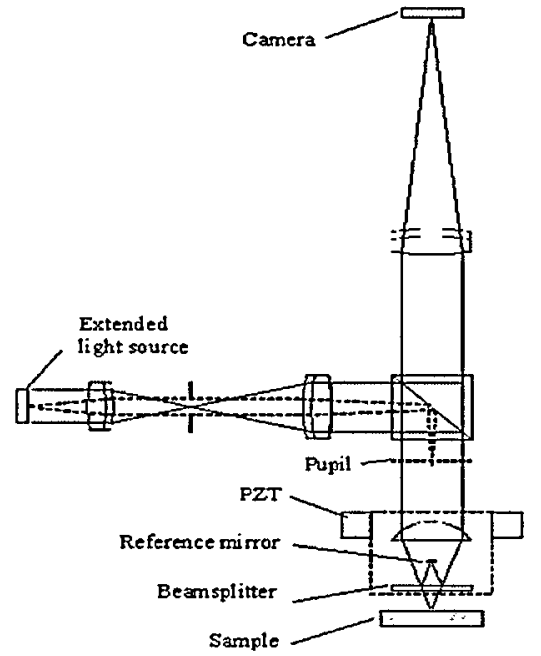

(b)

Figure 3.4: Photo and schematic of the non-contact Zygo Newview 200 optical profiler.

\subsubsection{Scanning Electron Microscope (SEM)}

A Scanning Electron Microscope (SEM) was used to characterize the gold pixels fabricated onto the CdZnTe sample. An SEM is a type of electron microscope that produces images of a sample by scanning it with a focused beam of electrons. When the focused electron beam interacts with electrons inside the sample the energy transfer can result in the emission of electrons via elastic and inelastic scattering and the emission of X-rays. The detection of these electrons and X-rays can then be used to create a highly magnified image of the sample. The SEM in this study was operated at a magnification of 1000 and 6000 times. This made it possible to investigate a whole gold pixel and the subsequent edge of that pixel. This was then used to support the data recorded and analysed on the Zygo Newiview 200 optical profiler.

\subsection{PIXIE ASIC and Readout System}

An Application Specific Integrated Circuit (ASIC) has been developed by the Rutherford Appleton Laboratory to investigate the small pixel effect and charge sharing in 
spectroscopic CdTe and CdZnTe pixelated radiation detectors $[17,18]$. The PIXIE ASIC has dimensions of $3.7 \times 2.7 \mathrm{~mm}$ (see figure 3.5(a)) which can be bonded to a single X-ray detector fabricated from CdTe or CdZnTe. As shown in figure 3.5(b) the anode geometry of the resulting X-ray detector consists of four $3 \times 3$ pixel arrays with different pixel geometries (see table 3.1).

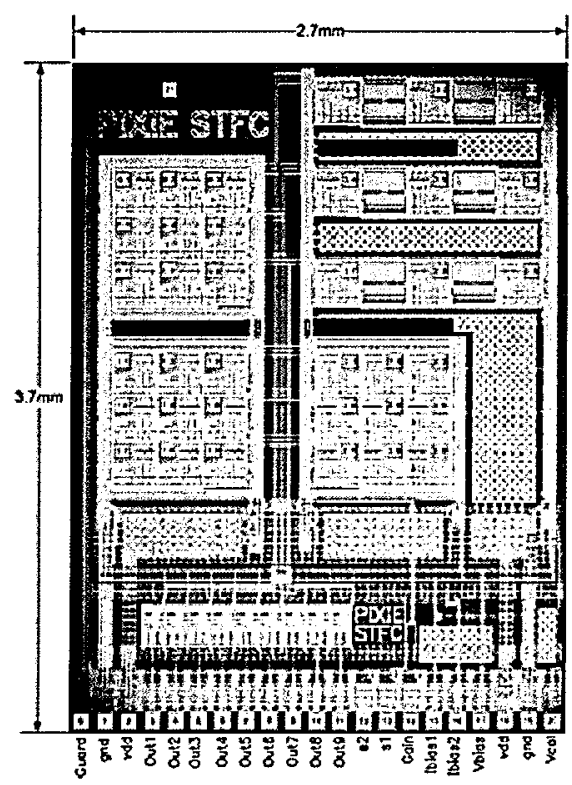

(a) PIXIE ASIC

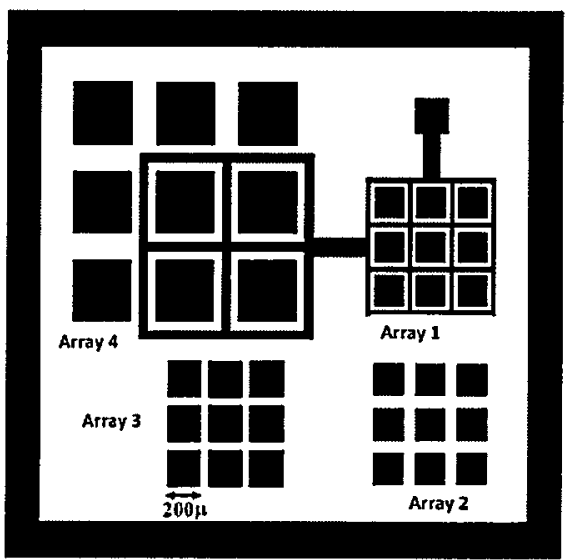

(b) Geometry of the pixel arrays

Figure 3.5: Picture of the PIXIE ASIC and the geometry of the pixel arrays for the PIXIE detectors used in this thesis.

\begin{tabular}{|c|c|c|c|c|}
\hline $\begin{array}{c}\text { Array } \\
\text { Number }\end{array}$ & $\begin{array}{c}\text { Pixel Pitch } \\
(\mu \mathrm{m})\end{array}$ & $\begin{array}{c}\text { Pixel Gap } \\
(\mu \mathrm{m})\end{array}$ & $\begin{array}{c}\text { Steering Grid } \\
(\text { Yes/No) }\end{array}$ & $\begin{array}{c}\text { Position in } \\
\text { Figure 3.5(b) }\end{array}$ \\
\hline 1 & 250 & 75 & Yes & Top Right \\
\hline 2 & 250 & 75 & No & Bottom Right \\
\hline 3 & 250 & 50 & No & Bottom Left \\
\hline 4 & 500 & 150 & Yes* & Top Left \\
\hline
\end{tabular}

Table 3.1: The dimensions of the four different PIXIE arrays shown in figure 3.5. * Array 4 only has steering grid round four pixels.

Figure 3.6 shows the active circuitry attributed to each pixel of the PIXIE ASIC. The circuitry consists of a non-shaping charge sensitive preamplifier and an output buffer which is then multiplexed directly off the ASIC. Each pixel also has a feedback circuit which provides detector leakage current immunity up to a value $250 \mathrm{pA}$ per 
pixel. Furthermore the central pixel of each array also contains a calibration circuit which has the ability to pass a pulser signal through the preamplifier of the pixel. This gives the capability to measure the noise performance of the PIXIE ASIC.

The PIXIE ASIC has two selectable gain modes which is selected by 1-bit digital input. The high gain mode is sensitive to incident X-ray energies up $150 \mathrm{keV}$, a low gain mode is also available which is sensitive to incident X-ray energies of up to 1.5 $\mathrm{MeV}$.

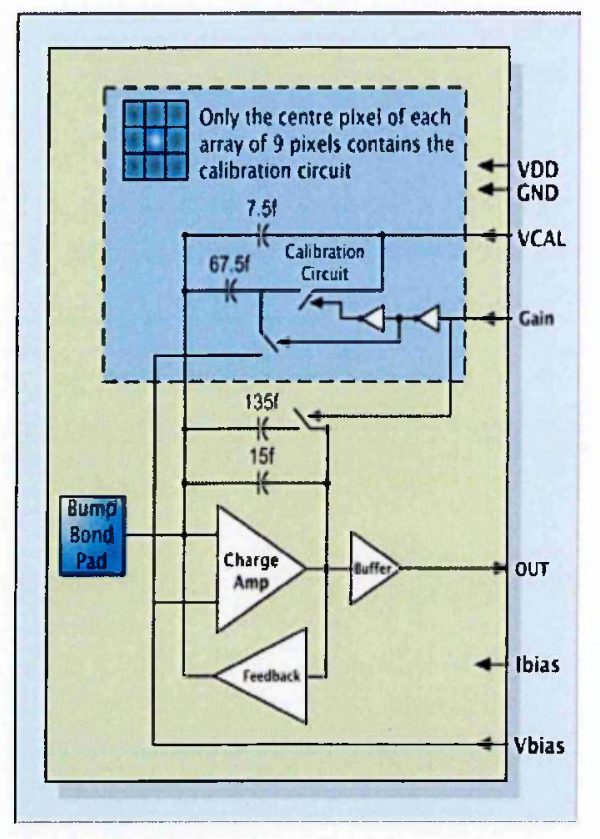

Figure 3.6: Block diagram of the individual pixel electronics.

The outputs of the nine pixels from the four arrays are multiplexed onto a 9-track analogue bus which are then driven off the chip by the output buffers. The array of interest is selected with a 2-bit digital input. The nine pixels of the selected array are then read off simultaneously allowing for detailed analysis of the pulse shapes generated by the pixel preamplifiers.

The use of the PIXIE ASIC has certain advantageous properties. The use of the ASIC makes it possible to investigate all the pulse waveforms gencrated by each pixel separately. Furthermore being able to mount the detector material directly to the PIXIE ASIC and reading off the raw preamplfier pulse shapes reduces the need 
for extra equipment which can result in an increase in the measured noise. Finally the PIXIE ASIC has four different pixels geometries. This means that a wide variety of pixel geometries can be investigated on the same detector material. As a result of this it removes the potential for variations in material properties from crystal to crystal.

To read out the signals generated by a $\mathrm{CdTe} / \mathrm{CdZnTe}$ detector mounted to the PIXIE ASIC a readout system was developed. A $23 \times 20 \times 11 \mathrm{~cm}$ diecast aluminium box was fitted with a variety of different sockets (see figure 3.7). The PIXIE ASIC is mounted to a custom made circuit board which has a variety of plugs and jumpers to control the PIXIE ASIC and to supply and receive the inputs and outputs from the detector. The board is screwed securely in place so to protect the PIXIE detector.

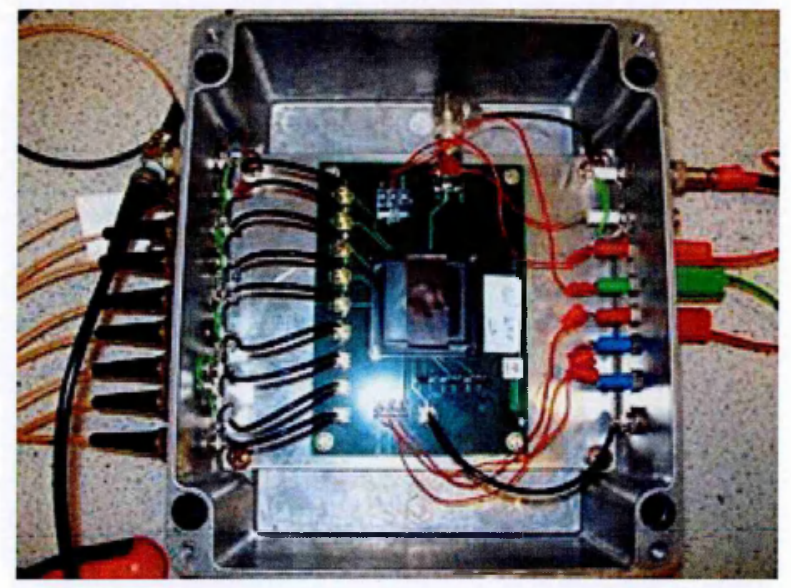

Figure 3.7: Photo of PIXIE ASIC readout system.

On the right side of the box shown in figure 3.7 there are a variety of sockets relating to the inputs to be supplied to the PIXIE ASIC and CdTe/CdZnTe detector. From top to bottom there are two male super high voltage (SHV) sockets which are used to supply bias to the detector and the interpixel steering grids. The detector bias is supplied to the detector via a short female Bayonet Neill Concelman (BNC) cable which plugs into an on board male BNC socket. Three, $4 \mathrm{~mm}$ panel sockets are used to supply $3.3 \mathrm{~V}$ to power the PIXIE ASIC, to connect the ASIC to ground and to supply $2.0 \mathrm{~V}$ to the pixel preamplifiers. Two further pancl sockets are used 
to measure the resistance across the onboard PT 100 thermistor which will give accurate values of the temperature of the system. Finally a BNC socket is used to supply test pulses to the central pixel calibration circuit. The output of the BNC socket is passed to the onboard male micro coaxial (MCX) socket via a flying MCX lead.

On the left hand side are nine BNC sockets which are used to receive the outputs from the nine pixels of the selected array. Flying MCX leads are used to transfer the outputs to the BNC socket which are then fed into the digital data acquisition system explained in the next section.

\subsection{XIA DGF-Pixie-4: Digital Data Acquisition System}

The preamplifier signal generated by the non-shaping charge sensitive preamplifier for each pixel can be processed in a variety of ways. One way to process and subsequently analyse the pulses is via the use of a digital data acquisition system (DAQ) like the XIA DGF-Pixie-4 Gamma Finder [45, 46].

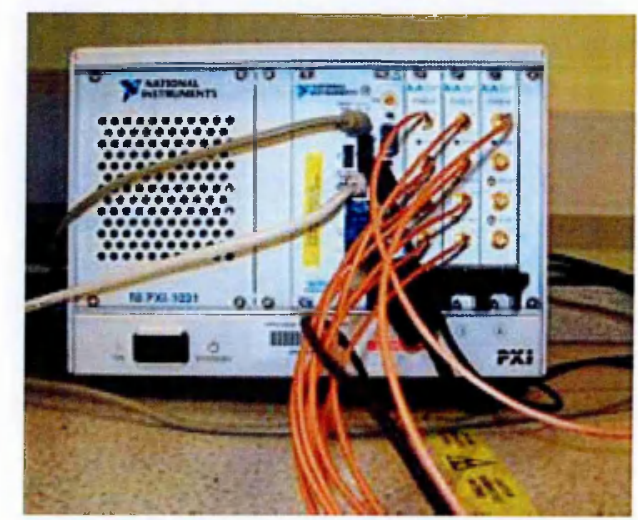

Figure 3.8: Photo of the XIA DGF-Pixie-4 system.

The Pixie-4 system consists of chassis (shown in figure 3.8) containing a compact PXI computer and three four channel Pixie-4 modules which are synchronized to share the same trigger. Initially the outputs from all nine pixels are fed into separate 
channels via BNC-SMA cables and then undergo the procedure shown in figure 3.9. Firstly the signals generated by the PIXIE ASIC are adjusted for gains and offsets, and then are digitized at a rate of $75 \mathrm{MHz}$ with a 14-bit Analogue-to-Digital Converter (ADC). After digital pulse triggering, pile-up inspection and a trapezoidal energy filter are implemented in a Field Programmable Gate Array (FPGA). The values relating to the trigger, pile-up inspection and the energy filter are inputted by the user via Igor pro software installed on the compact PXI computer.

If a valid event is detected a Digital Signal Processor (DSP) constructs a MultiChannel Analyser (MCA) spectrum from the pulse height of the trapezoidal filter. Furthermore it is possible for the user to record the raw digitized pulse shapes in a list mode run format. These raw pulse shapes can then be analysed offline with a bespoke piece made of LabVIEW software explained in section 3.5 .

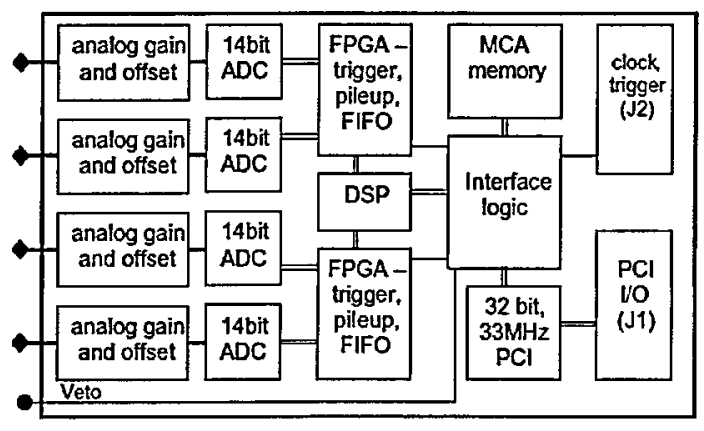

Figure 3.9: Block diagram of the XIA DGF-Pixie-4 spectrometer [46].

\subsection{LabVIEW Digital Pulse Processing Analysis Software}

A piece of LabVIEW software has been developed to analyse the pulse shape list mode file collected by the Pixie-4 system when a detector is mounted to the PIXIE ASIC and used in the system explained in section 3.3. The list mode file created by the DSP is saved and read into the LabVIEW software on an event by event basis the pulse shapes are read and analysed. For example figure 3.10 shows the 
pulses generated by the Pixie- 4 system for a $59.5 \mathrm{keV}$ gamma ray from ${ }^{241} \mathrm{Am}$ being collected by pixel 5 of a Redlen CdZnTe PIXIE detector.
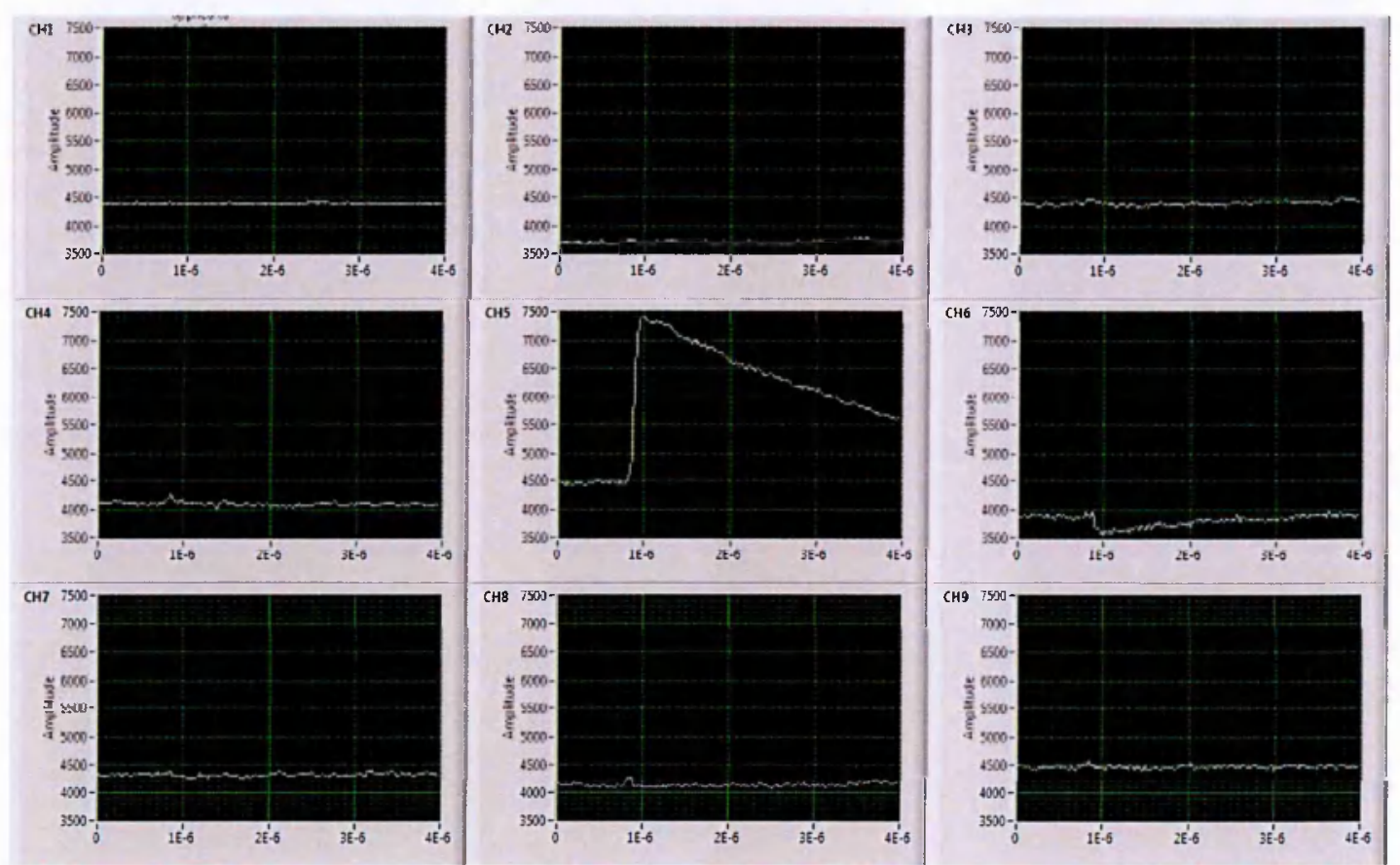

Figure 3.10: Pulses generated by the interaction of $60 \mathrm{keV}$ X-ray with pixel 5 of a Redlen CdZnTe detector. The pulses are recorded by the Pixie-4 DAQ system and processed with the LabVIEW software.

\subsubsection{CR-(RC) $)^{n}$ Digital Pulse Shaping}

When using electric circuits $\mathrm{RC}$ shaping refers to the use of resistor $(\mathrm{R})$ and capacitor (C) networks to alter a pulse shape. When using pulses created by a detector like the ones shown in figure 3.10 undergo shaping the orientation of the capacitor and resistor effects the final pulse shape. Furthermore the shaping has an effect on attenuating different parts of frequency noise. For both $\mathrm{CR}$ and $\mathrm{RC}$ networks the time constant $\tau$ is given by:

$$
\tau=R C
$$

where $R$ and $C$ are the resistance and capacitance of the electronic pulse shaping 
circuit.

A sketch of a CR differentiator network is shown in figure 3.11. The network is known as a differentiator network as when it is applied to an input voltage $\left(V_{i n}\right)$ and the time constant is sufficiently small the output voltage $V_{\text {out }}$ is the time differential of the input voltage as shown in equation 3.2. A CR network can also be known as a high pass filter. This is due to when the filter is applied to a pulse the low frequency noise is attenuated and not passed through the filter.

$$
V_{\text {out }} \cong \tau \frac{d V_{\text {in }}}{d t}
$$

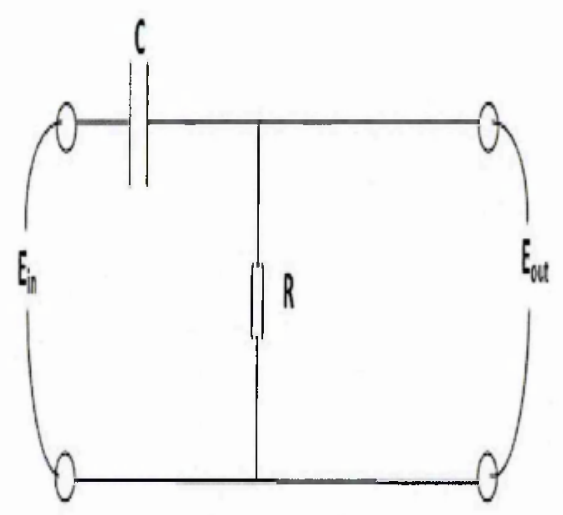

(a) CR network circuit

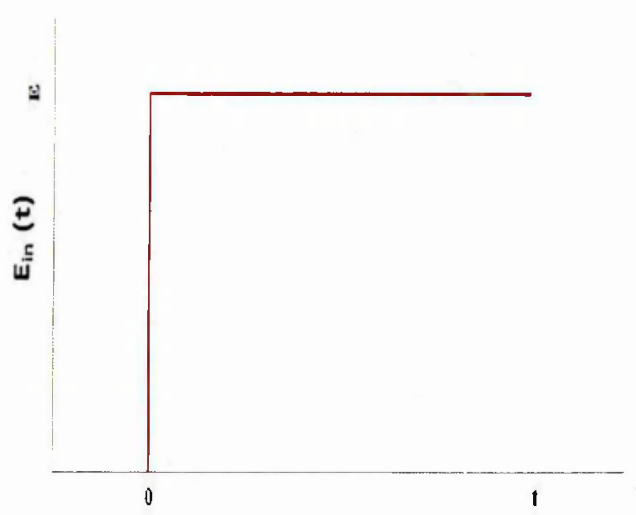

(b) Input step function

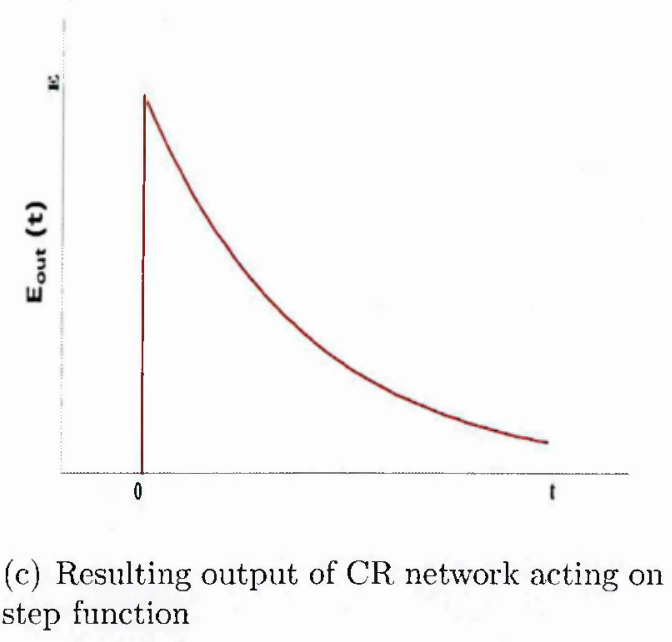

Figure 3.11: CR high pass filter or differentiator network and its effect on a step function input [3] 
Conversely a sketch of a $\mathrm{RC}$ network is shown in figure 3.12. The output of a pulse when entered into the circuit when the time constant is sufficiently small has the form shown in equation 3.3 hence the name of an integrator circuit. Furthermore when the circuit is applied to an input pulse the high frequency noise is filtered hence the alternate name of a low pass filter.

$$
V_{\text {out }} \cong \frac{1}{\tau} \int V_{\text {in }} d t
$$

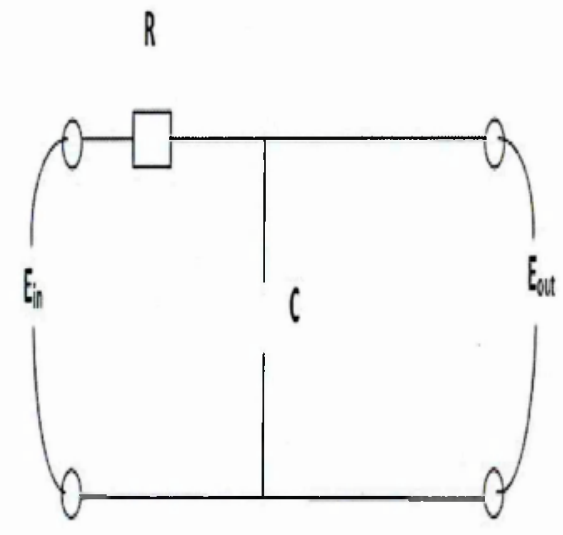

(a) RC network circuit

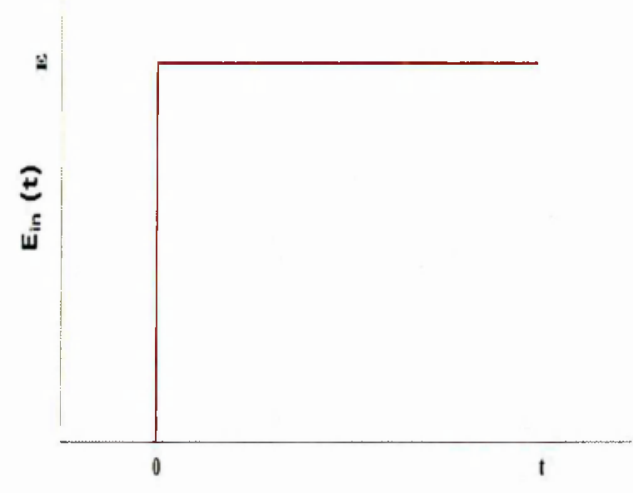

(b) Input step function

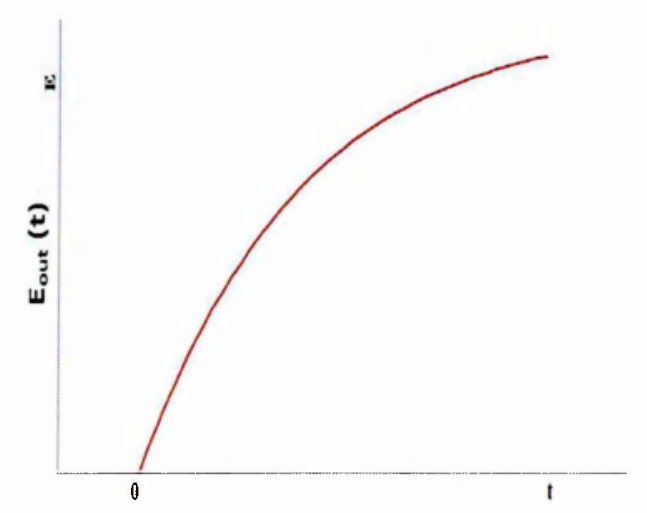

(c) Resulting output of $\mathrm{RC}$ network acting on step function

Figure 3.12: RC high pass filter or differentiator network and its effect on a step function input [3]

Both CR and RC networks can be applied digitally by performing numerical differentiation and integration on a pulse. The LabVIEW software has the ability 


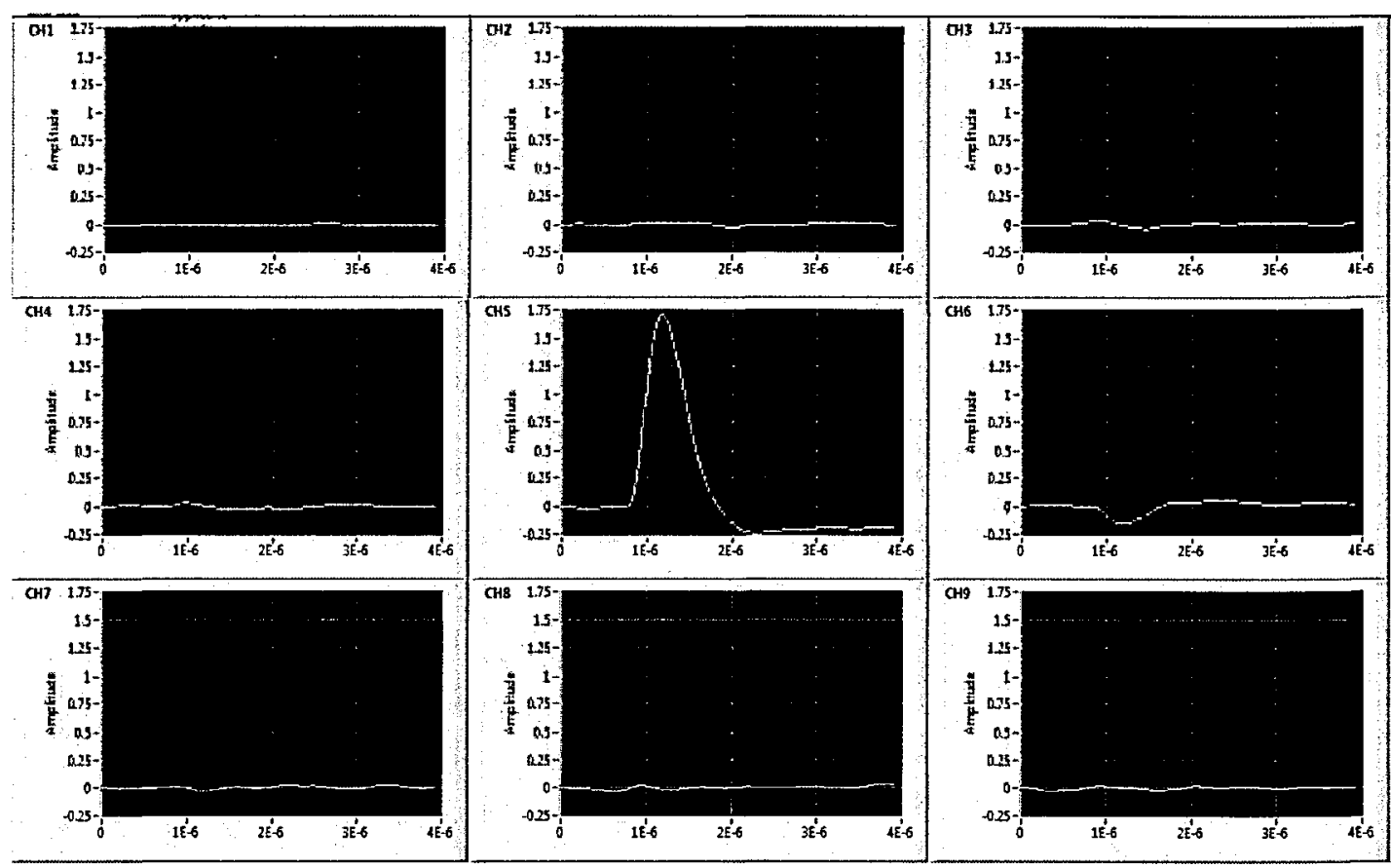

Figure 3.13: Output of CR-(RC) ${ }^{n}$ shaper when applied to the pulse shapes shown in figure 3.10 .

to perform a differentiation $\mathrm{CR}$ step and $n, \mathrm{RC}$ integration steps which results in the output voltage having the equation shown in equation 3.4. The resulting pulse shape is semi-Gaussian in nature and results in an increase in the signal-to-noise ratio as both low and high frequency noise has been filtered out. The parameters governing the CR-(RC) ${ }^{n}$ shaper are the number of steps of integration, $n$, and the time constant $\tau$. The values for the shaper are optimised to be $n=4$ and $\tau=0.5 \mu \mathrm{s}$ (see section 4.2.1.1 for the optimisation process). The resulting output of the shaper can be seen in figure 3.13, which is the output resulting from the digital shaping of the pulses shown in figure 3.10 .

$$
V_{\text {out }}=\frac{V_{\text {in }}}{n !}\left(\frac{t}{\tau}\right)^{n} e^{-t / \tau}
$$




\subsubsection{Pixel Spectra Generation}

Once all the pulses collected for a specific event have been CR-(RC) ${ }^{4}$ shaped it is possible to create a MCA spectrum for each pixel. On an event by event basis the amplitude $(A)$ of the shaped pulse is measured by the software. The software converts the amplitude to a channel number $(C h)$ by using equation 3.5 where Max $A$ and $N_{c h}$ are user defined values for the maximum amplitude of the shaper and the number of channels.

$$
C h=\left(\frac{A}{\operatorname{Max} A}\right) N_{c h}
$$

From this it is possible to create the spectrum collected by each pixel for a recorded data set. For example figure 3.14(a) shows spectra collected by each pixel of array 4 of a Redlen CdZnTe PIXIE detector when a irradiated by ${ }^{241} \mathrm{Am}$ source. It is possible to save the spectrum generated by each pixel in a text file to perform further analysis such as spectroscopy measurements (see section 4.2.1).

It is possible to convert the channel number generated by equation 3.5 to the energy collected by the pixel. This is achieved firstly by creating a calibration curve for each pixel. A plot of the recorded channel number against the incident X-ray energy results in a straight line. The resulting line of best fit can be used to convert the calculated channel number to collected energy in $\mathrm{keV}$ by using equation 3.6 where gain $(G)$ and offset $(O)$ are the gradient and y-intercept of the calibration curve respectively. Once the channel numbers have been converted to energy the single pixel spectrum can be plotted as function of energy as shown in figure 3.14(b).

$$
E=(G \cdot C h)+O
$$

\subsubsection{Event Classification and Sum Spectrum Generation}

The LabVIEW software measures the charge collected at each pixel in an event and covert this to a channel number. For each event if the calculated channel number for a pixel is above a user defined threshold it is classified as a hit, furthermore 

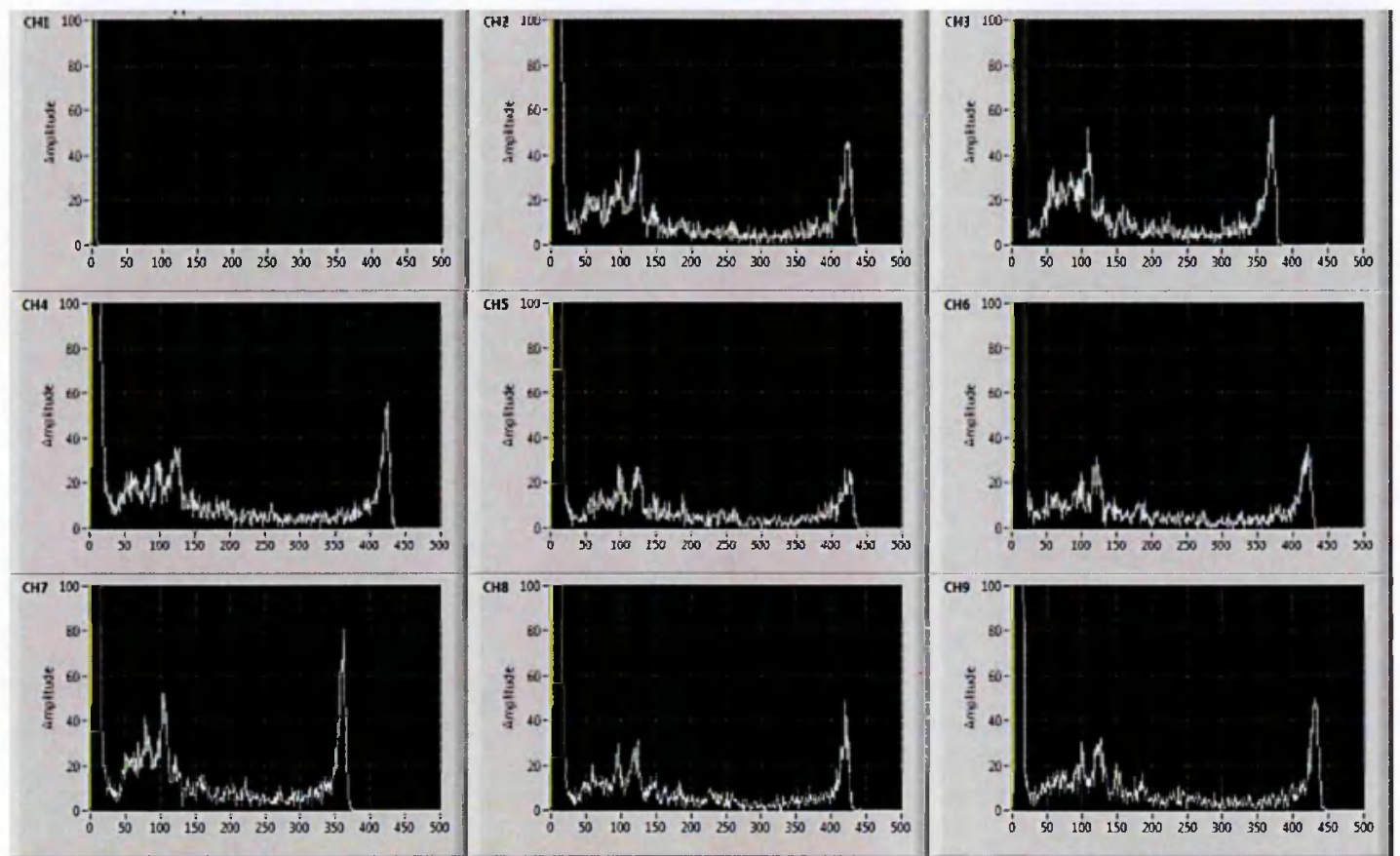

(a) Uncalibrated single pixel spectra
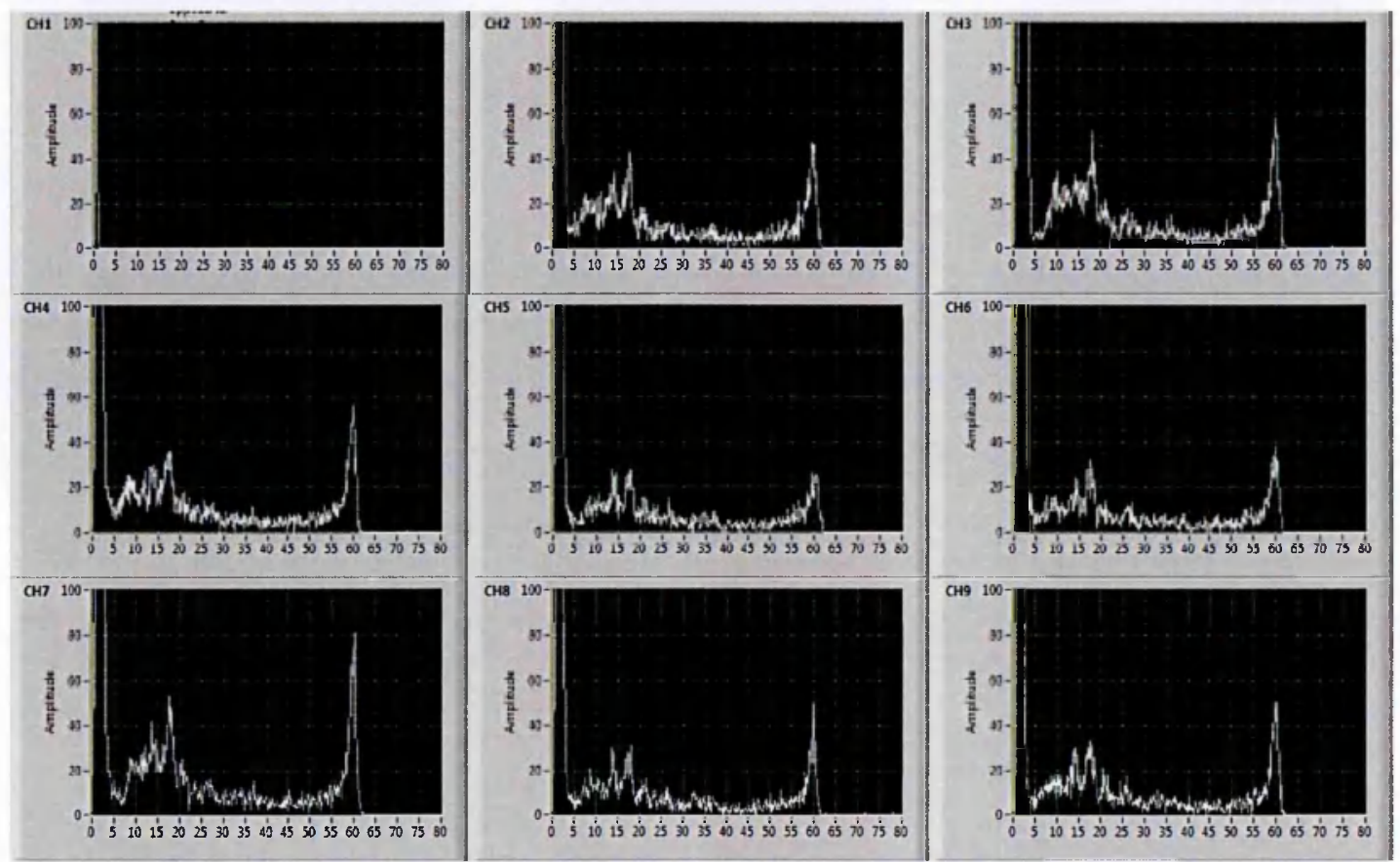

(b) Calibrated single pixel spectra

Figure 3.14: Spectrum recorded by each pixel when a Redlen CdZnTe PIXIE detector was irradiated by a ${ }^{241} \mathrm{Am}$ source. Spectra in (b) have been calibrated using cquation 3.6. It should be noted there is no recorded spectrum by pixel 1 as it is used to supply the bias to the guard ring of the detector. 
the number of hits per event is known as the event multiplicity $(m)$. The software outputs the hit pattern and multiplicity results for a set of events in two graphs shown in figure 3.15. The left hand graph is number of events recorded for each multiplicity value, the right hand graph is number of hits on each pixel for a data set. It is also possible to save the graphs in text file format for further analysis.

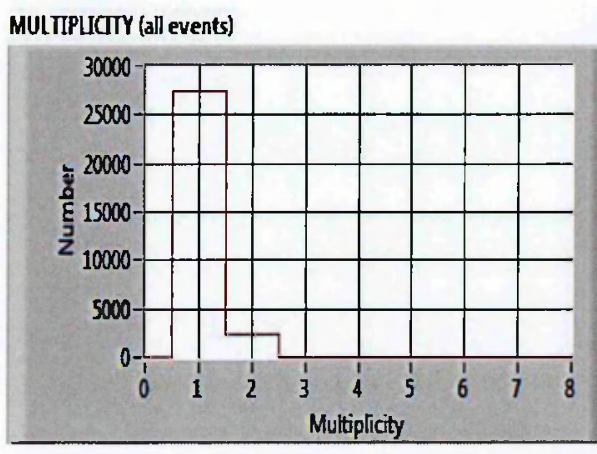

HIT PATTERN (after filter condition, and energy gate if applied)

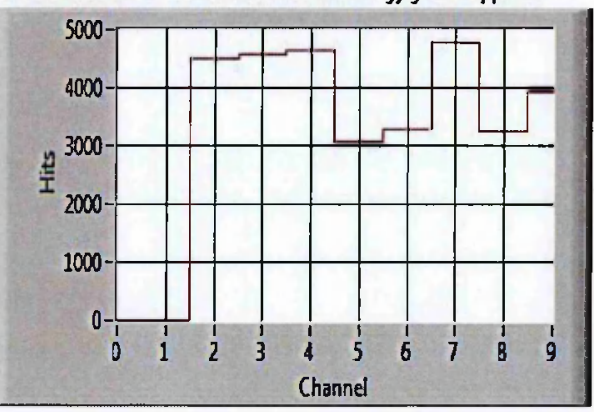

Figure 3.15: The multiplicity and hit pattern graphs generated by the LabVIEW software when array 4 of the Redlen PIXIE detector was irradiated by a ${ }^{241} \mathrm{Am}$ source.

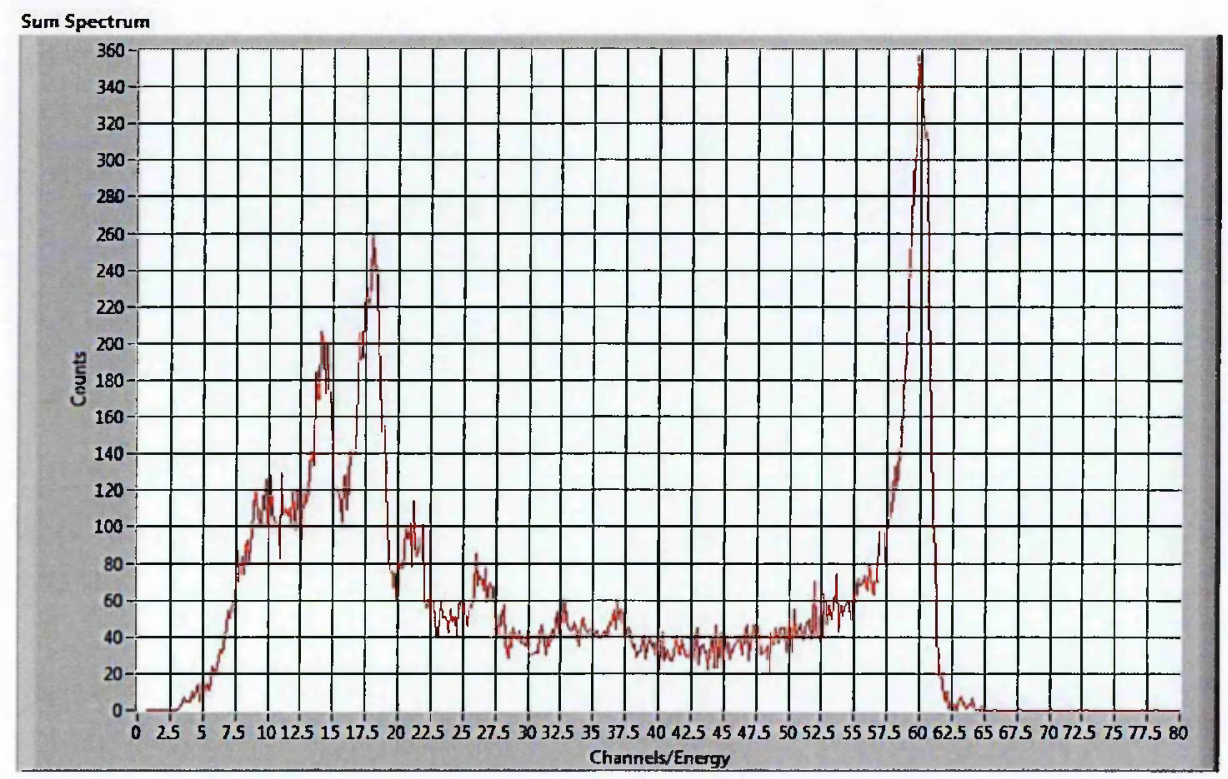

Figure 3.16: Sum spectrum generated by the LabVIEW software. Array 4 of the Redlen PIXIE detector was irradiated by a ${ }^{241} \mathrm{Am}$ source.

After classifying the multiplicity of each event it is possible to create a sum spectrum. 
For each event the sum of the calculated channel numbers for pixels which are classified as a hit are summed together. This results in the spectrum shown in figure 3.16. This means it is possible to compensate for charge sharing between adjacent pixels. Furthermore the software has the ability to gate on a specific region of the sum spectrum thus making it possible to calculate the hit pattern and multiplicity graphs at a specific energy.

\subsection{Focused X-ray Beamline Studies}

The I15 extreme conditions beamline at the Diamond Light Source was used to perform focused X-ray beam studies on a Redlen CdZnTe PIXIE detector. Line scans were performed between adjacent pixels so as to be able to investigate a wide range of effects.

Figure 3.17 shows a photo of the experimental setup used at the Diamond Light Source. The diecast aluminium box containing the Redlen PIXIE detector was attached to a motorized stage which could be moved in three dimensions using the software installed outside the target chamber. The focused X-ray beam entered the aluminium box through a Mylar window which meant it was possible to collect data with the lid of the box firmly attached without scattering the X-ray beam in the aluminium lid. The bias supplies for the detector and the bias's relating to the ASIC were based inside the target chamber. The Pixie-4 system was also based inside the target chamber which collected the data. Three meter monitor, keyboard and mouse extension cables were used so to make it possible to control the Pixie- 4 system outside the target chamber.

To find array 3 of the PIXIE detector the detector was irradiated with a $26 \mathrm{keV}$ $\mathrm{X}$-ray beam attenuated with $9 \mathrm{~cm} \mathrm{Al}$ and focused to a spot size of $2 \mathrm{~mm}$. The spot was moved in $50 \mu \mathrm{m}$ steps across where the array was thought to be and the counts/second recorded by pixel 5 (centre pixel) was recorded. Once the first rough approximation of where the array was completed three line scans was carried out with a $20 \mu \mathrm{m}$ spot to find the centre of pixel 5 (for more in depth analysis see section 


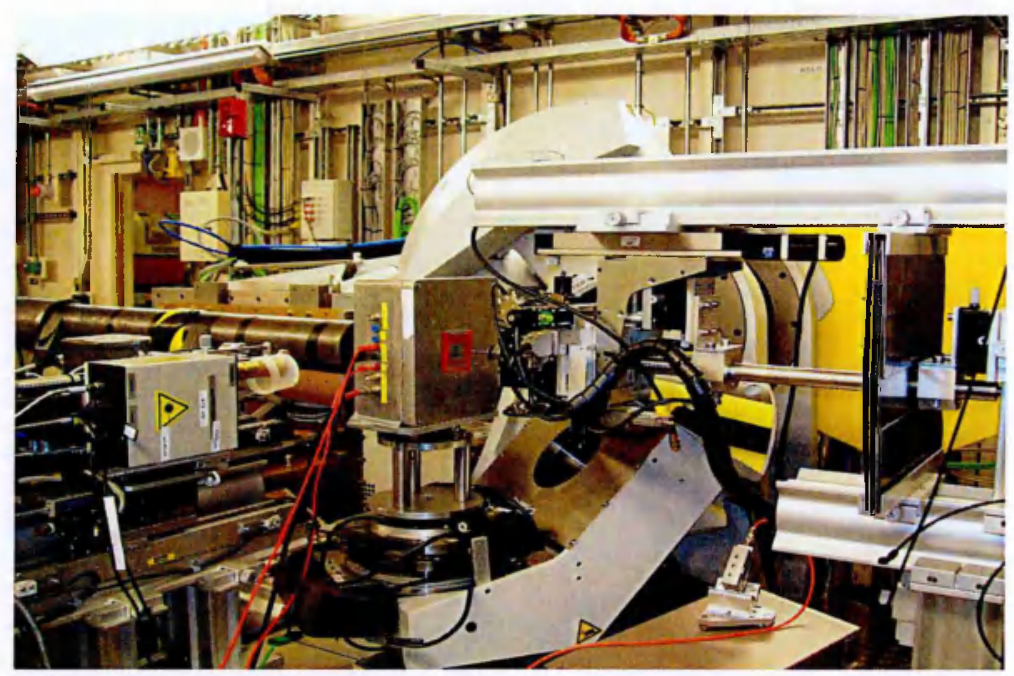

Figure 3.17: Photo of experimental setup used on the I15 beamline at the Diamond Light Source.

$5.1)$.

Once the centre of pixel 5 was found the detector was held at a bias of $-300 \mathrm{~V}$ and line scans were carried out between the centre of pixel 5 and 4 in $20 \mu \mathrm{m}$ steps with the X-ray beam collimated by a pinhole to a mean diameter of $20 \mu \mathrm{m}$. Xray beam energies of $26 \mathrm{keV}$ and $40 \mathrm{keV}$ were used to investigate how incident $\mathrm{X}$-ray energies above and below the $K$-shell absorption energies of $\mathrm{Cd}$ and Te effect the detector response. To protect the PIXIE ASIC and CdZnTe detector before collimation the $26 \mathrm{keV}$ and $40 \mathrm{keV}$ X-ray beams were attenuated with $1.5 \mathrm{~cm}$ and 5 $\mathrm{cm}$ aluminium respectively. This resulted in $99.95 \%$ of the X-ray being attenuated by the aluminium [4]. Further line scans were carried out at a X-ray beam energy of $40 \mathrm{keV}$ and detector biases of $-100 \mathrm{~V}$ and $-300 \mathrm{~V}$ to see the effect detector bias has on detector response. At each point on the line scans list mode files were created which were analysed offline with the dedicated LabVIEW software.

Example of a spectrum collected when the $26 \mathrm{keV}$ beam was incident on the centre of pixel 5 is shown in figure 3.18 . It clearly shows the $26 \mathrm{keV}$ photopeak and a series of further peaks. These are the result of the interaction of the third and fourth harmonics of the beam line with the detector material. These peaks were also used to investigate properties of the Redlen CdZnTe pixelated radiation detector. 


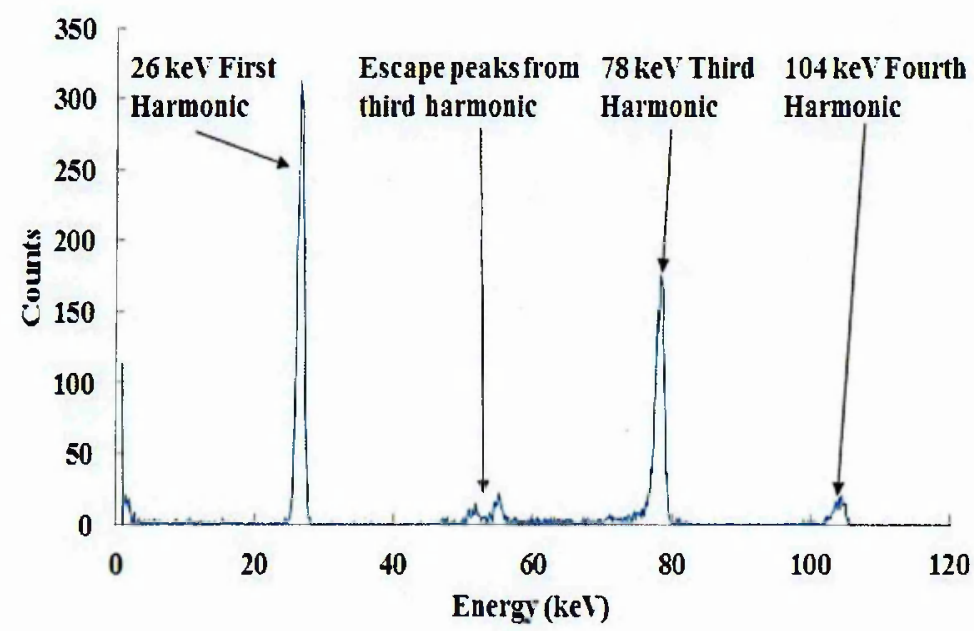

Figure 3.18: Spectrum recorded by pixel 5 when the $26 \mathrm{keV}$ beam was incident on the centre of the pixel. Spectrum clearly shows the $26 \mathrm{keV}$ photopeak and the detection of $78 \mathrm{keV}$ and $104 \mathrm{keV}$ third and fourth harmonics of the beam. No second harmonic is ibserved due to the properties of the monochromator.

For the complete analysis of the data collected at the Diamond Light Source refer to section 5 of this thesis. 


\section{Chapter 4}

\section{CdZnTe Detector Metrology and Spectroscopy}

$\mathrm{CdZnTe}$ is an attractive alternative semiconductor radiation detector. Its large band gap energy and high $Z$ number are desirable properties. However its poor hole transport properties can have a significant effect on the performance of the detector. The use of a single charge carrier sensitive electrode design such as a pixelated anode can correct for the poor hole transport properties. This chapter will involve detailed discussions on a variety of effects relating to pixelated $\mathrm{CdZnTe}$ radiation detectors. As previously explained in section 3.1 a fabrication process must be undertaken to create gold pixels on a CdZnTe sample. Once the gold pixels have been fabricated and Indium bump bonding procedure must undertaken to connect the detector to an ASIC such as the PIXIE ASIC or the Medipix ASIC. It is therefore desirable to characterise the material properties and the gold pixels before any interesting effects can be investigated. Therefore a metrology study of a CdZnTe Medipix detector was carried out. A $2 \mathrm{~mm}$ thick Medipix detector was characterised which has an anode of a $128 \times 128$ pixel array with the pixels on a $110 \mu \mathrm{m}$ pitch. Investigations into the macroscopic curvature of the material was carried out with a non contact optical profiler (Zygo Newview 200). The optical profiler was also used to perform microscopic studies of the surface roughness of the CdZnTe sample and the fabricated gold pixels. A scanning electron microscope was also used to characterize the gold 
pixels to support the data from the Zygo.

The second section of this chapter is an extensive investigation into the properties of a Redlen CdZnTe detector mounted to the PIXIE ASIC. Multiple radiation sources were used to carry out an X-ray and $\gamma$-ray spectroscopy study of the detector. The LabVIEW software wrote to analyse data taken from the PIXIE chip was optimised. Then studies into single and multiple pixel effects in pixelated $\mathrm{CdZnTe}$ radiation detectors was undertaken.

\subsection{Metrology Studies of a CdZnTe Medipix De- tector}

A macroscopic and microscopic study of a $15 \times 15 \times 2 \mathrm{~mm}$ CdZnTe Medipix [47] detector fabricated by the University of Freiberg was undertaken at the surface characterisation laboratories at Rutherford Appleton Laboratory (RAL) and the Material Science Department at the University of Surrey. The aim of this study was to provide information on the detector so a successful Indium (In) bump bonding procedure [48] can be undertaken at RAL resulting in an experimental campaign at the Diamond Light Source. A study on the macroscopic curvature of the device was undertaken which then developed into a study of the microscopic properties of the gold pixels and the surface roughness of the detector material.

\subsubsection{Curvature of CdZnTe material}

The macroscopic curvature of the device is of interest for the In bump bonding procedure. To bond the detector to the Medipix III ASIC $16 \mu \mathrm{m}-20 \mu \mathrm{m}$ diameter In bumps are fabricated on to the ASIC which are then used to create an electronic connection between the ASIC and the gold pixels fabricated to the detector material. The curvature of the device is therefore important as if it is too large some pixels may not connect to ASIC resulting in dead areas of the detector.

To measure the curvature a non-contact optical profiler called a Zygo was used (see 


\begin{tabular}{|c|c|}
\hline Line Scan & Height of Curvature $(\mu \mathrm{m})$ \\
\hline Diagonal & $\begin{array}{l}\text { Red Profile }=16.41 \\
\text { Blue Profile }=18.51\end{array}$ \\
\hline Vertical & 8.77 \\
\hline Horizontal & 6.31 \\
\hline
\end{tabular}

Table 4.1: Height of curvature for the surface profiles shown in figures 4.2-4.4.

section 3.2.1). Due to the large surface area of the detector the Zygo was run in a stitching mode resulting in 20 data sets being recorded and stitched together. Figure 4.1 is an oblique plot of the resulting from the stitching of the data sets. It clearly shows curvature across the detector material. To quantify this curvature line scans were carried out diagonally, vertically and horizontally through the centre of the detector material (see figures 4.2-4.4).

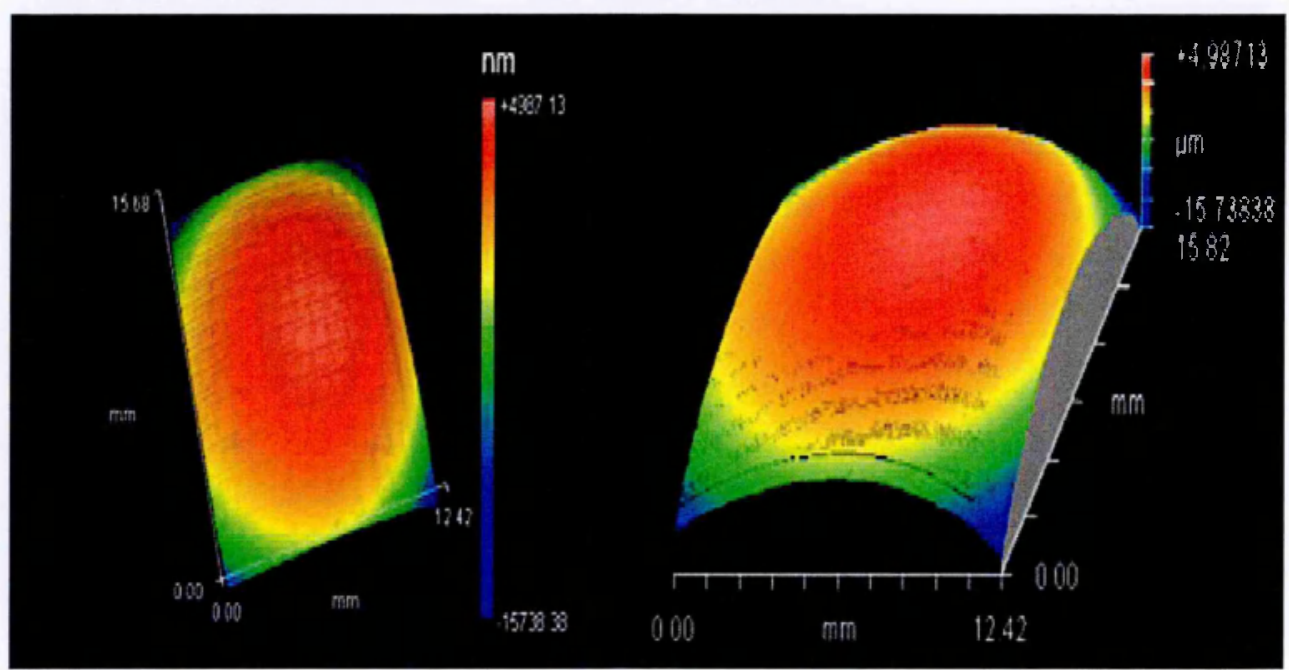

Figure 4.1: Macroscopic curvature of the CdZnTe Medipix detector.

Table 4.1 shows difference in the maximum and minimum height for the line scans shown in figures 4.2-4.4. There is a large amount of curvature compared to the typical curvature of an ASIC like a Medipix ASIC $( \pm 1 \mu \mathrm{m})$ [49]. This large amount of curvature will have a significant effect on the successful bonding of the detector to the Medipix ASIC. As previously explained the typical height of an In bump fabricated to a pixel of the Medipix ASIC is of the order of $16-20 \mu \mathrm{m}$. Therefore the significant curvature could result in pixels being lost in the corners of the device resulting in dead areas of the detector. 


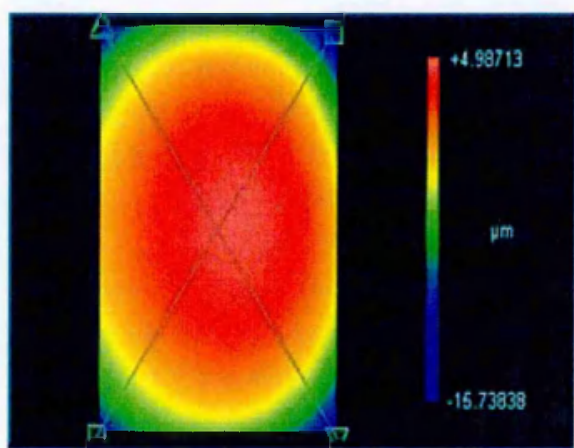

(a)

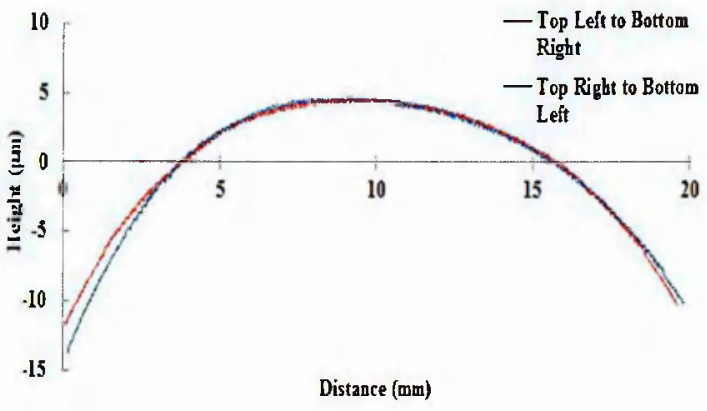

(b)

Figure 4.2: Diagonal line scan through centre of sample. The red and blue profiles are top left to bottom right corner and top right to bottom left corner respectively.

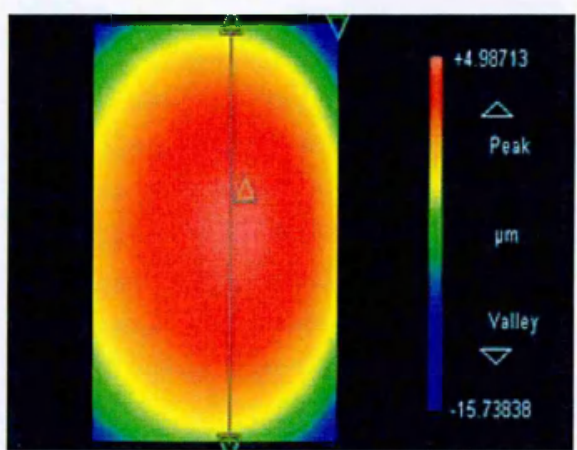

(a)

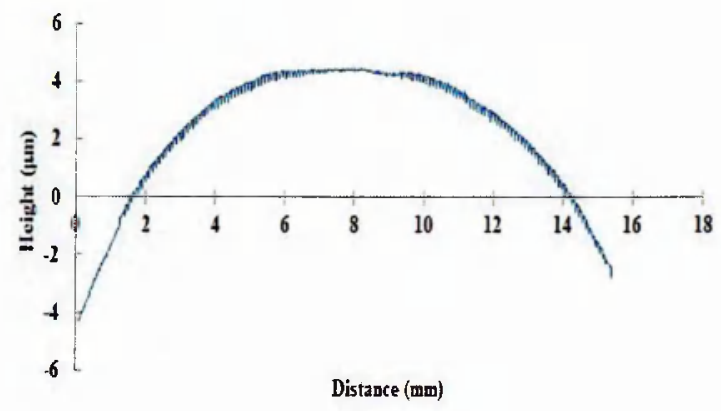

(b)

Figure 4.3: Vertical line scan through centre of the sample.

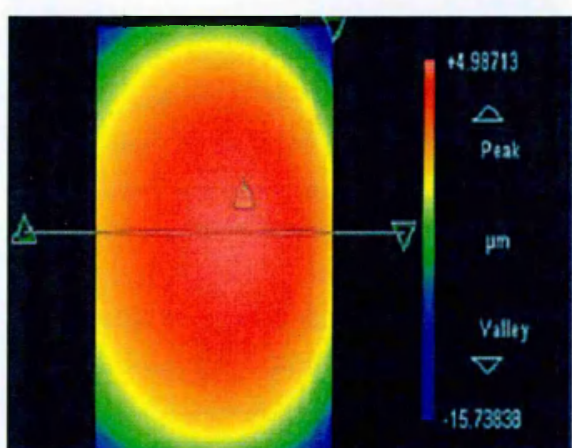

(a)

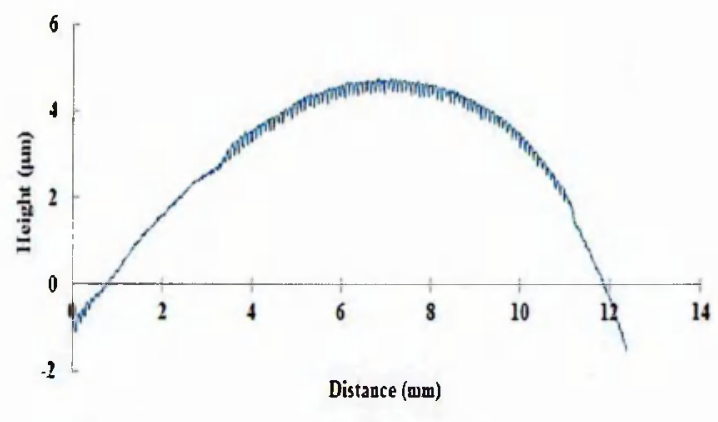

(b)

Figure 4.4: Horizontal line scan through centre of the sample. 


\subsubsection{Surface Roughness of the Bare CdZnTe Material}

The average roughness of the bare CdZnTe was also measured. A line scan was taken in the interpixel region resulting in the profile shown in figure 4.5. The surface roughness of the device is of interest as it may have an effect on the performance of the contact between the ASIC and the detector material. Furthermore the contact between the CdZnTe material and the gold pixel may be effected by the surface roughness.

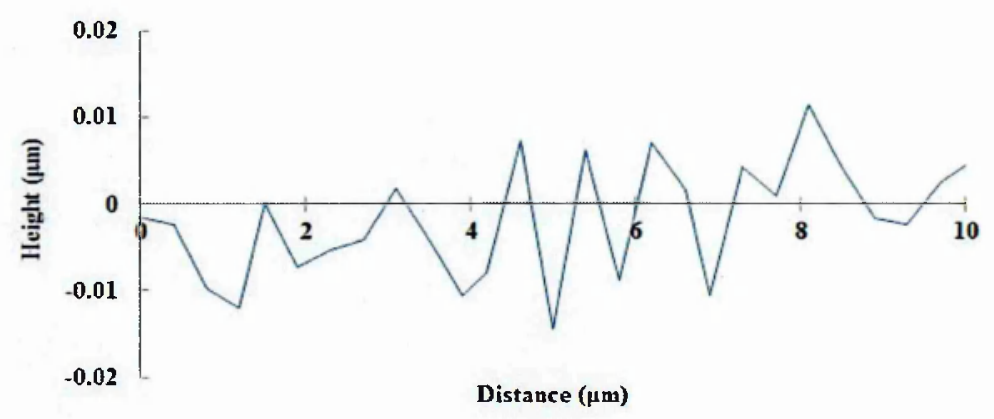

Figure 4.5: Profile of bare CdZnTe material with a calculated $R_{a}$ value of $6.56 \mathrm{~nm}$.

A common parameter to quantify the surface roughness of a matcrial is the arithmetic average deviation from the mean line of the profile which is known as the $R_{a}$ value. Equation 4.1 can be used to calculate $R_{a}$ value where $y_{i}$ is the vertical distance from the mean line to the $i^{\text {th }}$ data point and $n$ is the number of points on the line scan. The $R_{a}$ value of the profile shown in figure 4.5 is found to be $6.56 \mathrm{~nm}$.

$$
R_{a}=\frac{1}{n} \sum_{i=1}^{n}\left|y_{i}\right|
$$

The surface roughness of a gold pixel was also measured to quantify the effect the fabrication of gold pixels has on the recorded surface roughness. Figure 4.6 shows the measured surface profile of a gold pixel. The calculated $R_{a}$ value for the profile is $4.81 \mathrm{~nm}$ which is $1.75 \mathrm{~nm}$ less than the bare CdZnTe material. This surface roughness is sufficiently small that it will have little to no effect on the In bump bonding procedure. This is because the height of the In fabricated onto the CdZnTe is an order of magnitude bigger than the average roughness. 


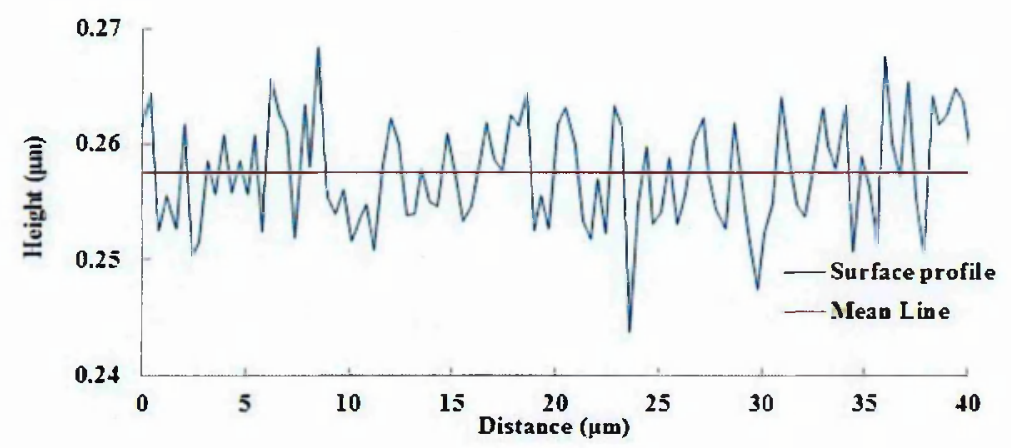

Figure 4.6: Profile of gold pixel with a calculated $R_{a}$ value of $4.81 \mathrm{~nm}$.

\subsubsection{Gold Pixel Characterisation}

A microscopic study of multiple single gold pixels fabricated to the CdZnTe was carried out with the Zygo in a high magnification mode. Figure 4.7 shows 3D plots of a variety of pixels and interpixel regions. The pixels were found to be $80 \times 80 \mu \mathrm{m}$ with a $30 \mu \mathrm{m}$ gap which was to be expected. It is found that the Zygo measured an increase in the gold pixel height on two of the edges of the pixels. The same two edges of the pisels showed the increased pixel height in this orientation the right hand and bottom edges showed increased height. To investigate this property a line scan was carried out horizontally across the pixel shown in figure 4.7(b).

Figure 4.8 shows the line scan across the pixel. The profile shows the gold pixel with a height of $260 \mathrm{~nm}$ and the increased gold height on the right hand side. The spike due to the increased gold is $154 \mathrm{~nm}$ higher than the pixel surface. To investigate whether the increased gold was an actual property of the detector or an artefact of the Zygo the sample was analysed using a Scanning Electron Microscope (SEM) (see section 3.2.2 for full description of equipment).

Figure 4.9 shows a SEM image of two different gold pixels. Figure 4.9(a) shows an example of a pixel where the pixel height is the same for all 4 edges. In comparison figure 4.9(b) shows in the bottom right hand corner a region where there is increased gold. To investigate this area the SEM was used in a higher magnification mode and focused on this corner.

Figure 4.10(a) shows a significant ridge where there is an increase in gold. There are regions round the ridges where there is shadowing as seen by the SEM due to 


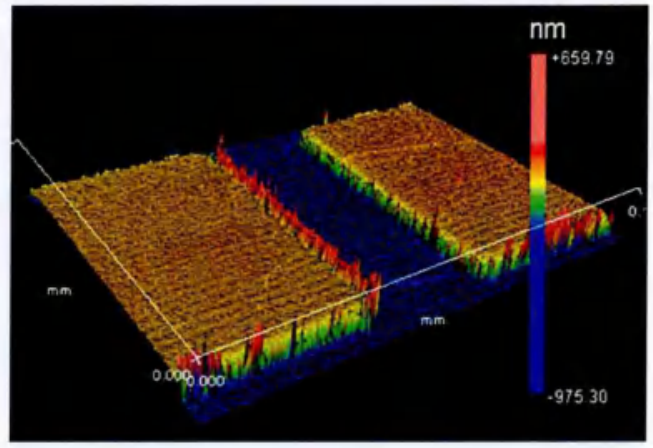

(a)

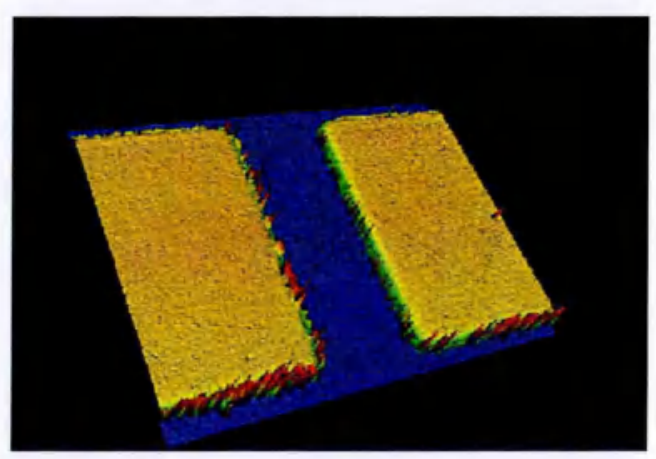

(c)

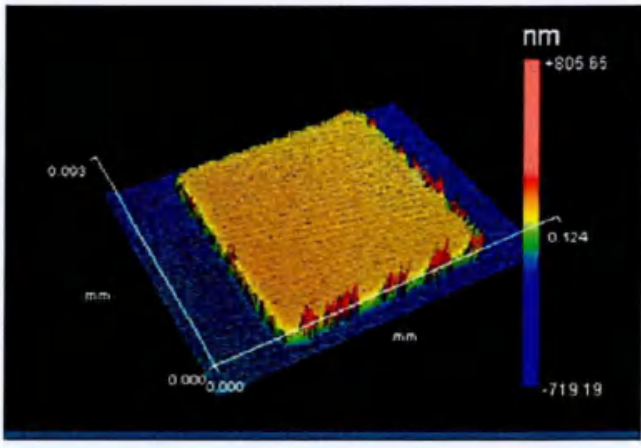

(b)

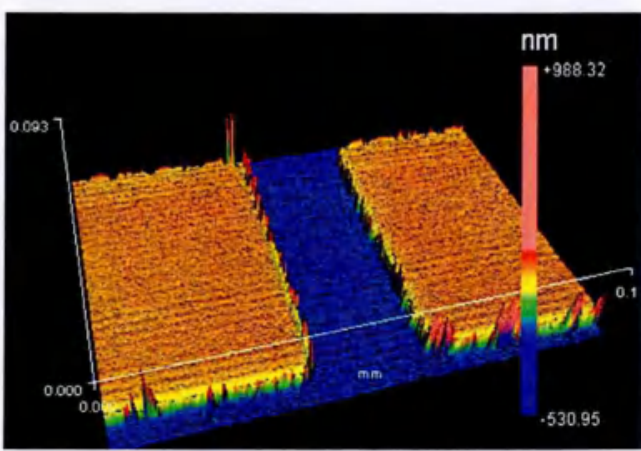

(d)

Figure 4.7: Example pixels from Medipix detector showing increase in gold on right and bottom sides.

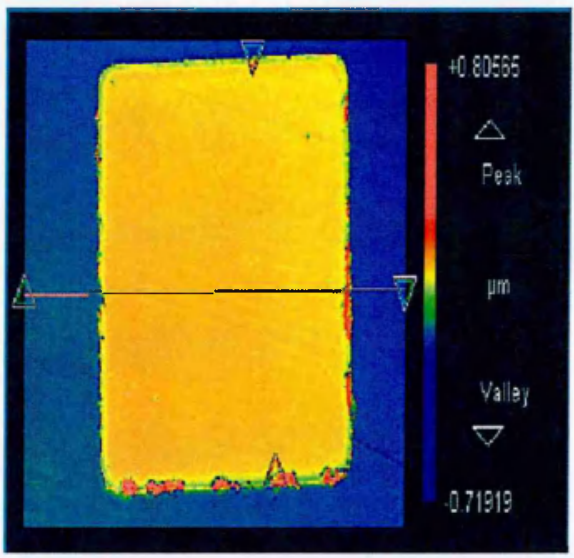

(a)

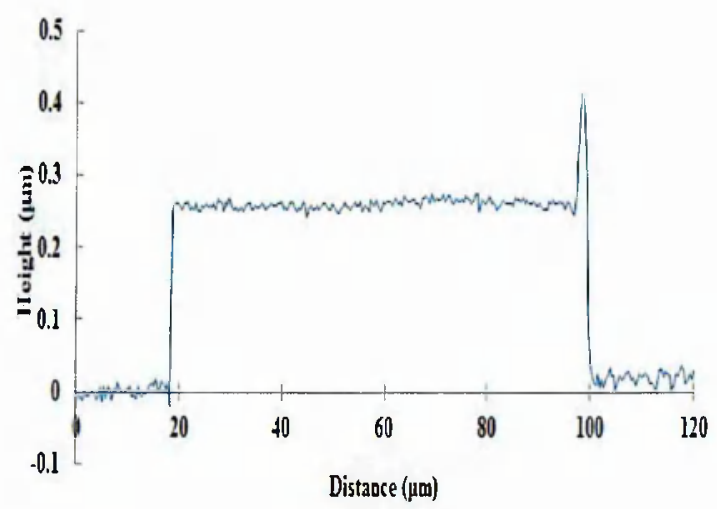

(b)

Figure 4.8: Pixel and resulting pixel profile showing the pixel height and the feature on the right side of the pixel. 


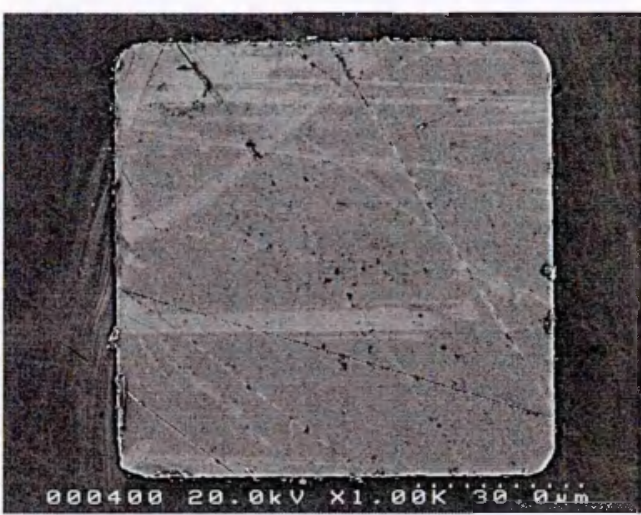

(a)

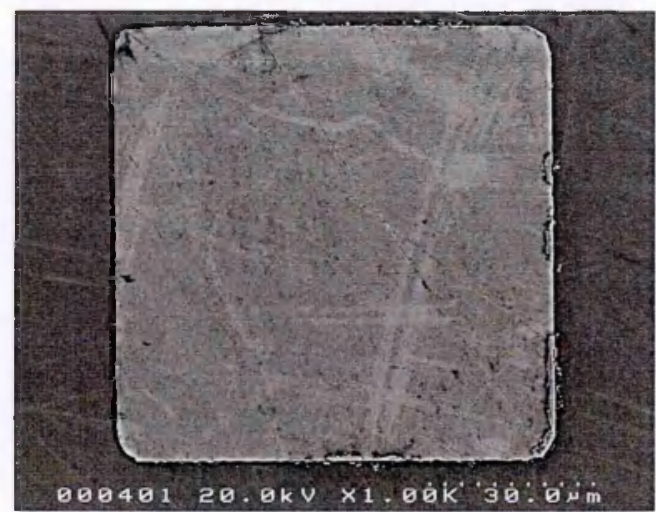

(b)

Figure 4.9: SEM image of two pixels to investigate gold profile on bottom and right edges.

the spike in the gold pixel height. In comparison figure 4.10(b) shows the difference between the edges. The top edge of the pixel has a clear and more defined edge compared to the right hand edge. It thought the origin of the gold pixel edge profiles is due to an issue with the lift-off process involved in the fabrication of the gold pixel contacts onto the CdZnTe material. As this detector was fabricated externally an in-depth knowledge of the specific photolithography process used by the external collaborator is not fully known.

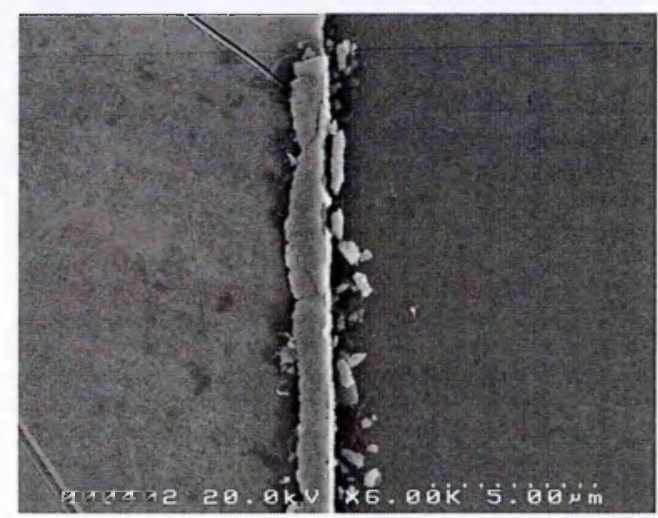

(a)

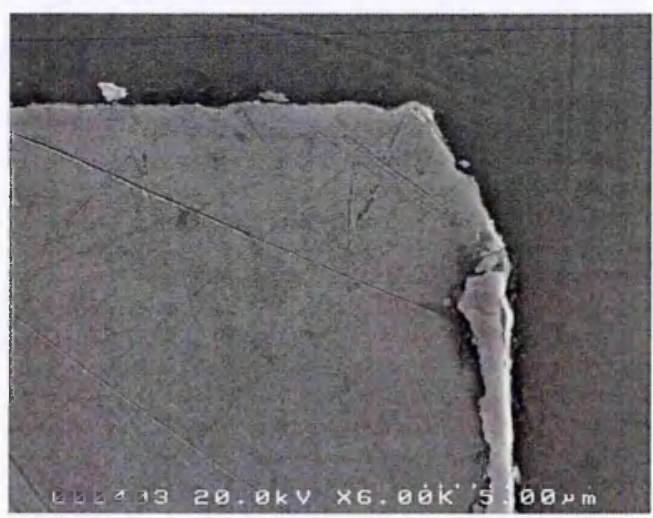

(b)

Figure 4.10: (a) SEM of the bottom right corner of figure 4.9(b) to show increased height of Gold contact. (b) SEM of the top right corner of figure 4.9(b) to show comparison between raised and un-raised edge. 


\subsection{Spectroscopy Studies of CdZnTe Redlen PIXIE}

\section{Detector}

An extensive study was carried out on a Redlen CdZnTe PIXIE detector so to be able to characterise and investigate a large number of effects relating to pixelated radiation detectors. Initially discussions into the optimisation of the LabVIEW data analysis software and the subsequent analysis of single pixel spectra generated by the PIXIE detector will be undertaken. The discussions will then be extended to multiple pixel spectra and charge sharing between adjacent pixels.

\subsubsection{Single Pixel Spectra}

As explained in section 3.5 a piece of LabVIEW software was developed to analyse the data collected from a PIXIE detector. One capability the software has is to generate the spectrum produced by each individual pixel. Single pixel spectra makes it possible to investigate detector properties such as the uniformity across an array, noise performance of a detector and how the detector bias effects the spectroscopic performance.

\begin{tabular}{|c|c|c|}
\hline $\begin{array}{c}\text { Array } \\
\text { Number }\end{array}$ & $\begin{array}{c}\text { Pixel Size } \\
(\mu \mathrm{m})\end{array}$ & $\begin{array}{c}\text { Pixel Gap } \\
(\mu \mathrm{m})\end{array}$ \\
\hline 1 & 175 & 75 \\
\hline 2 & 175 & 75 \\
\hline 3 & 200 & 50 \\
\hline 4 & 350 & 150 \\
\hline
\end{tabular}

Table 4.2: The dimensions of the four different PIXIE arrays.

Figure 4.11 shows the spectra collected by arrays 3 and 4 of the Redlen PIXIE detector (see table 4.2 for array dimensions) when a ${ }^{241} \mathrm{Am}$ source was incident on the detector material. The spectroscopic performance is clearly better for array 4 compared to array 3. As a result the $\mathrm{Np}$ characteristic X-rays are identifiable for spectra collected by array 4 but are not observable in the spectra collected by array 3. The increased spectroscopic performance is thought to be due to the decreased charge sharing between pixels. The effect on charge sharing on detector performance 

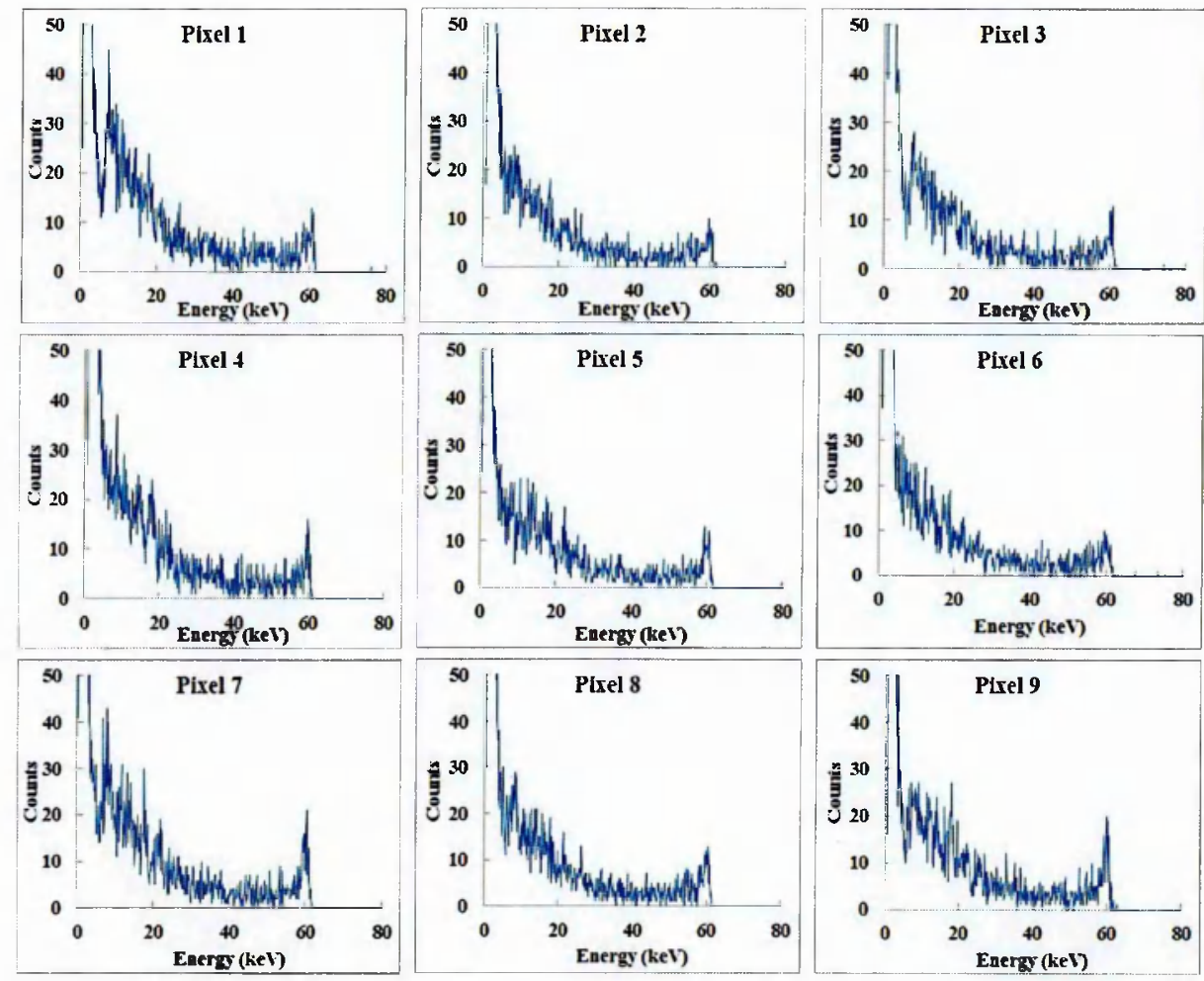

(a) Array 3
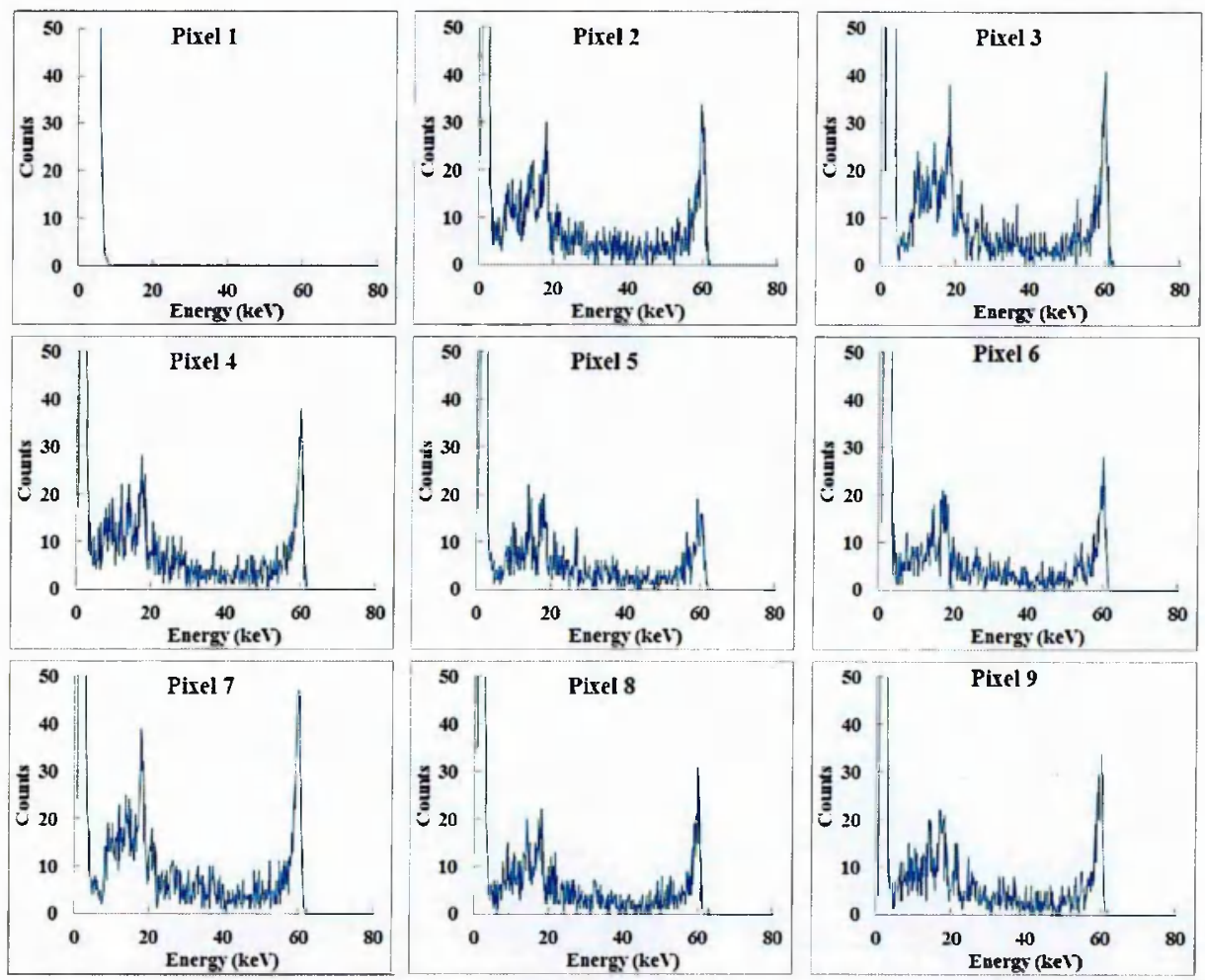

(b) Array 4

Figure 4.11: Single pixcl ${ }^{241} \mathrm{Am}$ spectra recorded by arrays 3 and 4 at a detector bias of $-300 \mathrm{~V}$. It should be noted that no spectrum should be recorded by array 4 pixel 1 as it is used to supply bias to the guard ring of the detector. 
will be investigated further in section 4.2.2.3. To investigate how certain parameters affect spectroscopic performance and to optimise parameters which operate the LabVIEW software the spectrum recorded by array 4 pixel 7 was used (see figure $4.12)$.

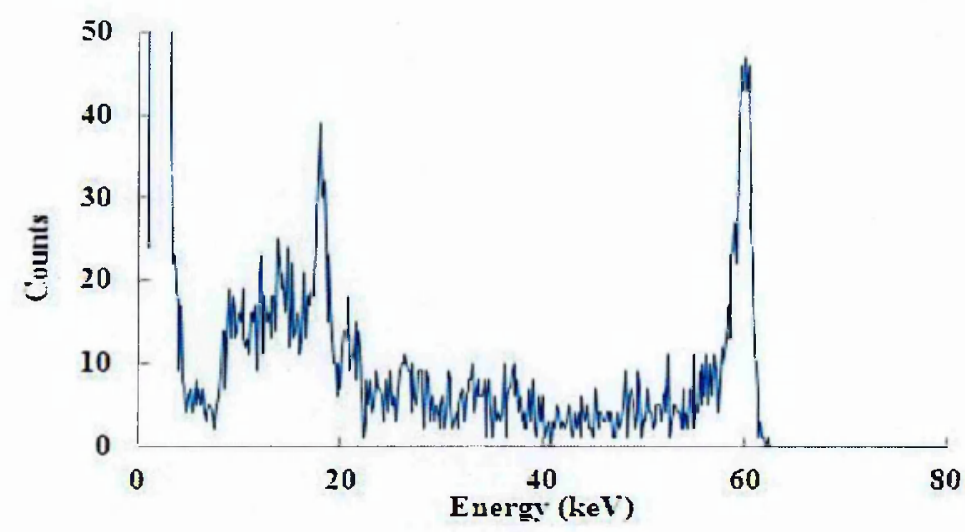

Figure 4.12: Single pixel ${ }^{241} \mathrm{Am}$ spectra recorded by array 4 pixel 7 at a detector bias of $-300 \mathrm{~V}$.

\subsubsection{Optimisation of LabVIEW Software Parameters}

Before any detailed analysis can be carried out on the detector performance and characteristics of the Redlen PIXIE detector certain parameters in the LabVIEW software need to be optimised. The LabVIEW software performs digital CR-(RC) ${ }^{n}$ shaping on the preamplifier pulses captured by the PIXIE-4 DAQ (see sections 3.4 3.5 for more details). Therefore the parameters used by the $\mathrm{CR}-(\mathrm{RC})^{n}$ digital shaper must be optimized. The two parameters of interest are the time constant, $\tau$ and the number of integration steps, $n$.

The first parameter optimised was the time constant of the shaper. The time constant effects the time over which the differentiation and integration steps of the shaper are carried out. To investigate how the time constant effects the recorded spectroscopic performance it was varicd between $0.3 \mu \mathrm{s}$ and $1 \mu \mathrm{s}$ in $0.1 \mu \mathrm{s}$ steps and the FWHM of the $59.5 \mathrm{kcV}{ }^{241} \mathrm{Am}$ peak was recorded.

It is found that the FWHM goes through a minima at a value of $0.5 \mu \mathrm{s}$. The form of this relation between FWHM and time constant is due to how the contribution 


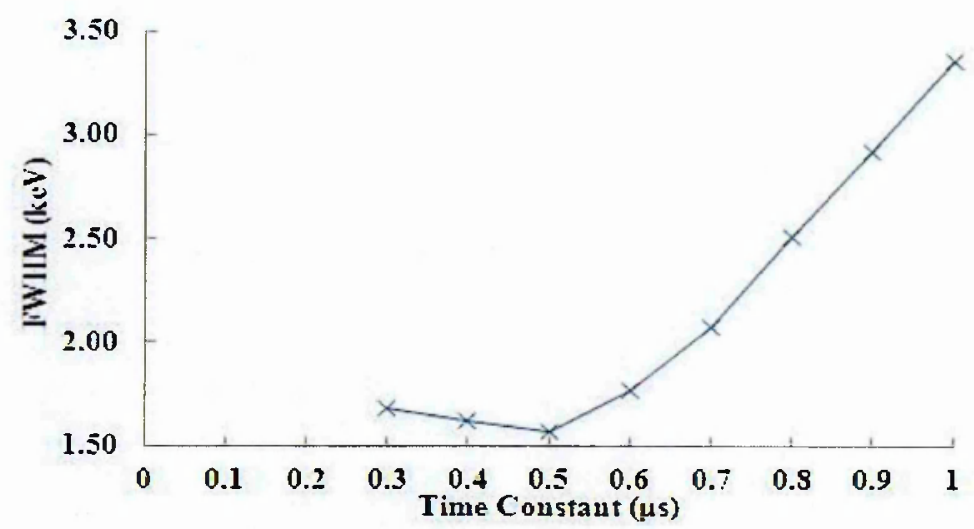

Figure 4.13: The effect of the CR-(RC) ${ }^{4}$ shaper time constant on the FWHM of the $59.5 \mathrm{keV}^{241} \mathrm{Am}$ peak at a detector bias of $-300 \mathrm{~V}$. It should be noted the leakage current at $-300 \mathrm{~V}$ is $-0.84 \mathrm{nA}$.

of specific types of electronic noise is effected by the time constant. Electronic noise is made up of three components:

- Series noise resulting from the input resistance of the PIXIE ASIC preamplifier.

- Parallel noise resulting from the leakage current of the detector.

- Flicker noise. Where the noise is proportional to $\frac{1}{f}$ where $f$ is the frequency of the electronic noise.

It is possible to characterise the amount of noise generated at a specific time constant by calculating the "equivalent noise charge" $(E N C)$. The $E N C$ is "the amount of charge that, if applied suddenly to the input terminals of a system, would give rise to an output voltage equal to the root mean squared level of the output due to only noise" [3]. Equations $4.2-4.4$ are the ENC equations for each of the three different noise components [50].

$$
E N C_{\text {series }}^{2}=b_{t} C_{d} k_{B} T r_{e q} \text { where } b_{t} \propto \frac{1}{\tau}
$$

$$
E N C_{\text {parallel }}^{2}=b_{s} i q \text { where } b_{s} \propto \tau
$$




$$
E N C_{\text {flicker }}^{2}=b_{f} C_{t o t} \frac{Q_{f}}{g m^{2}} \text { where } b_{f}=\text { constant }
$$

As shown in equations $4.2-4.4$ the amount of recorded series and parallel noise varies with the time constant $(\tau)$. At smaller values of $\tau$ the series noise dominates, as $\tau$ increase the amount of parallel noise increases and the series noise decreases. Furthermore the minimum recorded total noise will occur at a value of $\tau$ when the amount of series and parallel noise are equal. These equations thus explain the trend of how the FWHM varies with the time constant as shown in figure 4.13.

One source of electronic noise in a detector system is the leakage current from the detector. As shown in figure 4.14 as the detector bias increases the leakage current increases (-0.36 nA @-100 V compared to-0.84 nA@-300 V). The increase in leakage current at higher detector biases would result in a degradation in spectroscopic performance. However as shown in table 4.3 the charge transport properties of the detector improves at higher detector biases which tends to improve the spectroscopic performance. To investigate this further the time constant was varied for a ${ }^{241} \mathrm{Am}$ spectrum collected at $-100 \mathrm{~V}$ and compared to the data collected at $-300 \mathrm{~V}$ (see figure 4.15).

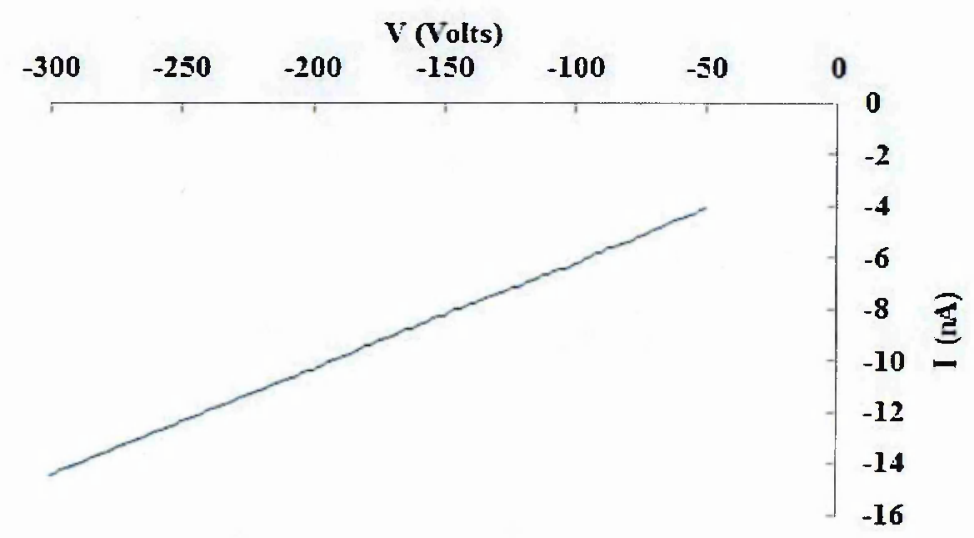

Figure 4.14: I-V characteristic of a single pixel to show how the leakage current increases with detector bias.

As shown in figure 4.15 the minimum value of FWHM is larger at a lower detector bias. This means the charge collection efficicncy is having a larger effect on the 


\begin{tabular}{|c|c|c|}
\hline $\begin{array}{c}\text { Detector Bias } \\
(\mathrm{V})\end{array}$ & $\begin{array}{c}\text { Leakage Current } \\
(\mathrm{nA})\end{array}$ & $\begin{array}{c}\text { Charge Collection Efficiency } \\
(\%)\end{array}$ \\
\hline-100 & -0.36 & 96 \\
\hline-300 & -0.84 & 100 \\
\hline
\end{tabular}

Table 4.3: The charge collection efficiency and leakage current measured at different detector biases.

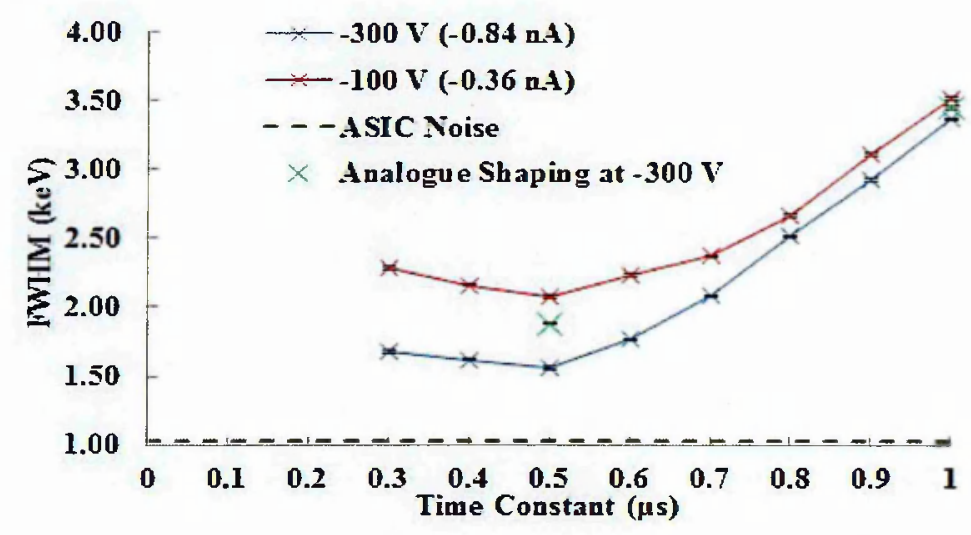

Figure 4.15: The effect of the CR- $(\mathrm{RC})^{4}$ shaper time constant on the FWHM of the $59.5 \mathrm{keV}{ }^{241} \mathrm{Am}$ peak at a detector bias of $-300 \mathrm{~V}$ and $-100 \mathrm{~V}$. The leakage current at each detector bias is also shown in the legend.

spectroscopic performance of the detector. As the drop in charge collection efficiency at lower detector biases tends to degrade the spectroscopic performance compared to the improvement in performance due to lower leakage current at lower detector biases.

The time constant of the $\mathrm{CR}-(\mathrm{RC})^{n}$ shaper is analogous to the shaping time of an analogue linear amplifier. Figure 4.15 also shows the FWHM of the $59.5 \mathrm{keV}$ peak at a detector bias of $-300 \mathrm{~V}$ and with analogue shaping at shaping times of $0.5 \mu$ s and $1 \mu \mathrm{s}$. As shown the FWHM is larger than the equivalent values of time constant. This shows that it is advantageous to analyse the spectra collected by Redlen PIXIE detector with the digital CR-(RC) ${ }^{n}$ shaper.

Figure 4.15 also shows the noise produced by the PIXIE ASIC. This noise was measured by applying a pulsed voltage supply to the calibration circuit of a PIXIE ASIC with no detector mounted to it. As a result the FWHM of the pulser peak produced is the noise due to the ASIC and the connecting cables. A value of 1.05 
$\mathrm{keV}$ was found which is minimum value for the electronic noise when a detector is mounted to the PIXIE ASIC.

The second parameter which operates the CR-(RC) ${ }^{n}$ shaper is the number of stages of integration $(n)$. To investigate how $n$ effects the spectroscopic performance the same ${ }^{241} \mathrm{Am}$ recorded at a detector bias of $-300 \mathrm{~V}$ was processed with values of $\mathrm{n}$ ranging from 1 to 8 with $\tau=0.5 \mu \mathrm{s}$. Figure 4.16 shows that the spectroscopic performance improves up to a value of $n=4$ where the $\mathrm{FWHM}=1.56 \mathrm{keV}$. At values $n>4$ the FWHM degrades.

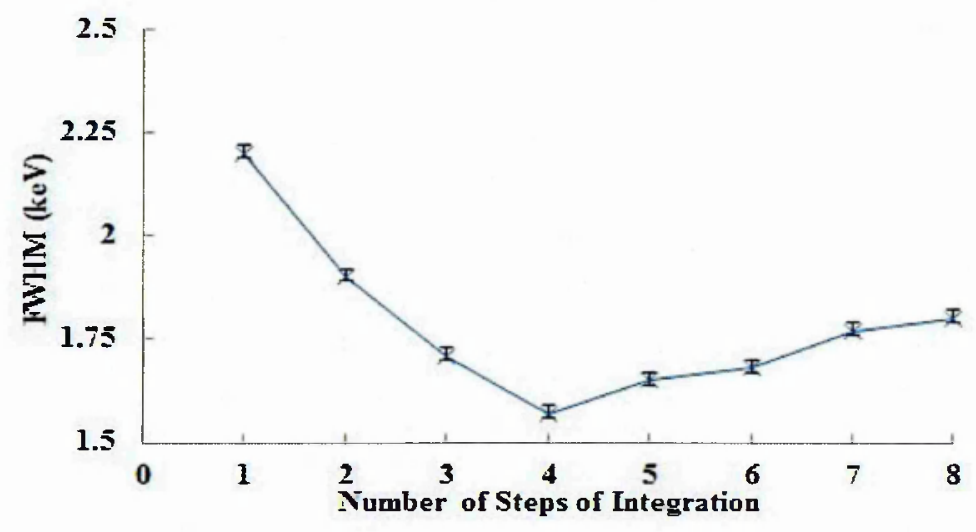

Figure 4.16: The effect number of steps of integration $(n)$ of the CR-(RC) ${ }^{n}$ shaper on the recorded detector performance.

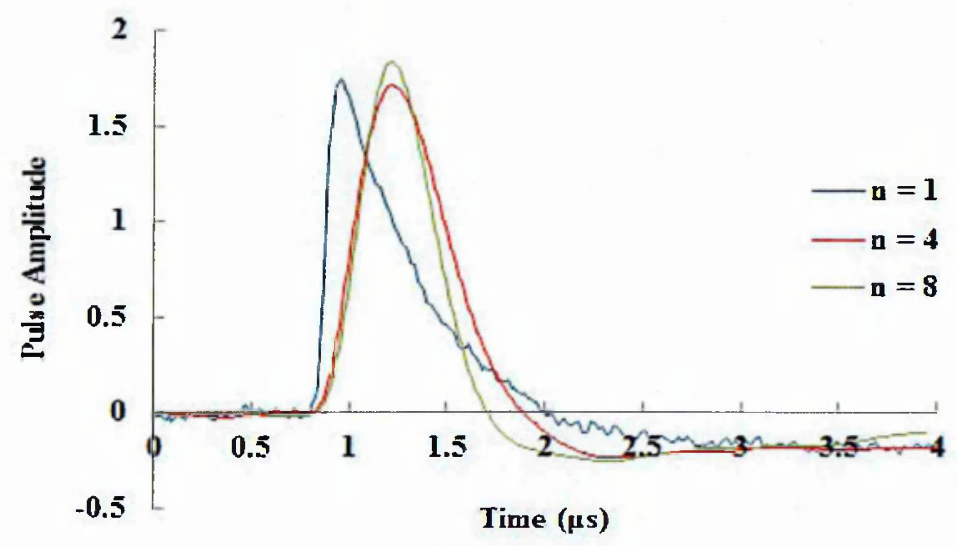

Figure 4.17: Resulting shaped pulses for different number of steps of integration $(n)$ for the CR-(RC) ${ }^{n}$ shaper with a fixed time constant of $0.5 \mu \mathrm{s}$.

The reason for the trend in the FWHM can be explained by the shape of the output 
of the CR-(RC) ${ }^{n}$. Figure 4.17 shows the resulting output of the shaper for the interaction of a $59.5 \mathrm{keV} \mathrm{X}$-ray with the detector material which is subsequently detected by pixel 7 of array 4 of the PIXIE detector. As $n$ increases the shape of the pulse becomes more Gaussian in nature upto a value of $n=4$. At values of $n>4$ there is less modification to the shaper output. The characteristics of the pulse can be quantified by calculating the signal-to-noise ratio of the pulse. The signal-to-noise ratio is calculated by the use of equation 4.5 , where $\mu$ is the expected value and $\sigma$ is standard deviation of the noise.

$$
\text { Signal-to-noise }=\frac{\mu}{\sigma}=\frac{\text { Amplitude of pulse }}{\sigma_{\text {noise }}}
$$

To calculate the signal-to-noise ratio at each value of $n$ the shaper output of pixel was recorded for 5 separate events of a $59.5 \mathrm{keV}$ X-ray interacting with the detector and being subsequently detected by the pixel. The average signal pulse amplitude and standard deviation of the noise was recorded and the signal-to-noise was calculated using equation 4.5. As shown in table 4.4 as $n$ increases the signal-to-noise ratio increases upto a value of $n=4$. The subsequent higher signal-to-noise ratio results in the trend of improving FWHM for values of $n$ upto a value of $n=4$.

\begin{tabular}{|c|c|}
\hline$n$ & signal-to-noise ratio \\
\hline 1 & $82.9 \pm 12.9$ \\
\hline 2 & $170.2 \pm 9.1$ \\
\hline 3 & $173.1 \pm 9.0$ \\
\hline 4 & $185.7 \pm 7.4$ \\
\hline 8 & $176.5 \pm 8.3$ \\
\hline
\end{tabular}

Table 4.4: Signal-to-noise ratio for different values of $n$.

Therefore in any subsequent analysis of spectra collected by a PIXIE detector values of $\tau=0.5 \mu$ s and $n=4$ will be used for the CR-(RC) ${ }^{n}$ shaper.

\subsubsection{Detector Bias Effect on Detector Performance}

As explained in the previous section the bias applied to the detector has a large effect on the recorded spectroscopic performance. To investigate this relationship 
more in depth, the detector bias was varied between $-100 \mathrm{~V}$ and $-300 \mathrm{~V}$ in $-50 \mathrm{~V}$ steps and the spectrum recorded by all 8 available pixels was recorded.

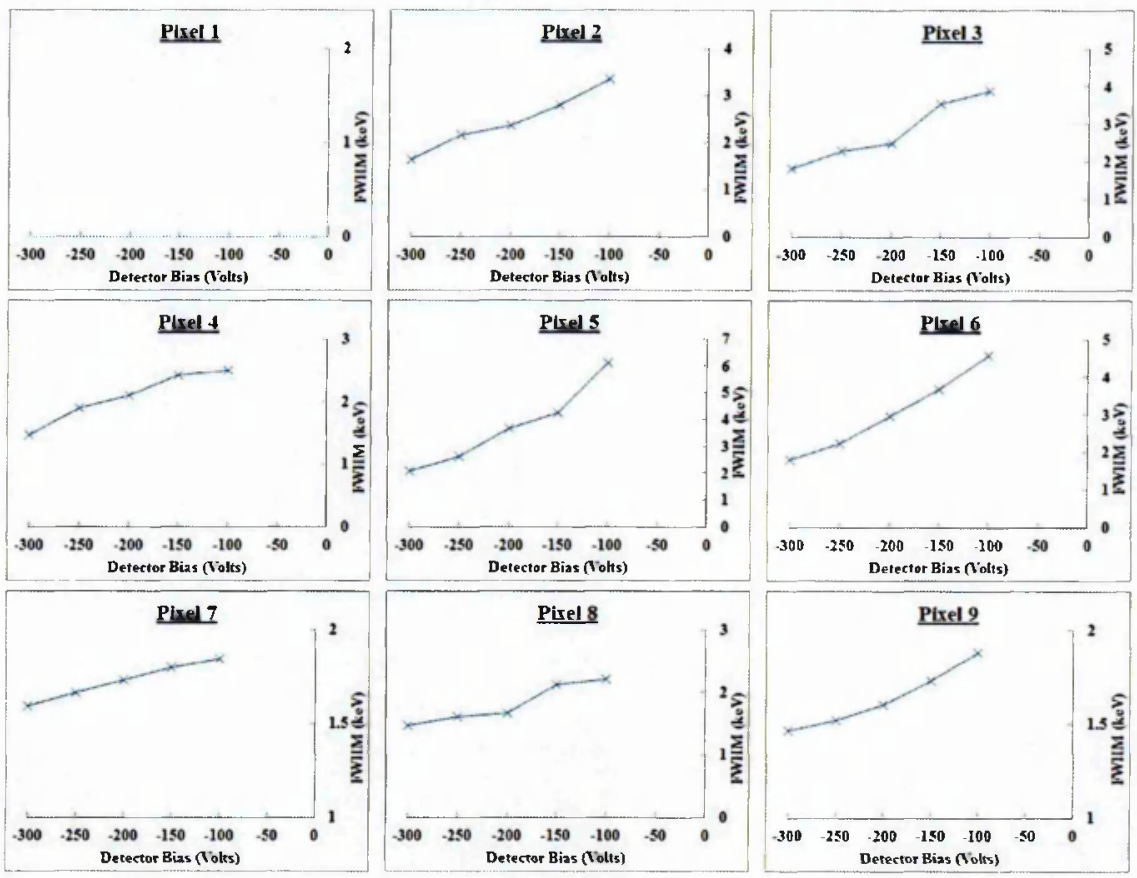

Figure 4.18: The effect of detector bias on the single pixel performance of array 4 of the Redlen PIXIE detector. As previously explained no data is collected from pixel 1 as supplies bias to the guard ring of the detector.

As explained in the previous section the leakage current and charge transport properties have a large effect on the recorded spectroscopic performance. It was found that at higher detector biases the leakage current increases degrading the detector performance. However as shown in the figure 4.19 the charge collection efficiency increases with detector biases which dominates the resulting FWHM.

$$
\mathrm{CCE}=\frac{\mu_{e} \tau_{e}(-V)}{d^{2}}\left[1-\exp \left(\frac{-d^{2}}{\mu_{e} \tau_{e}(-V)}\right)\right]
$$

Equation 4.6 is the Hecht equation which can be used to fit the charge collection efficiency graphs shown in figure 4.19 [51]. Where $\mu_{e} \tau_{e}$ it the mobility lifetime product of the electron in the material, $V$ is the detector bias and $d$ is the detector thickness.

The equation is formulated for data collected from detectors with two planar elec- 


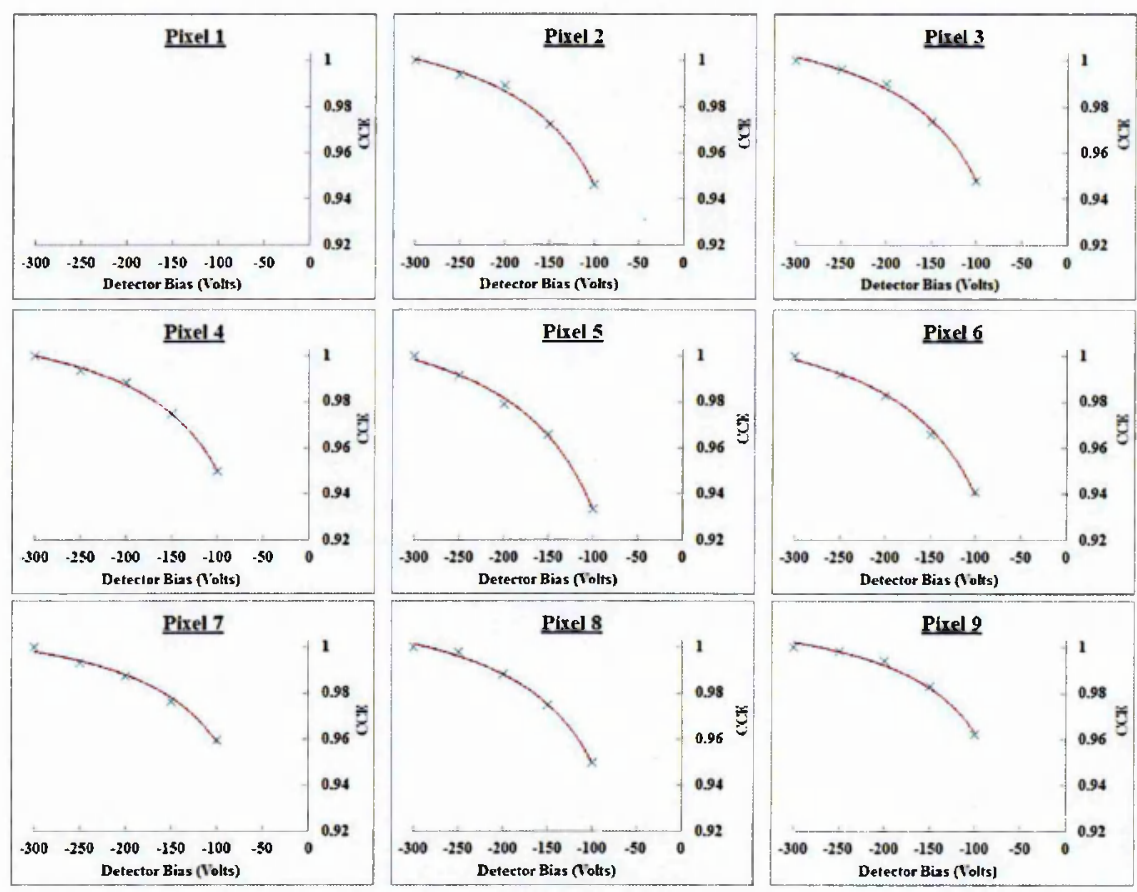

Figure 4.19: Charge Collection Efficiency (CCE) and the resulting Hecht equation fits to calculate the $(\mu \tau)_{e}$ for each pixel of array 4 .

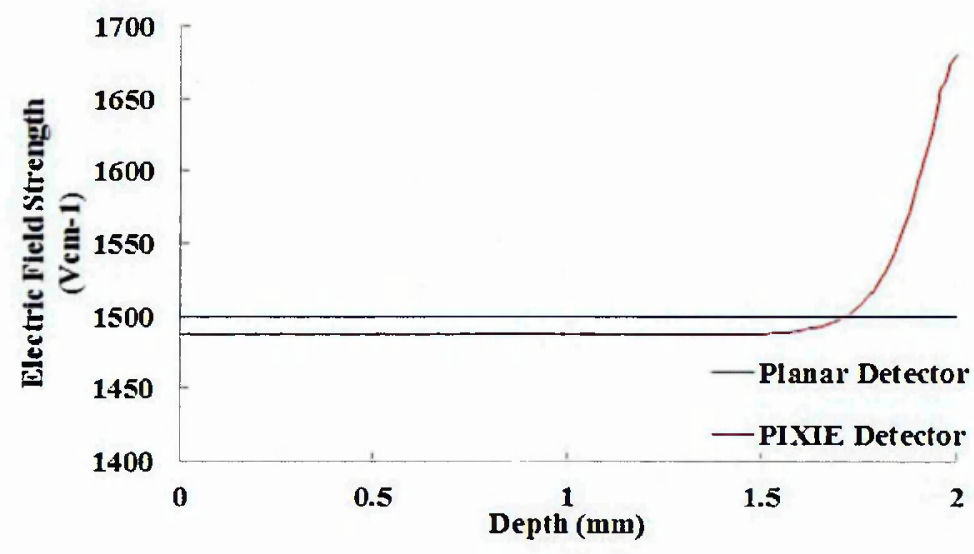

Figure 4.20: A TCAD simulation of the electric field profile across the width of a planar and PIXIE detector at a detector bias of $-300 \mathrm{~V}$. Where $0 \mathrm{~mm}$ is the cathode electrode and $2 \mathrm{~mm}$ is the centre of a pixel anode electrode [52]. 
trodes which have a different electric field profile compared to pixelated detectors. Figure 4.20 shows a TCAD [53] simulation of the electric field profile for a planar detector and from the centre of a pixel for a simulated array 4 of a PIXIE detector [52]. As shown the electric field profiles are the same across the majority of the device. It is found that the field profiles only differ dramatically at depth positions near the pixelated anode. Therefore for a basic analysis of the charge transport properties of the detector the Hecht equation was fitted to the data and the $(\mu \tau)_{e}$ values were extracted (see table 4.5 ).

\begin{tabular}{|c|c|}
\hline Pixel & $(\mu \tau)_{e}\left(\mathrm{~cm}^{2} \mathrm{~V}^{-1}\right)$ \\
\hline 1 & $\mathrm{~N} / \mathrm{A}$ \\
\hline 2 & $2.3 \times 10^{-3}$ \\
\hline 3 & $2.4 \times 10^{-3}$ \\
\hline 4 & $2.6 \times 10^{-3}$ \\
\hline 5 & $1.9 \times 10^{-3}$ \\
\hline 6 & $2.2 \times 10^{-3}$ \\
\hline 7 & $3.3 \times 10^{-3}$ \\
\hline 8 & $2.5 \times 10^{-3}$ \\
\hline 9 & $3.3 \times 10^{-3}$ \\
\hline
\end{tabular}

Table 4.5: Mobility Lifetime product $(\mu \tau)_{e}$ for each pixel of array 4 calculated from Hecht equation fits to data shown in figure 4.19

As shown in figure 4.21 there is good correlation between pixels with the best FWHM at $-300 \mathrm{~V}$ and the ones with the highest $(\mu \tau)_{e}$. Therefore the charge transport properties of the material for each specific pixel has a large effect on the spectroscopic performance of a pixel. 


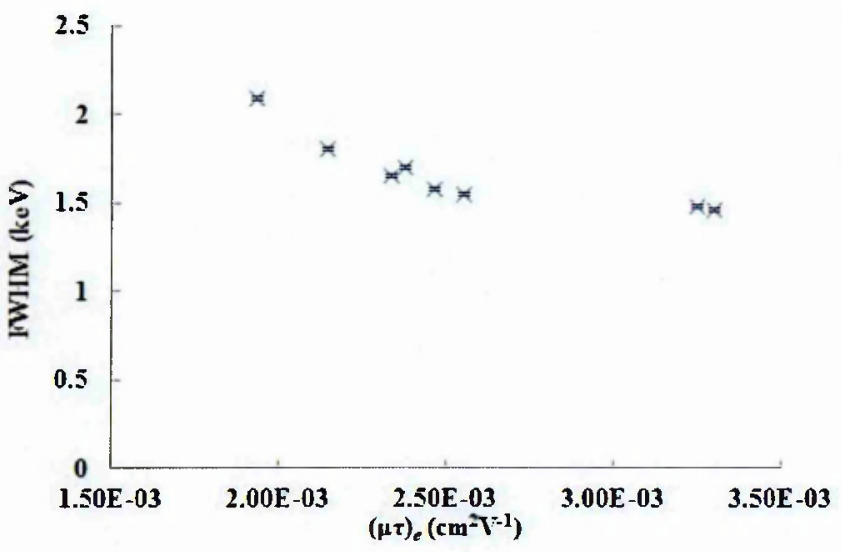

Figure 4.21: FWHM of a single pixel spectrum as function of the $(\mu \tau)_{e}$ value for that pixel.

\subsubsection{Sum Spectra and Multiple Pixel Effects}

The LabVIEW software has the ability to investigate multiple pixel effects in pixelated radiation detectors. These can involve the generation of sum spectra and the phenomena of charge sharing between pixels. This section will investigate these effects.

\subsubsection{Gain Matching of the Detector}

Before any detailed analysis involving multiple pixel effects can be carried out a pixel gain matching procedure must be undertaken. It is possible that each pixel in an array will have different gains and offsets. As shown in figure 4.22 and table 4.6 the differences in the gains and offsets result that for the same energy measured by different pixels the channel number generated by the CR-(RC) ${ }^{4}$ shaper is different. The discrepancy in gains and offsets can be due to slight differences in the pixel preamplifier gains for the PIXIE ASIC. Furthermore the material properties over the array may vary for example the difference in the $(\mu \tau)_{e}$ for each pixel as shown in table 4.5. Figure 4.23 shows the shows the peak centroid channel of the 59.5 $\mathrm{keV}$ peak for each pixel of array 4 as a function of the $(\mu \tau)_{e}$ for that pixel. It is found that the peak centroid is approximately constant for all $(\mu \tau)_{e}$ values except for pixels 3 and 7 . This means that the main effect on the difference in gains is duc 

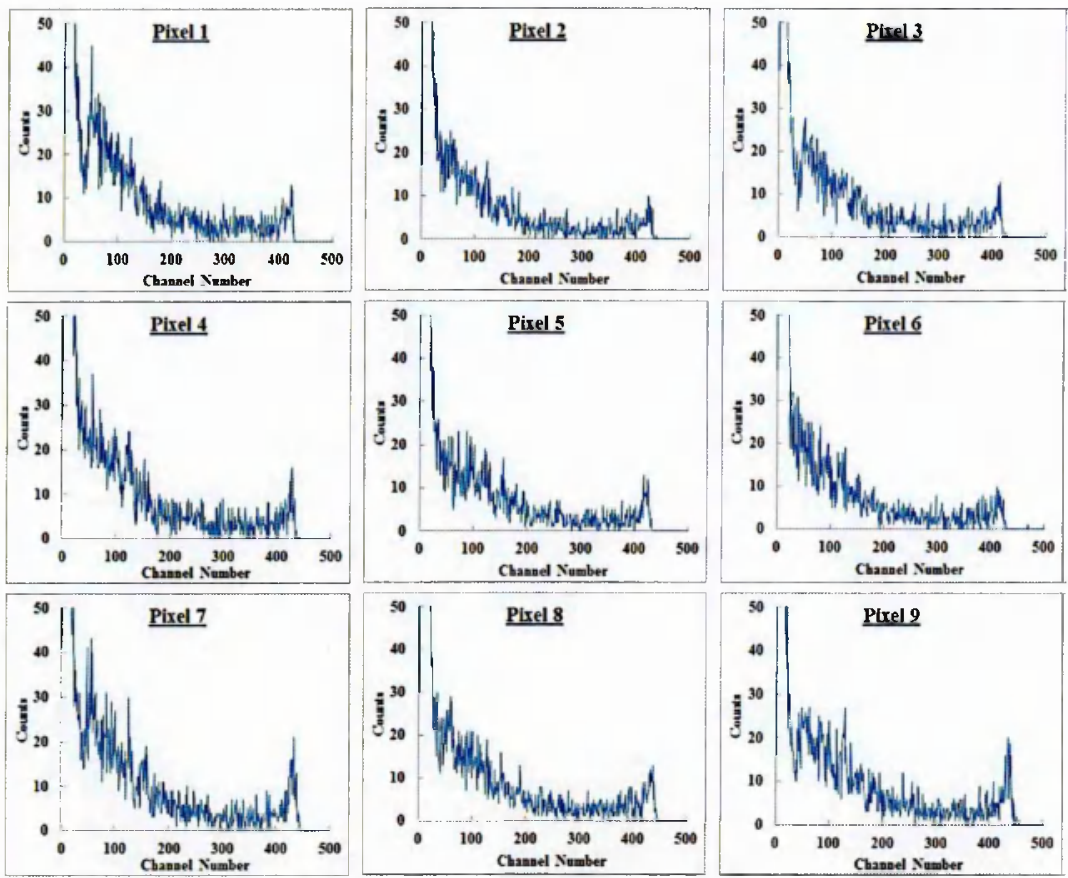

(a) Array 3
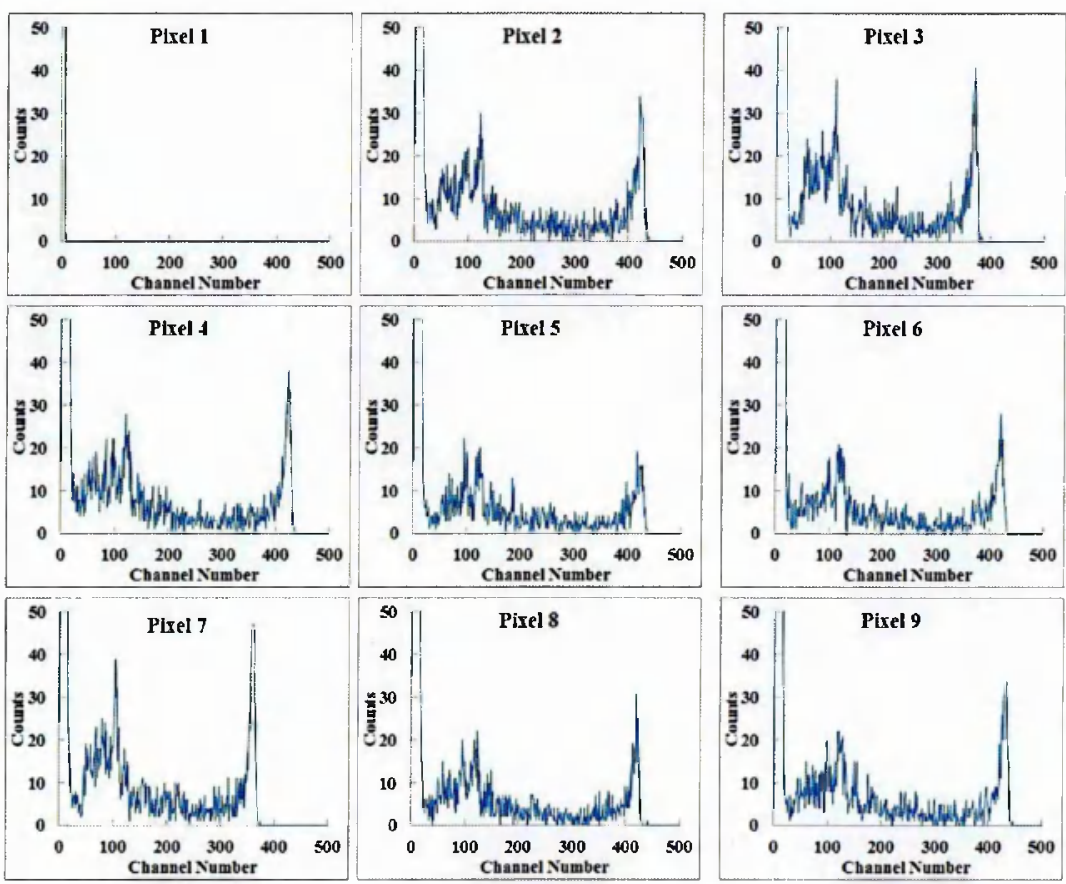

(b) Array 4

Figure 4.22: Uncalibrated single pixel spectra recorded by arrays 3 and 4. To show the effect different gains and offsets have on the resulting peak centroid channel number of the $59.5 \mathrm{keV}$ characteristic X-ray. 


\begin{tabular}{|c|c|c|}
\hline \multirow{2}{*}{ Pixel } & \multicolumn{2}{|c|}{ Peak Centroid(ch) } \\
\cline { 2 - 3 } & Array 3 & Array 4 \\
\hline 1 & 416.1 & N/A \\
\hline 2 & 422.1 & 420.7 \\
\hline 3 & 412.0 & 368.3 \\
\hline 4 & 426.8 & 421.6 \\
\hline 5 & 420.0 & 419.1 \\
\hline 6 & 415.8 & 418.4 \\
\hline 7 & 431.7 & 359.1 \\
\hline 8 & 429.7 & 413.6 \\
\hline 9 & 432.8 & 429.7 \\
\hline
\end{tabular}

Table 4.6: Peak centroid value for each pixel for the spectra shown in figure 4.22.

to a slight reduction of the gain for preamplifiers used for pixels 3 and 7 compared to other pixels.

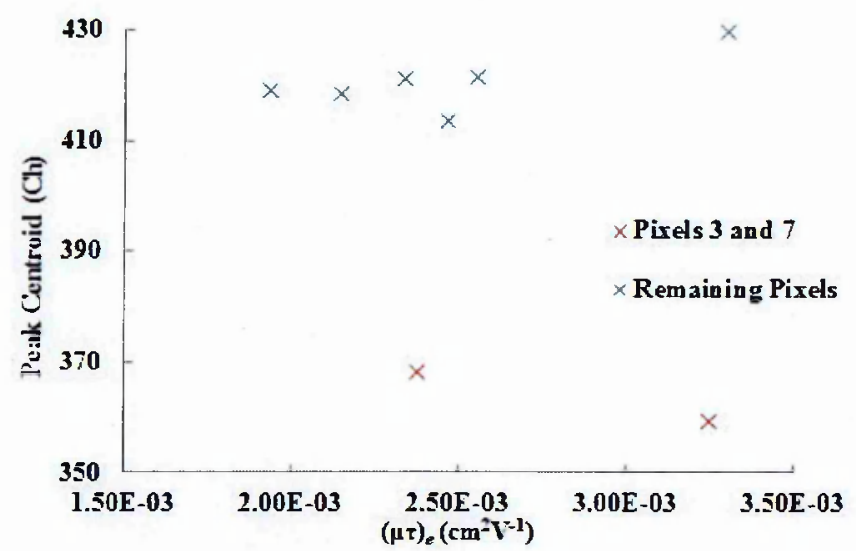

Figure 4.23: Peak centroid of the $59.5 \mathrm{keV}$ peak as function of the $(\mu \tau)_{e}$ for each pixel of array 4.

The difference in gains and offsets between pixels can effect the spectroscopic performance of a detector when the spectra generated by each pixel are summed together (see figure 4.24). As shown in figure 4.24 there are two different peaks relating to the detection of the $59.5 \mathrm{keV} \gamma$ ray. 'Peak A' is due to the detection of the $59.5 \mathrm{keV}$ $\gamma$ ray by pixels 3 and 7 . As shown in figure 4.25(a) the combined spectra collected by pixels 3 and 7 has a peak centroid channel number of 360.9. The remaining pixels spectra as shown in figure 4.25(b) has a peak centroid channel number of 421.2 results in 'Peak B' in figure 4.24. The effect the difference in gains and offsets has 


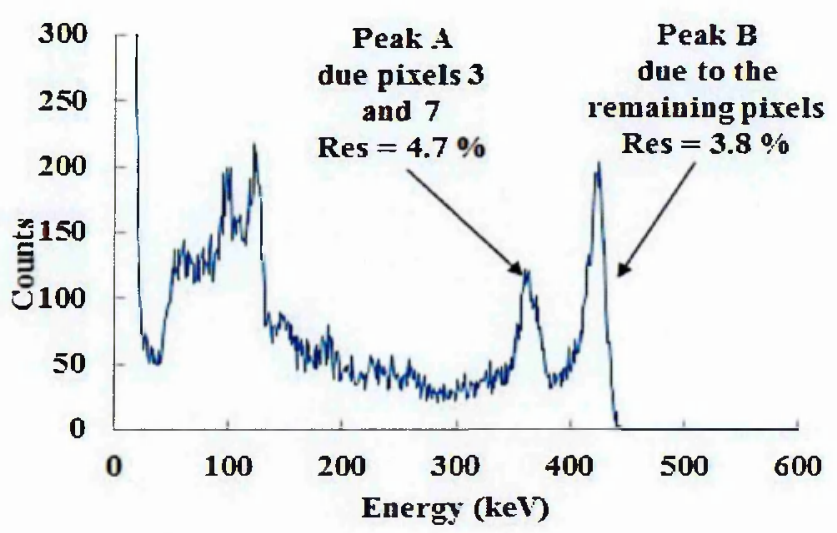

Figure 4.24: Spectrum produced by array 4 at a bias of $-300 \mathrm{~V}$ when the spectra recorded by each pixel are combined together. The two different $59.5 \mathrm{keV}$ peaks labelled 'Peak A' and 'Peak B' are due to differences in the gains of pixels 3 and 7 compared to the remaining pixels.

on the sum spectrum can be minimised by converting the generated channel number to a measured energy by applying the pixel gain and offset.

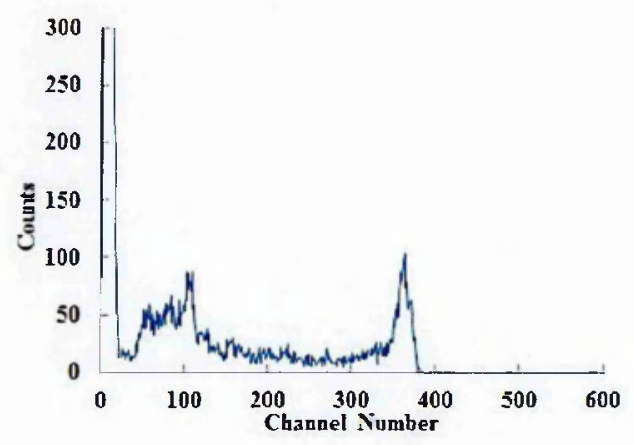

(a) Pixels 3 and 7

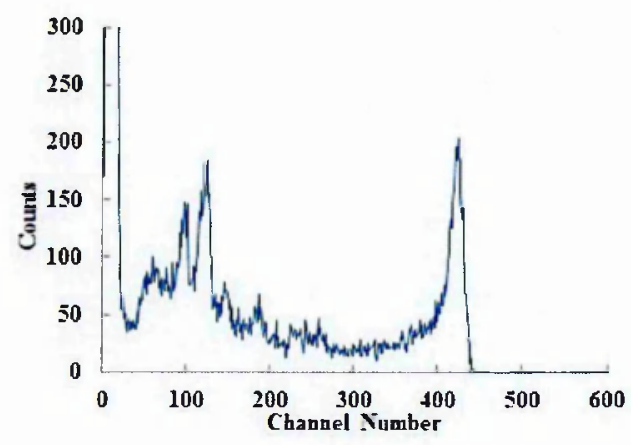

(b) Remaining pixels

Figure 4.25: Spectra recorded by pixels with different gains resulting in different peak centroid numbers and the non-gain matched sum spectrum shown in figure 4.24 .

The gain and offset of each pixel is calculated by creating a calibration curve for each pixel. A calibration curve makes it possible to convert a measured channel number to a measured energy. To generate this curve the peak centroids of the Silver $22.16 \mathrm{keV}$, Terbium $44.18 \mathrm{keV}$ characteristic $\mathrm{X}$ rays and ${ }^{241} \mathrm{Am} 59.5 \mathrm{keV} \gamma$ ray were recorded for each pixel and plotted against energy as shown in figure 4.26. The resulting gains and offset for each pixel are the gradient and the $y$-intercept 

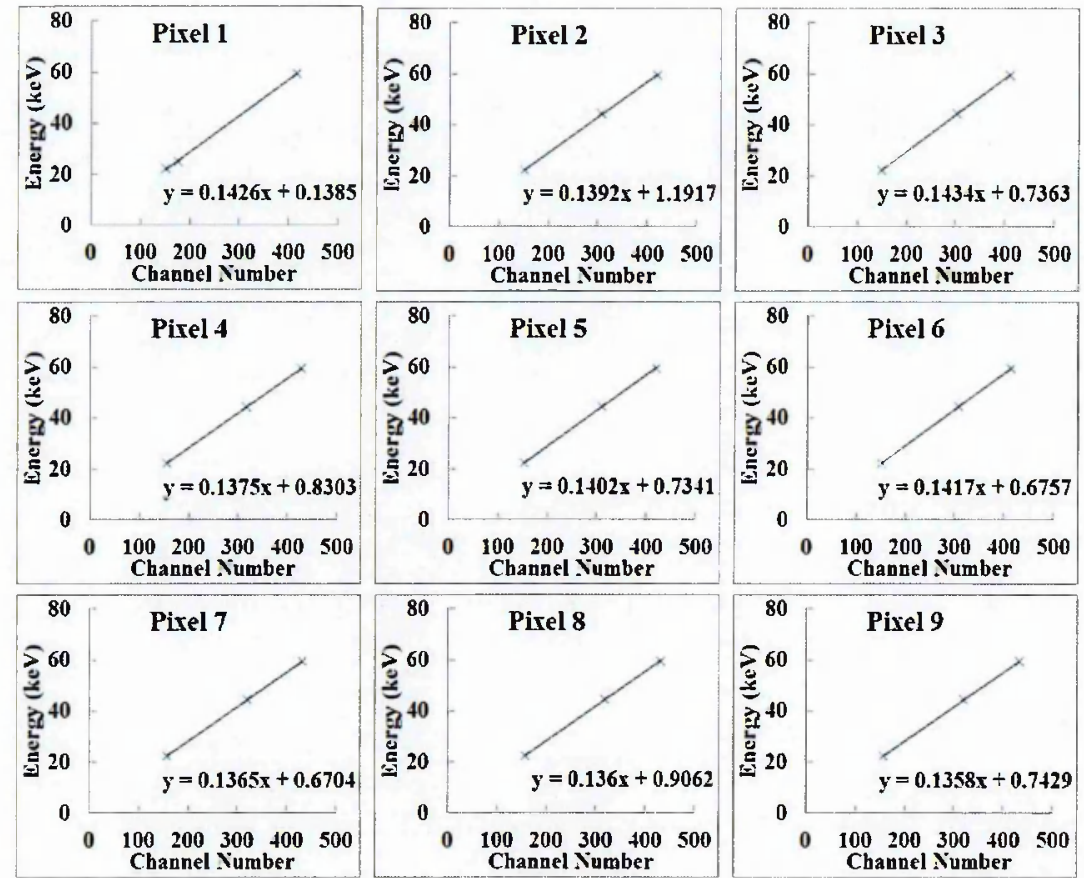

(a) Array 3
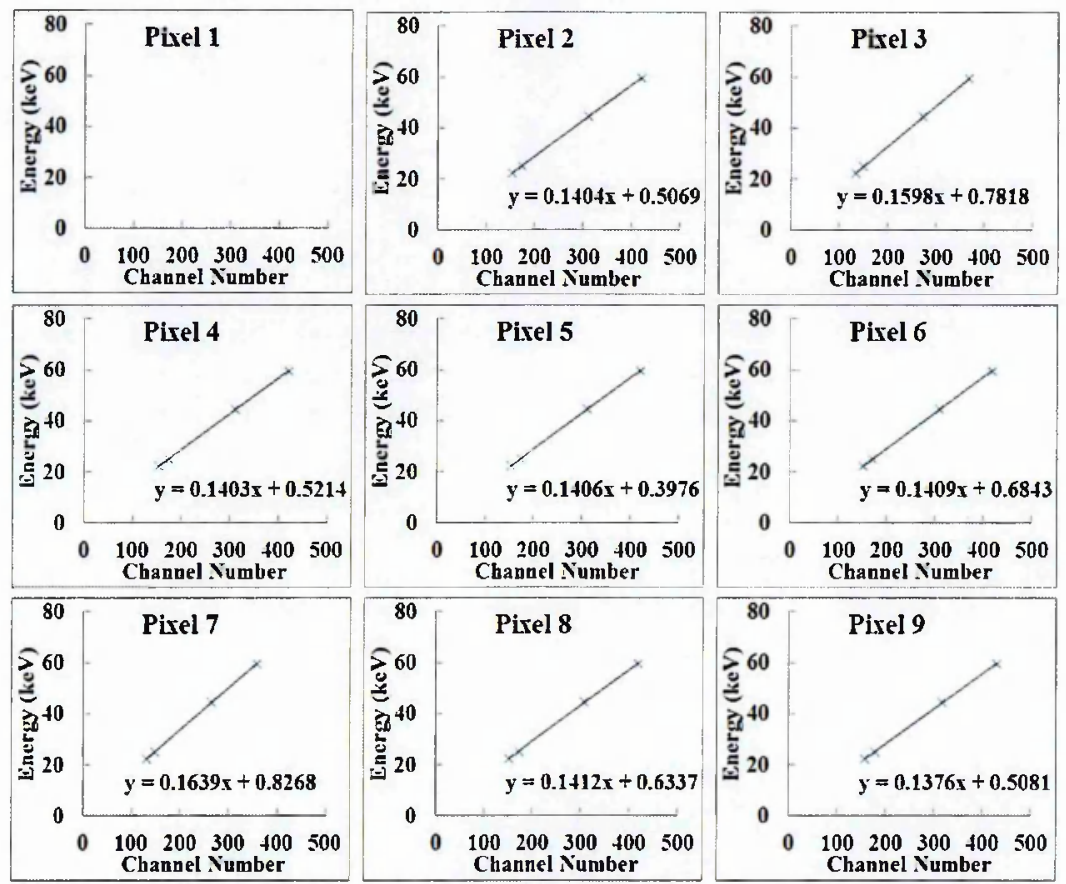

(b) Array 4

Figure 4.26: Pixel calibrations at $-300 \mathrm{~V}$ to calculate the gain matching parameters for arrays 3 and 4 of the PIXIE detector. 
of the linear regression respectively. Table 4.7 shows the resulting calculated gain matching parameters for arrays 3 and 4 at a detector bias of $-300 \mathrm{~V}$.

\begin{tabular}{|c|c|c|c|c|}
\hline \multirow{2}{*}{ Pixel } & \multicolumn{2}{|c|}{ Array 3} & \multicolumn{2}{c|}{ Array 4 } \\
\cline { 2 - 5 } & Gain $(\mathrm{keV} / \mathrm{ch})$ & Offset $(\mathrm{keV})$ & Gain $(\mathrm{keV} / \mathrm{ch})$ & Offset (keV) \\
\hline 1 & 0.143 & 0.14 & N/A & N/A \\
\hline 2 & 0.140 & 1.19 & 0.140 & 0.51 \\
\hline 3 & 0.144 & 0.74 & 0.160 & 0.78 \\
\hline 4 & 0.138 & 0.83 & 0.140 & 0.52 \\
\hline 5 & 0.140 & 0.73 & 0.141 & 0.40 \\
\hline 6 & 0.142 & 0.68 & 0.141 & 0.68 \\
\hline 7 & 0.137 & 0.67 & 0.164 & 0.83 \\
\hline 8 & 0.136 & 0.91 & 0.141 & 0.63 \\
\hline 9 & 0.136 & 0.74 & 0.138 & 0.51 \\
\hline
\end{tabular}

Table 4.7: Gain and offsets for gain matching for arrays 3 and 4

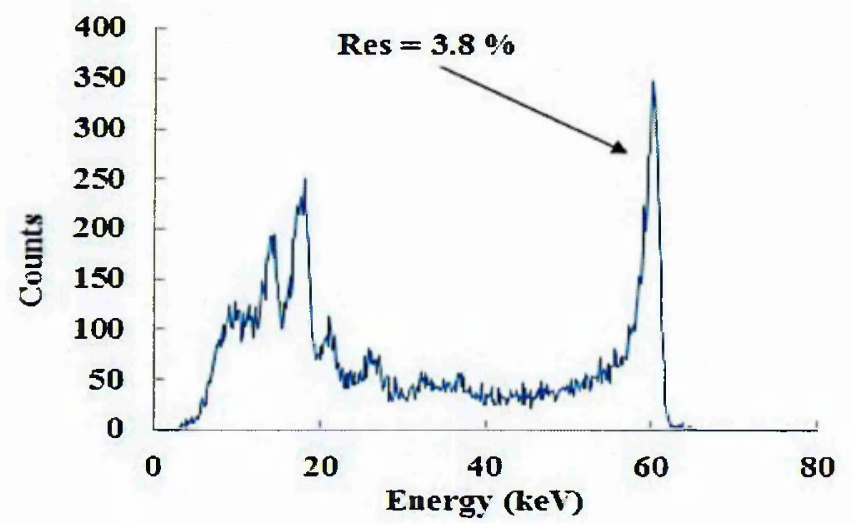

Figure 4.27: Spectrum produced by array 4 at a bias of $-300 \mathrm{~V}$ when the spectra recorded by each pixel are gained matched and summed together.

Once the gain and and offset on an event by event basis the channel number calculated by the $\mathrm{CR}-(\mathrm{RC})^{4}$ shaper is converted to a measured energy value using equation 4.7 where $G$ is gain and $O$ is the offset of the pixel. This results in the different channel number for the peak centroid of each pixel being adjusted to the same energy and resulting in the spectrum shown in figure 4.27 . The gain matching procedure greatly improves the spectroscopic performance resulting in a FWHM of $2 \mathrm{kcV}$ at $59.5 \mathrm{keV}$ and will result in more accurate values for the amount of charge sharing between adjacent pixels. 


$$
\mathrm{E}=(\text { Gain } \mathrm{x} \text { Channel Number })+\text { Offset }
$$

\subsubsection{Event Classification and the Optimum Threshold Value}

It is possible to classify a radiation event by the number of pixels which collects the charge. If the resulting CR-(RC) $)^{4}$ shaper amplitude for a pixel generates a channel number greater than a user defined threshold it is classified as a hit and it is assumed that charge has been collected by that pixel. The number of pixels with a valid hit in each event is known as the multiplicity $(m)$ of an event. When more than one pixel has a valid hit $(m>1)$ the event is classified as a charge sharing event.

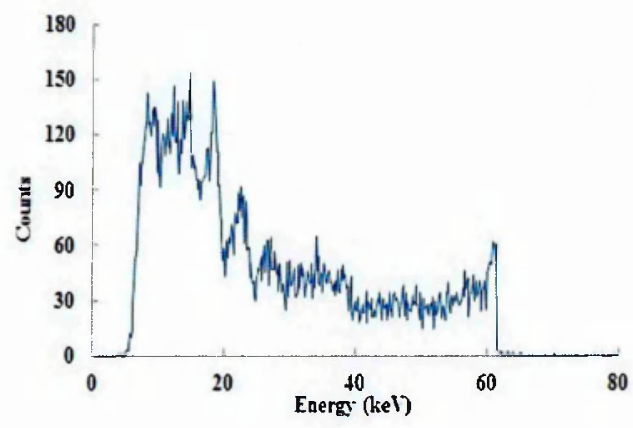

(a)

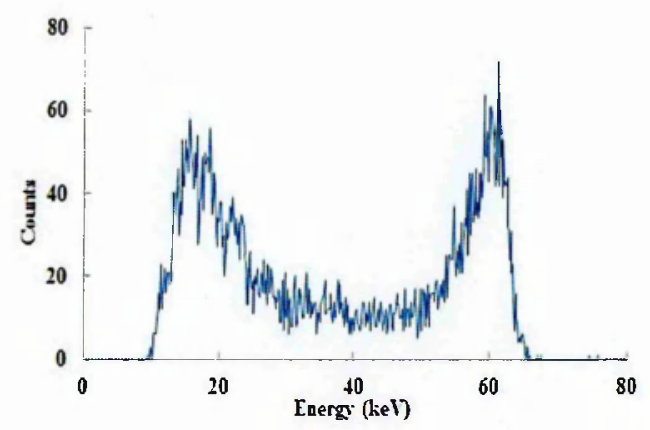

(b)

Figure 4.28: The same data processed in two different ways to show effect of sum spectrum generation. Spectrum (a) shows only the energy from the single pixel with the highest energy in a $m \geqslant 2$ event. Spectrum (b) shows the sum of the recorded energy from all pixels above the user defined threshold of $3 \mathrm{keV}$ in each $m \geqslant 2$ event

Once the multiplicity of an event is determined a sum spectrum can be created. On an event by event basis the total energy for each event is calculated by summing the energy of the pixels with valid hits. For example if the charge collected in charge sharing events was not summed together only the maximum energy collected by a single pixel would be plotted (see figure 4.28(a)). When the same data set is processed so charge sharing events are summed together (see figure $4.28(\mathrm{~b})$ ) the total collected charge is reconstructed resulting in a better spectroscopic performance. Before any detailed analysis can be carried out on charge sharing between pixels the optimum threshold value had to be found. $\mathrm{A}^{241} \mathrm{Am}$ spectrum was processed at 


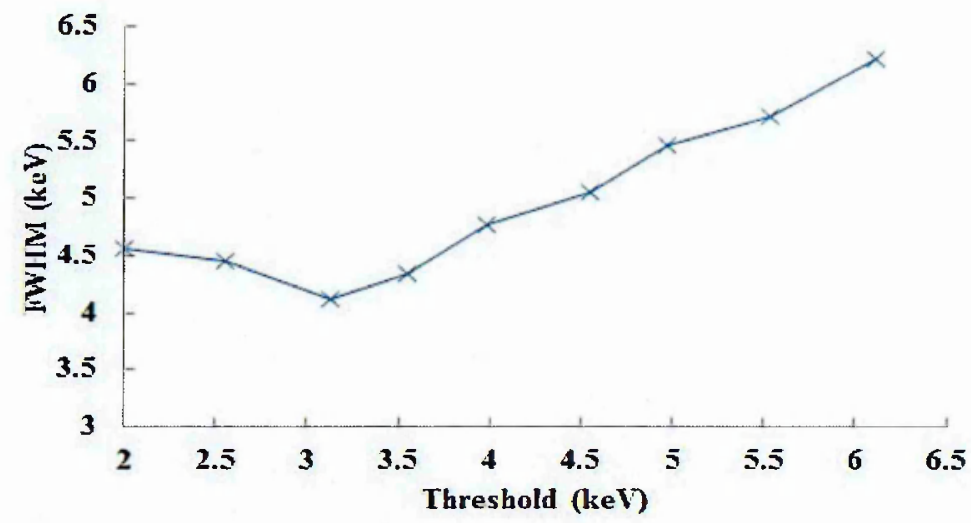

(a) All multiplicity $(m \geqslant 1)$ events

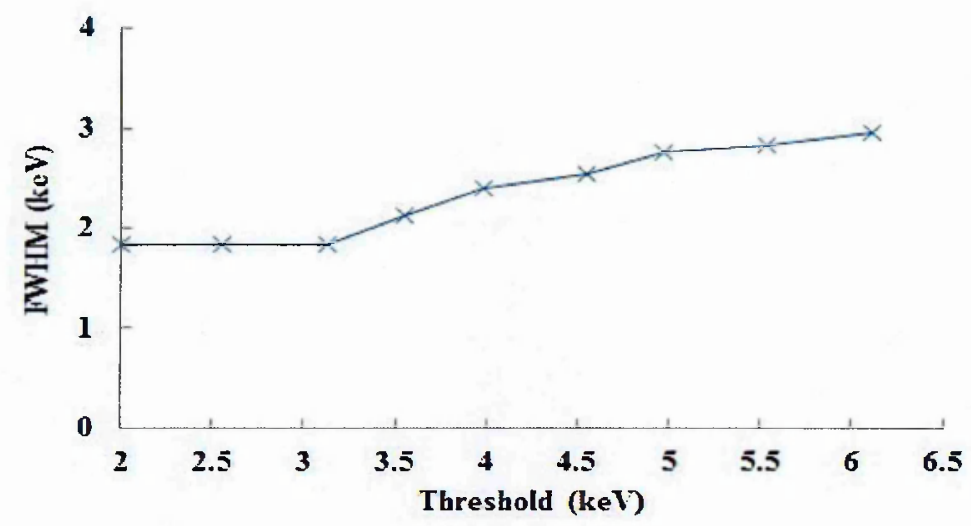

(b) $m=1$ events

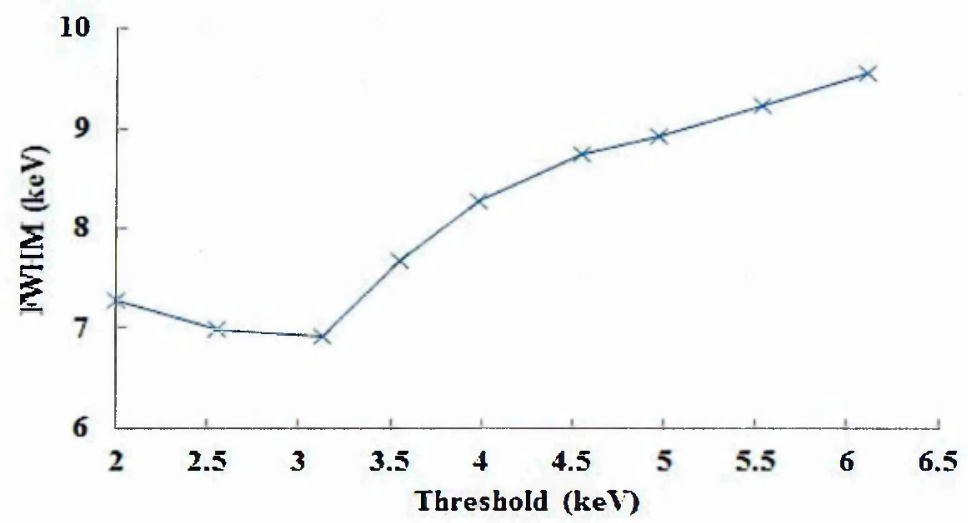

(c) $m=2$ events

Figure 4.29: FWHM of the $59.5 \mathrm{keV}$ peak from ${ }^{241}$ Am at different threshold values for the $m \geqslant 1, m=1$ and $m=2$ sum spectra. 
different threshold values and the FWHM of the characteristic $59.5 \mathrm{keV}$ peak was recorded for spectra involving all multiplicity events (figure 4.29(a)), $m=1$ events (figure 4.29(b)) and $m=2$ events (figure 4.29(c)).

As shown in figure 4.29(a) the FWHM for the sum spectrum with all multiplicities $(m \geqslant 1)$ is found to go through a minima at a value of $3 \mathrm{keV}$. At threshold values less than $3 \mathrm{keV}$ noise is classified as a hit and is falsely included into the sum spectrum. This also effects the $m=2$ threshold scan (figure $4.29(\mathrm{c})$ ) as actual $m=1$ events are being classified as $m=2$ events and being included in the $m=2$ spectrum.

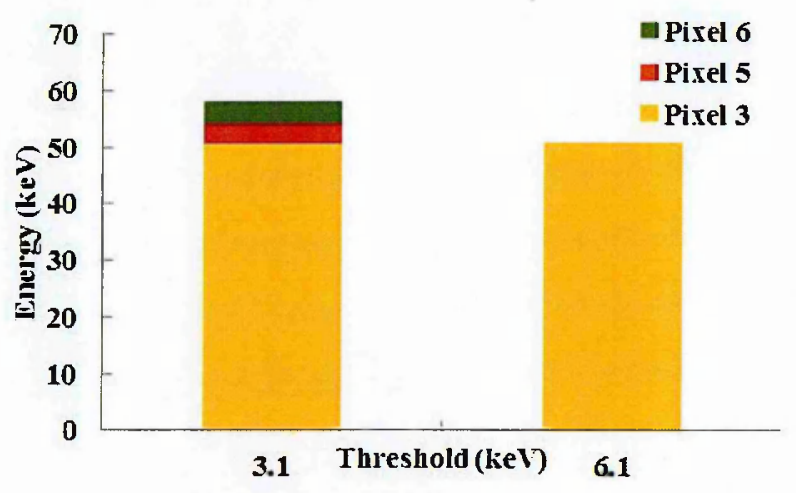

Figure 4.30: Components of a charge sharing event inputted to sum spectrum at different threshold values. Showing at higher threshold values real charge sharing events are neglected.

At threshold values greater than $3 \mathrm{keV}$ charge sharing events between neighbouring pixels are lost and the event is incorrectly classified with a lower multiplicity. For example figure 4.30 shows a $m=3$ charge sharing event which has 3 pixels with energies of $50.6 \mathrm{keV}, 3.5 \mathrm{keV}$ and $3.7 \mathrm{keV}$. At a threshold value of $6 \mathrm{keV}$ the two smaller pixel energies are lost from the total event energy, and the resulting spectrum will be degraded. The effect of neglecting small energy components of charge sharing is strongest in the $m=2$ spectrum and hence the $m \geqslant 2$ multiplicity spectra are most sensitive to correct adjustment of the threshold level.

\subsubsection{Charge Sharing and Sum Spectrum Performance}

A PIXIE detector has 4 different selectable arrays with different pixel sizes and pitches. This opens up the possibility to investigate how the pixel size effects the 
level of charge sharing and the observed detector performance. Data sets with the same number of events were taken with a ${ }^{241}$ Am source from arrays 3 and 4 which have the pixel sizes of $200 \mu \mathrm{m}$ and $350 \mu \mathrm{m}$ respectively and pitches of $250 \mu \mathrm{m}$ and $500 \mu \mathrm{m}$ respectively.

As shown in figure 4.31 there is a significant increase in the amount of charge sharing between pixels as the pixel pitch decreases. The reason for the increase in charge sharing at smaller pixel pitches is due to the width of the charge cloud. As explained in section 2.6.2 it is possible to model the diffusion of the charge carriers perpendicular to the propagation direction with a Gaussian distribution. This makes it possible to calculate charge cloud radius using equation 4.8. Where $r$ is the charge cloud radius, $k_{B}$ is Boltzmann's constant, $T$ is the absolute temperature, $D$ is the detector thickness, $d$ is charge cloud drifting distance, $e$ is the electronic charge and $V$ is the detector bias.

$$
r=1.15 \sqrt{\frac{2 k_{B} T d D}{e V}}
$$

The resulting charge cloud diameter for the interaction of $59.5 \mathrm{keV}$ with the detector at a bias of $-300 \mathrm{~V}$ will be $60 \mu \mathrm{m}$. Hence as this is much closer to the interpixel spacing for array 3 and significantly smaller than array 4 charge sharing is more likely to happen between pixels on array 3 .

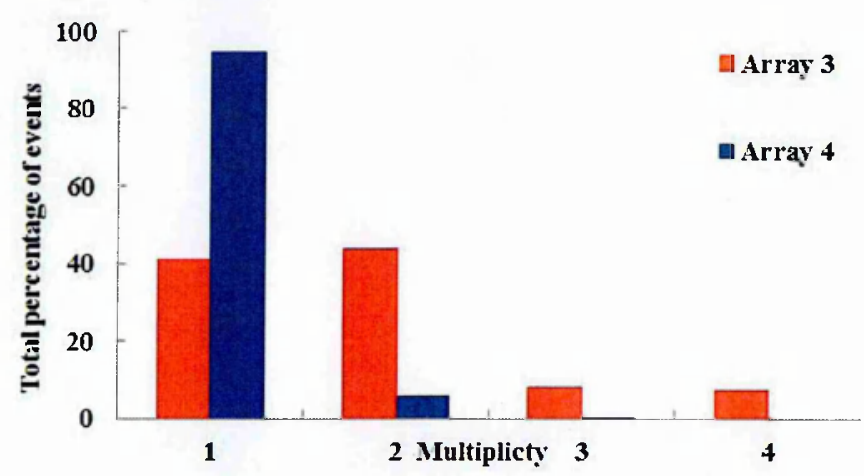

Figure 4.31: Multiplicity percentages recorded for array 3 and array 4 when irradiated with $59.5 \mathrm{keV}$ from ${ }^{241} \mathrm{Am}$ and a detector bias of $-300 \mathrm{~V}$.

The increased level of charge sharing between pixels also has a significant effect on the recorded spectroscopic performance of the detector. Figures 4.32 and 4.33 
show the recorded single and sum spectra recorded by arrays 3 and 4 with the subsequent FWHM for each spectrum shown in table 4.8. The increase in charge sharing results in a degradation of the spectroscopic performance of the single pixel and sum spectra.

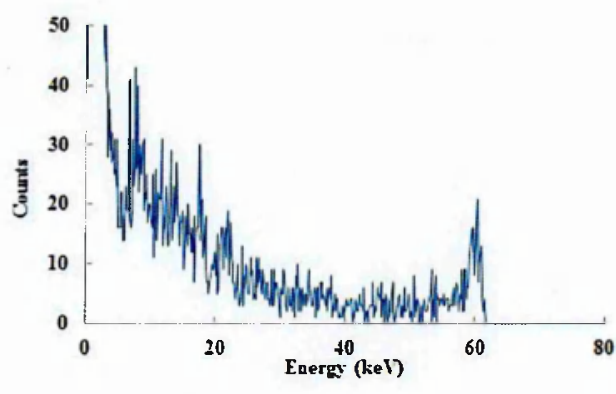

(a) Array 3

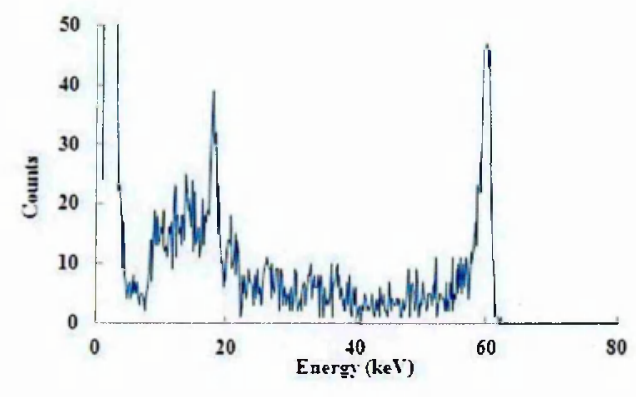

(b) Array 4

Figure 4.32: Single pixel spectra recorded by pixel 7 from arrays 3 and 4 to show effect increased charge sharing has on spectroscopic performance.

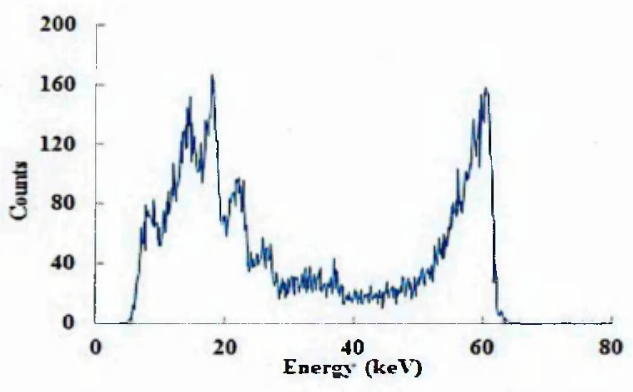

(a) Array 3

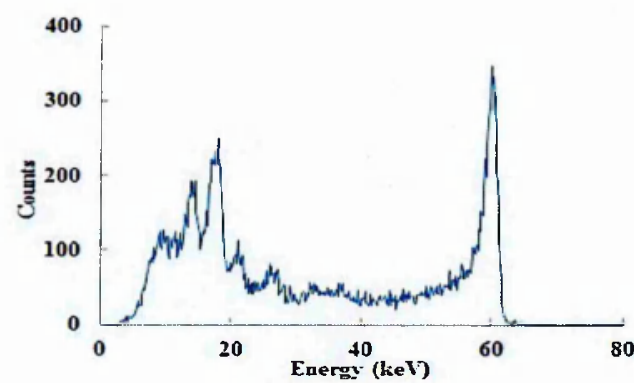

(b) Array 4

Figure 4.33: Sum spectra recorded by array 3 and 4 to show effect increased charge sharing has on spectroscopic performance.

To account for the degradation in the sum spectra performance it is possible to separate the spectra due to charge sharing events $(m \geqslant 2)$ from the spectrum generated by events with no charge sharing $(m=1)$. As shown in figure 4.34 the spectroscopic performance of the $m=1$ spectrum for array 3 (figure 4.34(a)) is significantly better than the $m \geqslant 2$ sum spectrum (figure 4.34(b)). However the increased spectroscopic performance that is achieved by neglecting $m \geqslant 2$ events results in a drop in efficiency which can be an undesirable property for some applications. 


\begin{tabular}{|c|c|c|}
\hline Array & $\begin{array}{c}\text { Single Spectrum } \\
\text { FWHM (keV) }\end{array}$ & $\begin{array}{c}\text { Sum Spectrum } \\
\text { FWHM (keV) }\end{array}$ \\
\hline 3 & 2.01 & 5.41 \\
\hline 4 & 1.56 & 2.00 \\
\hline
\end{tabular}

Table 4.8: FWHM of single and sum spectra shown in figures 4.32 and 4.33 respectively.

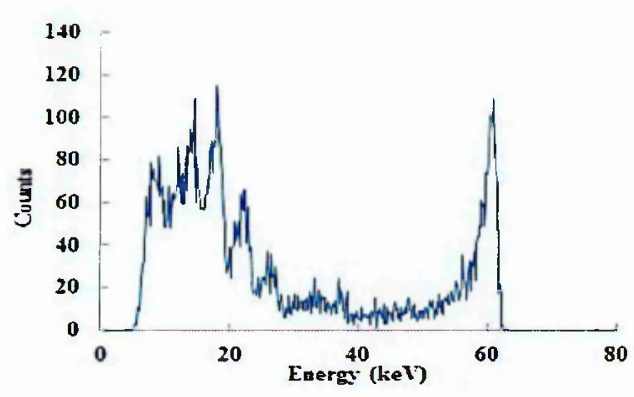

(a) $\mathrm{m}=1$ sum spectrum

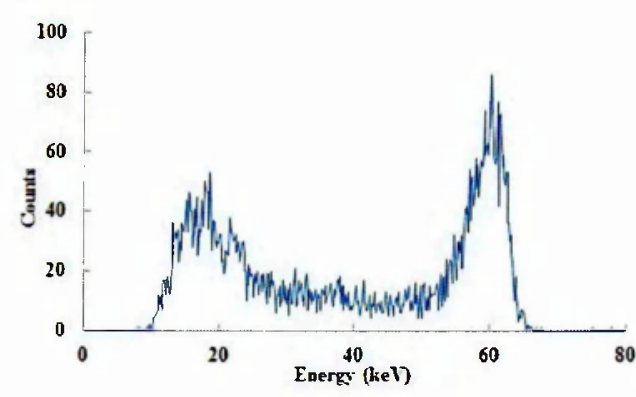

(b) $m \geqslant 2$ sum spectrum

Figure 4.34: The $m=1$ and $m \geqslant 2$ sum spectra rccorded by array 3 at $-300 \mathrm{~V}$ to show the effect of charge sharing on spectroscopic performance.

The amount of charge sharing between adjacent pixels was also measured for different incident X-ray energies. Array 3 of the PIXIE detector was irradiated with $22.16 \mathrm{keV}$ X-rays from Silver, $44.48 \mathrm{keV}$ X-rays from Terbium and $59.5 \mathrm{keV}$ X-rays from ${ }^{241} \mathrm{Am}$ and multiplicity of each event was recorded.

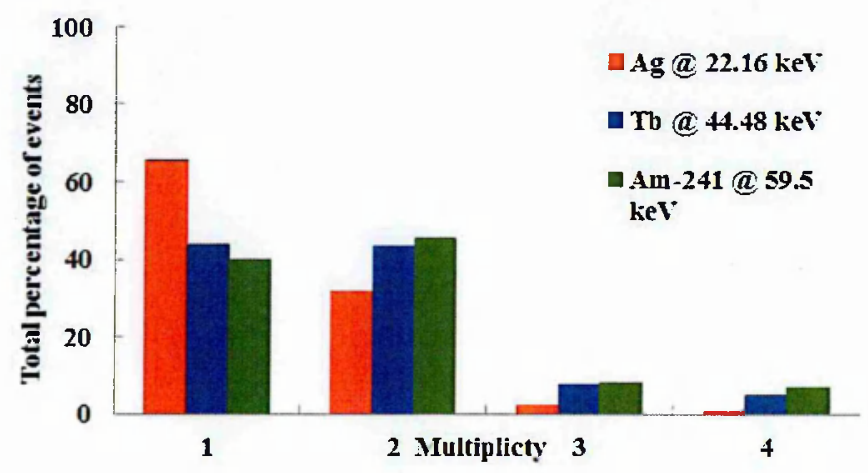

Figure 4.35: Multiplicity percentages recorded for varying incident X-ray energies for array 3 at $-300 \mathrm{~V}$.

Figure 4.35 shows the percentage of total events for each multiplicity at the different $\mathrm{X}$-ray energies. It was found that the amount of charge sharing increased as the energy increased. Furthermore there is a significant increase between $\mathrm{Ag}$ and $\mathrm{Tb}$. 
This is due to the creation of fluorescence X-rays as the incident X-ray energy is greater than the $K$-edge absorption energies for $\mathrm{Cd}(26.7 \mathrm{keV})$ and $\mathrm{Te}(31.8 \mathrm{keV})$. As explained in section 2.2 the resulting propagation of the fluorescence X-rays increases the size of the charge cloud. This effect will be investigated in section 5 and was the topic of the published paper in Appendix A [54].

\subsection{Summary}

This section details studies into the metrology and spectroscopy of pixelated CdZnTe radiation detectors. A metrology study was undertaken on a CdZnTe Medipix detector. A non-contact optical profiler (Zygo) was used to measure the curvature of the device. It was found the curvature diagonally across the sample was of the order to $16-18 \mu \mathrm{m}$. This amount of curvature is significant as the diameter of the In bumps fabricated to the Medipix III ASIC will be of the order of $16-20 \mu \mathrm{m}$. This may result in corner pixels not being electrically contacted to the ASIC resulting in dead areas.

The surface roughness of the bare CdZnTe sample was also measured. The average roughness value $R_{a}$ of the sample was calculated by performing a line scan in the interpixel region of two pixels. An $R_{a}$ value of $6.56 \mathrm{~nm}$ was recorded for the sample. As a result of this the surface roughness of the sample will have no effect on the In bump bonding procedure.

The last metrology study undertaken was to investigate the gold pixels fabricated to the CdZnTe material. As shown in figure 4.7 it was found that there were multiple pixels which exhibited increased height of gold pixels on the right hand and bottom edges. It was found for the pixel shown in figure 4.8 that the spike measured due to the increased gold was $154 \mathrm{~nm}$ higher that the $260 \mathrm{~nm}$ gold pixel. To investigate whether this was a real effect or artefact of the Zygo the pixels were investigated in a Scanning Electron Microscope. As shown in figure 4.9 there is evidence of increased gold height on the right and bottom edges. The origin of this effect is thought to be due an issue in the lift-off procedure in the fabrication of the gold pixels on the 
CdZnTe material.

A spectroscopy study was also undertaken on a Redlen CdZnTe PIXIE detector. To begin with the ${ }^{241} \mathrm{Am}$ spectra generated by a single pixel (see figure 4.12) was used to optimise the parameters used in the digital CR-(RC) ${ }^{n}$ shaper. It was found the optimum value for the time constant $(\tau)$ of the shaper was $0.5 \mu$ s (see figure 4.13). The is due to series and parallel electronic noise having more effect on the resulting spectra at values of $\tau$ less than and greater than $0.5 \mu$ s respectively. The number of stages of integration $(n)$ also needed to be optimised. It was found that the spectroscopic performance of the single pixel spectrum improved up to a value of $n=4$ (see figure 4.16) and subsequently degraded at higher values of $n$. This is due to at a value $n=4$ the signal-to-noise ratio of the shaper output was at a minium.

Once the parameters of the digital shaper had been optimised the effect of the detector bias on the spectroscopic performance of a single pixel from array 4 was undertaken. It was found that the FWHM of the $59.5 \mathrm{keV}$ peak improved with detector bias (see figure 4.18). Due to the low level of charge sharing between adjacent pixels for this array the two major components on the resulting FWHM is the electronic noise and the charge collection efficiency (CCE). It was found that the leakage current increases with detector bias which would have a negative effect on the FWHM, however the CCE was found to improve with detector bias which resulted in the improved spectroscopic performance at higher detector biases. This as result shows that the CCE has a larger effect on the spectroscopic performance of the detector.

The analysis software also has the ability to investigate multiple pixel effects. To begin with a gain matching procedure had to be carried out. The discrepancy in gains between pixels is due to slight differences in the gains of PIXIE ASIC preamplifier's and differences in the charge transport properties as shown in table 4.5. The difference in gain and offsets result in a significant decrease in spectroscopic performance (see figure 4.24). This can be accounted for by converting the generated channel number into an energy resulting in an improved spectroscopic performance. 
The analysis software also has the ability to classify an event by the number pixels with an equivalent energy greater than a user defined threshold which is known as the multiplicity. The sum of the equivalent energies can be plotted in a sum spectrum. The optimum threshold value was found to be $3 \mathrm{keV}$. At values less than $3 \mathrm{keV}$ noise was classified as a hit and inputted into the sum spectrum, at values greater than $3 \mathrm{keV}$ small components of charge sharing were not put into the sum spectrum thus degrading the spectroscopic performance.

Finally charge sharing between adjacent pixels was investigated. It was found that for smaller pixel pitches the level of charge sharing was found to increase (see figure 4.31). This is due to the charge cloud created by $59.5 \mathrm{keV} \mathrm{X}$-ray at a detector bias of $-300 \mathrm{~V}$ has a final size comparable to the interpixel gap of array 3 thus making charge sharing more likely. Furthermore the increased level of charge sharing significantly decreased the spectroscopic performance of the detector. The charge sharing between the pixels of the same array was found to increase with incident $\mathrm{X}$-ray energy. There was a significant increase for X-rays energies greater than the $K$-edge absorption energies for $\mathrm{Cd}(26.7 \mathrm{keV})$ and $\mathrm{Te}(31.8 \mathrm{keV})$. This is due to the subsequent emission and propagation of fluorescence $\mathrm{X}$-rays which increases the initial size of the charge cloud. This effect will be investigated in depth in the next section of this thesis. 


\section{Chapter 5}

\section{Focused X-ray Beam Studies}

There are a large number of interesting effects observed when using pixelated radiation detectors as a way of characterising radiation. Effects such as charge sharing between adjacent pixels and charge loss in the interpixel region can have a negative effect on the performance of a radiation detector. Pixelated detectors can also be used to define the interaction position of the radiation with the detector medium. To investigate all these effects line scans between adjacent pixels were carried out on the I15 beamline at the Diamond Light Source, UK.

One investigation which will explained in this chapter is how the level of charge sharing between adjacent pixels is effected by emission of fluorescence X-rays. The propagation length of the fluorescence X-rays is large $(\sim 100 \mu \mathrm{m})$ and subsequently increases the initial charge cloud size. To investigate this effect on the level of charge sharing between pixels line scans between adjacent pixels were carried out with $\mathrm{X}$ ray beam energies above (40 keV) and below (26 keV) the mean $K$-shell absorption energy of the detector materials $(26.7 \mathrm{keV}, 9.7 \mathrm{keV}$ and $31.8 \mathrm{keV}$ for $\mathrm{Cd}, \mathrm{Zn}$ and $\mathrm{Te}$, respectively).

Another interesting effect investigated in this section is charge loss in the interpixel region. On each position of the line scan the total collected energy is recorded and how this values varies with interaction position will be investigated. The use of a pixelated detector to locate where an X-ray interacted in a detector will also be discussed. The spectrum collected by each pixel will be used to define where an 
interaction took place by a interpolation scheme developed in this project.

Finally the effect the detector bias has on the charge sharing between adjacent pixels will also be investigated in this chapter.

\subsection{Array and Pixel Locating}

Before any line scans between adjacent pixels could be undertaken the arrays and pixels of interest had to be located on the CdZnTe sample. During the fabrication of the PIXIE arrays onto the CdZnTe sample the position of the arrays on the sample in relation to the diecast aluminium box was recorded. This meant it was possible to locate the area at which array 4 of the PIXIE detector was thought to be.

To find the centre of array 4 a $26 \mathrm{keV}$ X-ray beam with a mean spot size of $2 \mathrm{~mm}$ was moved in $50 \mu \mathrm{m}$ steps in the $X$ (horizontal) and $Z$ (vertical) directions. At each position on the scan the output from the centre pixel (pixel 5) was analysed with the software built into the Pixie-4 DAQ system explained in section 3.4. The software has the ability to calculate the number of counts/second a pulse is processed with an amplitude above a user defined threshold. This value makes it possible to locate the centre of an array as the number of counts/second will increase as the X-ray beam gets closer to the centre pixel of the array (pixel 5). To support the data recorded by the Pixie- 4 system the pulse generated by the pixel 5 of the array of interest on the PIXIE ASIC was observed on an oscilloscope.

Figure 5.1(a) shows the recorded counts/second for pixel 5 at each point on the scan to find the centre of the array. At some points on the scan (the red zone in figure 5.1) the Pixie-4 software outputted no value, furthermore the pulses observed on the oscilloscope at these positions showed evidence of pile up when the rate at which events are being detected is too fast. At these positions it was assumed the beam was over the centre of the array. As the centre of array 4 had been found the box was moved $2 \mathrm{~mm}$ in the negative $X$ direction and the same procedure was carried out on array 3. In this case the centre of the array was found to be at a value of $X$ $=-0.5 \mathrm{~mm}$ and $Z=2.75 \mathrm{~mm}$. 


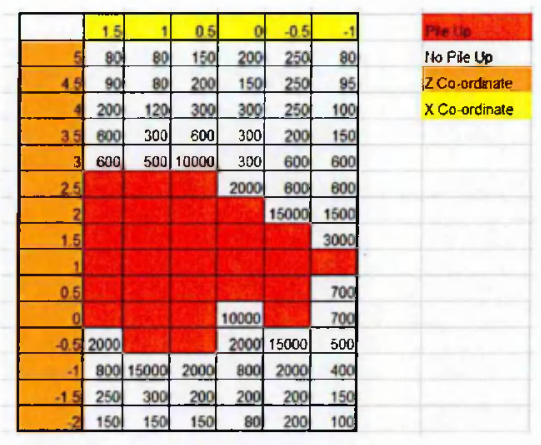

(a) Array 4

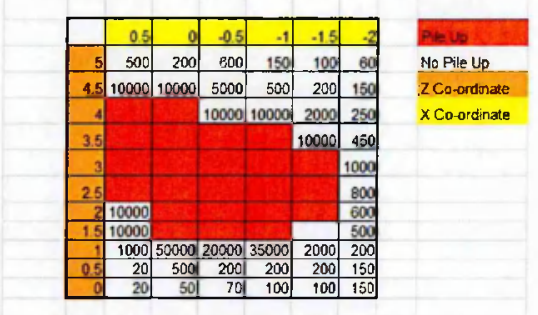

(b) Array 3

Figure 5.1: Counts/second recorded by pixel 5 on each point of scan to find centre of array 3 and 4 of the Redlen CdZnTe PIXIE detector. Colour code relates to the resulting pulse seen on the oscilloscope.

\begin{tabular}{|c|c|c|c|c|}
\hline Pixels & $\begin{array}{c}\text { Results } \\
\text { Figure }\end{array}$ & $\begin{array}{c}X \text { Co- } \\
\text { ordinate }(\mathrm{mm})\end{array}$ & $\begin{array}{c}\bar{Z} \text { Co- } \\
\text { ordinate }(\mathrm{mm})\end{array}$ & $\begin{array}{c}\text { Centre of Pixel 5 } \\
\text { Co-ordinate }(\mathrm{mm})\end{array}$ \\
\hline 1,2 and 3 & $5.3(\mathrm{a})$ & -0.5 & $2.45 \rightarrow 3.4$ & $\mathrm{~N} / \mathrm{A}$ \\
\hline 2,5 and 8 & $5.3(\mathrm{~b})$ & $-0.55 \rightarrow-0.95$ & 2.9 & $\mathrm{X}=-0.75$ \\
\hline 4,5 and 6 & $5.3(\mathrm{c})$ & -0.75 & $2.675 \rightarrow 3.075$ & $\mathrm{Z}=2.875$ \\
\hline
\end{tabular}

Table 5.1: Details of the line scans to find the centre of pixel 5 of array 3 .

Once the centre of array 3 had been located three line scans were carried out with the $26 \mathrm{keV}$ X-ray beam collimated to a mean spot size of $20 \mu \mathrm{m}$ (see table 5.1 for information on line scans). The purpose of these scans was to locate the centre of pixel 5 of array 3 of the Redlen PIXIE detector.

At each position on the three line scan a list mode file was created by the Pixie-4 DAQ. This list mode file contained information on the pulses generated by all nine pixels when an X-ray interacted with the detector. As explained in section 3.5 a piece of LabVIEW software was developed to analyse the pulses recorded in the list mode file. One ability of the LabVIEW software is to record how many pixels collected charge created from the interaction of an X-ray with the detector. If the total collected charge by a pixel is above a user defined threshold (in this case 3 $\mathrm{keV}$ ) the pixel is classified as a hit. The number of pixels classified as a hit in a specific event is known as the multiplicity $(m)$ of the event. For example if the event is classified as an $m=1$ event all the charge generated by the interaction of an $\mathrm{X}$ ray with the detector material is collected by a single pixel. 


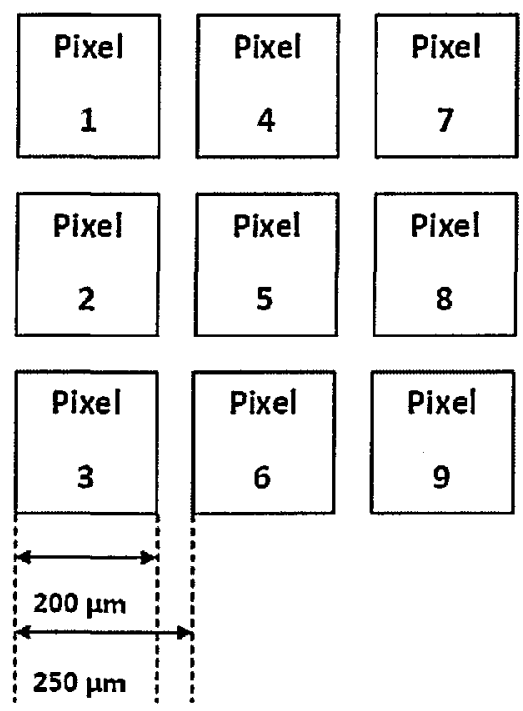

Figure 5.2: Orientation of the pixel numbers for array 3 of PIXIE detector as seen by the I15 beamline.

The first line scan carried was between pixels 1,2 and 3 (see figure 5.2 for pixel orientation and size). As shown in figure 5.3(a) the beam was moved in $50 \mu \mathrm{m}$ steps and at each position the total number of $m=1$ events was recorded, and number of times each pixel was involved in one was recorded. As shown there are regions where $100 \%$ of $m=1$ events are found to be attributed to only one pixel. The centre of these regions is assumed to be when the beam was incident on the centre of that pixel. Therefore the centre of pixel 2 was found to be at an $Z$-co-ordinate of $2.9 \mathrm{~mm}$.

The second line scan was then carried out to find the centre of pixel 5 in the $X$ direction. The box was fixed at a $Z$ co-ordinate of $2.9 \mathrm{~mm}$ and was moved between pixels 2,5 and 8 in $25 \mu$ steps. As shown in figure 5.3(b) the centre of the region where $100 \%$ of $m=1$ events was attributed to pixel 5 was found at a co-ordinate of $X=-0.75 \mathrm{~mm}$. This is then assumed to be the centre of pixel 5 in this direction. The final line scan was to find the centre in the $Z$ direction. The detector was fixed at an $X$ co-ordinate of $-0.75 \mathrm{~mm}$ and was moved in the $Z$ direction in $25 \mu \mathrm{m}$ steps between pixels 4,5 and 6 . The centre of the region where $100 \%$ of $m=1$ events was attributed to pixel 5 was found at a position of $Z=2.875 \mathrm{~mm}$. 


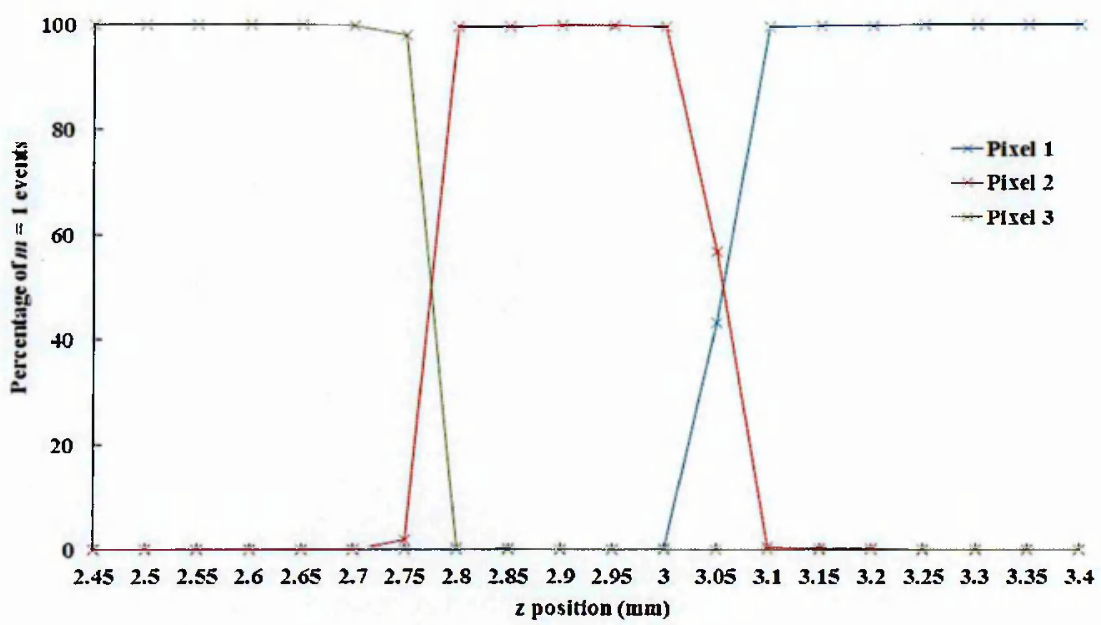

(a) Line scan between pixels 1, 2 and 3 .

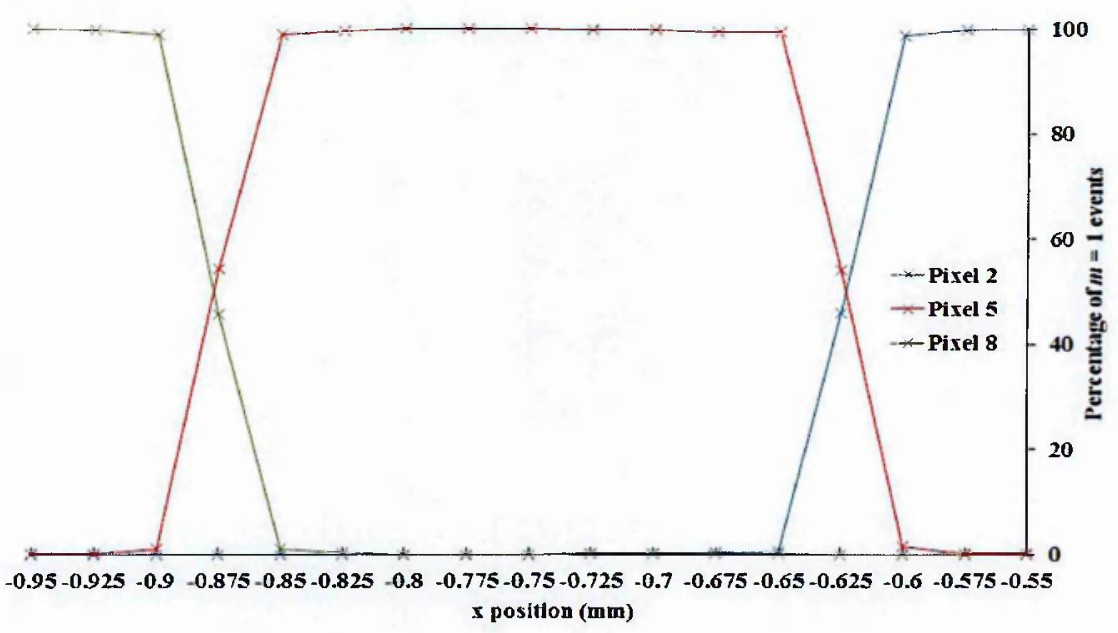

(b) Line scan between pixels 2,5 and 8 .

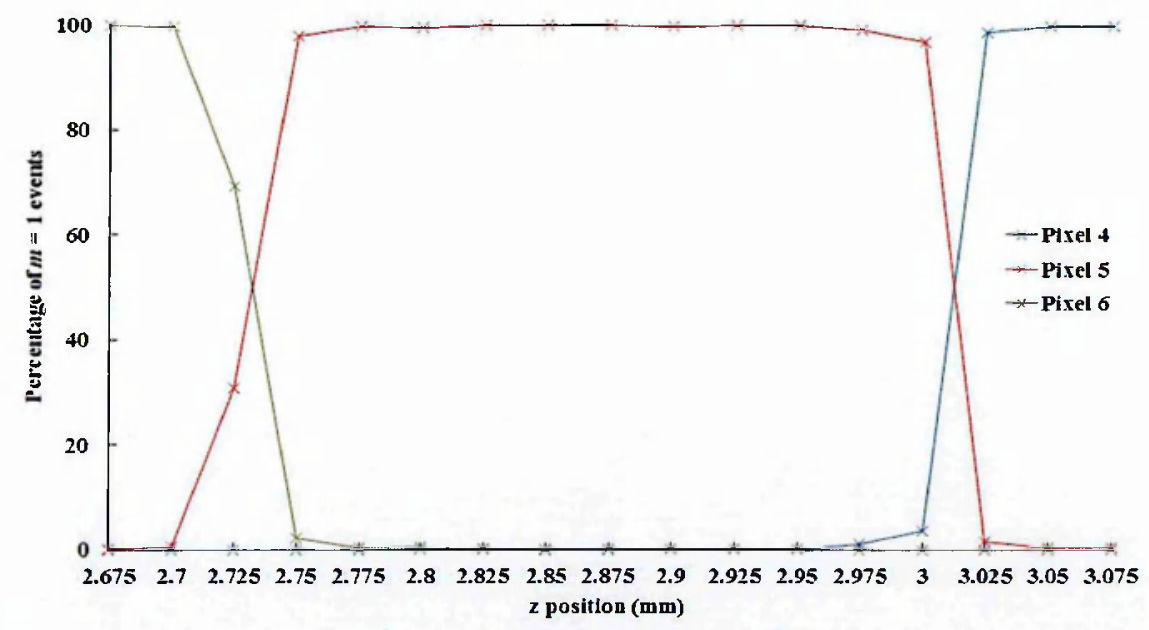

(c) Line scan between pixels 4,5 and 6 .

Figure 5.3: The three line scans carried out to find the centre of pixel 5 of array 3 of the Redlen PIXIE detector. 
Therefore the centre of pixel 5 was found to be located at a co-ordinate of $X=-0.75$ $\mathrm{mm}$ and $Z=2.875 \mathrm{~mm}$ and line scans between the centres of pixels 4 and 5 of array 3 could be undertaken.

\subsection{Single Pixel Spectra}

On each position on the line scan between pixels 4 and 5 the spectrum produced by each pixel was recorded. Figure 5.4(a) shows the spectrum produced by pixel 5 when the $26 \mathrm{keV}$ was incident on the centre of the pixel. There is a full energy photopeak at $26 \mathrm{keV}$ with a FWHM of $1.1 \mathrm{keV}$ which is within $0.1 \mathrm{keV}$ of the FWHM due to the PIXIE ASIC as measured in section 4.2.1.1. For comparison the spectrum produced by pixel 5 when a $40 \mathrm{keV} \mathrm{X-ray} \mathrm{beam} \mathrm{was} \mathrm{incident} \mathrm{on} \mathrm{the} \mathrm{centre} \mathrm{of} \mathrm{the}$ pixel is shown in figure 5.4(b). The FWHM of the photopeak is $1.15 \mathrm{keV}$ however there are three additional peaks produced labelled " $\mathrm{B}$ ", " $\mathrm{C}$ " and " $\mathrm{D}$ ". These peaks are to due the creation and subsequent escape of $\mathrm{Cd}$ and $\mathrm{Te}, K_{\alpha}$ and $K_{\beta} \mathrm{X}$ rays. Table 5.2 shows the energy of the specific fluorescence $\mathrm{X}$ rays and the peak label in figure 5.4(b).Another difference between the single pixel spectra generated at 26 $\mathrm{keV}$ and $40 \mathrm{keV}$ is the peak-to-valley ration of the full energy peak. As shown in table 5.3 the peak-to-valley ratio for the $40 \mathrm{keV}$ peak is 5 times smaller than the one calculated for the $26 \mathrm{keV}$ spectrum. The exact mechanism being undertaken with this effect is not fully known and should be investigated at a future date.

The single pixel spectra recorded at beam energies of $26 \mathrm{keV}$ and $40 \mathrm{keV}$ both have peaks at around $2 \mathrm{keV}$ labelled "A" in both graphs This is due to the detection of transient pulse. An example of transient pulse is shown in figure 5.5. In this example a $76 \mathrm{keV}$ photon interacts with the detector resulting the pulse generated by the main pixel however there was a small additional pulse generated on a neighbouring pixel. This transient pulse is due to the charge cloud moving through the weighting potential of a neighbouring pixel and inducing a small bipolar pulse on this pixel. As previously explained in section 2.6.2 the net charge induced by a transient pulse is zero by the time the main pulse reaches its peak. This is due to the peaking time 


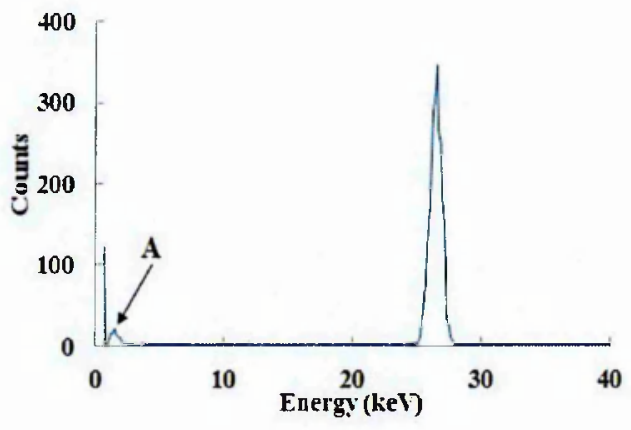

(a) $26 \mathrm{keV}$

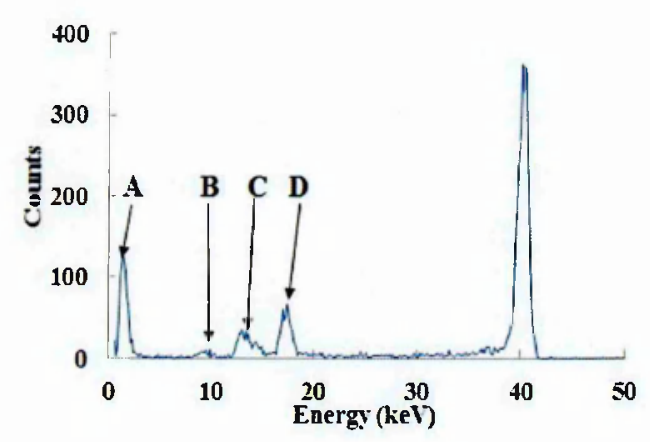

(b) $40 \mathrm{keV}$

Figure 5.4: Single pixel spectrum recorded by pixel 5 when the $\mathrm{X}$ ray beam was incident on the centre of the pixel.

\begin{tabular}{|c|c|c|c|c|}
\hline Element & Fluorescence X-ray & $\begin{array}{c}\text { Fluorescence X-ray } \\
\text { Energy (keV) }\end{array}$ & $\begin{array}{c}\text { Escape Peak } \\
\text { Energy (keV) }\end{array}$ & $\begin{array}{c}\text { Peak in } \\
\text { figure 5.4(b) }\end{array}$ \\
\hline \multirow{2}{*}{$\mathrm{Te}$} & $K_{\beta}$ & 31.00 & 9.00 & $\mathrm{~B}$ \\
\cline { 2 - 5 } $\mathrm{Cd}$ & $K_{\alpha}$ & 27.47 & 12.57 & $\mathrm{C}$ \\
\cline { 2 - 5 } & $K_{\beta}$ & 26.10 & 13.90 & $\mathrm{D}$ \\
\hline
\end{tabular}

Table 5.2: Fluorescence X-ray energies and subsequent escape peaks shown in figure 5.4 (b) for $\mathrm{Cd}$ and Te.

of the transient pulse is earlier the the main pulse as shown in the inset of figure 5.5. Therefore the full deposited charge in an event is represented by the peak amplitude of the main pulse and is not effected by the creation of transient pulses.

However the resulting amplitude of the transient pulse is a few $\mathrm{keV}$ which incorrectly produces the unexpected peak "A" at around $2 \mathrm{keV}$ in figure 5.4. Therefore to correct for this peak the LabVIEW software was adapted to ignore any transient pulse. Furthermore any noise pulses were ignored by applying a noise threshold of $1 \mathrm{keV}$ (sec figure figure 5.6). This as result made it possible to calculate the total charge deposited for event whilst ignoring any transient or noise pulses.

Figure 5.5 shows the pulse generated by a $76 \mathrm{keV}$ photon interacting with the $\mathrm{CdZnTe}$ detector. In the example pulse, a small additional pulse was generated on a neighbouring pixel. This transient pulse is due to the charge cloud moving through the weighting potential of a neighbouring pixel and inducing a small bipolar pulse on this pixel. As previously explained in section 2.6 .2 the net charge induced by a 


\begin{tabular}{|c|c|c|c|}
\hline $\begin{array}{c}\text { X-ray Energy } \\
(\mathrm{keV})\end{array}$ & \multirow{2}{*}{$\begin{array}{c}\text { FWHM } \\
(\mathrm{keV})\end{array}$} & \multicolumn{2}{|c|}{ Peak-to-Valley Ratio } \\
\cline { 3 - 4 } & 1.10 & 45 & $\begin{array}{l}\text { Value } \\
\text { Peak } 24 \mathrm{keV}-27 \mathrm{keV} \\
\text { Valley } 14 \mathrm{keV}-24 \mathrm{keV}\end{array}$ \\
\hline 26 & 1.15 & 9 & $\begin{array}{c}\text { Peak } 37 \mathrm{keV}-42 \mathrm{keV} \\
\text { Valley } 27 \mathrm{keV}-37 \mathrm{keV}\end{array}$ \\
\hline 40 &
\end{tabular}

Table 5.3: FWHM and Peak-to-Valley ratio of single pixel spectra recorded when the $26 \mathrm{keV}$ or $40 \mathrm{keV} \mathrm{X}$-ray beam was incident on the centre of pixel 5 .

transient pulse is zero by the time the main pulse reaches its peak. This is due to the peaking time of the transient pulse is earlier the the main pulse as shown in the inset of figure 5.5. Therefore the full deposited charge in an event is represented by the peak amplitude of the main pulse and is not effected by the creation of transient pulses.

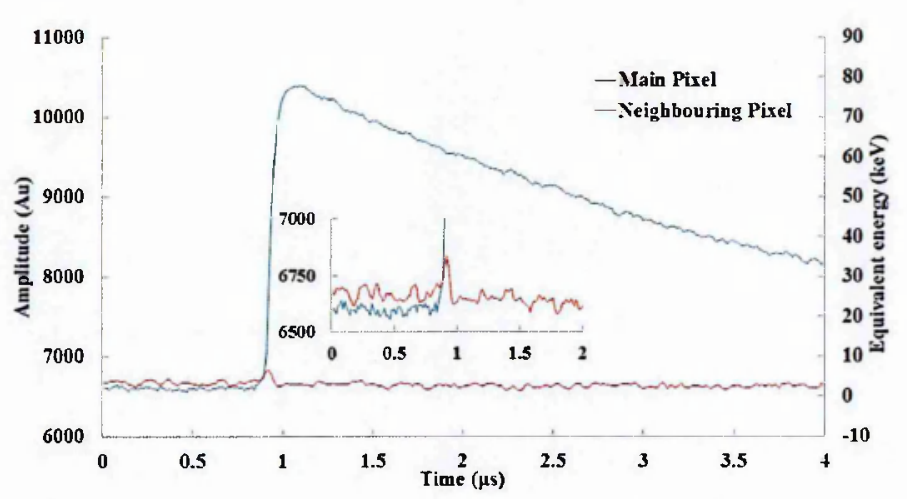

Figure 5.5: Typical pulse shape and associated transient pulse induced on a neighbouring pixel.

However the resulting amplitude of the transient pulse is a few $\mathrm{keV}$ which incorrectly produces the unexpected peak "A" at around $1.5 \mathrm{keV}$ in figure 5.4. Therefore to correct for this peak the LabVIEW software was adapted to ignore any transient pulse. Furthermore any noise pulses were ignored by applying a noise threshold of $1 \mathrm{keV}$ (see figure figure 5.6). This as result made it possible to calculate the total charge deposited for event whilst ignoring any transient or noise pulses. 


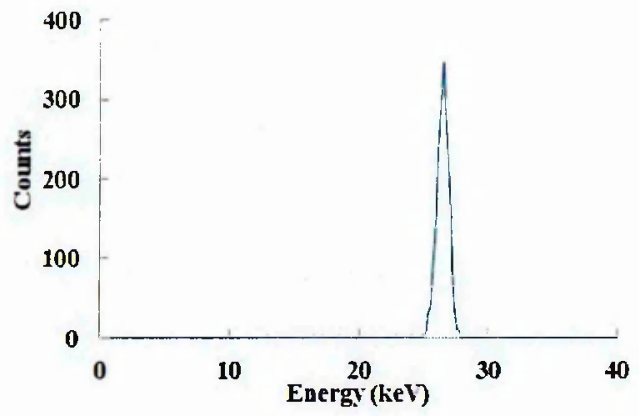

(a) $26 \mathrm{keV}$

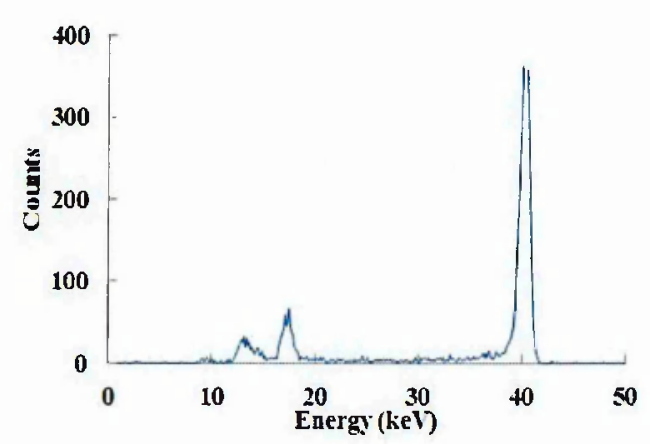

(b) $40 \mathrm{keV}$

Figure 5.6: Single pixel spectrum recorded by pixel 5 for the X-ray beam incident on the centre the pixel with transient pulses removed and noise threshold of $1 \mathrm{keV}$.

\subsection{Variation in Charge Collected by a Single Pixel}

Charge sharing between adjacent pixels can have a significant effect on the measured spectroscopic performance of a pixelated radiation detector. For example figure 5.7 (a) shows the single pixel spectra recorded by pixels 4 and 5 when a $26 \mathrm{keV}$ beam was incident on the edge of pixel 5. As shown both pixel spectra have peaks at cnergies significantly below the $26 \mathrm{keV} \mathrm{X}$-ray beam line energy which is due to the splitting of the charge generated in each event between the two pixels. To account for the high level of charge sharing and the subsequent degradation in detector performance a sum spectrum is created. As explained in scction 4.2 .2 on an event by event basis the energies of pixels which are above the software defined threshold are added together to form a total summed energy. This recovers the majority of the original photon energy. For example figure 5.7(b) shows the pixel spectra produced by pixels 4 and 5 when a $26 \mathrm{keV}$ beam was incident on the edge of pixel 5 . A $1 \mathrm{keV}$ software threshold is applied and a full energy peak is calculated at a value of 24.2 $\mathrm{keV}$. The full energy photopeak is less than $26 \mathrm{keV}$ which is due to charge being lost in the interpixel region. This effect will be investigated in the next section.

To investigate how the total collected charge varies with beamline interaction position line scans were performed between the centre of pixel 5 and centre of pixel 4 at a detector bias of $-300 \mathrm{~V}$ and with X-ray energies of $26 \mathrm{keV}$ and $40 \mathrm{keV}$. At each position the spectra collected by pixels 4 and 5 and the subsequent sum spectrum 


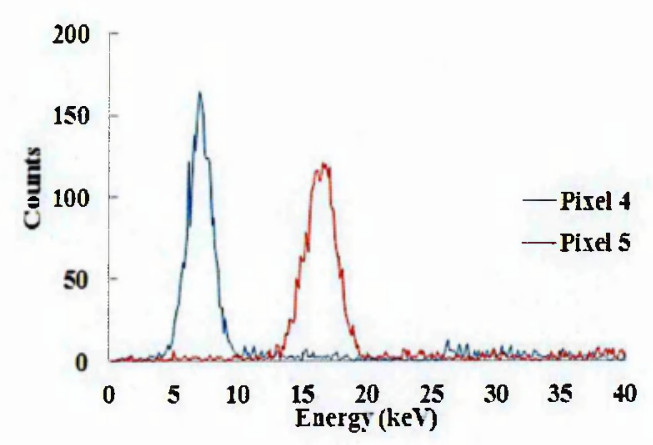

(a)

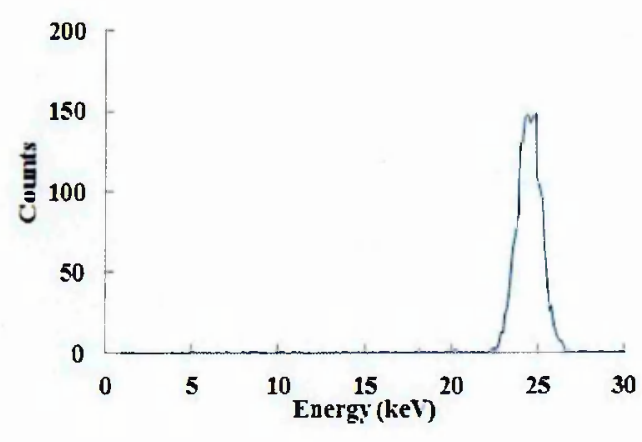

(b)

Figure 5.7: (a) Separate spectra recorded by pixels 4 and 5, showing full energy peaks at $7.4 \mathrm{keV}$ and $16.8 \mathrm{keV}$ respectively. (b) sum spectrum from the same data showing the full peak at an energy of $24.2 \mathrm{keV}$

was recorded.

Figure 5.8 shows how the peak centroid of pixels 4 and 5 vary with interaction position. It is possible to define the interaction position of the X-ray beam by the use of equation 5.1 , where $P_{4}(x), P_{5}(x)$ are the peak centroids of the pixels 4 and 5 respectively at a distance $x$ away from the centre of pixel 5 . As shown in figure 5.9 when this ratio is plotted as a function of interaction position the resulting linear relation can be used to define the position of the X-ray beam in the interpixel region. For example the centre of the interpixel region can be defined where the charge deposited is equally shared between pixels 4 and 5 . In this case $P_{4}(x)=P_{5}(x)$ resulting in a $R(x)$ value of 0 . For the case of the $26 \mathrm{keV}$ and $40 \mathrm{keV}$ line scans the centre of the interpixel region is found to be a distance of $126 \mu \mathrm{m}$ from the centre of pixel 5. This shows high accuracy in the model as the actual centre is $125 \mu \mathrm{m}$ from the centre of pixel 5 .

$$
R(x)=\frac{\left[P_{5}(x)-P_{4}(x)\right]}{\left[P_{5}(x)+P_{4}(x)\right]}
$$




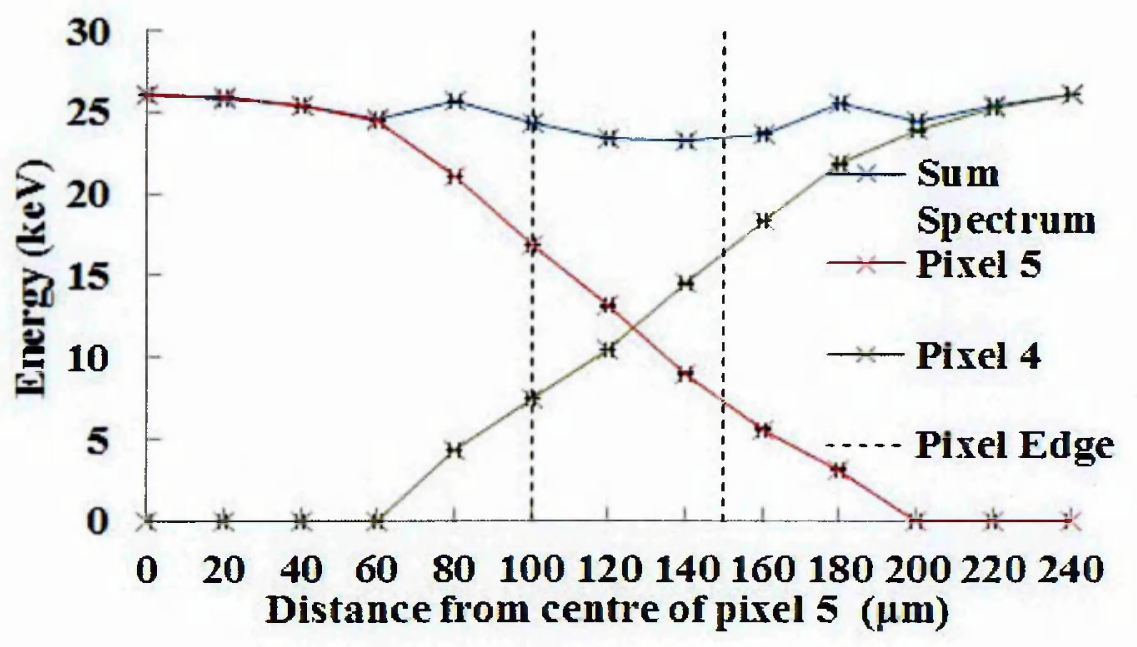

(a) $26 \mathrm{keV}$

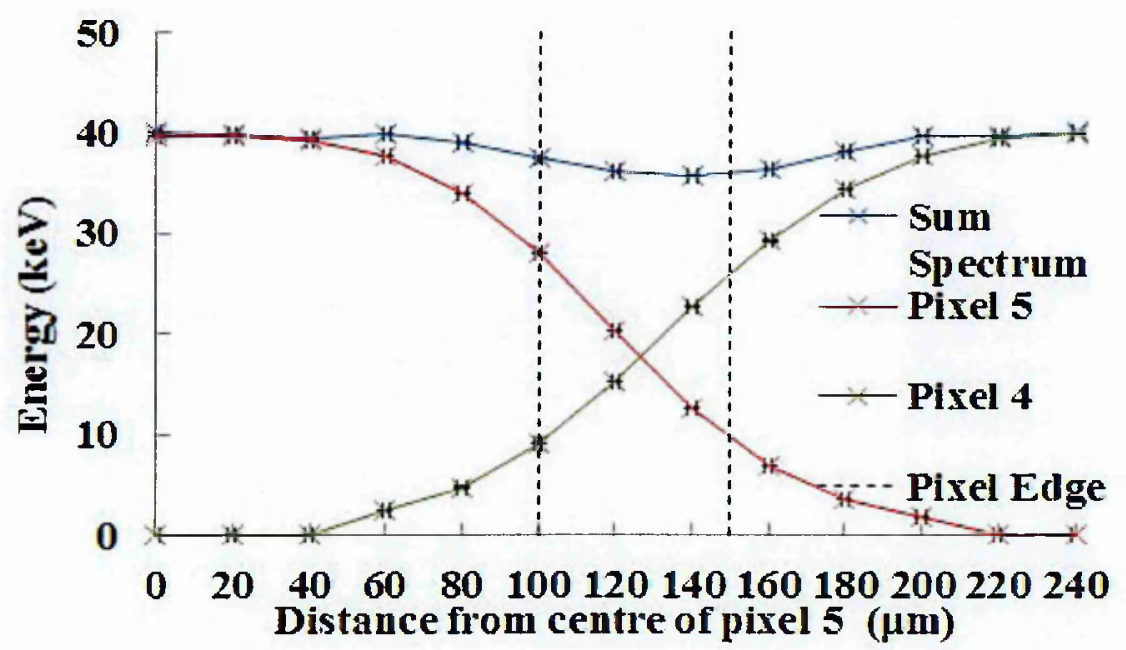

(b) $40 \mathrm{keV}$

Figure 5.8: The peak centroid energy of pixel 5, pixel 4 and the resulting sum spectrum at a detector bias of $-300 \mathrm{~V}$ and X-ray energies below $(26 \mathrm{keV})$ and above (40 keV) the $K$-shell absorption energies of $\mathrm{Cd}(26.7 \mathrm{keV})$ and Te $(31.8 \mathrm{keV})$. 


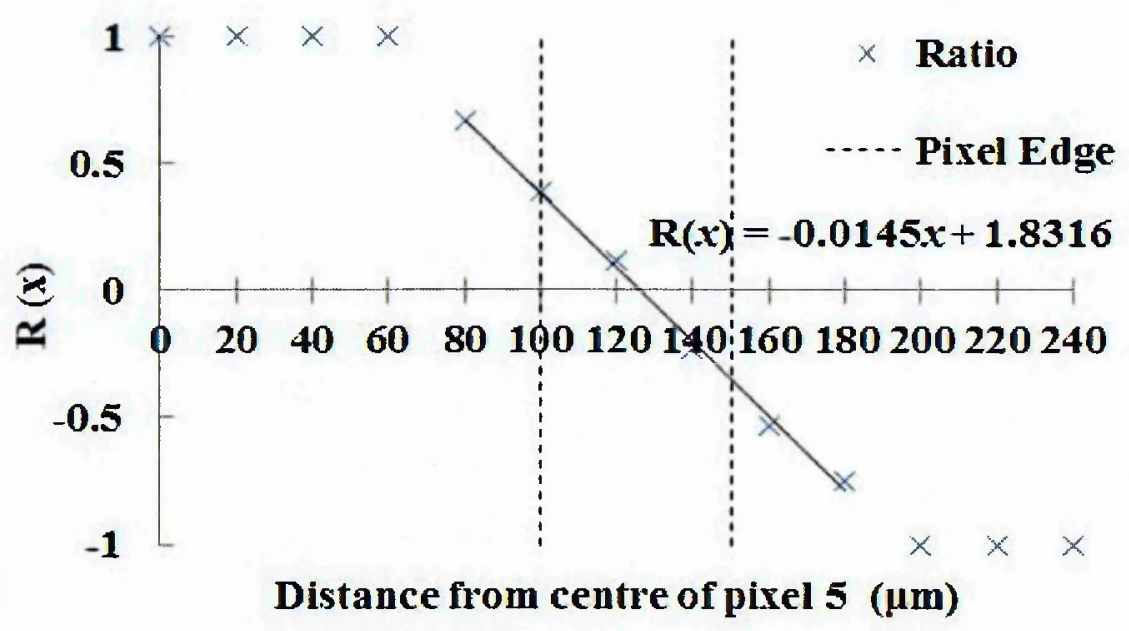

(a) $26 \mathrm{keV}$

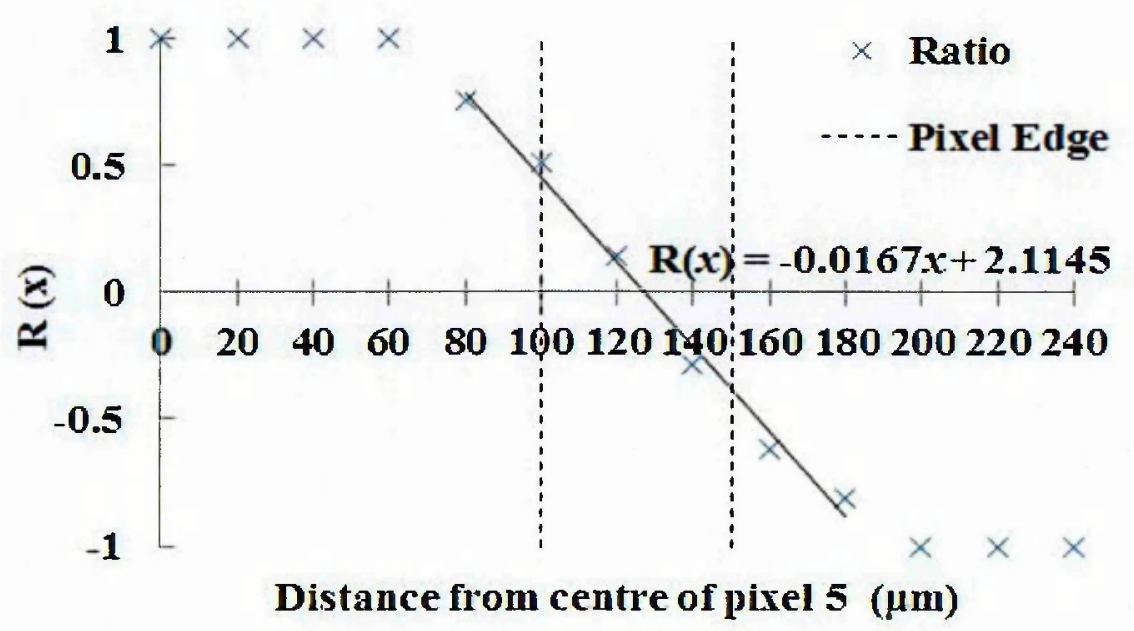

(b) $40 \mathrm{keV}$

Figure 5.9: Linear relationship between the charge sharing ratio $\mathrm{R}(x)$ and the position of the X-ray beam in the interpixel region. 


\subsection{Charge Loss in the Interpixel Region}

Figure 5.8 shows how the peak centroid of the sum spectrum changes with interaction position. The peak centroid of the sum spectrum is a measure of the amount of the total collected charge. In the interpixel region there is a observed drop in the amount of total collected charge. The charge loss is defined as the difference between the beamline energy and the peak centroid of the sum spectrum in keV. Figure 5.10 shows the measured charge loss at each position on the line scan between pixels 4 and 5 at a beamline energy of $26 \mathrm{keV}$ (figure 5.10(a)) and $40 \mathrm{keV}$ (figure 5.10(b)). The charge loss is due to electrons being trapped in a region close to the CdZnTe surface. Figure 5.11 is a TCAD simulation [53] of the electric field profile across the width of the device at beamline positions on the middle of a pixel and in the middle of the interpixel region [52]. As shown, the electric field strength is the same across the majority of the device. However in a region near the anode electrodes the electric field profiles change dependant on where the X-ray beam is incident. For X-ray beam positions incident on the centre of a pixel the electric field increases by $\sim 10 \%$ (figure 5.11(a)), in comparison for positions in the interpixel region the electric field strength drops by almost $100 \%$ (figure 5.11(b)). The drop in electric field in the interpixel region results in electrons being trapped and the subsequent charge loss is observed. Several authors have attributed the drop in electric field is due to the presence of a lower conductivity surface layer in the interpixel region $[55,56]$.

The threshold value can also result in a second different way for charge not to be included to the calculated total collected charge. At points "A" on figure 5.10(a) and points " $\mathrm{B}$ " on figure 5.10(b) the measured charge loss spikes. This is due to a small component of charge sharing which is below the software threshold of $1 \mathrm{keV}$ and is therefore not recorded as a valid event and is subsequently lost. This means using the lowest possible threshold value is crucial as smaller components of charge will be detected and included into the calculated total collected charge. 


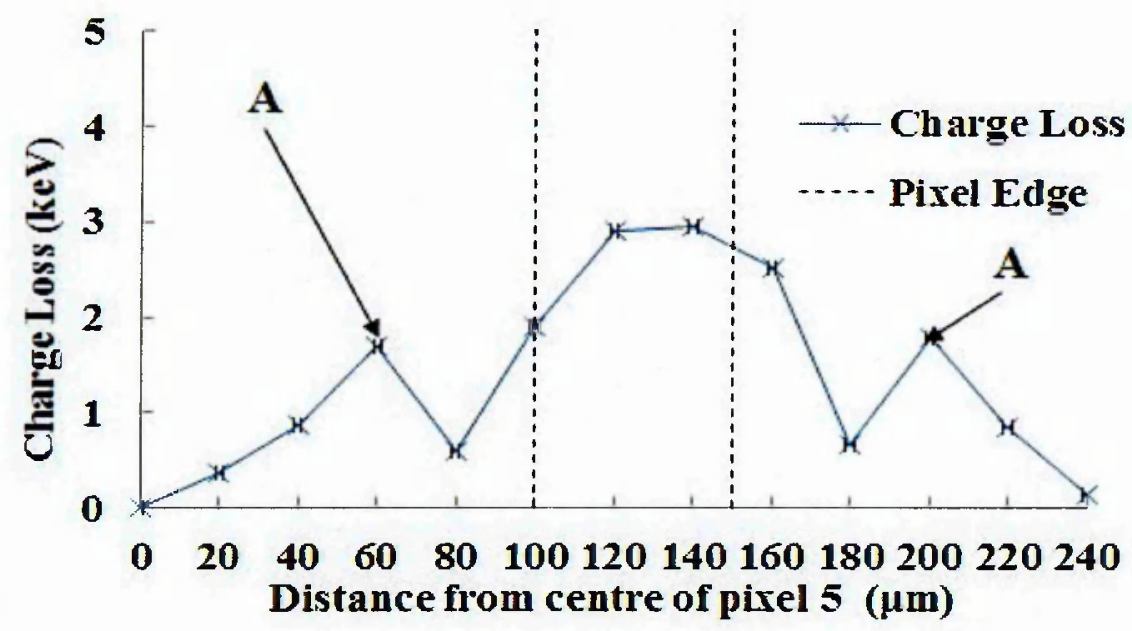

(a) $26 \mathrm{keV}$

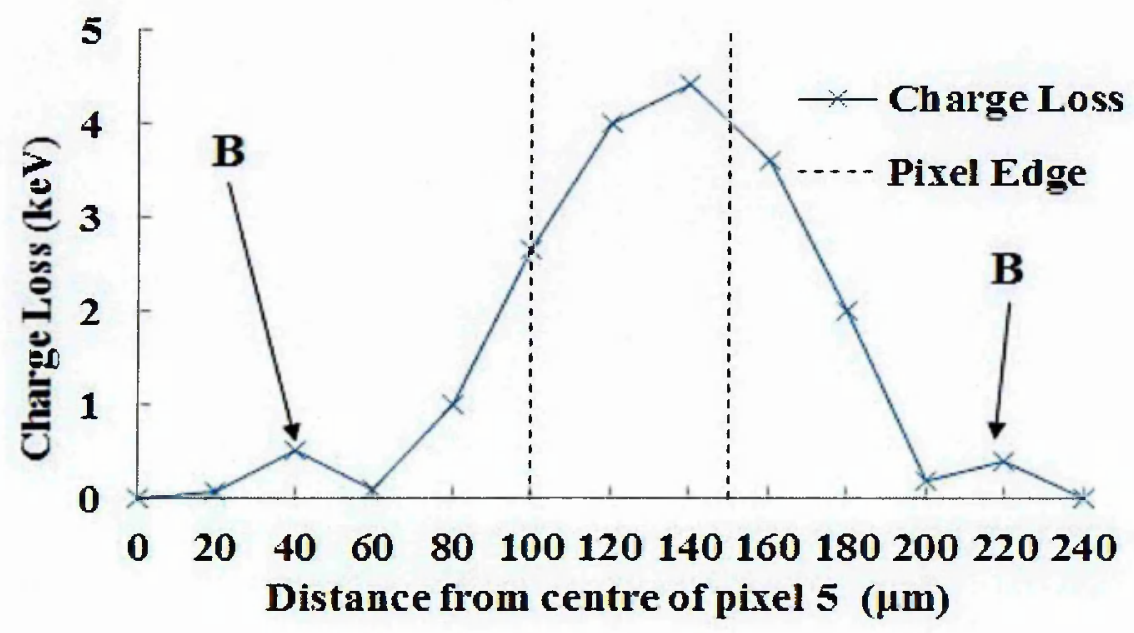

(b) $40 \mathrm{keV}$

Figure 5.10: Charge loss recorded on each position on the line scan between pixels 4 and 5. Points ' $A$ ' and ' $\mathrm{B}$ ' is due to a small compoinent of charge sharing is below the $1 \mathrm{keV}$ threshold is not record as a valid event and is subsequently lost. 


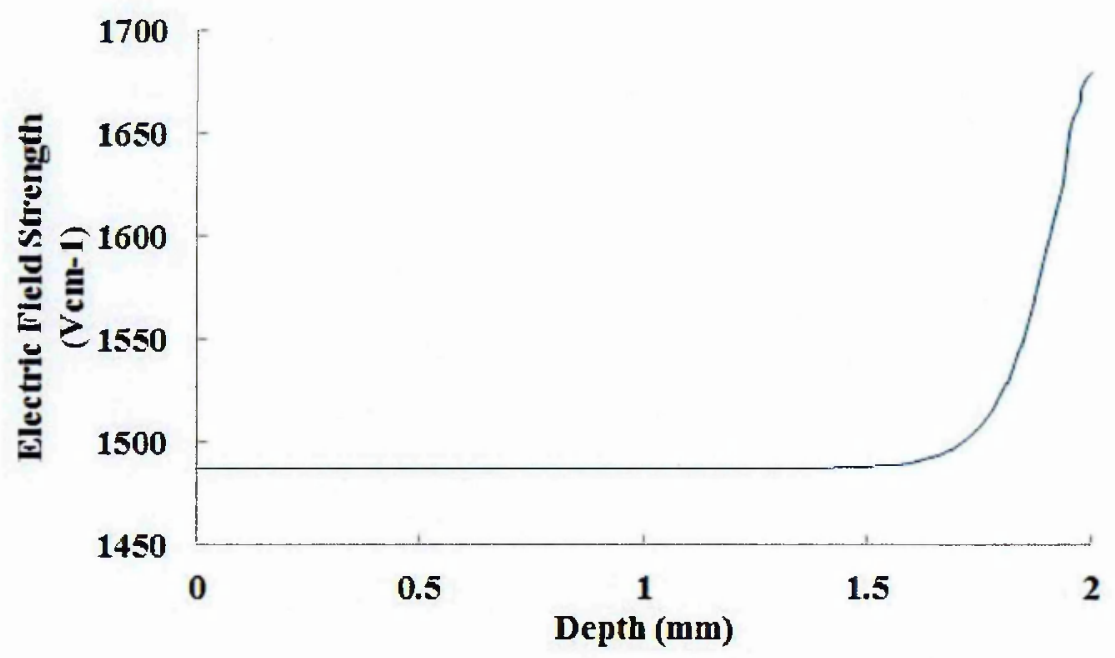

(a) Centre of a Pixel

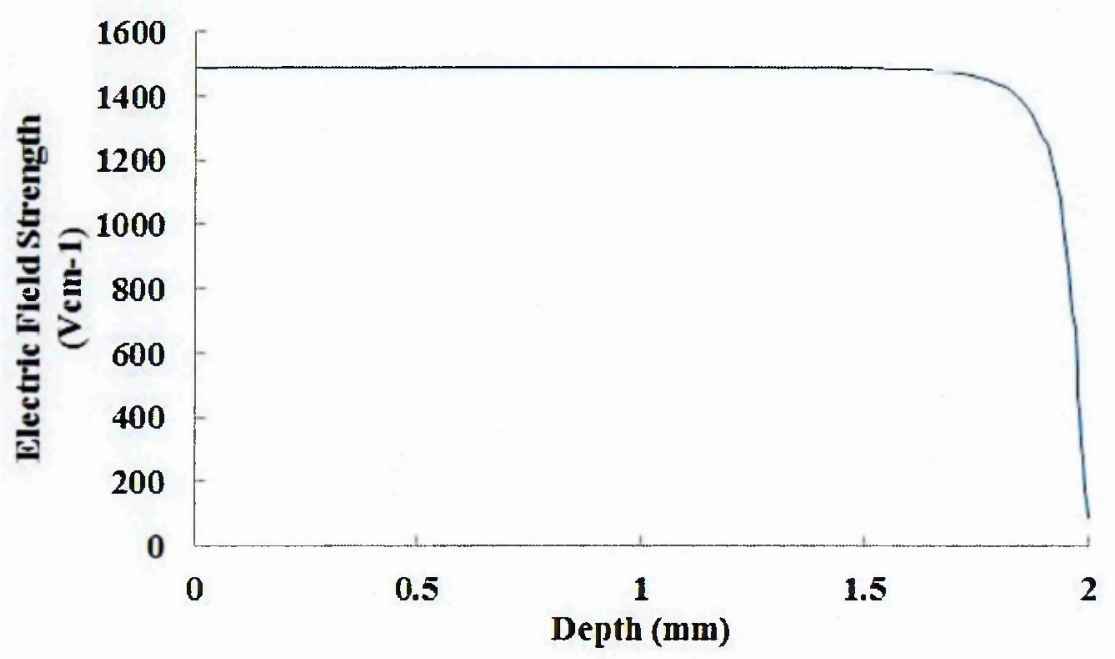

(b) Interpixel Region

Figure 5.11: A TCAD simulation of the electric field profile across the width of a PIXIE detector at a bias of $-300 \mathrm{~V}$. Where $0 \mathrm{~mm}$ is the cathode electrode and 2 $\mathrm{mm}$ is the anode electrode. Simulations for incident beamline positions in centre of a pixel and the interpixel region are shown to highlight the difference in field profiles [52]. 


\subsection{D Parametric Plot: Representation of Charge Sharing Events}

As previously explained it is possible to classify the interaction of an X-ray with a detector and the subsequent charge cloud which is created. On an event by event basis if the charge and subsequent equivalent energy collected by a pixel is greater than a user defined threshold $(1 \mathrm{keV})$ it is classified as a hit. The number of hits for each event is the multiplicity $(m)$ of an event. Therefore for a specific event if $m>1$ it is classified as a charge sharing event.

One way to represent data collected in $m=2$ charge sharing events is to create a $2 \mathrm{D}$ parametric plot $[56,57]$. A $2 \mathrm{D}$ plot is created by plotting the charge collected by pixel 4 (in $\mathrm{keV}$ ) against the charge collected by pixel 5 (in $\mathrm{keV}$ ) and the $Z$-axis value represents the number of events at the specific energy combination. It is possible to use these $2 \mathrm{D}$ parametric plots to investigate multiple events involving charge sharing in pixelated detectors. Therefore $2 \mathrm{D}$ plots were created for the case when the 26 $\mathrm{keV}$ and $40 \mathrm{keV}$ beam were incident at positions of $100 \mu \mathrm{m}-160 \mu \mathrm{m}$ from the centre of pixel 5. At a detector bias of $-300 \mathrm{~V}$ all the events are found to be classified as $m$ $=2$ events and is subsequently possible to create a $2 \mathrm{D}$ plot.

\subsubsection{D plots Recorded with a $26 \mathrm{keV}$ Beam}

Figure 5.12 shows the $2 \mathrm{D}$ plots recorded with a $26 \mathrm{keV}$ beam incident in the interpixel region between pixels 4 and 5 of the $200 \mu \mathrm{m}$ pixel size and $250 \mu \mathrm{m}$ pitch array on the PIXIE detector. Each of the four 2D plots shown all have similar characteristics. Therefore to explain and analyse these effects the $2 \mathrm{D}$ plot and subsequent spectrum recorded at a beamline position of $140 \mu \mathrm{m}$ from the centre pixel 5 will be analysed in depth.

At each position on the line scan it is found that the majority of the events lie on a diagonal line like the red one shown in figure 5.13(a), which is known as the line of "equals sum energy". The line is known as this is if the energy recorded by pixel 4 and pixel 5 at each point on the line are summed together it will give the total 


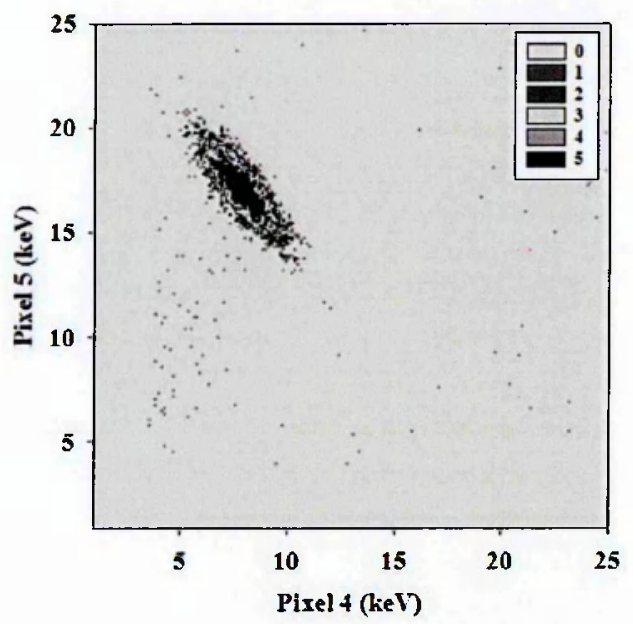

(a) $100 \mu \mathrm{m}$

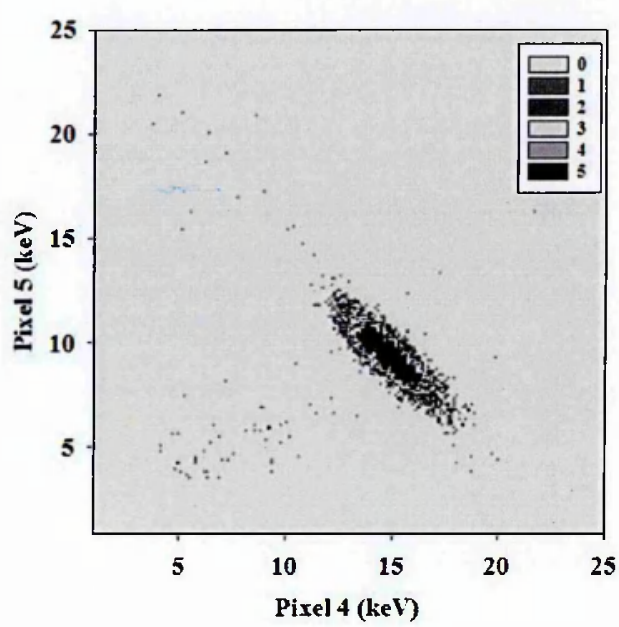

(c) $140 \mu \mathrm{m}$

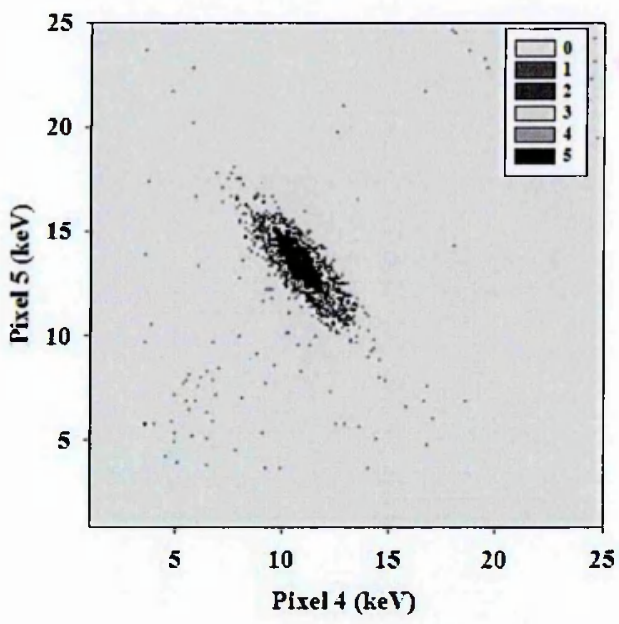

(b) $120 \mu \mathrm{m}$

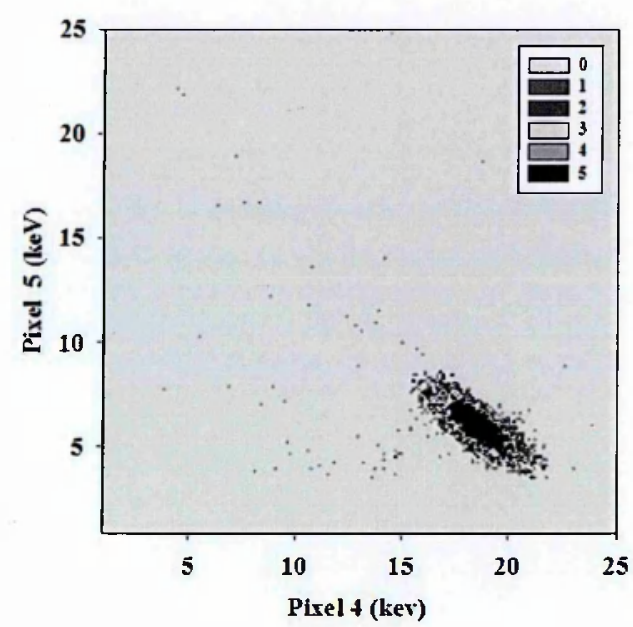

(d) $160 \mu \mathrm{m}$

Figure 5.12: 2D plots measured for the line scan between pixels 4 and 5 at beamline energy of $26 \mathrm{keV}$. The interaction positions are moving away from pixel 5 towards pixel 4 with interaction positions of $100 \mu \mathrm{m}-160 \mu \mathrm{m}$ from the centre of pixel 5 . 


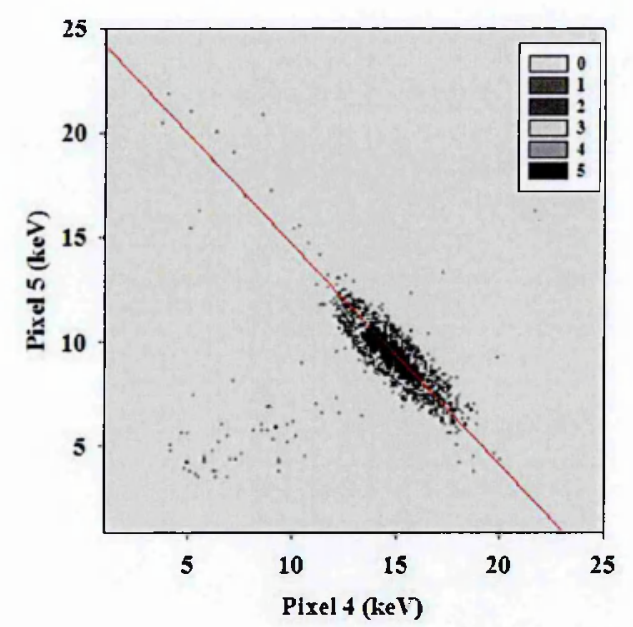

(a) 2D plot recorded at beam position of 140 $\mu \mathrm{in}$

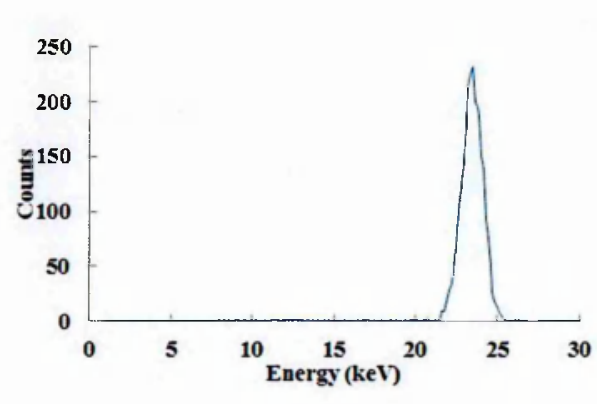

(b) Resulting spectrum recorded

Figure 5.13: The 2D plot and resulting spectrum recorded when the $26 \mathrm{keV}$ beam was incident at a position of $140 \mu \mathrm{m}$ from the centre of pixel 5 .

collected charge for that specific event. Furthermore if each event sum energy is subsequently plotted the resulting spectrum will be the one shown in figure 5.13(b). On each of the $2 \mathrm{D}$ plots the line of equals sum energy runs through a cluster of events. This cluster position is related to the amount of energy collected by each pixel at each position on the line scan. As previously shown in figure 5.8 at each beamline position the amount of charge collected by each pixel changes. The centre of the cluster relates to the peak centroid in $\mathrm{keV}$ of the single pixel spectrum recorded by the pixels of interest. For example the peak centroids of the spectra recorded by pixels 5 and 4 at a $26 \mathrm{keV}$ beam position of $140 \mu \mathrm{m}$ are $8.99 \mathrm{keV}$ and 14.44 $\mathrm{keV}$ respectively. Therefore the centre of the cluster shown on figure 5.13(a) is at a position of $14.44 \mathrm{keV}$ in the pixel 4 direction and $8.99 \mathrm{keV}$ in the pixel 5 direction. To investigate the properties of the location of the centre of a cluster the energy values for the centre of the cluster for each position on the line scan are plotted on the same graph. Figure 5.14 shows the peak centroid of the spectrum recorded by each of the two pixels shown on the $2 \mathrm{D}$ plots. The plot shows evidence of charge loss for beamline positions incident in the interpixel region as previously explained in section 5.4. If no charge loss was observed the points would lie on the black dashed 


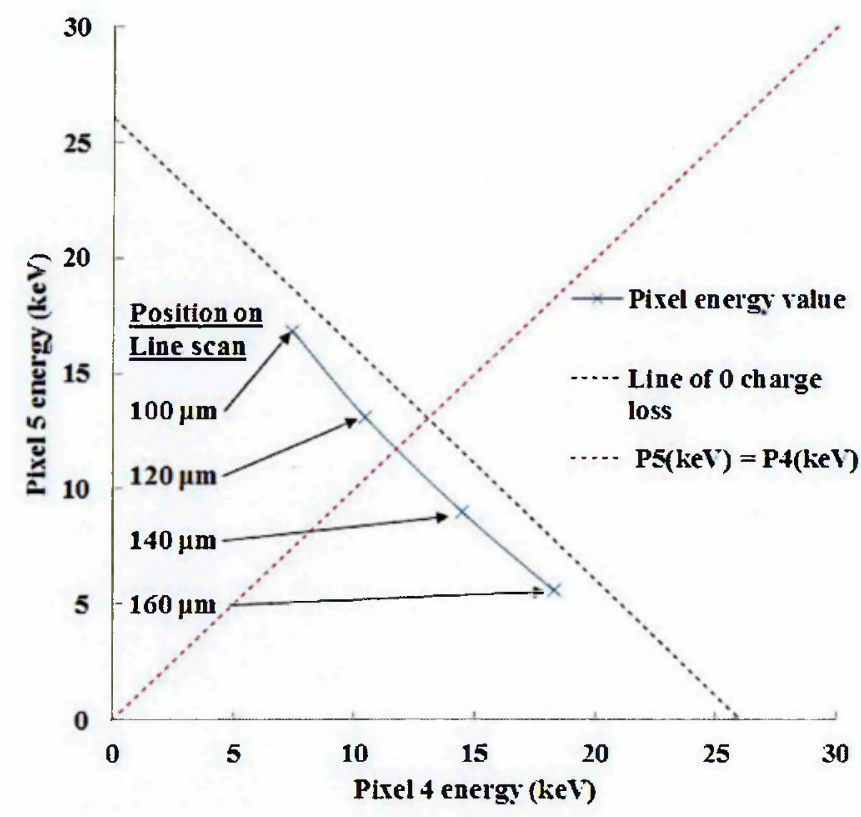

Figure 5.14: Plot of the recorded energy by each pixel relating the centre of the cluster shown on each of the $2 \mathrm{D}$ plots shown in figure 5.12 .

line shown in figure 5.14. All the points on this line relate to the case when the summed total collected charge is equal to the incident X-ray energy of $26 \mathrm{keV}$. The difference between the line and the summed energy found (blue points) is a measure of the charge loss.

The position of the centre of the cluster can also provide information on where beam line was incident in the interpixel region. Figure 5.14 shows a red dashed line which is the line of $P_{5}(\mathrm{keV})=P_{4}(\mathrm{keV})$. If a point was found to lie on this line the beam would be assumed to be in the centre of the interpixel region (125 $\mu \mathrm{m}$ in this case) as the charge has been equally shared between the two pixels. Therefore for energy co-ordinates found above the line the beam is nearer pixel 5 and for co-ordinates below it is nearer pixel 4 .

\subsubsection{D plots Recorded with a $40 \mathrm{keV}$ Beam}

2D plots were also produced for the case when the $40 \mathrm{keV}$ X-ray beam was incident on the same positions used previously for the $26 \mathrm{keV}$ beam. Figure 5.15 shows the 
resulting $2 \mathrm{D}$ plots which have certain differences compared to the ones generated with the $26 \mathrm{keV}$. To discuss these differences the $2 \mathrm{D}$ plot generated by the $40 \mathrm{keV}$ X-ray beam incident a position of $140 \mu \mathrm{m}$ from the centre pixel 5 will be analysed in greater detail.

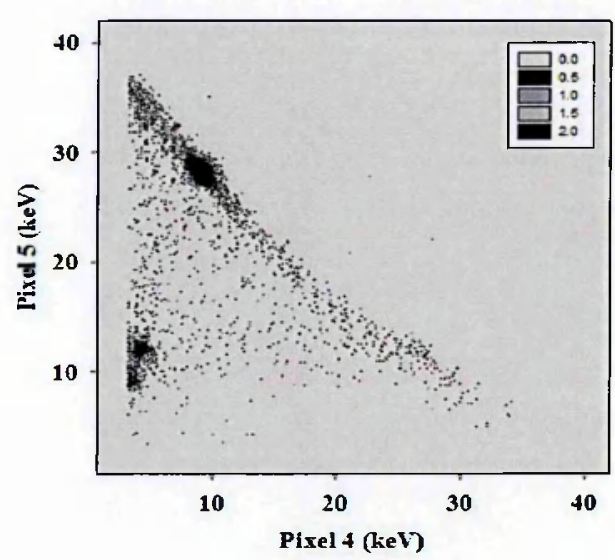

(a) $100 \mu \mathrm{m}$

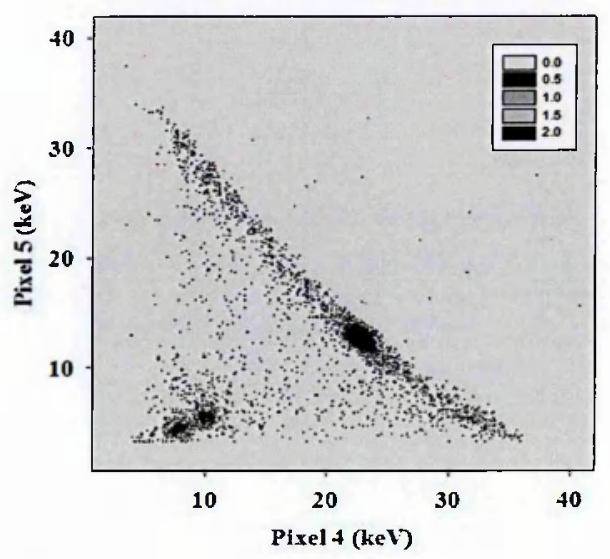

(c) $140 \mu \mathrm{m}$

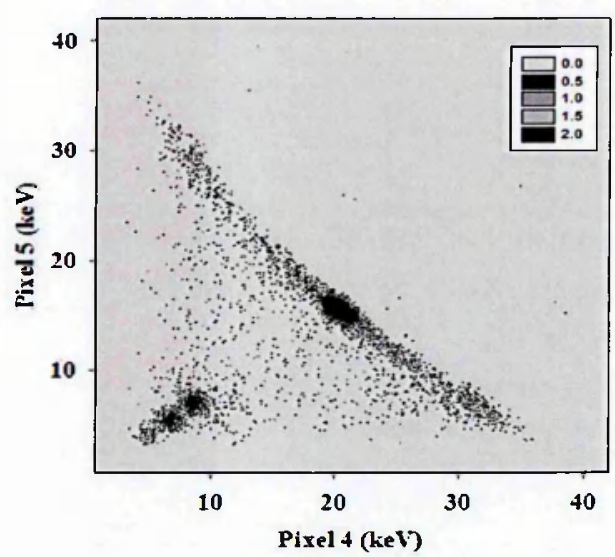

(b) $120 \mu \mathrm{m}$

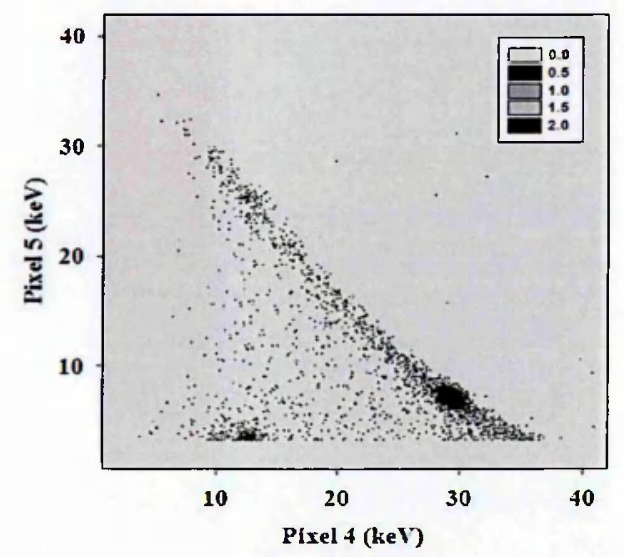

(d) $160 \mu \mathrm{m}$

Figure 5.15: 2D plots measured for the line scan between pixels 4 and 5 at beamline energy of $40 \mathrm{keV}$. The interaction positions are moving away from pixel 5 towards pixel 4 with interaction positions of $100 \mu \mathrm{m}-160 \mu \mathrm{m}$ from the centre of pixel 5 .

There are three distinct differences between the $2 \mathrm{D}$ plots generated at $40 \mathrm{keV}$ compared to the ones generated at $26 \mathrm{keV}$. To investigate this it is possible to gate on an energy region of interest in the LabVIEW analysis software and look at the 2D plot in generated in that energy region. 


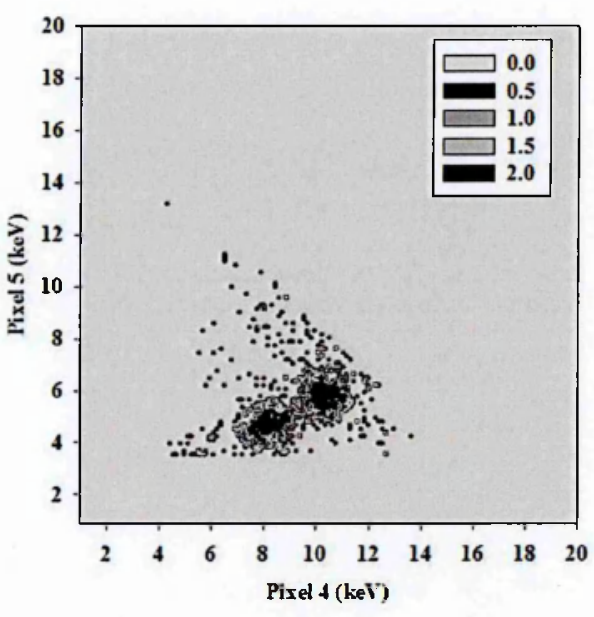

(a) $2 \mathrm{D}$ plot gated on the region relating to escape peaks

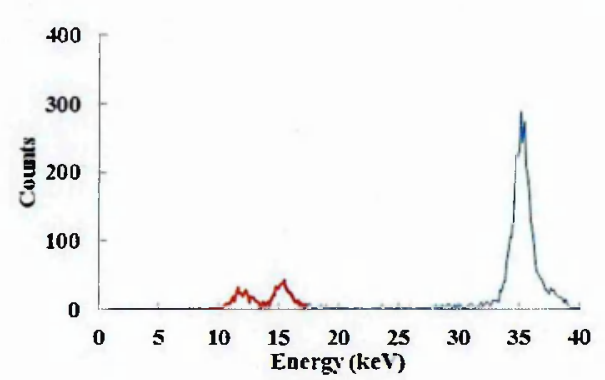

(b) Spectrum red line is region of interest

Figure 5.16: The 2D plot resulting from gating on the energy region relating to the creation of escape peaks in the sum spectrum shown.

Figure 5.16 shows the 2D plot generated when the software gates on the red region in the spectrum shown in the figure 5.16(b). This region is due to the creation and subsequent escape of $\mathrm{Cd} K_{\alpha}, \mathrm{Cd} K_{\beta}$ and $\mathrm{Te} K_{\alpha}$ fluorescence X-rays. It can be observed that the escape peak events are on a band of energies which is equal to the line of equal sum for the specific fluorescence X-ray. Furthermore there is a cluster of events which is the amount of charge collected by each pixel at this position on the line scan when a specific fluorescence X-ray escapes the detector.

The next region of interest is the region of the $2 \mathrm{D}$ plot between the bands relating to the escape peaks and the $40 \mathrm{keV}$ photopcak. As previously explained in section 5.2 the peak-to-valley ratio of the $40 \mathrm{kcV}$ photopeak is lower than the $26 \mathrm{keV}$ spectrum. For the case of the beam being incident at a position of $140 \mu \mathrm{m}$ from the centre of pixel 5 the peak-to-valley ratio is 50 at $26 \mathrm{keV}$ compared to 16 at $40 \mathrm{kcV}$. The decrease in the peak-to-valley ratio is not fully understood and should be investigated further at a later date.

The last area of interest on the 2D plot is the area relating to the photopeak. Figure 5.18(a) shows the region of the $2 \mathrm{D}$ plot which is due to the photopeak. As previously observed at $26 \mathrm{keV}$ the events recorded which generates the peak relating 


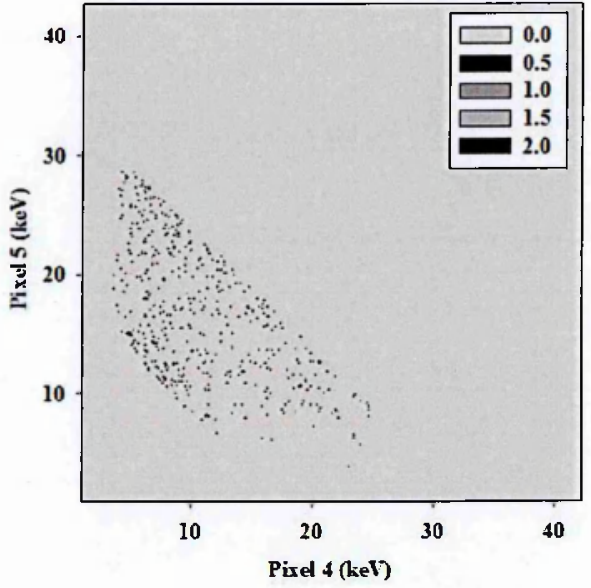

(a) 2D plot gated on the valley

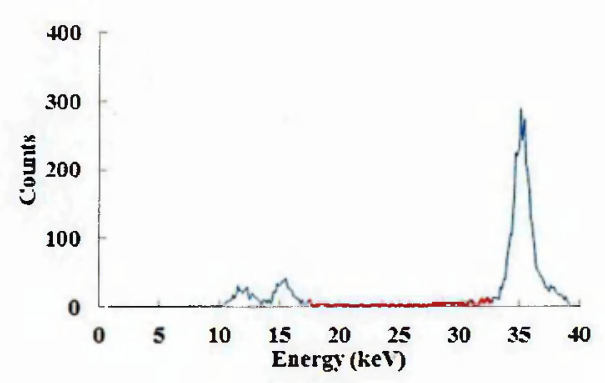

(b) Spectrum red line is region of interest

Figure 5.17: The 2D plot resulting from gating on the energy region relating to the creation of an increased valley region at X-ray beam energy of $40 \mathrm{keV}$.

to the total collected charge lie on a line of "equals sum energy" (red line on figures $5.18(\mathrm{a})-5.18(\mathrm{~b}))$. Thus it is possible to create a plot of the central cluster point for $40 \mathrm{keV}$ X-ray beam as previously done for $26 \mathrm{keV}$ as shown in figure 5.14. Figure 5.19 shows there is again evidence of charge loss in the interpixel region and the it is possible to classify where an event took place in the interpixel region by its position relating to the $P_{5}(\mathrm{keV})=P_{4}(\mathrm{keV})$ line.

There is however evidence that for certain events in this region there is less charge loss observed. As shown in figure 5.18(a) some events are above the red line which is equal to the line of "equals sum energy". Figure 5.18(d) shows the spectrum recorded at this position, the red counts on the spectrum is a high energy shoulder on the photopeak and result in the 2D plot shown in figure $5.18(\mathrm{c})$. The generation of these events with minimal charge loss is due to the creation and subsequent propagation of fluorescence X-rays. At energies greater than the $K$-shell absorption energy of $\mathrm{Cd}$ and $\mathrm{Te}$ it is possible for fluorescence X-rays to be emitted which can the travel up to $\sim 100 \mu \mathrm{m}$ in the detector (value calculated from [4]). Therefore it is possible for events in the interpixel region that the fluorescence X-ray will propagate through the detector and deposit its energy on a neighbouring pixel. This means 


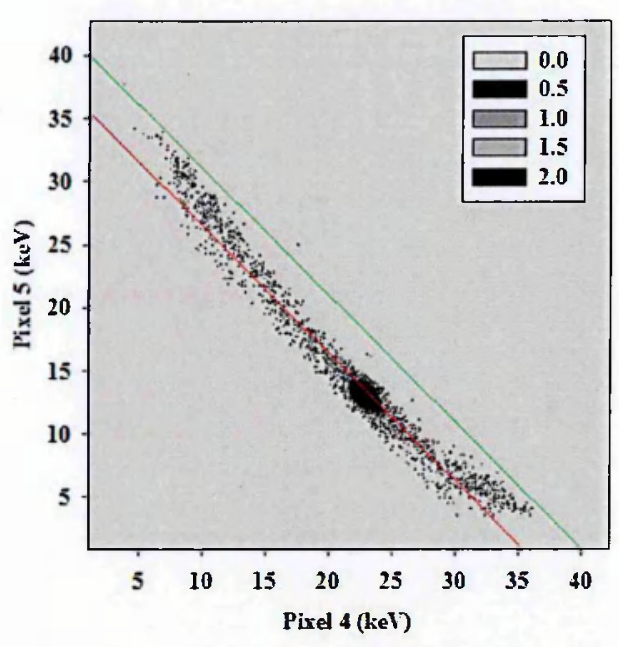

(a) $2 \mathrm{D}$ plot gate on the red and black region in figure (d)

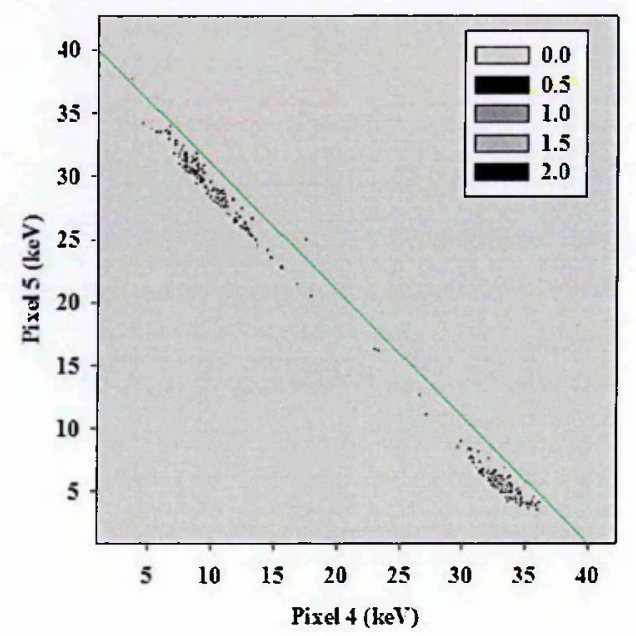

(c) 2D plot due to gating on high energy shoulder

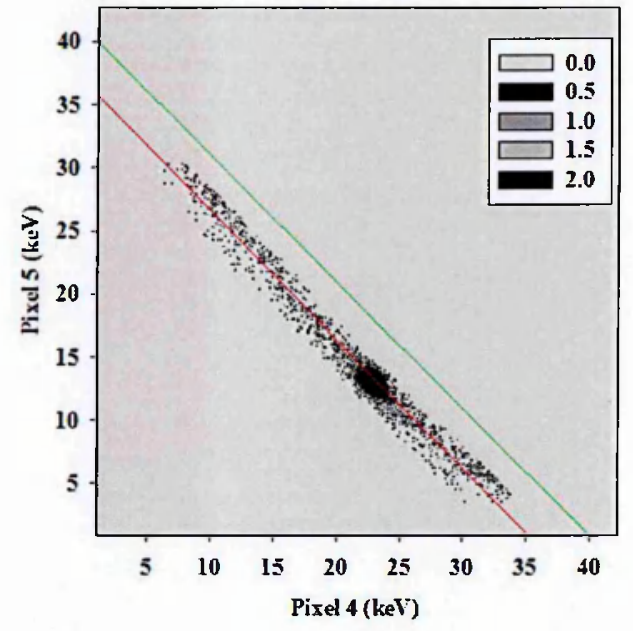

(b) $2 \mathrm{D}$ plot due gating on the black region in figure(d)

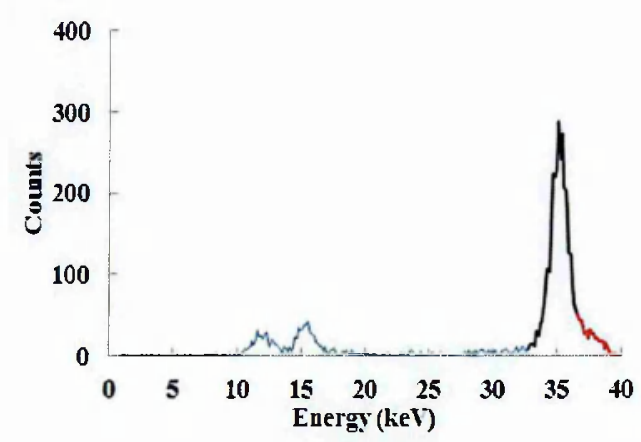

(d) recorded spectrum with regions of interest on.

Figure 5.18: 2D plots generated to analyse the band due to the recorded photopeak. 


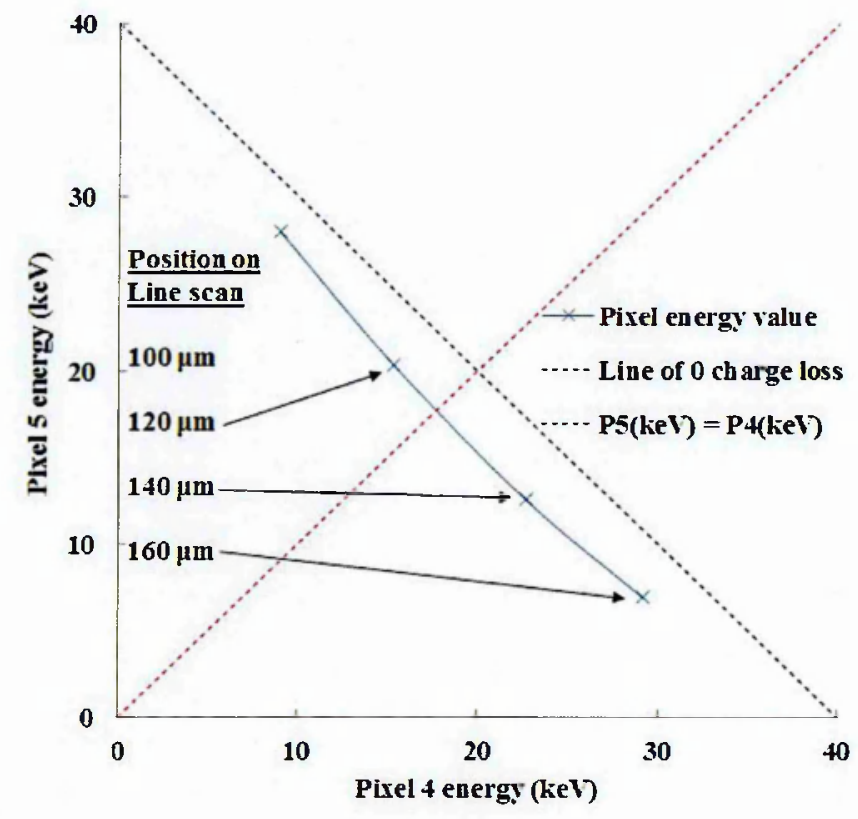

Figure 5.19: Plot of the recorded energy by each pixel relating the centre of the cluster shown on each of the $2 \mathrm{D}$ plots recording at a beamline energy of $40 \mathrm{keV}$ shown in figure 5.15.

that the amount charge in the cloud propagating towards the interpixel region will be smaller. Therefore less charge will be lost in the low electric field region resulting in the minimal charge loss observed in the $2 \mathrm{D}$ plot and the spectrum.

\subsection{Charge Sharing Between Adjacent Pixels at Different X-ray Energies}

To investigate charge sharing between pixels the number of single and charge sharing events was measured for each position on the line scan between pixels 4 and 5 at different incident X-ray energies with a detector bias of -300 V. Figure 5.20 shows the percentage of single $(m=1)$ and charge sharing $(m>1)$ events for each position on the line scan.

At a beamline encrgy of $26 \mathrm{keV}$ (figure $5.20(\mathrm{a})$ ) it is found that there are only charge sharing events in a region centred around the centre of the interpixel region. In this 


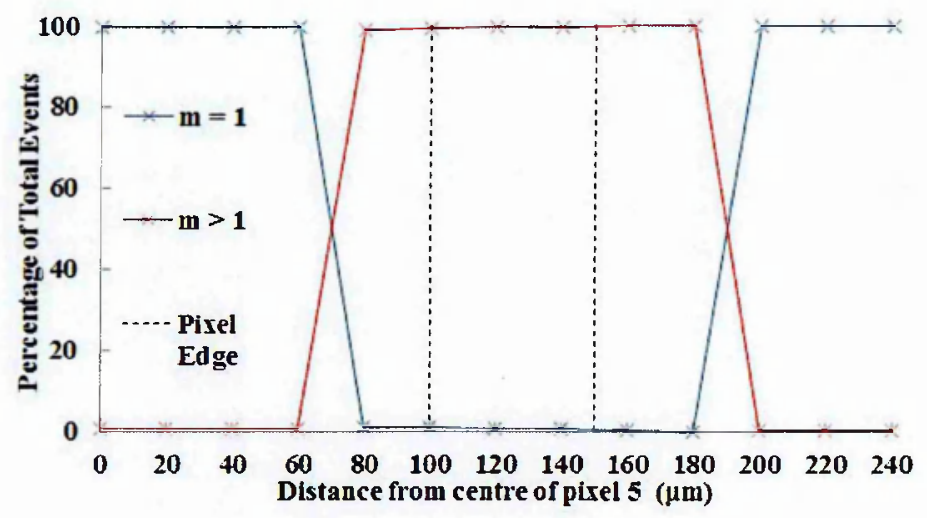

(a) $26 \mathrm{keV}$

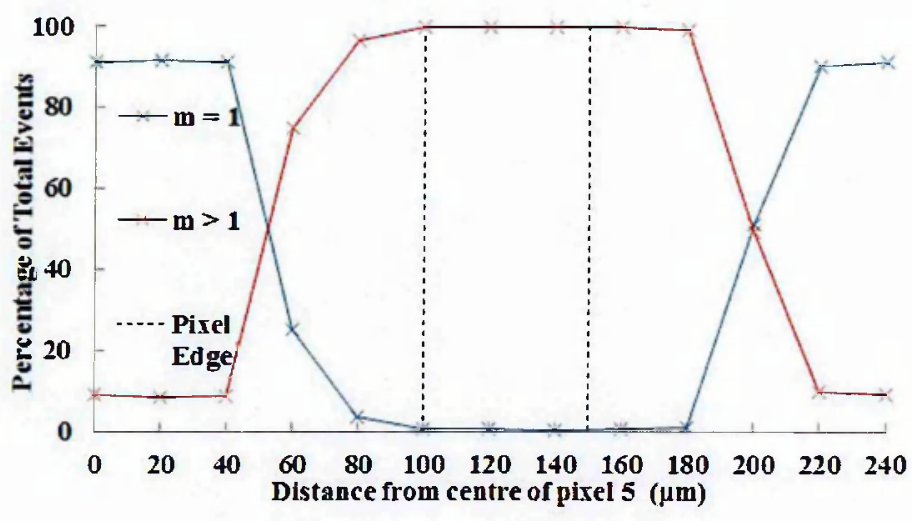

(b) $40 \mathrm{keV}$

Figure 5.20: Percentage of single (blue line) and charge sharing events (red line) for each position on the line scans between pixel 4 and 5 at a detector bias of $-300 \mathrm{~V}$ and X-ray energies of (a) $26 \mathrm{keV}$ and (b) $40 \mathrm{keV}$.

\begin{tabular}{|c|c|c|c|}
\hline Element & X-ray & Energy $(\mathrm{keV})$ & $\lambda_{\mathrm{CdZnTe}}(\mu \mathrm{m})$ \\
\hline \multirow{2}{*}{$\mathrm{Cd}$} & $K_{\alpha}$ & 23.18 & 116.7 \\
\cline { 2 - 4 } & $K_{\beta}$ & 26.10 & 160.6 \\
\hline \multirow{2}{*}{$\mathrm{Zn}$} & $K_{\alpha}$ & 8.63 & 8.7 \\
\cline { 2 - 4 } & $K_{\beta}$ & 9.57 & 11.4 \\
\hline \multirow{2}{*}{$\mathrm{Te}$} & $K_{\alpha}$ & 27.47 & 67.0 \\
\cline { 2 - 4 } & $K_{\beta}$ & 31.00 & 91.6 \\
\hline
\end{tabular}

Table 5.4: Attenuation lengths of $K$-shell fluorescence X-rays emitted from $\mathrm{Cd}, \mathrm{Zn}$ and $\mathrm{Te}$ in CdZnTe, (values calculated from [4]). 
region it found that $\sim 99 \%$ of the events have been charge shared between pixels 4 and 5 .

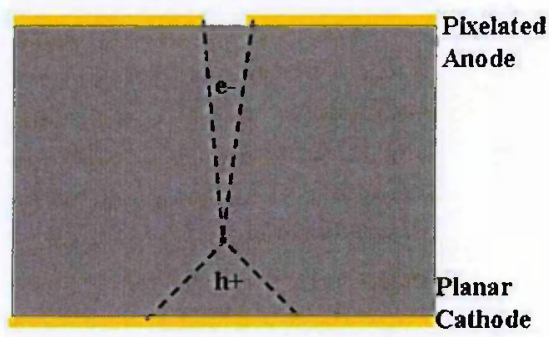

(a.) $\mathrm{E}<26.7 \mathrm{keV}$

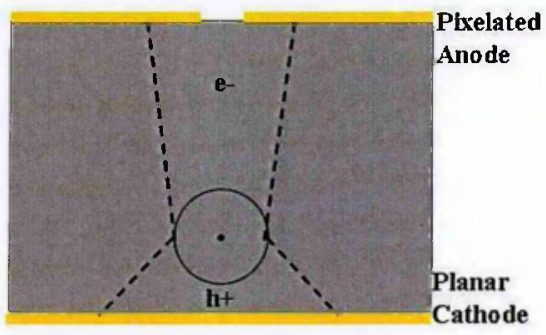

(b) $\mathrm{E} \geqslant 26.7 \mathrm{keV}$

Figure 5.21: Schematic representation of the charge cloud evolution in a CdZnTe $\mathrm{X}$-ray detector and the increase in the initial charge cloud size due to the creation of $K$-shell fluorescence X-rays.

In contrast, at $40 \mathrm{keV}$ (figure 5.20(b)) charge sharing events are found for all positions on the line scan even under the centre of a pixel. For some positions in the pixel boundaries it is found that $\sim 10 \%$ events are charge shared between pixels 4 and 5. Furthermore the width of the interpixel region where there is an increased level of charge sharing, is $140 \mu \mathrm{m}$ at $40 \mathrm{keV}$ compared to $100 \mu \mathrm{m}$ at $26 \mathrm{keV}$.

The reason for the increased charge sharing region and for charge sharing happening on every point of the line scan at $40 \mathrm{keV}$ is due to the propagation of fluorescence X-rays. Table 5.4 shows the attenuation length of $K_{\alpha}$ and $K_{\beta}$ fluorescence X-rays emitted by $\mathrm{Cd}, \mathrm{Zn}$, and Te in CdZnTe. For the case of the X-rays emitted by $\mathrm{Cd}$ and Te the attenuation length of the X-ray will effectively widen the initial charge cloud to sizes of $\sim 100 \mu \mathrm{m}$ as shown schematically in figure 5.21. The effect shows that the creation and subsequent propagation of fluorescence X-rays has a large effect on the detector response of a pixelated radiation detector.

\subsection{Detector Bias effect on Detector Characteris- tics}

As explained in section 2.6.2 when a X-ray interacts with a detector a cloud of electrons and holes are created which then propagate towards their respective attracting 
electrodes. During this propagation the charge cloud will diffuse resulting in a larger cloud compared to the initial size. It is possible to model the charge cloud diffusion with a Gaussian distribution. The charge cloud radius as a function of detector bias can be calculated as follows:

$$
r=1.15 \sqrt{\frac{2 k_{B} T d D}{q V}}
$$

where $r$ is the charge cloud radius, $d$ is the drift distance of the charge cloud, $D$ is the detector width, $k_{B}$ is Boltzmanns constant, $T$ is the absolute temperature, $q$ is the charge of the carriers and $V$ is the detector bias [41].

Accounting for the attenuation length of a $40 \mathrm{keV}$ X-ray in CdZnTe the charge cloud drift distance, $d$, in the Redlen CdZnTe PIXIE detector is $1.91 \mathrm{~mm}$ [15]. The resulting minimum charge cloud diameters, neglecting effects due to charge carrier repulsion, are $59 \mu \mathrm{m}$ and $102 \mu \mathrm{m}$ at $-300 \mathrm{~V}$ and $-100 \mathrm{~V}$, respectively. Line scans were carried out at $-100 \mathrm{~V}$ and $-300 \mathrm{~V}$ with a $40 \mathrm{keV} \mathrm{X}$-ray beam. At each point on the line scan the amount of charge sharing between pixels was measured to investigate how the amount of charge sharing varies with increased charge cloud size. Furthermore the amount of charge collected by each pixel and the total collected charge was measured to investigate the detector bias effect on the collection of charge.

It should be noted that the line scan performed at $-100 \mathrm{~V}$ is shorter than the previous line scans. The X-ray beam is incident at positions of $40 \mu \mathrm{m}-200 \mu \mathrm{m}$ from the centre of pixel 5. This is due to time constraints at the end of the experimental campaign.

\subsubsection{Charge Sharing at Different Detector Biases}

Figure 5.22 shows the multiplicity $(m)$ percentage for each position on the line scan between pixels 4 and 5 . Over the majority of the $-100 \mathrm{~V}$ scan it is found that $100 \%$ of the events are charge sharing events. At the ends of the line scan the percentage of $m \geqslant 1$ events decreases but is still dominating over the $m=1$ events. These results are different compared to $-300 \mathrm{~V}$ line scan. The region where the majority 
of events of charge sharing is smaller for the $-300 \mathrm{~V}$ line scan compared to the -100 $\mathrm{V}$ line scan. This increase in charge sharing at lower detector biases is due to the longer charge cloud drifting time thus the final charge cloud diameter is larger due to increased diffusion.

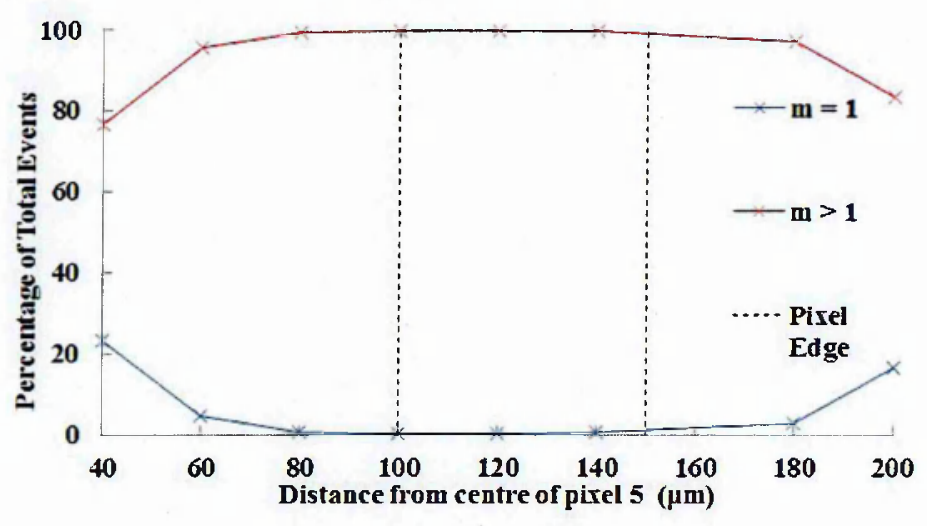

(a) $\mathrm{V}=-100 \mathrm{~V}$

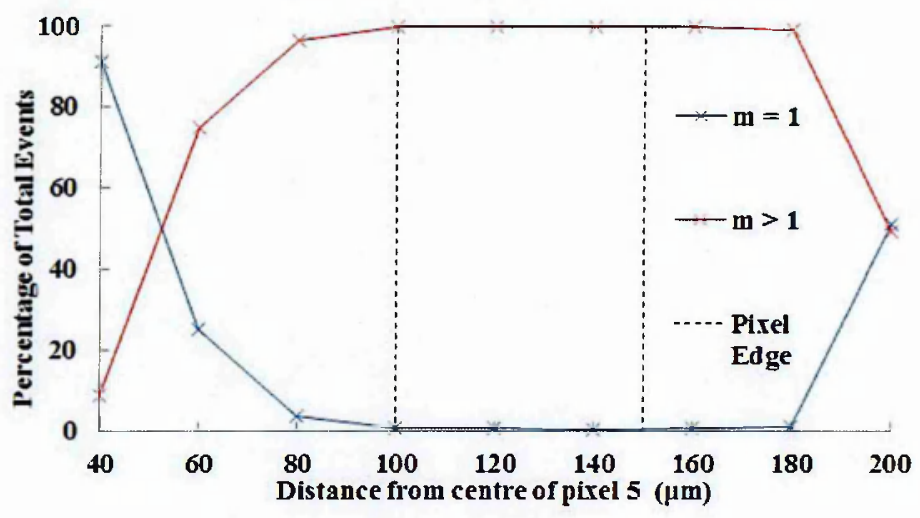

(b) $\mathrm{V}=-300 \mathrm{~V}$

Figure 5.22: Percentage of single (blue line) and charge sharing events (red line) for each position on the line scans between pixel 4 and 5 with X-ray beam energy of 40 $\mathrm{keV}$ and detector biases of (a) $-100 \mathrm{~V}$ and (b) $-300 \mathrm{~V}$.

\subsubsection{Variation in Total Collected Charge at Different De- tector Biases}

Figure 5.23 shows how the peak centroid of the spectra collected by pixels 4,5 and the sum spectrum varies with interaction position for each detector bias. As shown 


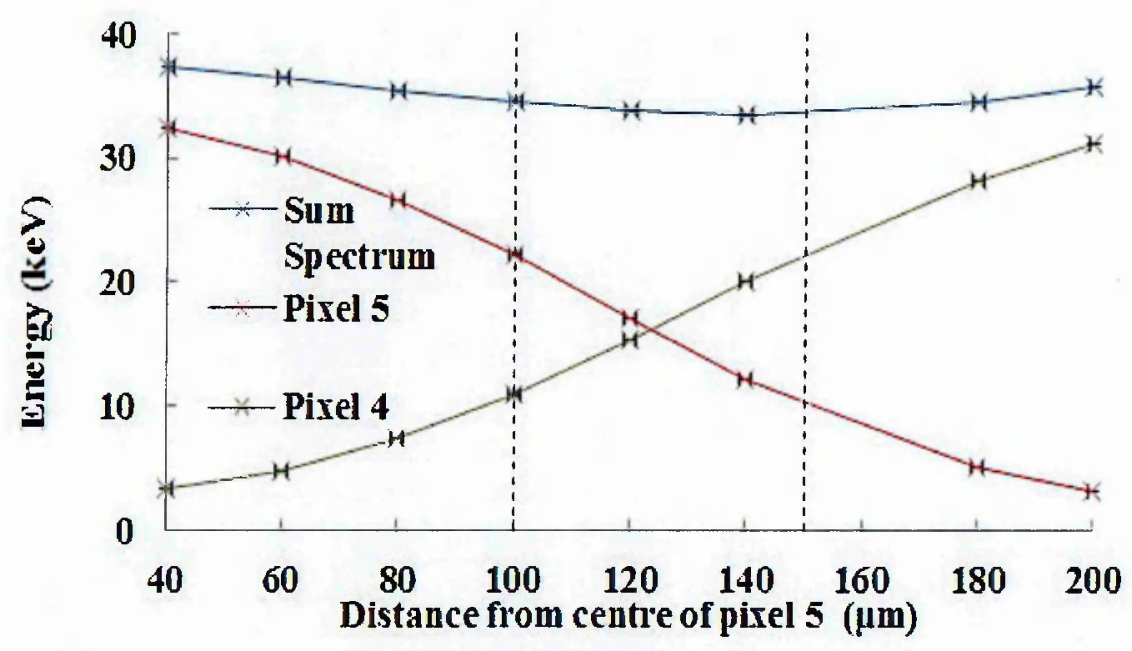

(a) $\mathrm{V}=-100 \mathrm{~V}$

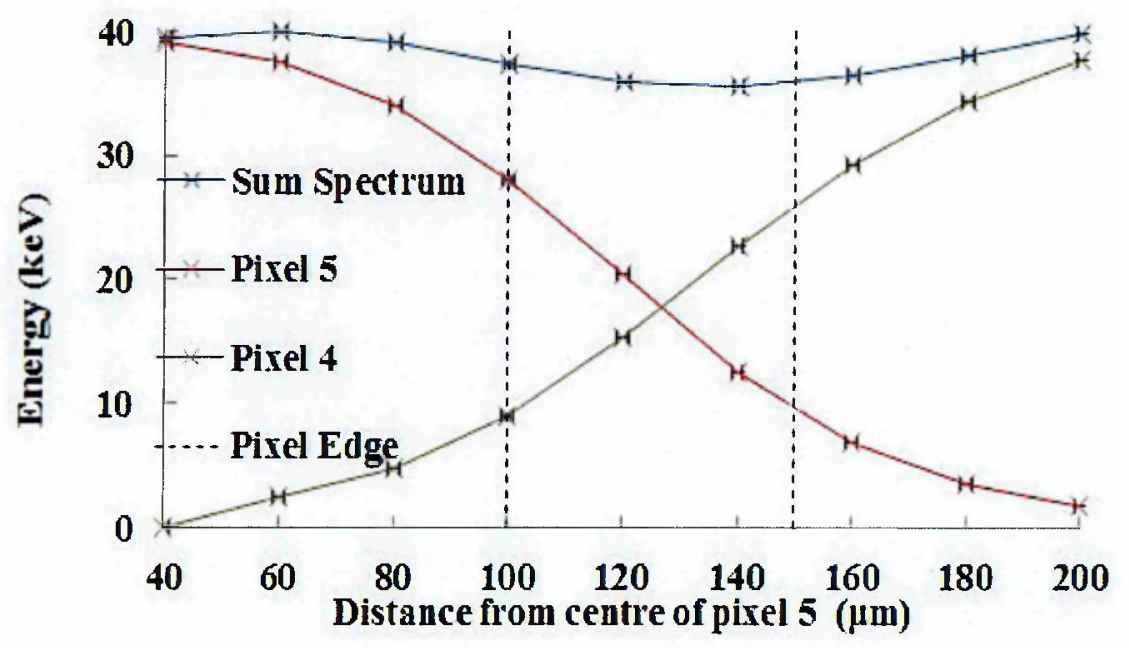

(b) $\mathrm{V}=-300 \mathrm{~V}$

Figure 5.23: The peak centroid energy of pixel 5, pixel 4 and the resulting sum spectrum at X-ray energy of $40 \mathrm{keV}$ and a detector bias of (a) $-100 \mathrm{~V}$ and (b) -300 V. 
in the figure for positions when the X-ray beam was incident in the interpixel region the amount of charge collected was less that the total generated charge.. Therefore level of charge loss at each position on the line scan is also calculated as shown in figure 5.24 .

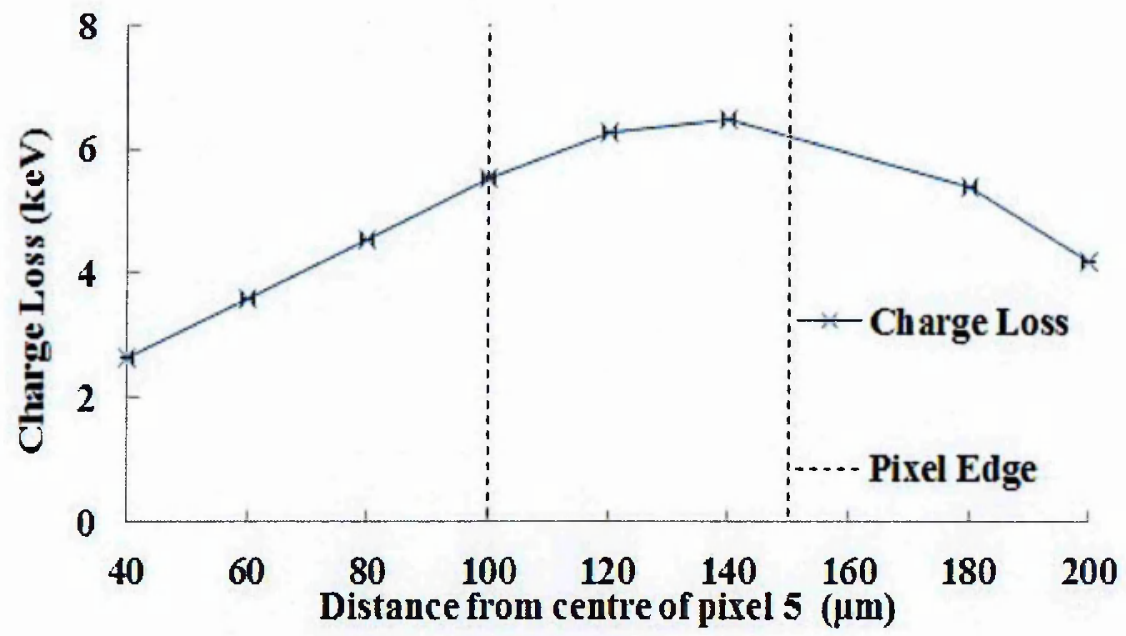

(a) $\mathrm{V}=-100 \mathrm{~V}$

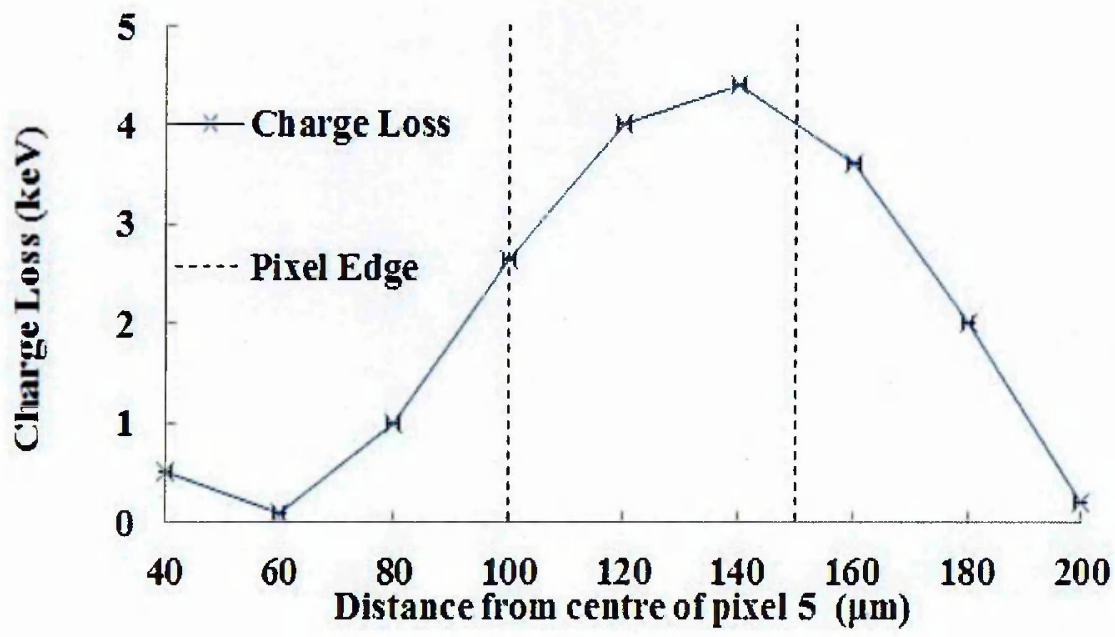

(b) $\mathrm{V}=-300 \mathrm{~V}$

Figure 5.24: Level of charge loss at each position on line scan between pixels 4 and 5 at different detector biases.

It is found that the level of charge loss increases at lower detector biases. For example at a beam line position incident $140 \mu \mathrm{m}$ from the centre of pixel 5, 6.48 $\mathrm{keV}$ of charge is lost at a detector bias of $-100 \mathrm{~V}$ compared to $4.41 \mathrm{keV}$ at $-300 \mathrm{~V}$. 
This equates to $5 \%$ increase in amount of charge being lost at $-100 \mathrm{~V}$. This increase in charge loss is due to the properties of the electric field at lower detector biases. As explained in section 5.4 the increased charge loss in the interpixel region is due to a local distortion in the electric field in this region resulting in a lower electric field strength. This in conjunction with a lower electric field strength across the device at lower detector biases will increases the amount of trapping of charge in the interpixel region. This as a result will increase the level of charge lost in the interpixel region at lower detector biases as observed in figure 5.24.

\subsection{Summary}

A large amount of data were taken on the 115 beamline at the Diamond Light Source. The beamline was collimated to a mean beam spot size of $20 \mu \mathrm{m}$ and line scans were undertaken between pixels 4 and 5 of array array 3 of the Redlen CdZnTe Pixie detector characterised in section 4.2. The primary area of interest for this experimental campaign was to investigate how the creation and subsequent propagation of fluorescence X-rays effects the detector response. Therefore energies below (26 keV) and above (40 keV) the $K$-shell fluorescence energy of Cd (26.7 keV) and $\mathrm{Te}(31.8 \mathrm{keV})$ were used.

At each position on the line scan the spectrum generated by each pixel was recorded. For example figure 5.4 shows the spectra collected by pixel 5 when the beamline was incident on the centre of that pixel. It was found that the FWHM of the spectra $(1.10 \mathrm{keV} @ 26 \mathrm{keV}$ and $1.15 \mathrm{keV} @ 40 \mathrm{keV})$ was very close to the ASIC generated noise of $1 \mathrm{keV}$. The major difference between the spectra recorded at $26 \mathrm{keV}$ and 40 $\mathrm{keV}$ was the evidence of $K_{\alpha}$ and $K_{\beta}$ escape peaks from $\mathrm{Cd}$ and Te. This resulted in a peak-to-valley ratio which was 5 times larger at $26 \mathrm{keV}$ compared to $40 \mathrm{keV}$.

In conjunction with single pixel spectra recorded at each position on the line scan the sum spectrum generated was also analysed. When the beamline was incident at regions in and around the interpixel region there was significant charge sharing between pixels. This resulted in a significant degradation of the spectroscopic 
performance, therefore a sum spectrum was generated (see figure 5.7). The peak centroid of the sum spectra and single pixel spectra were found to vary with interaction position of the beamline. To utilize this property a ratio $(\mathrm{R}(x))$ was defined see (equation 5.1 and figure 5.9) to calculate the interaction position of the beamline in the interpixel region. This ratio was found to be very accurate as the centre of the interpixel region (where $\mathrm{R}(x)=0$ ) was found to be a distance of $126 \mu \mathrm{m}$ from centre of pixel 5 when it is actually $125 \mu \mathrm{m}$ away from the centre.

Significant decrease in the total collected charge was also found when the beamline was incident in the interpixel region (see figure 5.10). It was found that at both beamline energies $\sim 11 \%$ of the created charge was not collected when the beam was incident in the centre of the interpixel region. The charge loss is thought to be due to a local distortion of the electric field in the interpixel region resulting in the electrons being trapped and subsequently not collected by a pixel.

2D parametric plots were generated for beam line positions in the interpixel region $(100 \mu \mathrm{m}-160 \mu \mathrm{m}$ from the centre of pixel 5). These parametric plots showed evidence of charge loss in the interpixel region at incident X-ray energies of $26 \mathrm{keV}$ and $40 \mathrm{keV}$. The creation and propagation of fluorescence X-rays had significant effects on the resulting 2D plots at X-ray energies of $40 \mathrm{keV}$. There are further bands relating to the line of "equals sum energy" for the creation and subsequent escape of $\mathrm{Cd}$ and $\mathrm{Te} K_{\alpha}$ and $K_{\beta}$ fluorescence X-rays. Furthermore the large propagation length of these fluorescence X-rays $(\sim 100 \mu \mathrm{m})$ resulted in events exhibiting less charge loss. This is due to the fluorescence X-rays propagating and depositing its created charge on a neighbouring pixel. This as a result meant less charge was propagating in the low electric field region in the interpixel region and subsequently less charge is trapped and not collected.

At each position on the line scan the level of charge sharing between pixels was recorded. At an incident X-ray energy of $26 \mathrm{keV}$ it was found that charge sharing only happened in a $100 \mu \mathrm{m}$ region centred on the centre of the interpixel region. By comparison at $40 \mathrm{keV}$ charge sharing was found to happen at every position on the line scan. For positions near the centre of a pixel $\sim 10 \%$ of the events were charge 
shared. Furthermore the region where increased charge sharing occurred was larger at $140 \mu \mathrm{m}$ region centred on the centre of the interpixel region.

The effect the detector bias had on the level of charge sharing between pixels and the charge loss in the interpixel region was also investigated. At a beam energy of $40 \mathrm{keV}$ charge sharing events were found to dominate at every position around the interpixel region at detector bias of $-100 \mathrm{~V}$ compared to a region of $140 \mu \mathrm{m}$ at $-300 \mathrm{~V}$ (see figure 5.22). The increase level of charge sharing is due to the longer drifting times at lower detector biases resulting in a more diffuse larger charge cloud. The level of charge loss in the interpixel region was also found to increase at lower detector biases. This is due to the decrease in the bulk electric field at lower detector biases resulting in increased trapping in the lower electric field region in the interpixel. These effects result in more charge getting trapped thus an decrease in the total collected charge. 


\section{Chapter 6}

\section{Conclusions and Future Work}

\subsection{Conclusions}

In recent years a great amount of scientific research has been carried out on $\mathrm{CdZnTe}$ as an attractive alternative to $\mathrm{Si}$ and $\mathrm{Ge}$ as a semiconductor radiation detector. It's reasonably good electron charge transport properties and larger band gap means $\mathrm{CdZnTe}$ can be used in a variety of applications. The main limiting factor of $\mathrm{CdZnTe}$ is it's poor hole transport properties. The mobility lifetime product for holes $(\mu \tau)_{h}$ is typically 2 orders of magnitude smaller compared to the electrons. The poor hole transport properties result in a drop in spectroscopic performance of the CdZnTe material. As a result of this much research has been undertaken on modifying the anode electrode so the resulting signal generated by the creation of electron-hole pairs in the CdZnTe is dominated by the electron motion. One example of single charge carrier sensitive device is one with an anode with an array of small pixels. The modified weighting potential results in the electron component dominating, furthermore there are multiple interesting effects such as charge sharing and charge loss between adjacent pixels. Therefore the PIXIE ASIC was developed to investigate these properties and was used in this work.

Initially a piece of LabVIEW software was written to analyse the data taken from a $2 \mathrm{~mm}$ thick Redlen CdZnTe detector. The parameters which operated the CR$(\mathrm{RC})^{n}$ digital shaper had to be optimized to get the best recorded spectroscopic 
performance of the detector. During the optimization of the time constant of the digital CR-(RC) ${ }^{n}$ shaper it was found the spectroscopic performance of a ${ }^{241} \mathrm{Am}$ spectrum collected by a single pixel went through a minima at a value of $0.5 \mu \mathrm{s}$. The properties of the FWHM trend is due to how different components of electronic noise contribute to the total recorded noise at different time constants. At values less than $0.5 \mu \mathrm{s}$ the series noise resulting from the input resistance of the PIXIE ASIC preamplifier for that pixel dominates. Furthermore at value greater than 0.5 $\mu$ s parallel noise dominates which is due to the leakage current of the device. The spectrum recorded by a single pixel was also used to measure how the detector bias effects the spectroscopic performance of a device. It was found that as detector bias increased the resulting FWHM of the $59.5 \mathrm{keV}{ }^{241} \mathrm{Am}$ peak improved. At higher detector bias the recorded leakage current increases which has a detrimental effect on the performance. However the charge collection efficiency improves at higher biases which dominates over the larger leakage current and therefore resulting in an improved spectroscopic performance.

The Redlen detector was also used to carry out investigations into effects involving multiple pixels. A gain matching procedure was carried out due to small discrepancies in the gain of pixel preamplifiers across an array. This results in a degradation of a sum spectrum calculated from the events recorded by the PIXIE detector. By use of a software threshold of $1 \mathrm{keV}$ it was possible to define an event by the number of pixels which had collected charge resulting in collected energy higher than $1 \mathrm{keV}$. The number of pixels per event is known as the multiplicity and the percentage of the total events for each multiplicity was recorded for a variety of spectra. It was found that as the pixel size increases the number of charge sharing events decreases. For example it was found that for a pixel array of pixel size of $350 \mu \mathrm{m}$ on a pitch of $500 \mu \mathrm{m}, 6 \%$ of the events were charge shared between pixels compared to $60 \%$ for a pixel array of $200 \mu \mathrm{m}$ pixels on a $250 \mu \mathrm{m}$ pitch. This is due to the resulting charge cloud from the interaction of a $59.5 \mathrm{keV}$ X-ray with the detector material has a final diameter of $60 \mu \mathrm{m}$ at a detector bias of $-300 \mathrm{~V}$ for the $2 \mathrm{~mm}$ thick detector. This is comparable to the pixel gap for the second array but is considerably 
smaller than the larger array resulting in less charge sharing. The level of charge sharing between pixels was measured for different incident X-ray energies, it was found as energy increased the level of charge sharing increased. Furthermore the increased level of charge sharing increased dramatically at energies above and below the $K$-shell absorption energy for $\mathrm{Cd}(26.7 \mathrm{keV})$ and Te $(31.8 \mathrm{keV})$. This was due to the creation and subsequent propagation of fluorescence X-ray. The propagation length of $\mathrm{Cd}$ and $\mathrm{Te} K_{\alpha}$ and $K_{\beta} \mathrm{X}$-rays range from $67 \mu \mathrm{m}$ to $160 \mu \mathrm{m}$. This initial propagation has the effect of increasing the initial size of the charge cloud resulting in larger final charge cloud. To investigate this effect further focused X-ray beam studies were carried out on the I15 beamline at the Diamond Light Source.

A focused X-ray beam was collimated to a mean spot size of $20 \mu \mathrm{m}$ and was moved in $20 \mu \mathrm{m}$ steps between the centre of pixel 5 (middle pixel) and the adjacent pixel, pixel 4. The incident $\mathrm{X}$-ray energies used were below (26 keV) and above (40 keV) the mean $K$-shell absorption energies of $\mathrm{Cd}$ and $\mathrm{Te}$. It was found that no charge sharing was found to occur between pixels for beam line positions near the centre of a pixel for the $26 \mathrm{keV} \mathrm{X}$-ray beam. In comparison charge sharing was found to happen at all beam line positions for X-ray energy of $40 \mathrm{keV}$. It was found that 10 $\%$ of events were charge shared for beam line positions near the centre of a pixel. Furthermore at $40 \mathrm{keV}$ the region where charge sharing dominated $(\mathrm{m} \geqslant 1=99 \%)$ was $140 \mu \mathrm{m}$ centred on the centre of interpixel region compared to $100 \mu \mathrm{m}$ at 26 $\mathrm{keV}$. Both of these effects are due to the creation and subsequent propagation of a fluorescence X-ray through the initial charge cloud. The propagation increased the initial charge could size resulting in increased level of charge sharing between pixels. For beamline positions incident in the interpixel region it was found that the total collected charge dropped. Peaking at $\sim 11 \%$ of charge lost this effect is due to a distortion of the electric field for positions in the interpixel region resulting in electrons being trapped and not collected.

The effect the detector biases has on charge sharing and charge loss in Redlen PIXIE pixelated radiation detectors was also investigated on the I15 beamline. A $40 \mathrm{keV}$ beam was again focused to $20 \mu$ and line scans were performed between 
adjacent pixels on the $200 \mu \mathrm{m}$ pixel size on a $250 \mu \mathrm{m}$ pitch pixel array. It was found that the region where charge sharing was dominant was significantly larger at $-100 \mathrm{~V}$ compared to $-300 \mathrm{~V}$. This is due to the increased final charge cloud size at a detector bias of $-100 \mathrm{~V}$ due to the decreased electric field and subsequent longer charge drifting times. The total collected charge was also measured at each position of the line scan. It was found that the maximum amount of charge loss happened when the $40 \mathrm{keV}$ beam was incident in the centre of the interpixel region. Furthermore the maximum charge loss was found to be $5 \%$ higher at a detector bias of $-100 \mathrm{~V}$. This is due to the decrease in the electric field across the device at lower detector biases resulting in an increased region of lower electric field strength in the interpixel region.

In future much research will be undertaken on CdZnTe Medipix detectors. These detectors have a significantly smaller pixel pitch $(55 \mu \mathrm{m})$ which makes it challenging to bond these detectors to an ASIC such as the Medipix II ASIC. Therefore a metrology study was carried out on a CdZnTe Medipix device to provide advice on its suitability to be Indium (In) bump bonded to a Medipix III ASIC. It was found that there was a large amount of curvature across the device with the height of device being 16-18 $\mu \mathrm{m}$ higher in the centre compared to the corners. This may result in the corner pixels being lost in the In bump bonding process as the height of the In bumps is $16-20 \mu \mathrm{m}$. Furthermore the characteristics of the fabricated gold pixels showed an increase in gold pixel height on two edges of the pixel. Through measurements with a Zygo non-contact optical profiler and a scanning electron microscope it was found the the pixel height increased by $154 \mathrm{~nm}$ on the edge of the pixel. This is thought to be due to an issue with the lift-off process in the gold pixel fabrication process.

\subsection{Future Work}

This work has shown that charge sharing and charge loss can have a significant effect on the recorded properties of a CdZnTe pixelated detector. The use of pixe- 
lated detectors in applications involving imaging result in the need for smaller and smaller pixel sizes. However as shown this results in an increase in charge sharing and a subsequent degradation in performance. Therefore significant work is being undertaken on CdZnTe detectors with smaller pixels sizes.

Initially work into using In bump bonding on detectors with smaller pixels is being undertaken, this will make it possible to bond CdZnTe with small pixels such as Medipix detectors where the pixels are on a $55 \mu \mathrm{m}$ pitch. Therefore metrology studies like the one carried out in this work will be undertaken to understand how successful the bonding procedure will be. The new Medipix III ASIC is of interest as it has a charge sharing correction facility making it possible be used in imaging applications whilst still accounting for the large amount of charge sharing between pixels. The final aim in this research is to evaluate the use of a $55 \mu \mathrm{m}$ pitch CdZnTe Medipix detector at the Diamond Light Source to investigate phenomena at smaller pixel pitches and its use in imaging applications. 
MATERIAL REDACTED AT REQUEST OF UNIVERSITY 


\section{List of Figures}

1.1 Probaility for photoelectric absorption to occur as function of energy for $\mathrm{Cd}_{0.9} \mathrm{Zn}_{0.1} \mathrm{Te}, \mathrm{Ge}$ and Si calculated using XCOM [4] . . . . . . . 4

2.1 Graph to show how the relative importance of the three major X-ray and $\gamma$-ray interactions vary with $Z$ of absorber and the energy of the interacting radiation $[3,19] \ldots \ldots \ldots \ldots$

2.2 Illustration of Compton scattering. A photon of energy $E_{\gamma}$ interacts with a 'nearly-free' electron. The photon scatters through an angle $\theta$ and the electron recoils through an angle $\Phi \ldots \ldots \ldots$. . . . . . 11

2.3 Mass attenuation coefficients for the three major electromagnetic radiation interactions with $\mathrm{Cd}_{0.9} \mathrm{Zn}_{0.1} \mathrm{Te}$, calculated using XCOM [4] . . 14

2.4 Schematic of electron orbitals of an atom and the resulting fluorescence X-rays emitted after the ejection of an electron via photoelectric

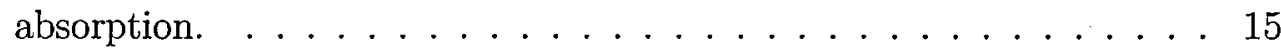

2.5 Mass attenuation coefficient of $\mathrm{Cd}_{0.9} \mathrm{Zn}_{0.1} \mathrm{Te}$ showing the discontinuities due to the binding engines of specific electron orbitals in $\mathrm{Cd}, \mathrm{Zn}$ and Te, calculated using XCOM [4] . . . . . . . . . . 15

2.6 Schematic representation of the charge cloud evolution in a CdZnTe $\mathrm{X}$-ray detector and the increase in the initial charge cloud size due to the creation of $K$-shell fluorescence X-rays. . . . . . . . . . . . 16

2.7 Spectrum collected by a CdZnTe detector show the escape peaks at energies less than the incident photon energy of $40 \mathrm{keV} \ldots \ldots$. . . . 17

2.8 The Zinc Blende structure of the compound semiconductor $\mathrm{ZnS}$ [22]. 18 
2.9 Band structure of direct and indirect band gap materials [23]. . . . . 19

2.10 The band gap of II/VI semiconductors as function of lattice constant

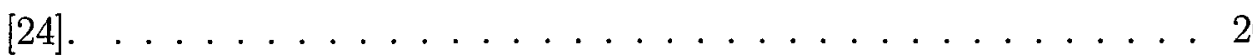

2.11 Electron-hole pair creation energy ( $W$-value) as a function of band gap for variety of different materials [24]. . . . . . . . . . . . . 21

2.12 (a) Schematic of a simple radiation detector with infinite planar cathodes and (b) its resulting weighting potential for the anode [12] . . . 24

2.13 The effect the rejection of slow rise time pulses has on the recorded spectroscopic performance of a ${ }^{137} \mathrm{Cs}$ spectrum taken by a $1.8 \times 1.8 \mathrm{x}$ $0.5 \mathrm{~cm} \mathrm{Cd} \mathrm{Cd}_{0.9} \mathrm{Zn}_{0.1}$ Te detector [31]. . . . . . . . . . . 25

2.14 (a) A schematic of a co-planar grid single charge carrier sensitive detector, (b) the weighting field for the three electrodes. The subtracted signal from electrodes 2 and 3 is predominantly due to electron drift close to the coplanar anodes $[37] . \ldots \ldots \ldots \ldots$

2.15 Schematic of drift strip detector. One detector cell is inbetween the dashed lines $\mathrm{A}$ and $\mathrm{B}$. The strips are biased so as to focus electrons onto the collecting anode [38].

2.16 (a) Schematic of a pixelated radiation detector and (b) its resulting weighting potential for signal read from pixel $1[3] . \ldots . . . . .28$

2.17 Weighting potential as read from a pixel for varying values of $\varepsilon / T$ showing the result of the 'small pixel effect' [3]. . . . . . . . . . . . 29

2.18 (a) Schematic of a pixelated radiation detector, (b) weighting potential of pixels which are adjacent to the main collecting pixel [3]. . . . 30

2.19 Schematics of the current pulses induced on the main collecting pixel and an adjacent pixel which results in zero net charge and a transient current pulse is induced on the collecting pixel.

2.20 Example of transient pulse being generated on a neighbouring pixel. A $78 \mathrm{keV}$ X-ray is collected by a pixel (blue pulse) and a transient pulse is subsequently induced on a neighbouring pixel (red pulse). . . 32 
3.1 Process flow for the creation of gold contact on a CdZnTe sample via the use of ma-N-410 negative photoresist [44] . . . . . . . . . . . 34

3.2 High resolution picture of Si sample with daisy chain test structure developed onto the sample before sputtering of gold contacts. . . . . . 35

3.3 Gold test structure after sputtering of gold contacts on Si sample. . . 35

3.4 Photo and schematic of the non-contact Zygo Newview 200 optical

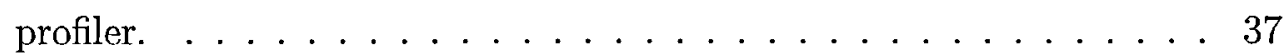

3.5 Picture of the PIXIE ASIC and the geometry of the pixel arrays for the PIXIE detectors used in this thesis. . . . . . . . . . . . 38

3.6 Block diagram of the individual pixel electronics. . . . . . . . . . 39

3.7 Photo of PIXIE ASIC readout system. . . . . . . . . . . . . 40

3.8 Photo of the XIA DGF-Pixie-4 system. . . . . . . . . . . . . . 41

3.9 Block diagram of the XIA DGF-Pixie-4 spectrometer [46]. . . . . . . 42

3.10 Pulses generated by the interaction of $60 \mathrm{keV}$ X-ray with pixel 5 of a Redlen CdZnTe detector. The pulses are recorded by the Pixie- 4 DAQ system and processed with the LabVIEW software. . . . . . . . 43

3.11 CR high pass filter or differentiator network and its effect on a step function input $[3] \ldots \ldots \ldots \ldots$. . . . . . . . . . . . . 44

$3.12 \mathrm{RC}$ high pass filter or differentiator network and its effect on a step function input $[3] \ldots \ldots \ldots \ldots \ldots \ldots \ldots$

3.13 Output of CR-(RC) ${ }^{n}$ shaper when applied to the pulse shapes shown in figure 3.10 .

3.14 Spectrum recorded by each pixel when a Redlen CdZnTe PIXIE detector was irradiated by a ${ }^{241} \mathrm{Am}$ source. Spectra in (b) have been calibrated using equation 3.6. It should be noted there is no recorded spectrum by pixel 1 as it is used to supply the bias to the guard ring of the detector. . . . . . . . . . . . . . . . . . 48

3.15 The multiplicity and hit pattern graphs generated by the LabVIEW software when array 4 of the Redlen PIXIE detector was irradiated by a ${ }^{241} \mathrm{Am}$ source. 
3.16 Sum spectrum generated by the LabVIEW software. Array 4 of the Redlen PIXIE detector was irradiated by a ${ }^{241} \mathrm{Am}$ source. . . . . . . 49

3.17 Photo of experimental setup used on the I15 beamline at the Diamond Light Source. . . . . . . . . . . . . . . . . . 51

3.18 Spectrum recorded by pixel 5 when the $26 \mathrm{keV}$ beam was incident on the centre of the pixel. Spectrum clearly shows the $26 \mathrm{keV}$ photopeak and the detection of $78 \mathrm{keV}$ and $104 \mathrm{keV}$ third and fourth harmonics of the beam. No second harmonic is ibserved due to the properties of the monochromator. . . . . . . . . . . . . . . 52

4.1 Macroscopic curvature of the CdZnTe Medipix detector. . . . . . . 55

4.2 Diagonal line scan through centre of sample. The red and blue profiles are top left to bottom right corner and top right to bottom left corner respectively. ......................... 56

4.3 Vertical line scan through centre of the sample. . . . . . . . . 56

4.4 Horizontal line scan through centre of the sample. . . . . . . . . 56

4.5 Profile of bare CdZnTe material with a calculated $R_{a}$ value of $6.56 \mathrm{~nm} .57$

4.6 Profile of gold pixel with a calculated $R_{a}$ value of $4.81 \mathrm{~nm}$. . . . . 58

4.7 Example pixels from Medipix detector showing increase in gold on right and bottom sides. . . . . . . . . . . . . . . . 59

4.8 Pixel and resulting pixel profile showing the pixel height and the feature on the right side of the pixel. . . . . . . . . . . 59

4.9 SEM image of two pixels to investigate gold profile on bottom and right edges. . . . . . . . . . . . . . . . 60

4.10 (a) SEM of the bottom right corner of figure 4.9 (b) to show increased height of Gold contact. (b) SEM of the top right corner of figure 4.9(b) to show comparison between raised and un-raised edge. . . . . 60

4.11 Single pixel ${ }^{241} \mathrm{Am}$ spectra recorded by arrays 3 and 4 at a detector bias of $-300 \mathrm{~V}$. It should be noted that no spectrum should be recorded by array 4 pixel 1 as it is used to supply bias to the guard ring of the detector. . . . . . . . . . . . . . . . . 62 
4.12 Single pixel ${ }^{241} \mathrm{Am}$ spectra recorded by array 4 pixel 7 at a detector bias of $-300 \mathrm{~V} \ldots \ldots \ldots \ldots$

4.13 The effect of the CR-(RC) ${ }^{4}$ shaper time constant on the FWHM of the $59.5 \mathrm{keV}^{241} \mathrm{Am}$ peak at a detector bias of $-300 \mathrm{~V}$. It should be noted the leakage current at $-300 \mathrm{~V}$ is $-0.84 \mathrm{nA}$. . . . . . . . . . 64

4.14 I-V characteristic of a single pixel to show how the leakage current increases with detector bias. . . . . . . . . . . . . . . 65

4.15 The effect of the CR-(RC) ${ }^{4}$ shaper time constant on the FWHM of the $59.5 \mathrm{keV}^{241} \mathrm{Am}$ peak at a detector bias of $-300 \mathrm{~V}$ and $-100 \mathrm{~V}$. The leakage current at each detector bias is also shown in the legend. 66

4.16 The effect number of steps of integration $(n)$ of the CR-(RC) ${ }^{n}$ shaper on the recorded detector performance. . . . . . . . . . 67

4.17 Resulting shaped pulses for different number of steps of integration (n) for the CR-(RC) ${ }^{n}$ shaper with a fixed time constant of $0.5 \mu \mathrm{s} . \quad .67$

4.18 The effect of detector bias on the single pixel performance of array 4 of the Redlen PIXIE detector. As previously explained no data is collected from pixel 1 as supplies bias to the guard ring of the detector. 69

4.19 Charge Collection Efficiency (CCE) and the resulting Hecht equation fits to calculate the $(\mu \tau)_{e}$ for each pixel of array $4 \ldots \ldots \ldots$

4.20 A TCAD simulation of the electric field profile across the width of a planar and PIXIE detector at a detector bias of $-300 \mathrm{~V}$. Where 0 $\mathrm{mm}$ is the cathode electrode and $2 \mathrm{~mm}$ is the centre of a pixel anode electrode [52]. . . . . . . . . . . . . . . . . . . 70

4.21 FWHM of a single pixel spectrum as function of the $(\mu \tau)_{e}$ value for that pixel. . . . . . . . . . . . . . 72

4.22 Uncalibrated single pixel spectra recorded by arrays 3 and 4 . To show the effect different gains and offsets have on the resulting peak centroid channel number of the $59.5 \mathrm{keV}$ characteristic X-ray. . . . . 73

4.23 Peak centroid of the $59.5 \mathrm{keV}$ peak as function of the $(\mu \tau)_{e}$ for each pixel of array $4 \ldots \ldots \ldots \ldots \ldots \ldots \ldots$ 
4.24 Spectrum produced by array 4 at a bias of $-300 \mathrm{~V}$ when the spectra recorded by each pixel are combined together. The two different 59.5 $\mathrm{keV}$ peaks labelled 'Peak A' and 'Peak B' are due to differences in the gains of pixels 3 and 7 compared to the remaining pixels. . . . . 75

4.25 Spectra recorded by pixels with different gains resulting in different peak centroid numbers and the non-gain matched sum spectrum shown in figure $4.24 \ldots \ldots$. . . . . . . . . . . . . . . 75

4.26 Pixel calibrations at $-300 \mathrm{~V}$ to calculate the gain matching parameters for arrays 3 and 4 of the PIXIE detector. . . . . . . . . . 76

4.27 Spectrum produced by array 4 at a bias of $-300 \mathrm{~V}$ when the spectra recorded by each pixel are gained matched and summed together. . . 77

4.28 The same data processed in two different ways to show effect of sum spectrum generation. Spectrum (a) shows only the energy from the single pixel with the highest energy in a $m \geqslant 2$ event. Spectrum (b) shows the sum of the recorded energy from all pixels above the user defined threshold of $3 \mathrm{keV}$ in each $m \geqslant 2$ event . . . . . . . . 78

4.29 FWHM of the $59.5 \mathrm{keV}$ peak from ${ }^{241} \mathrm{Am}$ at different threshold values for the $m \geqslant 1, m=1$ and $m=2$ sum spectra. . . . . . . . . . 79

4.30 Components of a charge sharing event inputted to sum spectrum at different threshold values. Showing at higher threshold values real charge sharing events are neglected. . . . . . . . . . . . 80

4.31 Multiplicity percentages recorded for array 3 and array 4 when irradiated with $59.5 \mathrm{keV}$ from ${ }^{241} \mathrm{Am}$ and a detector bias of $-300 \mathrm{~V}$. . . 81

4.32 Single pixel spectra recorded by pixel 7 from arrays 3 and 4 to show effect increased charge sharing has on spectroscopic performance.

4.33 Sum spectra recorded by array 3 and 4 to show effect increased charge sharing has on spectroscopic performance. . . . . . . . . . 82

4.34 The $\mathrm{m}=1$ and $\mathrm{m} \geqslant 2$ sum spectra recorded by array 3 at $-300 \mathrm{~V}$ to show the effect of charge sharing on spectroscopic performance. . . . 83 
4.35 Multiplicity percentages recorded for varying incident X-ray energies for array 3 at $-300 \mathrm{~V} \ldots \ldots \ldots . \ldots . \ldots . \ldots$

5.1 Counts/second recorded by pixel 5 on each point of scan to find centre of array 3 and 4 of the Redlen CdZnTe PIXIE detector. Colour code relates to the resulting pulse seen on the oscilloscope. . . . . . . 89

5.2 Orientation of the pixel numbers for array 3 of PIXIE detector as seen by the I15 beamline. . . . . . . . . . . . . . . . 90

5.3 The three line scans carried out to find the centre of pixel 5 of array 3 of the Redlen PIXIE detector. . . . . . . . . . . . . . . . . 91

5.4 Single pixel spectrum recorded by pixel 5 when the $\mathrm{X}$ ray beam was incident on the centre of the pixel. . . . . . . . . . . . . 93

5.5 Typical pulse shape and associated transient pulse induced on a neighbouring pixel. . . . . . . . . . . . . . . . . . . . . . 94

5.6 Single pixel spectrum recorded by pixel 5 for the X-ray beam incident on the centre the pixel with transient pulses removed and noise threshold of $1 \mathrm{keV} . \ldots \ldots \ldots \ldots \ldots$

5.7 (a) Separate spectra recorded by pixels 4 and 5, showing full energy peaks at $7.4 \mathrm{keV}$ and $16.8 \mathrm{keV}$ respectively. (b) sum spectrum from the same data showing the full peak at an energy of $24.2 \mathrm{keV}$. . . . 96

5.8 The peak centroid energy of pixel 5, pixel 4 and the resulting sum spectrum at a detector bias of $-300 \mathrm{~V}$ and X-ray energies below (26 $\mathrm{keV})$ and above $(40 \mathrm{keV})$ the $K$-shell absorption energies of Cd (26.7

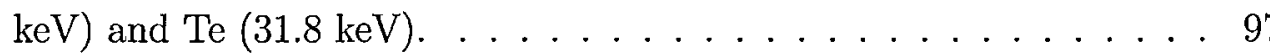

5.9 Linear relationship between the charge sharing ratio $\mathrm{R}(x)$ and the position of the X-ray beam in the interpixel region. . . . . . . . . . 98

5.10 Charge loss recorded on each position on the line scan between pixels 4 and 5. Points ' $A$ ' and ' $B$ ' is due to a small compoinent of charge sharing is below the $1 \mathrm{keV}$ threshold is not record as a valid event and is subsequently lost. . . . . . . . . . . . . . 99 
5.11 A TCAD simulation of the electric field profile across the width of a PIXIE detector at a bias of $-300 \mathrm{~V}$. Where $0 \mathrm{~mm}$ is the cathode electrode and $2 \mathrm{~mm}$ is the anode electrode. Simulations for incident beamline positions in centre of a pixel and the interpixel region are shown to highlight the difference in field profiles [52] . . . . . . . . 100

5.122 plots measured for the line scan between pixels 4 and 5 at beamline energy of $26 \mathrm{keV}$. The interaction positions are moving away from pixel 5 towards pixel 4 with interaction positions of $100 \mu \mathrm{m}-160 \mu \mathrm{m}$ from the centre of pixel $5 . \ldots \ldots$. . . . . . . . . . . 103

5.13 The $2 \mathrm{D}$ plot and resulting spectrum recorded when the $26 \mathrm{keV}$ beam was incident at a position of $140 \mu \mathrm{m}$ from the centre of pixel 5 . . . . 104

5.14 Plot of the recorded energy by each pixel relating the centre of the cluster shown on each of the $2 \mathrm{D}$ plots shown in figure 5.12 . . . . . . 104

$5.152 \mathrm{D}$ plots measured for the line scan between pixels 4 and 5 at beamline energy of $40 \mathrm{keV}$. The interaction positions are moving away from pixel 5 towards pixel 4 with interaction positions of $100 \mu \mathrm{m}-160 \mu \mathrm{m}$ from the centre of pixel $5 . \ldots \ldots \ldots$. . . . . . . . 106

5.16 The $2 \mathrm{D}$ plot resulting from gating on the energy region relating to the creation of escape peaks in the sum spectrum shown. . . . . . 107

5.17 The $2 \mathrm{D}$ plot resulting from gating on the energy region relating to the creation of an increased valley region at X-ray beam energy of 40 $\mathrm{keV} \ldots \ldots \ldots \ldots \ldots \ldots \ldots$

$5.182 \mathrm{D}$ plots generated to analyse the band due to the recorded photopeak.109

5.19 Plot of the recorded energy by each pixel relating the centre of the cluster shown on each of the $2 \mathrm{D}$ plots recording at a beamline energy

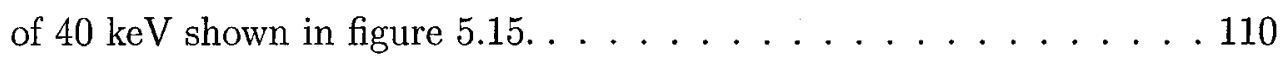

5.20 Percentage of single (blue line) and charge sharing events (red line) for each position on the line scans between pixel 4 and 5 at a detector bias of $-300 \mathrm{~V}$ and X-ray energies of (a) $26 \mathrm{keV}$ and (b) $40 \mathrm{keV}$. . . 111 
5.21 Schematic representation of the charge cloud evolution in a CdZnTe $\mathrm{X}$-ray detector and the increase in the initial charge cloud size due to the creation of $K$-shell fluorescence X-rays. . . . . . . . . . . 112

5.22 Percentage of single (blue line) and charge sharing events (red line) for each position on the line scans between pixel 4 and 5 with X-ray beam energy of $40 \mathrm{keV}$ and detector biases of (a) $-100 \mathrm{~V}$ and (b) -300 V. . . . . . . . . . . . . . . . . . . . . 114

5.23 The peak centroid energy of pixel 5, pixel 4 and the resulting sum spectrum at X-ray energy of $40 \mathrm{keV}$ and a detector bias of (a) -100

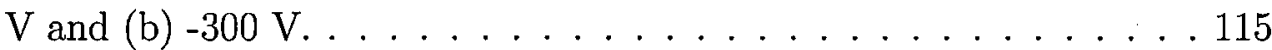

5.24 Level of charge loss at each position on line scan between pixels 4 and 5 at different detector biases. . . . . . . . . . . . . . 116 


\section{List of Tables}

1.1 Detector material properties of different semiconductor radiation de-

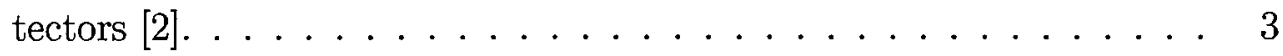

2.1 Attenuation lengths of $K$-shell fluorescence X-rays in $\mathrm{Si}$ and CdZnTe, (values calculated from $[4]) . \ldots \ldots \ldots \ldots$

2.2 Peaking time of the pulses shown in figure 2.20 showing that the transient pulse evolves and drops to zero over the time when the main pixel pulse is generated. . . . . . . . . . . . 32

3.1 The dimensions of the four different PIXIE arrays shown in figure 3.5. * Array 4 only has steering grid round four pixels. . . . . . . . . . 38

4.1 Height of curvature for the surface profiles shown in figures 4.2-4.4. . 55

4.2 The dimensions of the four different PIXIE arrays. . . . . . . . . . 61

4.3 The charge collection efficiency and leakage current measured at different detector biases. . . . . . . . . . . . . . . . 66 66

4.4 Signal-to-noise ratio for different values of $n \ldots \ldots \ldots 8$

4.5 Mobility Lifetime product $(\mu \tau)_{e}$ for each pixel of array 4 calculated from Hecht equation fits to data shown in figure $4.19 \ldots 71$

4.6 Peak centroid value for each pixel for the spectra shown in figure 4.22. 74

4.7 Gain and offsets for gain matching for arrays 3 and $4 \ldots \ldots 77$

4.8 FWHM of single and sum spectra shown in figures 4.32 and 4.33 respectively. . . . . . . . . . . . . . . . . 83

5.1 Details of the line scans to find the centre of pixel 5 of array 3. . . . 89 
5.2 Fluorescence X-ray energies and subsequent escape peaks shown in

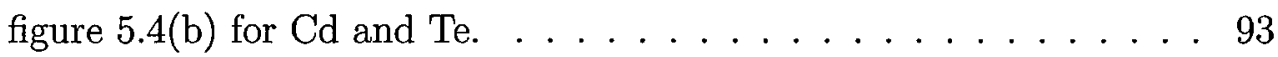

5.3 FWHM and Peak-to-Valley ratio of single pixel spectra recorded when the $26 \mathrm{keV}$ or $40 \mathrm{keV} \mathrm{X}$-ray beam was incident on the centre of pixel 5. 94

5.4 Attenuation lengths of $K$-shell fluorescence X-rays emitted from $\mathrm{Cd}$, Zn and Te in CdZnTe, (values calculated from [4]) . . . . . . . 111 


\section{Bibliography}

[1] A. Kastalsky, S. Luryi, and B. Spivak. Semiconductor high-energy radiation scintillation detector. Nucl. Instr. and Meth. A, 565(2):650-656, 2006.

[2] eV Products material properties data sheet. http://www . evmicroelectronics.com/pdf/material_prop.pdf, 2012.

[3] G.F. Knoll. Radiation detection and measurement. John Wiley and Sons, third edition, 1999.

[4] M.J. Berger, J.H. Hubbell, S.M. Seltzer, J. Chang, J.S. Coursey, R. Sukumar, D.S. Zucker, and K. Olsen. Xcom: Photon cross sections database. http: //www.nist.gov/pml/data/xcom/index.cfm, 2010.

[5] M. D. Wilson, R. Cernik, H. Chen, C. Hansson, K. Iniewski, L. L. Jones, P. Seller, and M. C. Veale. Small pixel CZT detector for hard X-ray spectroscopy. Nucl. Instr. and Meth. A, 652(1):158 - 161, 2011.

[6] P. Seller, S. Bell, R. J. Cernik, C. Christodoulou, C. K. Egan, J. A. Gaskin, S. Jacques, S. Pani, B. D. Ramsey, C. Reid, P. J. Sellin, J. W. Scuffham, R. D. Speller, M. D. Wilson, and M. C. Veale. Pixellated Cd(Zn)Te high-energy X-ray instrument. IOP. Journal of Instrumentation, 6(12):C12009, 2011.

[7] C. Christodoulou, C. B. Reid, D. O'Flynn, M. D. Wilson, M. C. Veale, R. J. Cernik, P. Seller, and R. D. Speller. Multivariate analysis of pixelated diffraction data. IOP. Journal of Instrumentation, 6(12):C12027, 2011. 
[8] M. C. Veale, J. Kalliopuska, H. Pohjonen, H. Andersson, S. Nenonen, P. Seller, and M. D. Wilson. Characterization of M- $\pi-n$ CdTe pixel detectors coupled to HEXITEC readout chip. IOP. Journal of Instrumentation, 7(1):C01035, 2012.

[9] E.N. Gimenez, R. Ballabriga, M. Campbell, I. Horswell, X. Llopart, J. Marchal, K.J.S. Sawhney, N. Tartoni, and D. Turecek. Characterization of Medipix3 With Synchrotron Radiation. IEEE Transactions on Nuclear Science, 58(1):323 $-332,2011$.

[10] T.E. Schlesinger, J.E. Toney, H. Yoon, E.Y. Lee, B.A. Brunett, L. Franks, and R.B. James. Cadmium zinc telluride and its use as a nuclear radiation detector material. Materials Science and Engineering: R: Reports, 32(4-5):103 - 189, 2001.

[11] H. H. Barrett, J. D. Eskin, and H. B. Barber. Charge transport in arrays of semiconductor gamma-ray detectors. Phys. Rev. Lett., 75:156-159, 1995.

[12] Z. He. Review of the Shockley-Ramo theorem and its application in semiconductor gamma-ray detectors. Nucl. Instr. and Meth. A, 463:250-267, 2001.

[13] M.D. Wilson, P. Seller, M.C. Veale, and P.J. Sellin. Investigation of the small pixel effect in CdZnTe detectors. IEEE Nuclear Science Symposium Conference Record, 2:1255-1259, 2007.

[14] C.M.H. Chen, S.E. Boggs, A.E. Bolotnikov, W.R. Cook, F.A. Harrison, and S.M. Schindler. Numerical modeling of charge sharing in cdznte pixel detectors. IEEE Transactions on Nuclear Science, 49(1):270 -276, 2002.

[15] M. Chmeissani, M. Maiorino, G. Blanchot, G. Pellegrini, J. Garcia, M. Lozano, R. Martinez, C. Puigdengoles, and M. Mullan. Charge sharing measurements of pixilated CdTe using Medipix-II chip. Proceedings of Instrumentation and Measurement Technology Conference, 1:787-791, 2004.

[16] G. Pellegrini, M. Chmeissani, M. Maiorino, G. Blanchot, J. Garcia, M. Lozano, R. Martinez, C. Puigdengoles, M. Ullan, and P. Casado. Performance limits of 
a $55 \mu \mathrm{m}$ pixel cdte detector. IEEE Transactions on Nuclear Science, $53(1): 361$ $-366,2006$.

[17] M.C. Veale, S.J. Bell, L.L. Jones, P. Seller, M.D. Wilson, C. Allwork, D. Kitou, P.J. Sellin, P. Veeramani, and R.C. Cernik. Investigating the Small Pixel Effect in CdZnTe Hard X-ray Detectors - The PIXIE ASIC. IEEE Nuclear Science Symposium Conference Record, pages 1255-1259, 2010.

[18] M.C. Veale, S.J. Bell, L.L. Jones, P. Seller, M.D. Wilson, C. Allwork, D. Kitou, P.J. Sellin, P. Veeramani, and R.C. Cernik. An ASIC for the Study of Charge Sharing Effects in Small Pixel CdZnTe X-Ray Detectors. IEEE Transactions on Nuclear Science, 58(5):2357 - 2362, 2011.

[19] R.D. Evans. The Atomic Nucleus. Krieger, 1982.

[20] M.J. Berger, J.S. Coursey, and J. Chang. ESTAR: Stopping-power and range tables for electrons. http://www.nist.gov/pml/data/xcom/index.cfm, 2005.

[21] M. O. Krause. Atomic radiative and radiationless yields for $K$ and $L$ shells. $J$. Phys. Chem. Ref. Data, 8:307-327, 1995.

[22] A. Barron and C. Smith. Crystal structure. http://cnx.org/content/ m16927/latest/, 2012.

[23] University of Cambridge. Direct and indirect band gap semiconductors. http: //www.doitpoms.ac.uk/tlplib/semiconductors/direct.php, 2012.

[24] A. Owens and A. Peacock. Compound semiconductor radiation detectors. Nucl. Instr. and Meth. A, 531:18-37, 2004.

[25] D. J. Olego, J. P. Faurie, S. Sivananthan, and P. M. Raccah. Optoelectronic properties of $\mathrm{Cd}_{1-x} \mathrm{Zn}_{x}$ Te films grown by molecular beam epitaxy on GaAs substrates. Appl. Phys. Lett., 47(11):1172 -1174, 1985.

[26] W. Shockley. Currents to conductors induced by a moving point charge. J. Appl. Phys., 9:635-636, 1938. 
[27] S. Ramo. Currents induced by electron motion. Proceedings of the IRE, $27(9): 584-585,1939$.

[28] G. Cavalleri, G. Fabri, E. Gatti, and V. Svelto. On the induced charge in semiconductor detectors. Nucl. Instr. and Meth., 21:177 - 178, 1963.

[29] M. Martini and G. Ottaviani. Ramo's theorem and the energy balance equations in evaluating the current pulse from semiconductor detectors. Nucl. Instr. and Meth., 67:177 - 178, 1969.

[30] G. Cavalleri, E. Gatti, G. Fabri, and V. Svelto. Extension of Ramo's theorem as applied to induced charge in semiconductor detectors. Nucl. Instr. and Meth., 92:137 - 140, 1971.

[31] J.C. Lund, R. Olsen, J.M. van Scyoc, and R.B. James. The use of pulse processing techniques to improve the performance of $\mathrm{Cd}_{1-x} \mathrm{Zn}_{x}$ Te gamma-ray spectrometers. IEEE Nuclear Science Symposium Conference Record, pages 126130, 1995.

[32] L.T. Jones and P.B. Woollam. Resolution improvement in CdTe gamma detectors using pulse-shape discrimination. Nucl. Instr. and Meth., 124(2):591 595, 1975.

[33] M. Richter and P. Siffert. High resolution gamma ray spectroscopy with CdTe detector systems. Nucl. Instr. and Meth. A, 322(3):529 - 537, 1992.

[34] H. Takahashi, D. Fukuda, T. Kurahashi, T. Iguchi, and M. Nakazawa. Signal processing for cdte detectors using a fast signal digitizing technique. Nucl. Instr. and Meth. A, 380(1-2):381-384, 1996.

[35] P.N. Luke. Single polarity charge sensing in ionization detectors using coplanar electrodes. Appl. Phys. Lett., 65(22):2884-2886, 1994.

[36] P.N. Luke. Unipolar charge sensing with coplanar electrodes-application to semiconductor detectors. IEEE Transactions on Nuclear Science, 42(4):207 $-213,1995$. 
[37] P.N. Luke and E.E. Eissler. Performance of CdZnTe coplanar-grid gamma-ray detectors. IEEE Transactions on Nuclear Science, 43(3):1481 -1486, 1996.

[38] I. Kuvvetli and C. Budtz-Jorgensen. Pixelated CdZnTe drift detectors. IEEE Transactions on Nuclear Science, 52(5):1975 - 1981, 2005.

[39] B.E Patt, J.S Iwanczyk, G Vilkelis, and Y.J Wang. New gamma-ray detector structures for electron only charge carrier collection utilizing high-Z compound semiconductors. Nucl. Instr. and Meth. A, 380(1-2):276-28, 1996.

[40] E Kalemci and J.L Matteson. Investigation of charge sharing among electrode strips for a CdZnTe detector. Nucl. Instr. and Meth. A, 478(3):527-537, 2002.

[41] K. Iniewski, H. Chen, G. Bindley, I. Kuvvetli, and C. Budtz-Jorgensen. Modeling charge-sharing effects in pixellated CZT detector. IEEE Nuclear Science Symposium Conference Record, 6:4608-4611, 2007.

[42] photolithogray explanation. http://en.wikipedia.org/wiki/ Photolithography, 2012.

[43] M.C. Veale. Charge Transport and Low Temperature Phenomena in Single Crystal CdZnTe. PhD thesis, University of Surrey, 2009.

[44] Microresist technology website. http://www.microresist.de/products/ negative_photoresists/ma_n_400_en.htm, 2012.

[45] DGF Pixie-4 - Multichannel Digital Gamma Processor. http://www.xia.com/ DGF_Pixie-4.html, 2012.

[46] W. Hennig, Y.X. Chu, H. Tan, A. Fallu-Labruyere, W.K. Warburton, and R. Grzywacz. The DGF Pixie-4 spectrometer - compact digital readout electronics for HPGe clover detectors. Nucl. Instr. and Meth. B, 263:175-178, 2007.

[47] Medipix homepage. http://medipix.web.cern.ch/medipix/, 2012. 
[48] C. Broennimann, F. Glaus, J. Gobrecht, S. Heising, M. Horisberger, R. Horisberger, H.C. Kstli, J. Lehmann, T. Rohe, and S. Streuli. Development of an Indium bump bond process for silicon pixel detectors at PSI. Nucl. Instr. and Meth. A, 565:303-308, 2006.

[49] P. Seller and M. Veale. Private Communication, 2012.

[50] P. Seller. Summary of thermal, shot and flicker noise in detectors and readout circuits. Nucl. Instr. and Meth. A, 426(2-3):538 - 543, 1999.

[51] M.C. Veale, P.J. Sellin, A. Lohstroh, A.W. Davies, J. Parkin, and P. Seller. X-ray spectroscopy and charge transport properties of CdZnTe grown by the vertical Bridgman method. Nucl. Instr. and Meth. A, 576:90-94, 2007.

[52] S. Bell and D. Duarte. TCAD Simulations of Planar and Pixelated Detectors. Private Communication, 2012.

[53] Synopsys Sentaurus TCAD: Technology Computer Aided Design. http://www . synopsys.com/Tools/TCAD/Pages/default.aspx, 2012.

[54] C. Allwork, D. Kitou, S. Chaudhuri, P. J. Sellin, P. Seller, M. C. Veale, N. Tartoni, and P. Veeramani. X-Ray Beam Studies of Charge Sharing in Small Pixel, Spectroscopic, CdZnTe Detectors. IEEE Transactions on Nuclear Science, 59(4):1563-1568, 2012.

[55] A.E. Bolotnikov, W.R. Cook, F.A. Harrison, A.-S. Wong, S.M. Schindler, and A.C. Eichelberger. Charge loss between contacts of CdZnTe pixel detectors. Nucl. Instr. and Meth. A, 430:326-331, 1999.

[56] I. Kuvvetli and C. Budtz-Jargensen. Measurements of charge sharing effects in pixilated CZT/CdTe detectors. IEEE Nuclear Science Symposium Conference Record, 3:2252 - 2257, 2007.

[57] A. E. Bolotnikov, S. E. Boggs, C. M. H. Chen, W. R. Cook III, F. A. Harrison, and S. M. Schindler. Optimal contact geometry for CdZnTe pixel detectors. Proc. SPIE, 4141:243-252, 2000. 